\title{
Transforming Oxadiazolines through Nitrene Intermediates by Energy Transfer Catalysis: Access to Sulfoximines and Benzimidazoles
}

\author{
Do Dam Park, ${ }^{a}$ Kwan Hong Min, ${ }^{a}$ Jihee Kang, ${ }^{a}$ Ho Seong Hwang, ${ }^{a}$ \\ Vineet Kumar Soni, ${ }^{a}$ Cheon-Gyu Cho, ${ }^{b}$ and Eun Jin Cho*a \\ ${ }^{a}$ Department of Chemistry, Chung-Ang University, Seoul 06974, Republic of Korea \\ ${ }^{b}$ Department of Chemistry, Hanyang University, Seoul 04763, Republic of Korea \\ E-mail: ejcho@cau.ac.kr
}

\section{Supporting Information}

$\begin{array}{ll}\text { General Considerations } & \text { S-2 }\end{array}$

$\begin{array}{ll}\text { Density Functional Theory Calculation Details } & \text { S-2 }\end{array}$

$\begin{array}{ll}\text { Experimental Details } & \text { S-7 }\end{array}$

Full Substrate Scope for Sulfoximine Synthesis $\quad$ S-9

$\begin{array}{lr}\text { Analytic Data for Synthesized Compounds } & \text { S-10 }\end{array}$

$\begin{array}{ll}\text { References } & \text { S-25 }\end{array}$

NMR Spectra $\left({ }^{1}\right.$ H NMR and ${ }^{13} \mathbf{C}$ NMR) $\quad$ S-27 


\section{General Considerations}

\section{General Reagent Information}

DMSO was purchased from Sigma Aldrich in sure-seal bottles. $f a c-\operatorname{Ir}(\mathrm{ppy})_{3}$ was synthesized using known procedure. ${ }^{\mathrm{S} 1}$ All other reagents required for the synthesis of 5-aryl-1,2,4-oxadiazolines 1 were purchased from Sigma Aldrich, Alfa Aesar, or TCI chemical companies. Flash column chromatography was performed using Zeochem silica gel 60 (60-200 mesh). Philips CFL $55 \mathrm{~W}$ and EvoluChem blue LEDs 18 W (450-455 nm) were used as light sources.

\section{General Analytical Information}

The synthesized 5-aryl-1,2,4-oxadiazolines (1), sulfoximines (2, 4, 5), and benzimidazoles (3) were characterized by ${ }^{1} \mathrm{H}$ NMR, ${ }^{13} \mathrm{C}$ NMR, and FT-IR spectroscopies, as well as mass spectrometry. NMR spectra were recorded on a Varian $600 \mathrm{MHz}$ instrument $\left(600 \mathrm{MHz}\right.$ for ${ }^{1} \mathrm{H} \mathrm{NMR}$ and $151 \mathrm{MHz}$ for ${ }^{13} \mathrm{C}$ NMR). Copies of ${ }^{1} \mathrm{H}$ NMR and ${ }^{13} \mathrm{C}$ NMR spectra can be found at the end of the Supporting Information. ${ }^{1} \mathrm{H}$ NMR chemical shifts are reported in parts per million (ppm) relative to residual chloroform (7.26 ppm) and dimethylsulfoxide $(2.50 \mathrm{ppm})$ in the deuterated solvent. ${ }^{13} \mathrm{C}$ NMR spectra are reported in ppm relative to deuterochloroform $(77.23 \mathrm{ppm})$ and deuterated dimethylsulfoxide $(39.58 \mathrm{ppm})$ and all were obtained with ${ }^{1} \mathrm{H}$ decoupling. Coupling constants were reported in Hz. FT-IR spectra were recorded on a Nicolet 6700 FT-IR spectrometer (ThermoFisher). Reactions were monitored by thin layer chromatography and GC-MS using the Agilent GC 7890B/5977A inert MSD with Triple-Axis Detector. Mass spectral data of all unknown compounds were acquired at the Korea Basic Science Institute (Daegu) on a Jeol JMS 700 high-resolution mass spectrometer. A quadrupole mass analyzer was used for HRMS measurements. Melting points of unknown compounds were recorded on a Stuart SMP30 apparatus.

\section{Density Functional Theory Calculation Details}

All calculations were performed by using the density functional theory (DFT) with the GAUSSIAN 09 program package ${ }^{\mathrm{S} 2}$. All geometry data were optimized by M062X $\mathrm{X}^{\mathrm{S3}}$ functional using 6-31G(d,p) basis set. Frequency calculation were performed for all stationary points to confirm the local minima, energies and thermal corrections at $298 \mathrm{~K}$. Electronic energies of optimized structure were further calculated by single point calculation of $\mathrm{M} 062 \mathrm{X}$ and 6-311++G(d,p) basis set. Solvent effects were considered at the polarizable continuum model $(\mathrm{PCM})$ method $^{\mathrm{S} 4}$ in dimethylsulfoxide solvent $(\varepsilon=46.8)$. 


\section{Cartesian coordinates, energies, thermal corrections and imaginary frequencies of proposed reaction intermediates and transition states.}

1a

\begin{tabular}{|c|c|c|c|}
\hline \multicolumn{4}{|c|}{ Hartree-Fock Energy = -956.297836 $($ Hartree/Particle $)$} \\
\hline \multicolumn{4}{|c|}{ Zero-point correction $=0.315483$} \\
\hline \multicolumn{4}{|c|}{ Thermal correction to Energy= 0.333146} \\
\hline \multicolumn{4}{|c|}{ Thermal correction to Enthalpy $=0.334090$} \\
\hline \multicolumn{4}{|c|}{ Thermal correction to Gibbs Free Energy $=0.267320$} \\
\hline \multicolumn{4}{|c|}{ Sum of electronic and zero-point Energies $=-955.982353$} \\
\hline \multicolumn{4}{|c|}{ Sum of electronic and thermal Energies $=-955.964690$} \\
\hline \multicolumn{4}{|c|}{ Sum of electronic and thermal Enthalpies $=-955.963746$} \\
\hline \multicolumn{4}{|c|}{ Sum of electronic and thermal Free Energies $=-956.030516$} \\
\hline $\mathrm{C}$ & -2.51837900 & -0.74120400 & 0.35099400 \\
\hline $\mathrm{C}$ & -3.51877500 & 0.18565000 & 0.62738700 \\
\hline $\mathrm{C}$ & -4.70628400 & 0.16976800 & -0.10246000 \\
\hline $\mathrm{C}$ & -4.89212700 & -0.77935100 & -1.10253700 \\
\hline $\mathrm{C}$ & -3.89183400 & -1.71328200 & -1.37425600 \\
\hline $\mathrm{C}$ & -2.70571500 & -1.69338200 & -0.65164800 \\
\hline $\mathrm{H}$ & -3.36419600 & 0.92552200 & 1.40882800 \\
\hline $\mathrm{H}$ & -5.48463800 & 0.89479800 & 0.11253600 \\
\hline $\mathrm{H}$ & -5.81737700 & -0.79572300 & -1.66969800 \\
\hline $\mathrm{H}$ & -4.04053500 & -2.45743300 & -2.15005100 \\
\hline $\mathrm{H}$ & -1.91892600 & -2.41662200 & -0.84400500 \\
\hline $\mathrm{C}$ & -1.20440000 & -0.69128000 & 1.08657700 \\
\hline $\mathrm{H}$ & -1.29509900 & -0.06204200 & 1.99105800 \\
\hline $\mathrm{O}$ & -0.77597000 & -2.00163400 & 1.43403100 \\
\hline $\mathrm{N}$ & -0.08643600 & -0.19662700 & 0.25967700 \\
\hline $\mathrm{N}$ & 0.59384400 & -2.14125700 & 1.15438400 \\
\hline $\mathrm{C}$ & 0.95065900 & -1.09479600 & 0.50445500 \\
\hline $\mathrm{C}$ & 0.10082300 & 1.21496800 & 0.22286200 \\
\hline $\mathrm{C}$ & -0.74383600 & 1.97313600 & -0.58955300 \\
\hline $\mathrm{C}$ & 1.06972700 & 1.84506900 & 1.00685000 \\
\hline $\mathrm{C}$ & -0.61761400 & 3.35865200 & -0.61467500 \\
\hline $\mathrm{H}$ & -1.48476100 & 1.46394100 & -1.19767700 \\
\hline $\mathrm{C}$ & 1.20358200 & 3.22867000 & 0.95827500 \\
\hline $\mathrm{H}$ & 1.71788200 & 1.24734600 & 1.64094600 \\
\hline $\mathrm{C}$ & 0.35821900 & 3.98860800 & 0.15322700 \\
\hline $\mathrm{H}$ & -1.27633900 & 3.94613300 & -1.24595100 \\
\hline $\mathrm{H}$ & 1.96560100 & 3.71425300 & 1.55922200 \\
\hline $\mathrm{H}$ & 0.46209300 & 5.06820000 & 0.12337800 \\
\hline $\mathrm{C}$ & 2.31165100 & -0.94513900 & -0.04006300 \\
\hline $\mathrm{C}$ & 2.54297000 & -0.19593000 & -1.19647200 \\
\hline $\mathrm{C}$ & 3.37341900 & -1.59723700 & 0.59362300 \\
\hline $\mathrm{C}$ & 3.83036800 & -0.10428500 & -1.71432700 \\
\hline $\mathrm{H}$ & 1.71601100 & 0.30491600 & -1.68968200 \\
\hline $\mathrm{C}$ & 4.65689100 & -1.50063400 & 0.07064200 \\
\hline $\mathrm{H}$ & 3.17284800 & -2.17656100 & 1.48873100 \\
\hline $\mathrm{C}$ & 4.88818300 & -0.75297600 & -1.08233200 \\
\hline $\mathrm{H}$ & 4.00735800 & 0.47432600 & -2.61504500 \\
\hline $\mathrm{H}$ & 5.47897400 & -2.00763700 & 0.56512600 \\
\hline $\mathrm{H}$ & 5.89185200 & -0.67530000 & -1.48781500 \\
\hline
\end{tabular}

$1 a^{*}$

Hartree-Fock Energy = -956.207180 (Hartree/Particle)

Zero-point correction $=0.311135$

Thermal correction to Energy $=0.329043$
Thermal correction to Enthalpy $=0.329987$

Thermal correction to Gibbs Free Energy $=0.262626$

Sum of electronic and zero-point Energies $=-955.896045$

Sum of electronic and thermal Energies $=-955.878138$

Sum of electronic and thermal Enthalpies $=-955.877193$

Sum of electronic and thermal Free Energies $=-955.944554$

$\begin{array}{lrrr}\mathrm{C} & 2.28970400 & -0.69558900 & -0.30196700 \\ \mathrm{C} & 3.64068800 & -0.36204100 & -0.35173300 \\ \mathrm{C} & 4.53227200 & -0.91844900 & 0.56336700 \\ \mathrm{C} & 4.07062000 & -1.80976300 & 1.52567500 \\ \mathrm{C} & 2.71695700 & -2.14451400 & 1.57543200 \\ \mathrm{C} & 1.82591000 & -1.58970400 & 0.66606300 \\ \mathrm{H} & 3.99667600 & 0.33462700 & -1.10683800 \\ \mathrm{H} & 5.58396700 & -0.65455700 & 0.52285000 \\ \mathrm{H} & 4.76311500 & -2.24325700 & 2.23988400 \\ \mathrm{H} & 2.35588100 & -2.83889500 & 2.32686800 \\ \mathrm{H} & 0.76923400 & -1.84312500 & 0.70619100 \\ \mathrm{C} & 1.32980600 & -0.10153600 & -1.30449900 \\ \mathrm{H} & 1.81066000 & 0.69112600 & -1.88929100 \\ \mathrm{O} & 0.89019400 & -1.11877300 & -2.22444400 \\ \mathrm{~N} & 0.08561100 & 0.36739200 & -0.73316800 \\ \mathrm{~N} & -0.43191000 & -1.38017700 & -2.11551900 \\ \mathrm{C} & -0.87838600 & -0.57573600 & -0.99422600 \\ \mathrm{C} & -0.08187000 & 1.69778700 & -0.30994200 \\ \mathrm{C} & 1.01246600 & 2.40653000 & 0.19772400 \\ \mathrm{C} & -1.33289400 & 2.32015500 & -0.40964600 \\ \mathrm{C} & 0.85343500 & 3.73132800 & 0.58959000 \\ \mathrm{H} & 1.97478100 & 1.91607500 & 0.30564600 \\ \mathrm{C} & -1.47490300 & 3.63930100 & -0.00244500 \\ \mathrm{H} & -2.17535100 & 1.76616500 & -0.80913000 \\ \mathrm{C} & -0.38732500 & 4.35435000 & 0.49672000 \\ \mathrm{H} & 1.70816100 & 4.27353300 & 0.98110500 \\ \mathrm{H} & -2.44662600 & 4.11522000 & -0.08653600 \\ \mathrm{H} & -0.50783200 & 5.38503600 & 0.81170800 \\ \mathrm{C} & -2.00486900 & -0.92900000 & -0.23505000 \\ \mathrm{C} & -2.28578300 & -0.34599800 & 1.03475000 \\ \mathrm{C} & -2.90418900 & -1.91443900 & -0.73569400 \\ \mathrm{C} & -3.40859900 & -0.72612400 & 1.74256600 \\ \mathrm{H} & -1.59885300 & 0.38073900 & 1.45490400 \\ \mathrm{C} & -4.01813700 & -2.27936600 & -0.00336300 \\ \mathrm{H} & -2.70066000 & -2.36909500 & -1.69881000 \\ \mathrm{C} & -4.28960600 & -1.68868800 & 1.23469300 \\ \mathrm{H} & -3.60064000 & -0.27666000 & 2.71217600 \\ \mathrm{H} & -4.69166800 & -3.03224200 & -0.40113600 \\ \mathrm{H} & -5.17009700 & -1.97604000 & 1.79866900\end{array}$

\section{INT*}

Hartree-Fock Energy = --610.701836 (Hartree/Particle)

Zero-point correction $=0.194497$

Thermal correction to Energy $=0.206253$

Thermal correction to Enthalpy $=0.207198$

Thermal correction to Gibbs Free Energy $=0.154403$

Sum of electronic and zero-point Energies $=-610.507339$

Sum of electronic and thermal Energies $=-610.495583$ 
Sum of electronic and thermal Enthalpies $=-610.494639$

Sum of electronic and thermal Free Energies $=-610.547434$

$\begin{array}{lrrr}\mathrm{N} & 0.76117500 & 1.76441300 & -0.10646400 \\ \mathrm{~N} & -1.19175300 & 2.88641300 & 0.09880100 \\ \mathrm{C} & -0.57929700 & 1.73261800 & -0.00619900 \\ \mathrm{C} & 1.55828500 & 0.64877700 & 0.04282700 \\ \mathrm{C} & 2.82794600 & 0.72656000 & -0.56661300 \\ \mathrm{C} & 1.25050000 & -0.48946600 & 0.82280800 \\ \mathrm{C} & 3.73309400 & -0.31653300 & -0.45722100 \\ \mathrm{H} & 3.06023400 & 1.62459400 & -1.12874500 \\ \mathrm{C} & 2.17753000 & -1.51133900 & 0.95334400 \\ \mathrm{H} & 0.30254900 & -0.54769400 & 1.34487900 \\ \mathrm{C} & 3.41161200 & -1.44038300 & 0.30423200 \\ \mathrm{H} & 4.69834800 & -0.24808000 & -0.94799000 \\ \mathrm{H} & 1.93794200 & -2.37296100 & 1.56793500 \\ \mathrm{H} & 4.12602100 & -2.25055100 & 0.40687700 \\ \mathrm{C} & -1.46967000 & 0.52698100 & -0.08436300 \\ \mathrm{C} & -1.28448200 & -0.42664900 & -1.08943200 \\ \mathrm{C} & -2.53126800 & 0.39504400 & 0.81198400 \\ \mathrm{C} & -2.14960700 & -1.50989800 & -1.18399500 \\ \mathrm{H} & -0.46264500 & -0.31587500 & -1.79051200 \\ \mathrm{C} & -3.39122500 & -0.69564200 & 0.71780400 \\ \mathrm{H} & -2.67507300 & 1.14797400 & 1.58052900 \\ \mathrm{C} & -3.20089100 & -1.64831200 & -0.27861000 \\ \mathrm{H} & -2.00533500 & -2.24759600 & -1.96653600 \\ \mathrm{H} & -4.21082100 & -0.79857200 & 1.42131100 \\ \mathrm{H} & -3.87233800 & -2.49756500 & -0.35350900\end{array}$

\section{INT* ${ }_{\text {rot }}$}

Hartree-Fock Energy = -610.706941 (Hartree/Particle)

Zero-point correction $=0.193797$

Thermal correction to Energy $=0.204761$

Thermal correction to Enthalpy $=0.205705$

Thermal correction to Gibbs Free Energy $=0.154604$

Sum of electronic and zero-point Energies $=-610.513144$

Sum of electronic and thermal Energies $=-610.502181$

Sum of electronic and thermal Enthalpies $=-610.501237$

Sum of electronic and thermal Free Energies $=-610.552337$

$\begin{array}{lrrr}\mathrm{N} & -0.47518900 & 0.42174800 & -0.15248700 \\ \mathrm{~N} & 0.14649500 & -1.82035200 & -0.46929900 \\ \mathrm{C} & 0.44533200 & -0.58794000 & -0.21540600 \\ \mathrm{C} & -1.81821000 & 0.20248000 & -0.03689900 \\ \mathrm{C} & -2.62311200 & 1.35016700 & -0.24724200 \\ \mathrm{C} & -2.46102400 & -1.01529900 & 0.30503100 \\ \mathrm{C} & -4.00168400 & 1.27935800 & -0.16946400 \\ \mathrm{H} & -2.11315500 & 2.27674000 & -0.48776400 \\ \mathrm{C} & -3.84116500 & -1.06942900 & 0.39143300 \\ \mathrm{H} & -1.86770200 & -1.89698100 & 0.51495200 \\ \mathrm{C} & -4.61732700 & 0.06691400 & 0.14841200 \\ \mathrm{H} & -4.60159800 & 2.16500200 & -0.35026900 \\ \mathrm{H} & -4.32305300 & -2.00464900 & 0.65689100 \\ \mathrm{H} & -5.69865800 & 0.00826900 & 0.21876600 \\ \mathrm{C} & 1.88106600 & -0.18797600 & -0.07263000 \\ \mathrm{C} & 2.22637600 & 1.15444200 & 0.08584200 \\ \mathrm{C} & 2.88057800 & -1.16473200 & -0.09481000 \\ \mathrm{C} & 3.56342500 & 1.51538200 & 0.21781000 \\ \mathrm{H} & 1.44043500 & 1.90072100 & 0.10124000 \\ \mathrm{C} & 4.21413300 & -0.79867600 & 0.03747700 \\ \mathrm{H} & 2.60650200 & -2.20811600 & -0.21630000 \\ \mathrm{C} & 4.55876300 & 0.54236400 & 0.19362700\end{array}$

$\begin{array}{rrrr}\mathrm{H} & 3.82777000 & 2.56083300 & 0.34057400 \\ \mathrm{H} & 4.98640200 & -1.56097300 & 0.01806800 \\ \mathrm{H} & 5.60100100 & 0.82705600 & 0.29725700\end{array}$

\section{INT}

Hartree-Fock Energy $=-610.716694($ Hartree/Particle $)$

Zero-point correction $=0.196232$

Thermal correction to Energy $=0.207826$

Thermal correction to Enthalpy $=0.208770$

Thermal correction to Gibbs Free Energy $=0.156353$

Sum of electronic and zero-point Energies $=-610.520466$

Sum of electronic and thermal Energies $=-610.508872$

Sum of electronic and thermal Enthalpies $=-610.507928$

Sum of electronic and thermal Free Energies $=-610.560345$

$\begin{array}{lrrr}\mathrm{N} & 0.70490100 & 1.28103900 & -0.99960000 \\ \mathrm{~N} & 0.02825500 & 2.36181400 & -0.00671800 \\ \mathrm{C} & -0.53532600 & 1.29922500 & -0.32790900 \\ \mathrm{C} & 1.70602700 & 0.49039300 & -0.33034900 \\ \mathrm{C} & 2.65838200 & -0.09062100 & -1.16767900 \\ \mathrm{C} & 1.76287500 & 0.27506900 & 1.04730800 \\ \mathrm{C} & 3.65318100 & -0.90102400 & -0.63309500 \\ \mathrm{H} & 2.59863400 & 0.10967100 & -2.23242700 \\ \mathrm{C} & 2.77014200 & -0.52716600 & 1.57569600 \\ \mathrm{H} & 1.03757600 & 0.74686400 & 1.70159800 \\ \mathrm{C} & 3.71259900 & -1.12192600 & 0.74125900 \\ \mathrm{H} & 4.38900400 & -1.35341600 & -1.29039600 \\ \mathrm{H} & 2.81626100 & -0.68897800 & 2.64806500 \\ \mathrm{H} & 4.49224500 & -1.74958400 & 1.16018400 \\ \mathrm{C} & -1.67878200 & 0.42656600 & -0.16300100 \\ \mathrm{C} & -1.65118300 & -0.82452700 & -0.78131700 \\ \mathrm{C} & -2.77777900 & 0.82826400 & 0.60267100 \\ \mathrm{C} & -2.73311000 & -1.68341800 & -0.62914100 \\ \mathrm{H} & -0.78163800 & -1.10684800 & -1.36744600 \\ \mathrm{C} & -3.85536200 & -0.03487900 & 0.74568900 \\ \mathrm{H} & -2.77451200 & 1.80725200 & 1.07194800 \\ \mathrm{C} & -3.83124600 & -1.28770900 & 0.13179400 \\ \mathrm{H} & -2.72158500 & -2.65938900 & -1.10222900 \\ \mathrm{H} & -4.71524600 & 0.26483400 & 1.33508500 \\ \mathrm{H} & -4.67534800 & -1.95986000 & 0.24829000\end{array}$

\section{PhCHO}

Hartree-Fock Energy $=-345.523222($ Hartree/Particle $)$

Zero-point correction $=0.110427$

Thermal correction to Energy $=0.116689$

Thermal correction to Enthalpy $=0.117633$

Thermal correction to Gibbs Free Energy $=0.079921$

Sum of electronic and zero-point Energies $=-345.412796$

Sum of electronic and thermal Energies $=-345.406535$

Sum of electronic and thermal Enthalpies $=-345.405591$

Sum of electronic and thermal Free Energies $=-345.443302$

$\begin{array}{lrrr}\mathrm{C} & 0.52841600 & 0.21723200 & -0.00004200 \\ \mathrm{C} & -0.36137400 & 1.29064600 & 0.00001300 \\ \mathrm{C} & -1.73285100 & 1.05573000 & -0.00006700 \\ \mathrm{C} & -2.20743100 & -0.25357000 & 0.00002700 \\ \mathrm{C} & -1.31729700 & -1.32922400 & 0.00004200 \\ \mathrm{C} & 0.05082700 & -1.09584200 & -0.00002000 \\ \mathrm{H} & 0.02416200 & 2.30757300 & -0.00017300 \\ \mathrm{H} & -2.42903500 & 1.88777400 & -0.00000300 \\ \mathrm{H} & -3.27698000 & -0.43901600 & 0.00010000 \\ \mathrm{H} & -1.69662500 & -2.34582600 & -0.00005000\end{array}$




$\begin{array}{rrrr}\mathrm{H} & 0.77063200 & -1.90853000 & 0.00006000 \\ \mathrm{C} & 1.98959500 & 0.47127100 & 0.00008300 \\ \mathrm{H} & 2.27808200 & 1.54388100 & 0.00039800 \\ \mathrm{O} & 2.82880600 & -0.39791400 & -0.00006900\end{array}$

\section{DMSO}

Hartree-Fock Energy = -553.163627 (Hartree/Particle)

Zero-point correction $=0.079781$

Thermal correction to Energy $=0.085301$

Thermal correction to Enthalpy $=0.086246$

Thermal correction to Gibbs Free Energy $=0.051625$

Sum of electronic and zero-point Energies $=-553.083847$

Sum of electronic and thermal Energies $=-553.078326$

Sum of electronic and thermal Enthalpies $=-553.077382$

Sum of electronic and thermal Free Energies $=-553.112003$

$\begin{array}{lrrr}\mathrm{S} & -0.00016500 & 0.23535600 & -0.44357800 \\ \mathrm{O} & -0.00190100 & 1.48808400 & 0.38689500 \\ \mathrm{C} & -1.34536100 & -0.80922500 & 0.18211500 \\ \mathrm{H} & -2.28130800 & -0.30149900 & -0.05320700 \\ \mathrm{H} & -1.31857600 & -1.78679000 & -0.30438400 \\ \mathrm{H} & -1.24340100 & -0.90526900 & 1.26547700 \\ \mathrm{C} & 1.34724600 & -0.80629100 & 0.18224800 \\ \mathrm{H} & 1.24553300 & -0.90263700 & 1.26559300 \\ \mathrm{H} & 1.32201800 & -1.78400700 & -0.30415600 \\ \mathrm{H} & 2.28227200 & -0.29707400 & -0.05341300\end{array}$

\section{$\mathrm{TS}_{\mathbf{1}}$}

Imaginary frequency $=-545.91$

Hartree-Fock Energy $=-1163.834140$ (Hartree/Particle)

Zero-point correction $=0.275448$

Thermal correction to Energy $=0.293279$

Thermal correction to Enthalpy $=0.294224$

Thermal correction to Gibbs Free Energy $=0.227018$

Sum of electronic and zero-point Energies $=-1163.558693$

Sum of electronic and thermal Energies $=-1163.540861$

Sum of electronic and thermal Enthalpies $=-1163.539917$

Sum of electronic and thermal Free Energies $=-1163.607123$

$\begin{array}{lrrr}\mathrm{N} & -0.24749400 & -0.38132400 & 0.91905600 \\ \mathrm{C} & 0.57154900 & 0.45136300 & 0.15365600 \\ \mathrm{C} & -0.87650700 & -1.41656900 & 0.34348600 \\ \mathrm{C} & -1.70732600 & -2.22419800 & 1.17260600 \\ \mathrm{C} & -0.75204600 & -1.76024200 & -1.03543200 \\ \mathrm{C} & -2.36387900 & -3.31975300 & 0.65272600 \\ \mathrm{H} & -1.79590500 & -1.94232200 & 2.21631900 \\ \mathrm{C} & -1.41862500 & -2.85765500 & -1.53854200 \\ \mathrm{H} & -0.13624000 & -1.13400600 & -1.67302800 \\ \mathrm{C} & -2.22287200 & -3.64321600 & -0.70258000 \\ \mathrm{H} & -2.99302500 & -3.93087500 & 1.29130000 \\ \mathrm{H} & -1.32396400 & -3.11240400 & -2.58899800 \\ \mathrm{H} & -2.74214500 & -4.50483100 & -1.10941200 \\ \mathrm{C} & 2.01464300 & 0.18593000 & 0.13974500 \\ \mathrm{C} & 2.52711000 & -1.01529000 & 0.65263700 \\ \mathrm{C} & 2.90042800 & 1.12151900 & -0.41938900 \\ \mathrm{C} & 3.89231300 & -1.27161300 & 0.60609800 \\ \mathrm{H} & 1.85036700 & -1.74133500 & 1.09129400 \\ \mathrm{C} & 4.26124400 & 0.85926100 & -0.45840600 \\ \mathrm{H} & 2.49534000 & 2.04599600 & -0.81606900 \\ \mathrm{C} & 4.76499900 & -0.33857800 & 0.05165500\end{array}$

$\begin{array}{rrrr}\mathrm{H} & 4.27688100 & -2.20426300 & 1.00682500 \\ \mathrm{H} & 4.93762600 & 1.59123000 & -0.88888400 \\ \mathrm{H} & 5.83046400 & -0.54150400 & 0.01638100 \\ \mathrm{~N} & 0.09225100 & 1.52274500 & -0.43991900 \\ \mathrm{~S} & -1.48013400 & 2.03534300 & 0.00253800 \\ \mathrm{O} & -2.50209800 & 1.04981200 & -0.43154700 \\ \mathrm{C} & -2.36428000 & 2.85616400 & 1.50132100 \\ \mathrm{H} & -1.73798200 & 3.66650800 & 1.87602000 \\ \mathrm{H} & -3.34488400 & 3.20659800 & 1.17194600 \\ \mathrm{H} & -2.46096900 & 2.05505400 & 2.23284600 \\ \mathrm{C} & -1.49163100 & 3.44959800 & -1.09368600 \\ \mathrm{H} & -1.13329900 & 3.10254600 & -2.06164000 \\ \mathrm{H} & -2.51839000 & 3.80895700 & -1.16458000 \\ \mathrm{H} & -0.82896700 & 4.22039500 & -0.69788600\end{array}$

\section{$\mathbf{T S}_{2}$}

Imaginary frequency $=-640.78$

Hartree-Fock Energy $=-610.667528$ (Hartree/Particle)

Zero-point correction $=0.194003$

Thermal correction to Energy $=0.204877$

Thermal correction to Enthalpy $=0.205822$

Thermal correction to Gibbs Free Energy $=0.155186$

Sum of electronic and zero-point Energies $=-610.473526$

Sum of electronic and thermal Energies $=-610.462651$

Sum of electronic and thermal Enthalpies $=-610.461707$

Sum of electronic and thermal Free Energies $=-610.512342$

$\begin{array}{lrrr}\mathrm{N} & -0.44127600 & 1.07245400 & 0.20594800 \\ \mathrm{~N} & -0.41881300 & -1.21899400 & -0.12221400 \\ \mathrm{C} & 0.25039500 & -0.04405800 & 0.04607200 \\ \mathrm{C} & -1.78529000 & 0.68754200 & 0.25743100 \\ \mathrm{C} & -2.78641900 & 1.38662400 & -0.38301600 \\ \mathrm{C} & -1.99105200 & -0.66280800 & 0.74657600 \\ \mathrm{C} & -4.02430000 & 0.76705500 & -0.60746200 \\ \mathrm{H} & -2.56543200 & 2.37305400 & -0.77631600 \\ \mathrm{C} & -3.24745800 & -1.27158800 & 0.45991700 \\ \mathrm{H} & -1.52135600 & -0.92915100 & 1.69519100 \\ \mathrm{C} & -4.22426000 & -0.56328300 & -0.22008200 \\ \mathrm{H} & -4.81459100 & 1.30747500 & -1.11638500 \\ \mathrm{H} & -3.45267300 & -2.26823100 & 0.83461300 \\ \mathrm{H} & -5.18417500 & -1.03326000 & -0.41059700 \\ \mathrm{C} & 1.72904100 & -0.02671200 & -0.01841300 \\ \mathrm{C} & 2.42047500 & 1.14544900 & 0.30019000 \\ \mathrm{C} & 2.43370600 & -1.17820900 & -0.37995200 \\ \mathrm{C} & 3.80864400 & 1.16277000 & 0.25609600 \\ \mathrm{H} & 1.85396600 & 2.02837500 & 0.57642100 \\ \mathrm{C} & 3.82323600 & -1.15450300 & -0.42264500 \\ \mathrm{H} & 1.88398400 & -2.07974600 & -0.63026000 \\ \mathrm{C} & 4.51143300 & 0.01382400 & -0.10454400 \\ \mathrm{H} & 4.34574300 & 2.07248500 & 0.50394700 \\ \mathrm{H} & 4.37002600 & -2.04791400 & -0.70588900 \\ \mathrm{H} & 5.59621700 & 0.03007300 & -0.13786400\end{array}$

\section{$\mathbf{2 a}$}

Hartree-Fock Energy = -1163.941414 (Hartree/Particle)

Zero-point correction $=0.281745$

Thermal correction to Energy $=0.299105$

Thermal correction to Enthalpy $=0.300049$

Thermal correction to Gibbs Free Energy $=0.235556$

Sum of electronic and zero-point Energies $=-1163.659670$ 
Sum of electronic and thermal Energies $=-1163.642310$

Sum of electronic and thermal Enthalpies $=-1163.641365$

Sum of electronic and thermal Free Energies $=-1163.705859$

$\begin{array}{lrrr}\mathrm{N} & 0.16841400 & -0.96090000 & -0.11140600 \\ \mathrm{C} & 0.51097900 & 0.27938900 & -0.10702700 \\ \mathrm{C} & -1.15454900 & -1.39926600 & 0.05051300 \\ \mathrm{C} & -1.67699400 & -2.32149600 & -0.86373900 \\ \mathrm{C} & -1.93616900 & -1.01719300 & 1.14881900 \\ \mathrm{C} & -2.96604400 & -2.81824500 & -0.70583400 \\ \mathrm{H} & -1.05801900 & -2.63078600 & -1.70048900 \\ \mathrm{C} & -3.21993500 & -1.52563500 & 1.30593100 \\ \mathrm{H} & -1.52208800 & -0.32157200 & 1.87212500 \\ \mathrm{C} & -3.74563200 & -2.42300000 & 0.37855500 \\ \mathrm{H} & -3.36026400 & -3.52487700 & -1.42985100 \\ \mathrm{H} & -3.81308600 & -1.22063900 & 2.16263000 \\ \mathrm{H} & -4.74773500 & -2.81866300 & 0.50580100 \\ \mathrm{C} & -0.40579100 & 1.46366400 & -0.15455700 \\ \mathrm{C} & -1.57797100 & 1.44364600 & -0.91684100 \\ \mathrm{C} & -0.06281500 & 2.62310900 & 0.54506900 \\ \mathrm{C} & -2.39787100 & 2.56510400 & -0.96569000 \\ \mathrm{H} & -1.84539100 & 0.55176700 & -1.47361600 \\ \mathrm{C} & -0.89455000 & 3.73727200 & 0.50933300 \\ \mathrm{H} & 0.86018900 & 2.63552900 & 1.11416500 \\ \mathrm{C} & -2.06315700 & 3.71067800 & -0.24682800 \\ \mathrm{H} & -3.30159100 & 2.54379900 & -1.56594600 \\ \mathrm{H} & -0.62667400 & 4.62871300 & 1.06741300 \\ \mathrm{H} & -2.70960700 & 4.58203500 & -0.28021700 \\ \mathrm{~N} & 1.84453000 & 0.66291900 & -0.09961900 \\ \mathrm{~S} & 2.91099500 & -0.46393300 & 0.15015300 \\ \mathrm{O} & 3.02802500 & -1.15142800 & 1.44139700 \\ \mathrm{C} & 2.91586100 & -1.71303000 & -1.14349400 \\ \mathrm{H} & 2.82093800 & -1.21867000 & -2.11097000 \\ \mathrm{H} & 3.85079300 & -2.26916700 & -1.06120500 \\ \mathrm{H} & 2.05275500 & -2.34463200 & -0.94375600 \\ \mathrm{C} & 4.41340000 & 0.44035900 & -0.16520200 \\ \mathrm{H} & 4.43045100 & 1.25571400 & 0.55812000 \\ \mathrm{H} & 5.25298300 & -0.23645800 & -0.00469400 \\ \mathrm{H} & 4.39304900 & 0.83599500 & -1.18001900\end{array}$

\section{3a'}

Hartree-Fock Energy = -610.740696 (Hartree/Particle)

Zero-point correction $=0.196702$

Thermal correction to Energy $=0.206563$

Thermal correction to Enthalpy $=0.207507$

Thermal correction to Gibbs Free Energy $=0.160975$

Sum of electronic and zero-point Energies $=-610.543995$

Sum of electronic and thermal Energies $=-610.534134$

Sum of electronic and thermal Enthalpies $=-610.533190$

Sum of electronic and thermal Free Energies $=-610.579722$

$\begin{array}{lrrr}\mathrm{N} & -0.50902300 & 1.12350500 & 0.20898900 \\ \mathrm{~N} & -0.43788200 & -1.18579400 & 0.34140500 \\ \mathrm{C} & 0.22253900 & -0.06993700 & 0.21846900 \\ \mathrm{C} & -1.77023000 & 0.71768300 & 0.32493000 \\ \mathrm{C} & -2.92703000 & 1.44292200 & 0.09175300 \\ \mathrm{C} & -1.81618600 & -0.77790400 & 0.53579700 \\ \mathrm{C} & -4.14873600 & 0.69723400 & -0.21642300 \\ \mathrm{H} & -2.89228000 & 2.52237800 & -0.00356800 \\ \mathrm{C} & -2.85081700 & -1.36887600 & -0.36010000 \\ \mathrm{H} & -2.08940800 & -0.98861200 & 1.59029400 \\ \mathrm{C} & -4.06500200 & -0.63016700 & -0.52563100 \\ \mathrm{H} & -5.08539200 & 1.23199000 & -0.31936100\end{array}$

$\begin{array}{rrrr}\mathrm{H} & -2.71541600 & -2.36041000 & -0.77274700 \\ \mathrm{H} & -4.93890800 & -1.14629000 & -0.91310500 \\ \mathrm{C} & 1.68900400 & -0.01895600 & 0.05964500 \\ \mathrm{C} & 2.33741100 & 1.20937100 & -0.08394000 \\ \mathrm{C} & 2.43104500 & -1.20373600 & 0.05597900 \\ \mathrm{C} & 3.72023600 & 1.25095100 & -0.23055000 \\ \mathrm{H} & 1.74601100 & 2.11824000 & -0.08011100 \\ \mathrm{C} & 3.81126600 & -1.15677000 & -0.09169800 \\ \mathrm{H} & 1.90823100 & -2.14737200 & 0.17106600 \\ \mathrm{C} & 4.45815600 & 0.07023800 & -0.23473700 \\ \mathrm{H} & 4.22220900 & 2.20661200 & -0.34256200 \\ \mathrm{H} & 4.38623700 & -2.07724500 & -0.09436200 \\ \mathrm{H} & 5.53712300 & 0.10440500 & -0.34926300\end{array}$

3a

Hartree-Fock Energy = -610.844401 (Hartree/Particle)

Zero-point correction $=0.200028$ (Hartree/Particle)

Thermal correction to Energy $=0.210757$

Thermal correction to Enthalpy $=0.211701$

Thermal correction to Gibbs Free Energy $=0.162288$

Sum of electronic and zero-point Energies $=-610.644374$

Sum of electronic and thermal Energies $=-610.633645$

Sum of electronic and thermal Enthalpies $=-610.632701$

Sum of electronic and thermal Free Energies $=-610.682113$

$\begin{array}{lrrr}\mathrm{N} & -0.47286500 & 1.12389100 & 0.11835200 \\ \mathrm{~N} & -0.52365900 & -1.10266700 & -0.08812200 \\ \mathrm{C} & 0.24635000 & 0.03488800 & 0.01658900 \\ \mathrm{C} & -1.78586000 & 0.69781300 & 0.07307900 \\ \mathrm{C} & -2.97053600 & 1.43911100 & 0.14080700 \\ \mathrm{C} & -1.84338400 & -0.70454800 & -0.06307700 \\ \mathrm{C} & -4.17138100 & 0.75340400 & 0.06427100 \\ \mathrm{H} & -2.93091400 & 2.51740200 & 0.24877000 \\ \mathrm{C} & -3.04807700 & -1.40272000 & -0.14200500 \\ \mathrm{H} & -0.18583400 & -2.03549600 & -0.25775900 \\ \mathrm{C} & -4.20905000 & -0.64704000 & -0.07657600 \\ \mathrm{H} & -5.10596100 & 1.30228100 & 0.11270400 \\ \mathrm{H} & -3.07994000 & -2.48185900 & -0.24902200 \\ \mathrm{H} & -5.17004200 & -1.14750200 & -0.13503800 \\ \mathrm{C} & 1.71531400 & 0.00297500 & 0.01084300 \\ \mathrm{C} & 2.40736800 & 1.20682600 & -0.15635100 \\ \mathrm{C} & 2.43317900 & -1.18475200 & 0.17305700 \\ \mathrm{C} & 3.79561400 & 1.21620700 & -0.17026400 \\ \mathrm{H} & 1.83270400 & 2.11947300 & -0.27226300 \\ \mathrm{C} & 3.82389300 & -1.17158300 & 0.15580300 \\ \mathrm{H} & 1.91573000 & -2.12548700 & 0.34006600 \\ \mathrm{C} & 4.50818600 & 0.02777100 & -0.01816500 \\ \mathrm{H} & 4.32505300 & 2.15414500 & -0.30268100 \\ \mathrm{H} & 4.37201300 & -2.09891200 & 0.28635000 \\ \mathrm{H} & 5.59316700 & 0.03727600 & -0.03079700\end{array}$




\section{Experimental Details}

\section{General experimental procedure for the preparation of 5-aryl-1,2,4-oxadiazoline 1}

An oven-dried 100-mL round-bottom flask equipped with a magnetic stir bar was charged with the oxime $^{\mathrm{S} 5}(2 \mathrm{mmol})$ and $10 \mathrm{~mL}$ of DCM/DMF (9:1 v/v) (or pure DMF for 1i). N-Chlorosuccinimide (2.2 mmol, and $4.4 \mathrm{mmol}$ for $\mathbf{1 j}$ ) was added in portions over $5 \mathrm{~min}$, and the reaction mixture was stirred at room temperature for 1-2 $\mathrm{h}$. The progress of the reaction was monitored by TLC. The solution was then cooled to $0{ }^{\circ} \mathrm{C}$, and the corresponding N-benzylideneaniline ${ }^{\mathrm{S} 6}(2 \mathrm{mmol})$ in $5 \mathrm{~mL}$ DCM was added dropwise followed by the addition of triethylamine $(4 \mathrm{mmol})$. The reaction temperature was maintained at $0-5{ }^{\circ} \mathrm{C}$ for $30 \mathrm{~min}$, and then allowed to reach room temperature with stirring. The progress of the reaction was monitored by TLC. After completion, the volatiles were removed under vacuum. The resulting crude product was diluted with ethyl acetate $(100 \mathrm{~mL})$ and washed with brine $(3 \times 40 \mathrm{~mL})$. The organic layer was dried over anhydrous $\mathrm{MgSO}_{4}$ and concentrated on a rotary evaporator. The desired oxadiazoline 1 was purified by silica-gel flash column chromatography using hexane/EtOAc as the eluent.

\section{General experimental procedure for the synthesis of sulfoxmine 2}

A resealable tube equipped with a magnetic stir bar was charged with the oxadiazoline 1 (0.5 mmol), fac$\operatorname{Ir}(\mathrm{ppy})_{3}(1 \mathrm{~mol} \%, 0.005 \mathrm{mmol})$, and DMSO $(2.5 \mathrm{~mL}, 0.2 \mathrm{M})$. Argon was bubbled through the reaction mixture for $10 \mathrm{~min}$, and the tube was sealed with a silicone septum screw cap. The tube was placed under the light from blue LEDs $(18 \mathrm{~W}, 450-455 \mathrm{~nm})$ at room temperature $\left(20-22{ }^{\circ} \mathrm{C}\right)$. The progress of the reaction was monitored by TLC or gas chromatography. After completion of the reaction, the mixture was diluted with $25 \mathrm{~mL}$ of brine, and was extracted twice with DCM (2 x $25 \mathrm{~mL})$. The combined organic extract was then dried over anhydrous $\mathrm{MgSO}_{4}$, filtered, and concentrated under vacuum. The purification of the reaction mixture by flash column chromatography (hexane/EtOAc) afforded the sulfoxmine 2.

Reaction of 1a to 2a on $3 \mathrm{mmol}$ scale: A 50-mL round-bottom flask with a magnetic stir bar was charged with $1 \mathrm{a}(3.0 \mathrm{mmol}, 0.90 \mathrm{~g}), f a c-\operatorname{Ir}(\mathrm{ppy})_{3}(1 \mathrm{~mol} \%, 20 \mathrm{mg})$, and DMSO (15.0 mL, $\left.0.2 \mathrm{M}\right)$. Argon was bubbled through the reaction mixture for $15 \mathrm{~min}$, and the flask was placed under the light from blue LEDs $(18 \mathrm{~W}, 450-455 \mathrm{~nm})$ at $22{ }^{\circ} \mathrm{C}$. After $24 \mathrm{~h}$, the mixture was diluted with $100 \mathrm{~mL}$ of brine, and was extracted twice with DCM $(2 \times 100 \mathrm{~mL})$. The combined organic extract was then dried over anhydrous $\mathrm{MgSO}_{4}$, filtered, and concentrated under vacuum. The purification of the reaction mixture by flash column chromatography (hexane/EtOAc) afforded 2a in $62 \%$ yield $(0.51 \mathrm{~g})$. 


\section{General experimental procedure for the synthesis of benzimidazole 3}

A resealable tube equipped with a magnetic stir bar was charged with the oxadiazoline 1 ( $0.5 \mathrm{mmol}), \mathrm{fac}$ $\operatorname{Ir}(\mathrm{ppy})_{3}(1 \mathrm{~mol} \%, 0.005 \mathrm{mmol})$, and DMA $(2.5 \mathrm{~mL}, 0.2 \mathrm{M})$. Argon was bubbled through the reaction mixture for $10 \mathrm{~min}$, and the tube was sealed with a silicone septum screw cap. The tube was placed under the light from blue LEDs $(18 \mathrm{~W}, 450-455 \mathrm{~nm})$ at room temperature $\left(20-22{ }^{\circ} \mathrm{C}\right)$. The progress of the reaction was monitored by TLC or gas chromatography. After completion of reaction, the mixture was diluted with $25 \mathrm{~mL}$ brine, and was extracted twice with DCM $(2 \times 25 \mathrm{~mL})$. The combined organic extract was then dried over anhydrous $\mathrm{MgSO}_{4}$, filtered, and concentrated under vacuum. The purification of the reaction mixture by flash column chromatography (hexane/EtOAc) afforded the benzimidazole 3 .

Reaction of 1a to 3a on 3 mmol scale: A 50-mL round-bottom flask with a magnetic stir bar was charged with $1 \mathrm{a}(3.0 \mathrm{mmol}, 0.90 \mathrm{~g}), f a c-\operatorname{Ir}(\mathrm{ppy})_{3}(1 \mathrm{~mol} \%, 20 \mathrm{mg})$, and DMA (15.0 mL, 0.2 M). Argon was bubbled through the reaction mixture for $15 \mathrm{~min}$, and the flask was placed under the light from blue LEDs $(18 \mathrm{~W}, 450-455 \mathrm{~nm})$ at $22{ }^{\circ} \mathrm{C}$. After $15 \mathrm{~h}$, the mixture was diluted with $100 \mathrm{~mL}$ of brine, and was extracted twice with DCM $(2 \times 100 \mathrm{~mL})$. The combined organic extract was then dried over anhydrous $\mathrm{MgSO}_{4}$, filtered, and concentrated under vacuum. The purification of the reaction mixture by flash column chromatography (hexane/EtOAc) afforded 3a in $84 \%$ yield (0.49 g).

\section{General experimental procedure for the synthesis of sulfoxmine derivatives $\mathbf{4}$ and 5}

A resealable tube equipped with a magnetic stir bar was charged with the oxadiazoline 1 (0.3 mmol), fac$\operatorname{Ir}($ ppy) $3(1 \mathrm{~mol} \%, 0.003 \mathrm{mmol})$, and sulfoxides $(1.5 \mathrm{~mL}, 0.2 \mathrm{M})$. Argon was bubbled through the reaction mixture for $10 \mathrm{~min}$, and the tube was sealed with a silicone septum screw cap. The tube was placed under the light from blue LEDs $(18 \mathrm{~W}, 450-455 \mathrm{~nm})$ at room temperature $\left(20-22{ }^{\circ} \mathrm{C}\right)$. The progress of the reaction was monitored by TLC or gas chromatography. After completion of reaction, the mixture was diluted with $25 \mathrm{~mL}$ brine, and was extracted twice with DCM $(2 \times 25 \mathrm{~mL})$. The combined organic extract was then washed with brine $(5 \times 20 \mathrm{~mL})$, dried over anhydrous $\mathrm{MgSO}_{4}$ and concentrated under vacuum. The purification of the reaction mixture by flash column chromatography (hexane/EtOAc) afforded the sulfoximines $\mathbf{4 , 5}$. 


\section{Scheme S1. Full Substrate Scope for Sulfoximine Synthesis ${ }^{a, b}$}
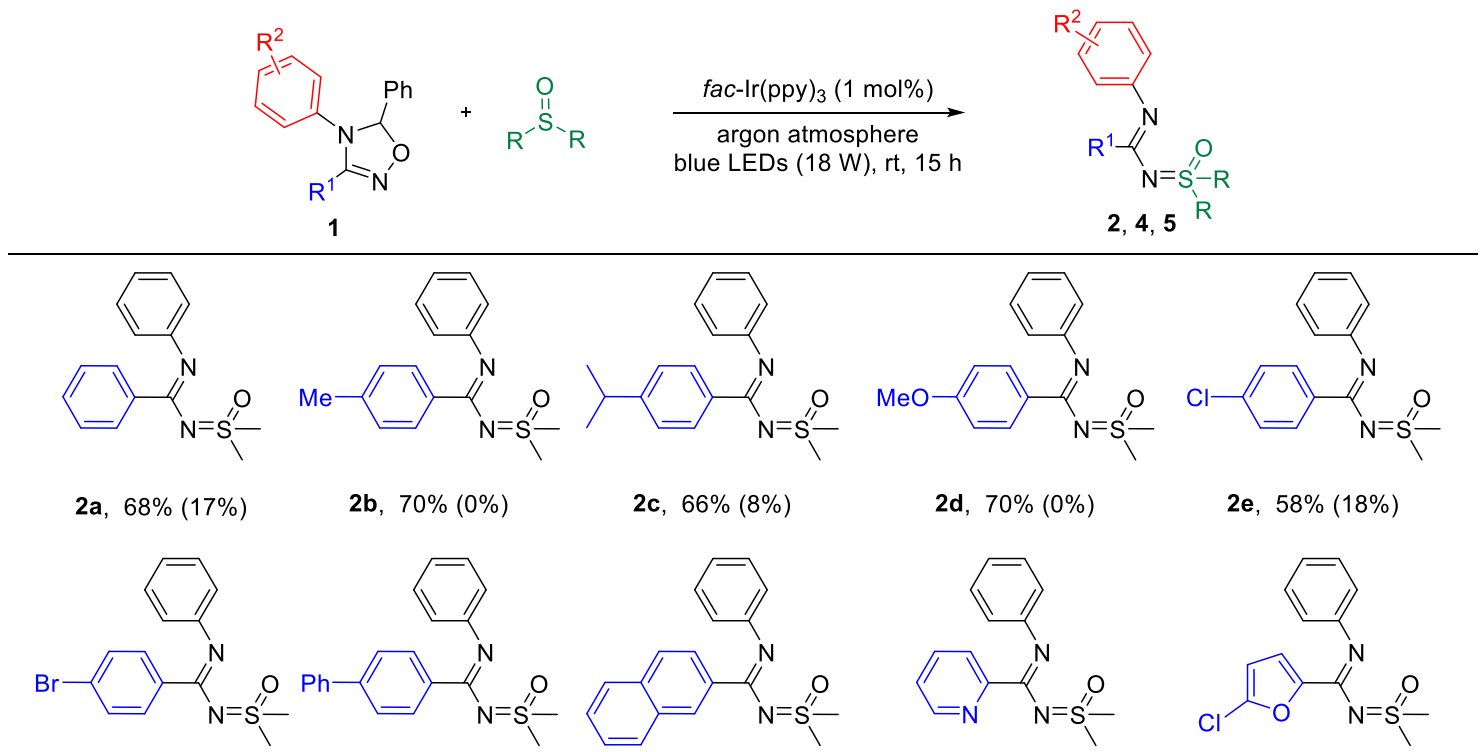
2f, $54 \%(20 \%)$
2g, $60 \%(14 \%)$
2h, $52 \%(20 \%)$
$\mathbf{2 i},{ }^{c} 51 \%(0 \%)$
$\mathbf{2 j},{ }^{c} 49 \%(0 \%)$
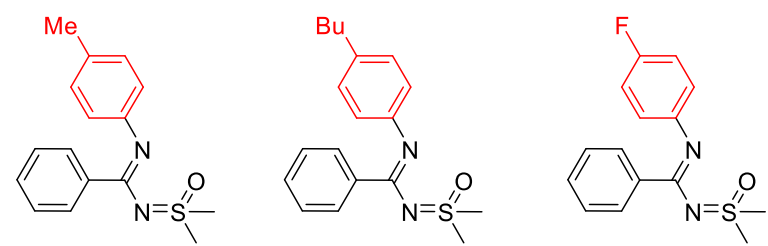

2k, $32 \%(54 \%)$

2I, $32 \%(58 \%)$

2m, 51\% (10\%)

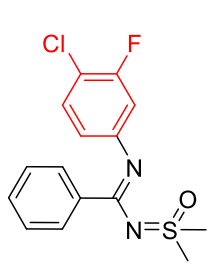

2n, $50 \%(14 \%)$

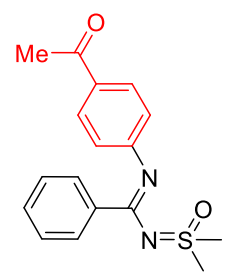

2o, $62 \%(0 \%)$
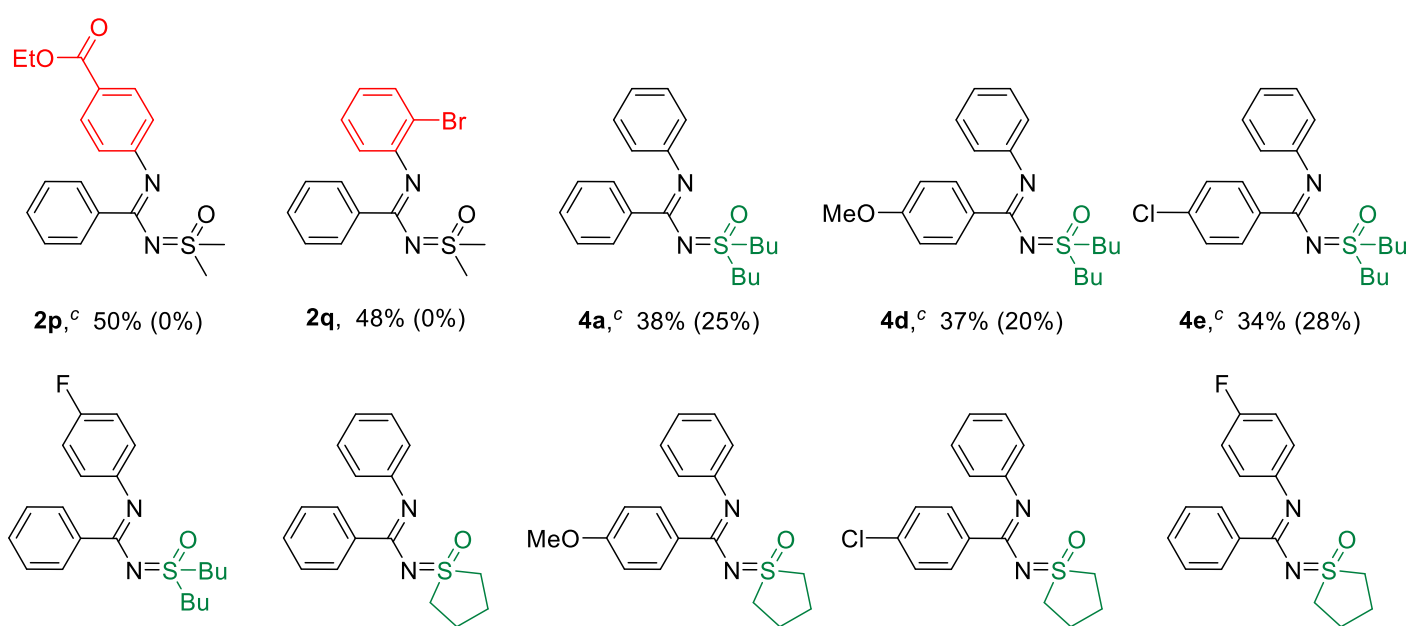

2q, $48 \%(0 \%)$

$\mathbf{4 a},{ }^{c} 38 \%(25 \%)$

4d, ${ }^{c} 37 \%(20 \%)$

$4 e^{c} 34 \%(28 \%)$

$4 m,{ }^{c} \quad 40 \%(21 \%)$

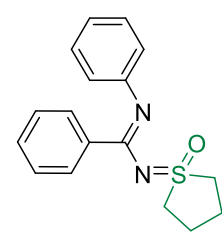

$5 a^{c} 48 \%(18 \%)$

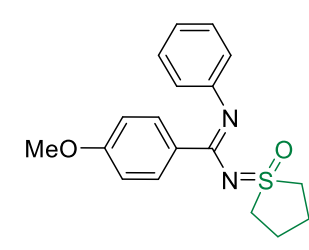

5d, ${ }^{C} 50 \%(7 \%)$

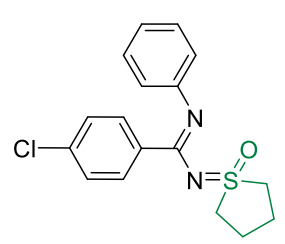

5 e ${ }^{c} 41 \%(15 \%)$

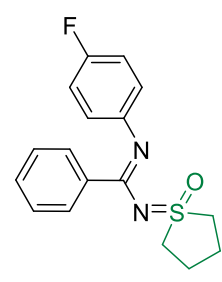

$5 \mathrm{~m},{ }^{c} 43 \%(20 \%)$

${ }^{a}$ Reactions were performed on $0.5 \mathrm{mmol} \mathrm{scale}$, and the isolated yields are reported. ${ }^{b}$ The yield of benzimidazole is given in parentheses. The products are easily separable by flash column chromatography. ${ }^{c} 0.3 \mathrm{mmol}$ scale. 


\section{Analytic Data for Synthesized Compounds}

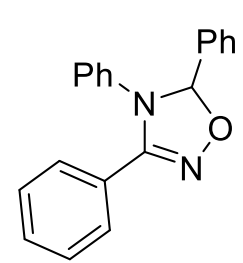

1a (3,4,5-triphenyl-4,5-dihydro-1,2,4-oxadiazole) ${ }^{\mathrm{S7}}$ : white solid; ${ }^{1} \mathrm{H}$ NMR (600 $\mathbf{M H z}$, $\left.\mathbf{C D C l}_{3}\right) \delta 7.64-7.58(\mathrm{~m}, 4 \mathrm{H}), 7.47-7.43(\mathrm{~m}, 3 \mathrm{H}), 7.40(\mathrm{t}, J=7.6 \mathrm{~Hz}, 1 \mathrm{H}), 7.34(\mathrm{dd}, J$ $=7.6,7.6 \mathrm{~Hz}, 2 \mathrm{H}), 7.17(\mathrm{dd}, J=7.8,7.5 \mathrm{~Hz}, 2 \mathrm{H}), 7.09(\mathrm{t}, J=7.5 \mathrm{~Hz}, 1 \mathrm{H}), 6.80(\mathrm{~d}, J=$ $7.8 \mathrm{~Hz}, 2 \mathrm{H}), 6.54$ (s, 1H); ${ }^{13} \mathbf{C}$ NMR (151 MHz, $\left.\mathbf{C D C l}_{3}\right) \delta$ 155.3, 141.5, 139.3, 130.7, 129.9, 129.4, 129.0, 128.9, 128.3, 127.4, 125.7, 125.7, 124.3, 100.5; IR (neat): $v_{\max }=3034,1594,1497$, $1384,650 \mathrm{~cm}^{-1} ; \mathrm{R}_{f} 0.56$ (hexane/EtOAc, 4/1).

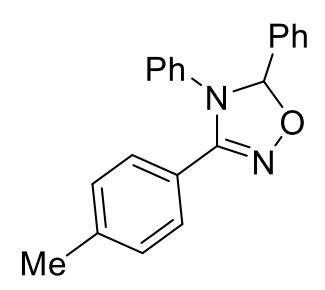

1b (4,5-diphenyl-3-(p-tolyl)-4,5-dihydro-1,2,4-oxadiazole $)^{\mathrm{S7}}:$ pale yellow solid; ${ }^{1} \mathbf{H}$ NMR (600 MHz, $\left.\mathbf{C D C l}_{3}\right) \delta 7.60(\mathrm{dd}, J=7.6,1.7 \mathrm{~Hz}, 2 \mathrm{H}), 7.50(\mathrm{~d}, J=8.1 \mathrm{~Hz}, 2 \mathrm{H})$, $7.46-7.42(\mathrm{~m}, 3 \mathrm{H}), 7.17(\mathrm{dd}, J=8.3,7.4 \mathrm{~Hz}, 2 \mathrm{H}), 7.14(\mathrm{~d}, J=8.1 \mathrm{~Hz}, 2 \mathrm{H}), 7.09$ (tt, $J=7.4,1.4 \mathrm{~Hz}, 1 \mathrm{H}), 6.81(\mathrm{dd}, J=8.3,1.4 \mathrm{~Hz}, 2 \mathrm{H}), 6.52(\mathrm{~s}, 1 \mathrm{H}), 2.35(\mathrm{~s}, 3 \mathrm{H}) ;{ }^{13} \mathrm{C}$ NMR (151 MHz, $\left.\mathbf{C D C l}_{3}\right) \delta 155.3,141.6,141.0,139.4,129.9,129.6,129.3,129.0$, 128.2, 127.4, 125.6, 124.3, 122.7, 100.3, 21.7; IR (neat): $v_{\max }=3035,2920,1611,1594,1495,1391,697$ $\mathrm{cm}^{-1} ; \mathrm{R}_{f} 0.49$ (hexane/EtOAc, 4/1).

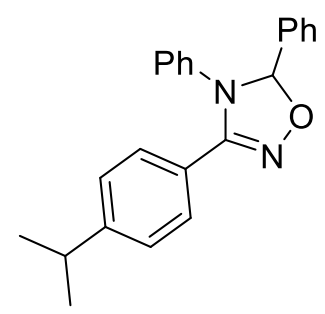

$1 c \quad$ (3-(4-isopropylphenyl)-4,5-diphenyl-4,5-dihydro-1,2,4-oxadiazole) : pale yellow solid, m.p. $75-79{ }^{\circ} \mathrm{C}$; ${ }^{1} \mathbf{H}$ NMR $\left(\mathbf{6 0 0} \mathbf{~ M H z}, \mathbf{C D C l}_{3}\right) \delta 7.61(\mathrm{~d}, J=8.0 \mathrm{~Hz}$, $2 \mathrm{H}), 7.56(\mathrm{~d}, J=8.0 \mathrm{~Hz}, 2 \mathrm{H}), 7.48-7.41(\mathrm{~m}, 3 \mathrm{H}), 7.20(\mathrm{~d}, J=8.1 \mathrm{~Hz}, 2 \mathrm{H}), 7.18$. $(\mathrm{dd}, J=8.6,7.4 \mathrm{~Hz}, 2 \mathrm{H}), 7.10(\mathrm{tt}, J=7.4,1.2 \mathrm{~Hz}, 1 \mathrm{H}), 6.84(\mathrm{dd}, J=8.6,1.2 \mathrm{~Hz}$, 2H), $6.54(\mathrm{~s}, 1 \mathrm{H}), 2.91$ (hept, $J=6.9 \mathrm{~Hz}, 1 \mathrm{H}), 1.25(\mathrm{~d}, J=6.9 \mathrm{~Hz}, 3 \mathrm{H}), 1.24(\mathrm{~d}, J=$ $6.9 \mathrm{~Hz}, 3 \mathrm{H}) ;{ }^{13} \mathbf{C}$ NMR (151 MHz, $\left.\mathbf{C D C l}_{3}\right) \delta$ 155.2, 151.8, 141.7, 139.4, 129.8, 129.3, 129.0, 128.2, 127.3, 126.9, 125.6, 124.2, 123.0, 100.3, 34.2, 23.9; IR (neat): $v_{\max }=3035,2961,1593,1494,1392,695$ $\mathrm{cm}^{-1}$; HRMS m/z (EI) calc. for $\mathrm{C}_{23} \mathrm{H}_{22} \mathrm{~N}_{2} \mathrm{O}\left[\mathrm{M}^{+}\right]$342.1732, found 342.1733; $\mathrm{R}_{f} 0.56$ (hexane/EtOAc, 4/1).<smiles>COc1ccc(C2=NOC(c3ccccc3)N2c2ccccc2)cc1</smiles>

1d (3-(4-methoxyphenyl)-4,5-diphenyl-4,5-dihydro-1,2,4-oxadiazole) $)^{\mathrm{S} 7}$ : orange liquid; ${ }^{1} \mathbf{H}$ NMR (600 MHz, $\left.\mathbf{C D C l}_{3}\right) \delta 7.60(\mathrm{dd}, J=7.9,1.6 \mathrm{~Hz}, 2 \mathrm{H}), 7.55(\mathrm{~d}, J=$ $8.9 \mathrm{~Hz}, 2 \mathrm{H}), 7.47-7.40(\mathrm{~m}, 3 \mathrm{H}), 7.17(\mathrm{dd}, J=8.5,7.4 \mathrm{~Hz}, 2 \mathrm{H}), 7.09(\mathrm{tt}, J=7.4$, $1.2 \mathrm{~Hz}, 1 \mathrm{H}), 6.85(\mathrm{~d}, J=8.9 \mathrm{~Hz}, 2 \mathrm{H}), 6.82(\mathrm{dd}, J=8.5,1.2 \mathrm{~Hz}, 2 \mathrm{H}), 6.52(\mathrm{~s}, 1 \mathrm{H})$, 3.80 (s, 3H); ${ }^{13} \mathbf{C}$ NMR (151 MHz, $\left.\mathbf{C D C l}_{3}\right) \delta$ 161.5, 155.0, 141.7, 139.4, 129.8, 
129.8, 129.3, 129.0, 127.3, 125.6, 124.3, 117.8, 114.3, 100.2, 55.5; IR (neat): $v_{\max }=3035,2936,1610$, 1594, 1494, 1392, 1253, $697 \mathrm{~cm}^{-1} ; \mathrm{R}_{f} 0.31$ (hexane/EtOAc, 4/1).

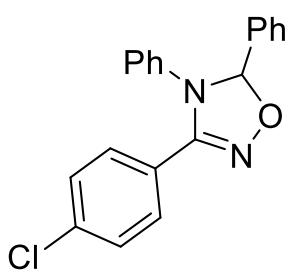

1e (3-(4-chlorophenyl)-4,5-diphenyl-4,5-dihydro-1,2,4-oxadiazole) ${ }^{\mathrm{S} 7}:$ pale yellow solid; ${ }^{1} \mathbf{H}$ NMR (600 MHz, $\left.\mathbf{C D C l}_{3}\right) \delta 7.59(\mathrm{dd}, J=7.4,2.1 \mathrm{~Hz}, 2 \mathrm{H}), 7.55(\mathrm{~d}, J=8.5$ $\mathrm{Hz}, 2 \mathrm{H}), 7.47-7.43(\mathrm{~m}, 3 \mathrm{H}), 7.31(\mathrm{~d}, J=8.5 \mathrm{~Hz}, 2 \mathrm{H}), 7.19(\mathrm{dd}, J=8.2,7.5 \mathrm{~Hz}$, $2 \mathrm{H}), 7.12(\mathrm{tt}, J=7.5,1.3 \mathrm{~Hz}, 1 \mathrm{H}), 6.80(\mathrm{dd}, J=8.2,1.3 \mathrm{~Hz}, 2 \mathrm{H}), 6.53(\mathrm{~s}, 1 \mathrm{H}) ;{ }^{13} \mathrm{C}$ NMR (151 MHz, $\left.\mathbf{C D C l}_{3}\right) \delta 154.5,141.2,139.0,136.8,130.0,129.6,129.5,129.2$, 129.1, 127.3, 126.1, 124.5, 124.2, 100.8; IR (neat): $v_{\max }=3064,1591,1495,1407,735,696 \mathrm{~cm}^{-1} ; \mathrm{R}_{f} 0.62$ (hexane/EtOAc, 4/1).

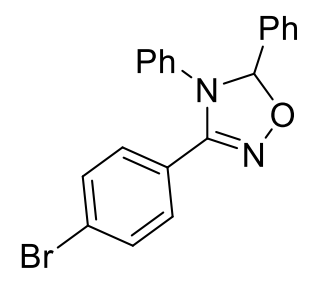

1f (3-(4-bromophenyl)-4,5-diphenyl-4,5-dihydro-1,2,4-oxadiazole) : pale yellow solid, m.p. 138-142 ${ }^{\circ} \mathrm{C} ;{ }^{1} \mathbf{H}$ NMR (600 MHz, $\left.\mathbf{C D C l}_{3}\right) \delta 7.58(\mathrm{dd}, J=7.4,2.1 \mathrm{~Hz}$, 2H), $7.49-7.44(\mathrm{~m}, 7 \mathrm{H}), 7.19(\mathrm{dd}, J=7.6,7.6 \mathrm{~Hz}, 2 \mathrm{H}), 7.12(\mathrm{tt}, J=7.6,1.2 \mathrm{~Hz}$, 1H), $6.80(\mathrm{dd}, J=7.6,1.2 \mathrm{~Hz}, 2 \mathrm{H}), 6.53(\mathrm{~s}, 1 \mathrm{H}) ;{ }^{13} \mathbf{C} \mathbf{~ N M R}\left(\mathbf{1 5 1} \mathbf{~ M H z}, \mathbf{C D C l}_{3}\right) \delta$ 154.6, 141.2, 138.9, 132.2, 130.1, 129.7, 129.6, 129.1, 127.3, 126.1, 125.1, 124.6, 124.5, 100.8; IR (neat): $v_{\max }=3035,1589,1494,1405,695,648 \mathrm{~cm}^{-1}$; HRMS m/z (EI) calc. for $\mathrm{C}_{20} \mathrm{H}_{15} \mathrm{BrN}_{2} \mathrm{O}\left[\mathrm{M}^{+}\right]$378.0368, found 378.0365; $\mathrm{R}_{f} 0.54$ (hexane/EtOAc, 4/1).

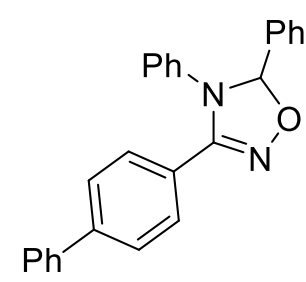

1 (3-([1,1'-biphenyl]-4-yl)-4,5-diphenyl-4,5-dihydro-1,2,4-oxadiazole) : pale yellow solid, m.p. $148-152{ }^{\circ} \mathrm{C} ;{ }^{1} \mathbf{H}$ NMR (600 MHz, $\left.\mathbf{C D C l}_{3}\right) \delta 7.69(\mathrm{~d}, J=8.3 \mathrm{~Hz}$, $2 \mathrm{H}), 7.62(\mathrm{~d}, J=7.6 \mathrm{~Hz}, 2 \mathrm{H}), 7.59-7.56(\mathrm{~m}, 4 \mathrm{H}), 7.46-7.42(\mathrm{~m}, 5 \mathrm{H}), 7.36(\mathrm{t}, J=$ $7.4 \mathrm{~Hz}, 1 \mathrm{H}), 7.20(\mathrm{dd}, J=7.7,7.6 \mathrm{~Hz}, 2 \mathrm{H}), 7.11(\mathrm{t}, J=7.7 \mathrm{~Hz}, 1 \mathrm{H}), 6.85(\mathrm{~d}, J=7.6$ $\mathrm{Hz}, 2 \mathrm{H}), 6.55$ (s, 1H); ${ }^{13} \mathbf{C}$ NMR (151 MHz, $\left.\mathbf{C D C l}_{3}\right) \delta$ 155.1, 143.5, 141.6, 140.2, 139.3, 130.0, 129.5, 129.1, 129.1, 128.7, 128.1, 127.5, 127.4, 127.3, 125.8, 124.5, 124.4, 100.6; IR (neat): $v_{\max }=2987,1611,1598,1494,1382,1090,650 \mathrm{~cm}^{-1}$; HRMS m/z (EI) calc. for $\mathrm{C}_{26} \mathrm{H}_{20} \mathrm{~N}_{2} \mathrm{O}\left[\mathrm{M}^{+}\right] 376.1576$, found $376.1575 ; \mathrm{R}_{f} 0.54$ (hexane/EtOAc, 4/1).

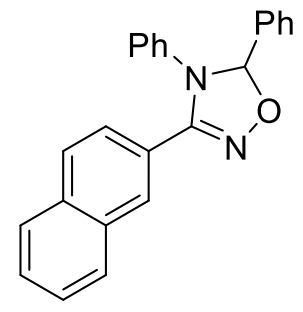

1h (3-(naphthalen-2-yl)-4,5-diphenyl-4,5-dihydro-1,2,4-oxadiazole) ${ }^{\mathrm{S} 7}$ : orange solid; ${ }^{1}$ H NMR (600 MHz, CDCl $) \delta 8.19(\mathrm{~s}, 1 \mathrm{H}), 7.84(\mathrm{~d}, J=6.7 \mathrm{~Hz}, 1 \mathrm{H}), 7.83(\mathrm{~d}, J=$ $8.2 \mathrm{~Hz}, 1 \mathrm{H}), 7.80(\mathrm{~d}, J=8.0 \mathrm{~Hz}, 1 \mathrm{H}), 7.76(\mathrm{~d}, J=8.2 \mathrm{~Hz}, 1 \mathrm{H}), 7.70(\mathrm{~d}, J=7.1 \mathrm{~Hz}$, 2H), $7.53(\mathrm{t}, J=7.2 \mathrm{~Hz}, 1 \mathrm{H}), 7.52-7.48(\mathrm{~m}, 3 \mathrm{H}), 7.47(\mathrm{dd}, J=7.1,6.7 \mathrm{~Hz}, 1 \mathrm{H})$, $7.18(\mathrm{dd}, J=7.9,7.5 \mathrm{~Hz}, 2 \mathrm{H}), 7.10(\mathrm{t}, J=7.5 \mathrm{~Hz}, 1 \mathrm{H}), 6.91(\mathrm{~d}, J=7.9 \mathrm{~Hz}, 2 \mathrm{H}), 6.65$ 
(s, 1H); ${ }^{13} \mathbf{C}$ NMR (151 MHz, $\left.\mathbf{C D C l}_{3}\right) \delta 155.3,141.5,139.2,134.2,132.9,129.9,129.4,129.0,128.7$, 128.6, 128.4, 127.9, 127.5, 127.3, 126.8, 125.7, 124.7, 124.2, 123.0, 100.6; IR (neat): $v_{\max }=3064,2253$, 1597, 1402, 903, $723 \mathrm{~cm}^{-1} ; \mathrm{R}_{f} 0.50$ (hexane/EtOAc, 4/1).

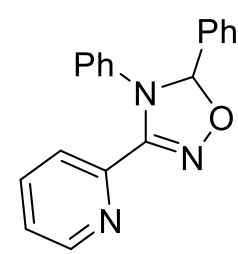

$1 \mathbf{i}$ (4,5-diphenyl-3-(pyridin-2-yl)-4,5-dihydro-1,2,4-oxadiazole) ${ }^{\mathrm{S} 7}$ : light yellow liquid; ${ }^{1} \mathbf{H}$ NMR (600 MHz, $\left.\mathbf{C D C l}_{3}\right) \delta 8.55(\mathrm{ddd}, J=4.8,1.7,1.0 \mathrm{~Hz}, 1 \mathrm{H}), 7.83(\mathrm{ddd}, J=7.9$, $1.1,1.0 \mathrm{~Hz}, 1 \mathrm{H}), 7.74(\mathrm{ddd}, J=7.9,7.6,1.7 \mathrm{~Hz}, 1 \mathrm{H}), 7.63$ (dd, $J=7.5,1.9 \mathrm{~Hz}, 2 \mathrm{H})$, $7.46-7.42(\mathrm{~m}, 3 \mathrm{H}), 7.30$ (ddd, $J=7.6,4.8,1.1 \mathrm{~Hz}, 1 \mathrm{H}), 7.14(\mathrm{dd}, J=7.8,7.4 \mathrm{~Hz}, 2 \mathrm{H})$, $7.07(\mathrm{tt}, J=7.4,1.4 \mathrm{~Hz}, 1 \mathrm{H}), 6.76(\mathrm{dd}, J=7.8,1.4 \mathrm{~Hz}, 2 \mathrm{H}), 6.61(\mathrm{~s}, 1 \mathrm{H}) ;{ }^{13} \mathbf{C} \mathbf{N M R}\left(\mathbf{1 5 1} \mathbf{~ M H z}, \mathbf{C D C l}_{3}\right) \delta$ $154.9,150.6,145.9,141.2,138.7,136.9,130.1,129.2,129.1,127.6,125.5,124.9,124.1,123.9,101.1$; IR (neat): $v_{\max }=3062,1680,1590,1497,1398,696 \mathrm{~cm}^{-1} ; \mathrm{R}_{f} 0.19$ (hexane/EtOAc, 4/1).

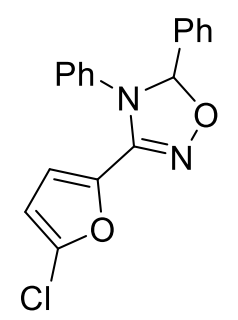

1j (3-(5-chlorofuran-2-yl)-4,5-diphenyl-4,5-dihydro-1,2,4-oxadiazole) ${ }^{\mathrm{S7}}$ : pale yellow solid; ${ }^{1} \mathbf{H}$ NMR (600 MHz, $\left.\mathbf{C D C l}_{3}\right) \delta 7.54(\mathrm{dd}, J=6.5,3.0 \mathrm{~Hz}, 2 \mathrm{H}), 7.44-7.40$ (m, 3H), $7.28(\mathrm{dd}, J=7.6,7.4 \mathrm{~Hz}, 2 \mathrm{H}), 7.22(\mathrm{t}, J=7.4 \mathrm{~Hz}, 1 \mathrm{H}), 6.96(\mathrm{~d}, J=7.6 \mathrm{~Hz}, 2 \mathrm{H}), 6.47(\mathrm{~s}$, 1H), $6.32(\mathrm{~d}, J=3.5 \mathrm{~Hz}, 1 \mathrm{H}), 6.16(\mathrm{~d}, J=3.5 \mathrm{~Hz}, 1 \mathrm{H}) ;{ }^{13} \mathbf{C}$ NMR (151 MHz, $\left.\mathbf{C D C l}_{3}\right) \delta$ $147.6,140.3,139.7,139.3,137.9,130.2,129.7,129.0,127.6,127.2,125.6,115.9,108.4$, 101.0; IR (neat): $v_{\max }=3064,1494,1389,1211,1141,1016,852,758 \mathrm{~cm}^{-1} ; \mathrm{R}_{f} 0.19$ (hexane/EtOAc, 4/1).<smiles>Cc1ccc(N2C(c3ccccc3)=NOC2c2ccccc2)cc1</smiles>

1k (3,5-diphenyl-4-(p-tolyl)-4,5-dihydro-1,2,4-oxadiazole) : pale yellow solid, m.p. $70-74{ }^{\circ} \mathrm{C} ;{ }^{1} \mathbf{H}$ NMR (600 MHz, $\left.\mathbf{C D C l}_{3}\right) \delta 7.61-7.58$ (m, 4H), $7.46-7.43$ (m, 3H), $7.39(\mathrm{t}, J=7.4 \mathrm{~Hz}, 1 \mathrm{H}), 7.32(\mathrm{dd}, J=7.5,7.4 \mathrm{~Hz}, 2 \mathrm{H}), 6.96(\mathrm{~d}, J=8.3 \mathrm{~Hz}, 2 \mathrm{H})$, $6.70(\mathrm{~d}, J=8.3 \mathrm{~Hz}, 2 \mathrm{H}), 6.47(\mathrm{~s}, 1 \mathrm{H}), 2.24(\mathrm{~s}, 3 \mathrm{H}) ;{ }^{13} \mathbf{C} \mathbf{N M R}\left(\mathbf{1 5 1} \mathbf{M H z}, \mathbf{C D C l}_{3}\right)$ $\delta 155.6,139.3,138.8,135.8,130.6,130.0,129.9,129.0,128.8,128.3,127.5,125.7,124.8,100.8,21.1$; IR (neat): $v_{\max }=3036,2987,1597,1513,1467,1382,650 \mathrm{~cm}^{-1} ; \mathrm{HRMS} \mathrm{m} / \mathrm{z}$ (EI) calc. for $\mathrm{C}_{21} \mathrm{H}_{18} \mathrm{~N}_{2} \mathrm{O}\left[\mathrm{M}^{+}\right]$ 314.1419, found 314.1417; $\mathrm{R}_{f} 0.48$ (hexane/EtOAc, 4/1).<smiles>CC(C)(C)c1ccc(N2C(c3ccccc3)=NOC2c2ccccc2)cc1</smiles>

11 (4-(4-butylphenyl)-3,5-diphenyl-4,5-dihydro-1,2,4-oxadiazole) : light yellow liquid; ${ }^{1} \mathbf{H}$ NMR (600 MHz, $\left.\mathbf{C D C l}_{3}\right) \delta 7.70(\mathrm{~d}, J=7.6 \mathrm{~Hz}, 2 \mathrm{H}), 7.65(\mathrm{~d}, J=6.8 \mathrm{~Hz}$, 2H), $7.43(\mathrm{dd}, J=7.4,6.8 \mathrm{~Hz}, 2 \mathrm{H}), 7.41(\mathrm{t}, J=7.2 \mathrm{~Hz}, 1 \mathrm{H}), 7.35(\mathrm{t}, J=7.4 \mathrm{~Hz}$, 1H), $7.29(\mathrm{dd}, J=7.6,7.2 \mathrm{~Hz}, 2 \mathrm{H}), 6.99(\mathrm{~d}, J=8.3 \mathrm{~Hz}, 2 \mathrm{H}), 6.81(\mathrm{~d}, J=8.3 \mathrm{~Hz}$, $2 \mathrm{H}), 6.55(\mathrm{~s}, 1 \mathrm{H}), 2.53(\mathrm{t}, J=7.8 \mathrm{~Hz}, 2 \mathrm{H}), 1.55$ (tt, $J=7.8,7.5 \mathrm{~Hz}, 2 \mathrm{H}), 1.33(\mathrm{tq}, J=7.5,7.4 \mathrm{~Hz}, 2 \mathrm{H})$, $0.93(\mathrm{t}, J=7.4 \mathrm{~Hz}, 3 \mathrm{H}) ;{ }^{13} \mathbf{C}$ NMR (151 MHz, $\left.\mathbf{C D C l}_{3}\right) \delta 155.1,140.2,139.1,138.8,130.2,129.5,129.0$, 
128.6, 128.4, 127.8, 127.0, 125.5, 124.1, 100.3, 34.8, 33.1, 22.1, 13.8; IR (neat): $v_{\max }=3033,2930,1595$, 1499, 1395, $693 \mathrm{~cm}^{-1}$; HRMS m/z (EI) calc. for $\mathrm{C}_{24} \mathrm{H}_{24} \mathrm{~N}_{2} \mathrm{O}\left[\mathrm{M}^{+}\right] 356.1889$, found 356.1886; $\mathrm{R}_{f} 0.54$ (hexane/EtOAc, 4/1).

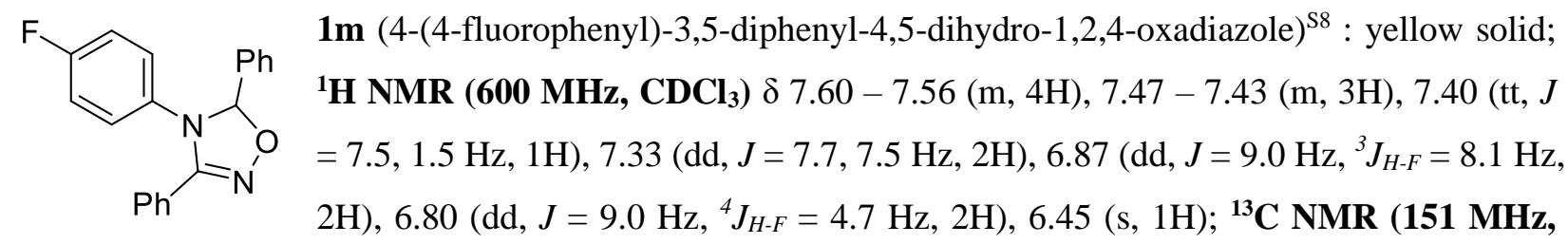
$\left.\mathbf{C D C l}_{3}\right) \delta 160.8\left(\mathrm{~d},{ }^{1} J_{C-F}=246.3 \mathrm{~Hz}\right), 155.6,138.8,137.4,137.4,130.8,130.1,129.0\left(\mathrm{~d},{ }^{2} J_{C-F}=24.8 \mathrm{~Hz}\right)$, 128.2, 127.5, $126.8\left(\mathrm{~d},{ }^{3} J_{C-F}=8.4 \mathrm{~Hz}\right), 125.4,116.4\left(\mathrm{~d},{ }^{2} J_{C-F}=22.8 \mathrm{~Hz}\right), 100.9$; IR (neat): $v_{\max }=3065$, $1592,1506,1394,1220,695 \mathrm{~cm}^{-1} ; \mathrm{R}_{f} 0.56$ (hexane/EtOAc, 4/1).<smiles>Fc1cc(N2C(c3ccccc3)=NOC2c2ccccc2)ccc1Cl</smiles>

1n (4-(4-chloro-3-fluorophenyl)-3,5-diphenyl-4,5-dihydro-1,2,4-oxadiazole) : light yellow liquid; ${ }^{1} \mathbf{H}$ NMR (600 MHz, $\left.\mathbf{C D C l}_{3}\right) \delta 7.65(\mathrm{~d}, J=7.4 \mathrm{~Hz}, 2 \mathrm{H}), 7.61(\mathrm{~d}, J=$ $6.5 \mathrm{~Hz}, 2 \mathrm{H}), 7.48-7.41(\mathrm{~m}, 4 \mathrm{H}), 7.38(\mathrm{dd}, J=7.4,7.3 \mathrm{~Hz}, 2 \mathrm{H}), 7.14(\mathrm{t}, J=8.3 \mathrm{~Hz}$, $1 \mathrm{H}), 6.61\left(\mathrm{~d},{ }^{3} J_{H-F}=9.9 \mathrm{~Hz}, 1 \mathrm{H}\right), 6.58(\mathrm{~s}, 1 \mathrm{H}), 6.54(\mathrm{~d}, J=8.5 \mathrm{~Hz}, 1 \mathrm{H}) ;{ }^{13} \mathbf{C} \mathbf{N M R}$ $\left(151 \mathrm{MHz}, \mathbf{C D C l}_{3}\right) \delta 157.8\left(\mathrm{~d},{ }^{1} J_{C-F}=250.3 \mathrm{~Hz}\right), 154.2,141.1\left(\mathrm{~d},{ }^{3} J_{C-F}=8.7 \mathrm{~Hz}\right)$, 138.2, 131.0, 130.8, 130.1, 129.1, 129.0, 128.0, 127.0, 124.9, $119.9\left(\mathrm{~d},{ }^{3} J_{C-F}=3.2 \mathrm{~Hz}\right), 117.5\left(\mathrm{~d},{ }^{2} J_{C-F}=\right.$ $17.8 \mathrm{~Hz}), 111.9\left(\mathrm{~d},{ }^{2} J_{C-F}=23.5 \mathrm{~Hz}\right), 99.8$; IR (neat): $v_{\max }=3035,1607,1596,1492,1391,1200,724,696$ $\mathrm{cm}^{-1}$; HRMS m/z (EI) calc. for $\mathrm{C}_{20} \mathrm{H}_{14} \mathrm{ClFN}_{2} \mathrm{O}\left[\mathrm{M}^{+}\right]$352.0779, found 352.0779; $\mathrm{R}_{f} 0.48$ (hexane/EtOAc, $4 / 1)$.<smiles>CC(=O)c1ccc(N2C(c3ccccc3)=NOC2c2ccccc2)cc1</smiles>
10 (1-(4-(3,5-diphenyl-1,2,4-oxadiazol-4(5H)-yl)phenyl)ethan-1-one) : pale yellow solid, m.p. $124-127{ }^{\circ} \mathrm{C} ;{ }^{1} \mathbf{H}$ NMR (600 MHz, $\left.\mathbf{C D C l}_{3}\right) \delta 7.75(\mathrm{~d}, J=8.7$ $\mathrm{Hz}, 2 \mathrm{H}), 7.64(\mathrm{~d}, J=7.7 \mathrm{~Hz}, 2 \mathrm{H}), 7.60(\mathrm{~d}, J=7.8 \mathrm{~Hz}, 2 \mathrm{H}), 7.51-7.43(\mathrm{~m}, 4 \mathrm{H})$, $7.40(\mathrm{dd}, J=7.7 \mathrm{~Hz}, 2 \mathrm{H}), 6.78(\mathrm{~d}, J=8.7 \mathrm{~Hz}, 2 \mathrm{H}), 6.68(\mathrm{~s}, 1 \mathrm{H}), 2.50(\mathrm{~s}, 3 \mathrm{H})$; ${ }^{13}$ C NMR (151 MHz, CDCl $\left.\mathbf{3}\right) \delta$ 195.8, 153.1, 144.3, 137.6, 130.2, 129.3, 128.7, 128.3, 128.2, 127.2, 126.0, 124.4, 120.8, 109.2, 98.4, 25.6; IR (neat): $v_{\max }=3065,2988,1680,1601$, 1561, 1469, 1384, 1269, $650 \mathrm{~cm}^{-1}$; HRMS m/z (EI) calc. for $\mathrm{C}_{22} \mathrm{H}_{18} \mathrm{~N}_{2} \mathrm{O}_{2}\left[\mathrm{M}^{+}\right]$342.1368, found 342.1367; $\mathrm{R}_{f} 0.22$ (hexane/EtOAc, 4/1). 
<smiles>CCOC(=O)c1ccc(N2C(c3ccccc3)=NOC2c2ccccc2)cc1</smiles>

$1 p$ (ethyl 4-(3,5-diphenyl-1,2,4-oxadiazol-4(5H)-yl)benzoate) : light yellow liquid; ${ }^{1} \mathbf{H}$ NMR (600 MHz, $\left.\mathbf{C D C l}_{3}\right) \delta 7.80(\mathrm{~d}, J=8.7 \mathrm{~Hz}, 2 \mathrm{H}), 7.63(\mathrm{~d}, J=7.1$ $\mathrm{Hz}, 2 \mathrm{H}), 7.58(\mathrm{~d}, J=6.7 \mathrm{~Hz}, 2 \mathrm{H}), 7.39(\mathrm{dd}, J=7.2,6.7 \mathrm{~Hz}, 2 \mathrm{H}), 7.38(\mathrm{t}, J=7.9$ $\mathrm{Hz}, 1 \mathrm{H}), 7.36(\mathrm{t}, J=7.2 \mathrm{~Hz}, 1 \mathrm{H}), 7.32(\mathrm{dd}, J=7.9,7.2 \mathrm{~Hz}, 2 \mathrm{H}), 6.76(\mathrm{~d}, J=8.7$ $\mathrm{Hz}, 2 \mathrm{H}), 6.67(\mathrm{~s}, 1 \mathrm{H}), 4.25(\mathrm{q}, J=7.1 \mathrm{~Hz}, 2 \mathrm{H}), 1.27(\mathrm{t}, J=7.1 \mathrm{~Hz}, 3 \mathrm{H}) ;{ }^{13} \mathbf{C}$ NMR (151 MHz, $\left.\mathbf{C D C l}_{3}\right) \delta 165.6,153.9,144.8,138.5,130.9,130.6,129.9,129.0,128.9,128.0,126.9$, 126.3, 125.2, 121.7, 99.2, 60.9, 14.3; IR (neat): $v_{\max }=3066,2983,1709,1607,1594,1498,1390,1275$, $694 \mathrm{~cm}^{-1}$; HRMS m/z (EI) calc. for $\mathrm{C}_{23} \mathrm{H}_{20} \mathrm{~N}_{2} \mathrm{O}_{3}\left[\mathrm{M}^{+}\right]$372.1474, found 372.1477; $\mathrm{R}_{f} 0.37$ (hexane/EtOAc, 4/1).

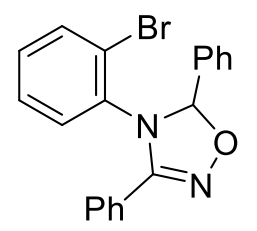

1q (4-(2-bromophenyl)-3,5-diphenyl-4,5-dihydro-1,2,4-oxadiazole) : orange liquid; ${ }^{1} \mathbf{H}$ NMR (600 MHz, CDCl $) \delta 7.63$ - 7.44 (m, 5H), 7.37 - 7.31 (m, 4H), 7.27 (dd, $J=7.4$, $7.4 \mathrm{~Hz}, 2 \mathrm{H}), 7.19-6.41(\mathrm{~m}, 4 \mathrm{H}) ;{ }^{13} \mathbf{C}$ NMR (151 MHz, $\left.\mathbf{C D C l}_{3}\right) \delta$ 155.4, 141.5, 137.0, $133.7,132.1,130.4,129.6,129.0,128.4,128.3,127.8,127.7,125.1,123.0,101.1,98.0$; IR (neat): $v_{\max }=3065,1597,1476,1398,694,647 \mathrm{~cm}^{-1}$; HRMS m/z (EI) calc. for $\mathrm{C}_{20} \mathrm{H}_{15} \mathrm{BrN}_{2} \mathrm{O}\left[\mathrm{M}^{+}\right]$ 378.0368, found 378.0369; $\mathrm{R}_{f} 0.38$ (hexane/EtOAc, 4/1).<smiles>COc1ccc(N2C(c3ccccc3)=NOC2c2ccccc2)cc1</smiles>

$1 r$ (4-(4-methoxyphenyl)-3,5-diphenyl-4,5-dihydro-1,2,4-oxadiazole) ${ }^{\mathrm{S} 9}$ : light yellow liquid; ${ }^{1} \mathbf{H}$ NMR (600 MHz, $\left.\mathbf{C D C l}_{3}\right) \delta 7.62$ - $7.56(\mathrm{~m}, 4 \mathrm{H}), 7.46-7.41$ (m, 3H), 7.37 (t, $J=7.4 \mathrm{~Hz}, 1 \mathrm{H}), 7.30$ (dd, $J=7.8,7.4 \mathrm{~Hz}, 2 \mathrm{H}), 6.79$ (d, $J=8.8$ $\mathrm{Hz}, 2 \mathrm{H}), 6.70(\mathrm{~d}, J=8.8 \mathrm{~Hz}, 2 \mathrm{H}), 6.42(\mathrm{~s}, 1 \mathrm{H}), 3.70(\mathrm{~s}, 3 \mathrm{H}) ;{ }^{13} \mathbf{C}$ NMR (151 MHz, $\left.\mathbf{C D C l}_{3}\right) \delta 158.0,156.0,139.1,134.0,130.5,129.9,128.9,128.7,128.2,127.6,127.1,125.6,114.7$, 101.2, 55.5; IR (neat): $v_{\max }=3067,2933,1608,1596,1510,1399,694 \mathrm{~cm}^{-1} ; \mathrm{R}_{f} 0.56$ (hexane/EtOAc, 4/1).<smiles>Cc1ccc(N2C(c3ccccc3)=NOC2c2ccccc2)c(C)c1</smiles>
1 s (4-(2,4-dimethylphenyl)-3,5-diphenyl-4,5-dihydro-1,2,4-oxadiazole) : orange liquid; ${ }^{1} \mathbf{H}$ NMR (600 MHz, $\left.\mathbf{C D C l}_{3}\right) \delta 7.54-7.51(\mathrm{~m}, 5 \mathrm{H}), 7.38-7.37(\mathrm{~m}, 4 \mathrm{H})$, $7.31(\mathrm{dd}, J=7.4,7.4 \mathrm{~Hz}, 2 \mathrm{H}), 7.24(\mathrm{~d}, J=7.7 \mathrm{~Hz}, 2 \mathrm{H}), 6.91(\mathrm{~s}, 1 \mathrm{H}), 2.21(\mathrm{~s}, 6 \mathrm{H})$; ${ }^{13}$ C NMR (151 MHz, $\left.\mathbf{C D C l}_{3}\right) \delta 132.2,132.1,130.3,129.7,128.6,128.6,128.5$, 128.5, 128.4, 128.4, 128.3, 128.2, 127.7, 127.6, 127.6, 125.6, 21.0, 18.0; IR (neat): $v_{\max }=3035,2923$, 1595, 1501, 1446, 1371, $693 \mathrm{~cm}^{-1}$; HRMS m/z (EI) calc. for $\mathrm{C}_{22} \mathrm{H}_{20} \mathrm{~N}_{2} \mathrm{O}\left[\mathrm{M}^{+}\right]$328.1576, found 328.1572; $\mathrm{R}_{f} 0.46$ (hexane/EtOAc, 4/1). 


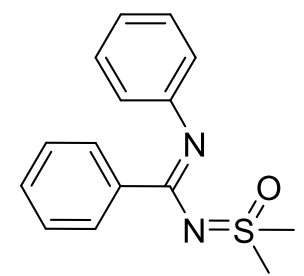

2a ( $N$-(dimethyl(oxo)- $\lambda^{6}$-sulfanylidene)- $N^{\prime}$-phenylbenzimidamide) ${ }^{\mathrm{S} 10}:$ pale yellow solid (93 mg, 68\%); ${ }^{1} \mathbf{H}$ NMR (600 MHz, $\left.\mathbf{C D C l}_{3}\right) \delta 7.33$ (d, $\left.J=7.5 \mathrm{~Hz}, 2 \mathrm{H}\right), 7.22(\mathrm{t}$, $J=7.3 \mathrm{~Hz}, 1 \mathrm{H}), 7.18(\mathrm{dd}, J=7.5,7.3 \mathrm{~Hz}, 2 \mathrm{H}), 7.12(\mathrm{dd}, J=7.7,7.5 \mathrm{~Hz}, 2 \mathrm{H}), 6.89$ (t, $J=7.5 \mathrm{~Hz}, 1 \mathrm{H}), 6.68(\mathrm{~d}, J=7.7 \mathrm{~Hz}, 2 \mathrm{H}), 3.44(\mathrm{~s}, 6 \mathrm{H}) ;{ }^{13} \mathbf{C}$ NMR (151 MHz, $\left.\mathbf{C D C l}_{3}\right)$ $\delta 160.6,150.3,135.6,129.6,129.2,128.8,127.9,122.3,122.3,41.8 ;$ IR (neat): $v_{\max }$ $=3019,2928,1597,1572,1329,1210,1126,698 \mathrm{~cm}^{-1} ; \mathrm{R}_{f} 0.39$ (hexane/EtOAc, 1/2).

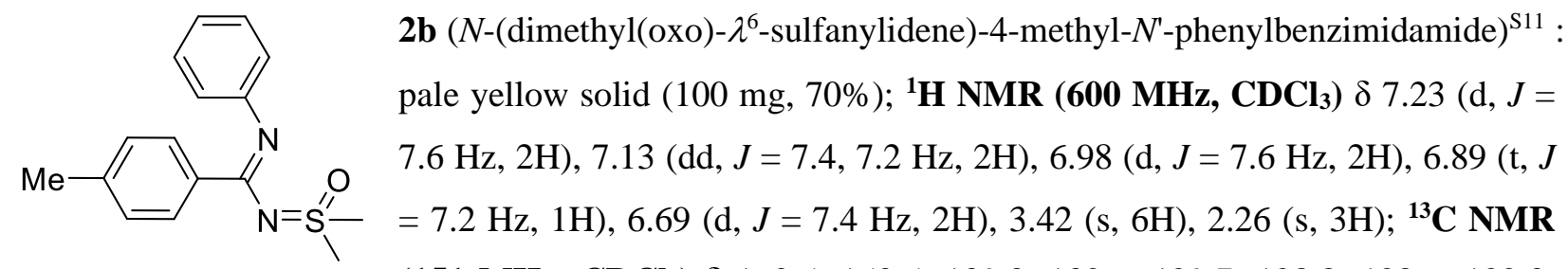
(151 MHz, $\left.\mathbf{C D C l}_{3}\right) \delta 160.5,150.5,139.3,132.6,129.7,128.8,128.6,122.3$, 122.1, 41.8, 21.5; IR (neat): $v_{\max }=3029,2929,1587,1569,1483,1293,1212,1127,649 \mathrm{~cm}^{-1} ; \mathrm{R}_{f} 0.40$ (hexane/EtOAc, 1/2).

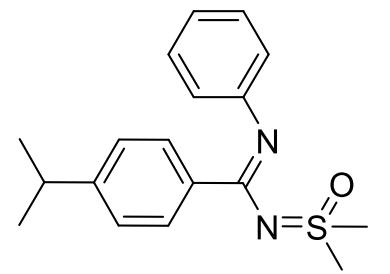

2c ( $N$-(dimethyl(oxo)- $\lambda^{6}$-sulfanylidene)-4-isopropyl- $N$ '-phenylbenzimidamide) : colorless solid (103 mg, 66\%), m.p. 136-140 ${ }^{\circ} \mathrm{C} ;{ }^{1} \mathbf{H}$ NMR (600 $\left.\mathbf{M H z}, \mathbf{C D C l}_{3}\right) \delta$ $7.27(\mathrm{~d}, J=7.6 \mathrm{~Hz}, 2 \mathrm{H}), 7.13(\mathrm{dd}, J=7.3,7.0 \mathrm{~Hz}, 2 \mathrm{H}), 7.03(\mathrm{~d}, J=7.6 \mathrm{~Hz}, 2 \mathrm{H})$, 6.90 (t, $J=7.0 \mathrm{~Hz}, 1 \mathrm{H}), 6.70(\mathrm{~d}, J=7.3 \mathrm{~Hz}, 2 \mathrm{H}), 3.43$ (s, 6H), 2.81 (sept, $J=$ $6.7 \mathrm{~Hz}, 1 \mathrm{H}), 1.17(\mathrm{~d}, J=6.7 \mathrm{~Hz}, 6 \mathrm{H}) ;{ }^{13} \mathbf{C} \mathbf{N M R}\left(151 \mathbf{M H z}, \mathbf{C D C l}_{3}\right) \delta 160.4$, $150.5,150.2,132.9,129.8,128.8,126.0,122.3,122.1,41.8,34.1,24.0$; IR (neat): $v_{\max }=2964,2253$, 1587, 1214, 1024, 903, $723 \mathrm{~cm}^{-1}$; HRMS m/z (EI) calc. for $\mathrm{C}_{18} \mathrm{H}_{22} \mathrm{~N}_{2} \mathrm{OS}\left[\mathrm{M}^{+}\right]$314.1453, found 314.1456; $\mathrm{R}_{f} 0.42$ (hexane/EtOAc, 1/2).

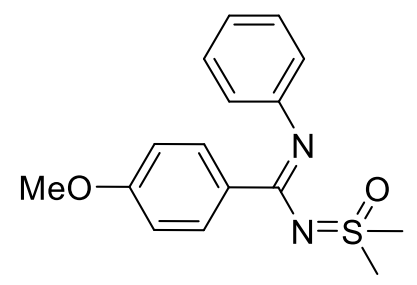

2d ( $N$-(dimethyl(oxo)- $\lambda^{6}$-sulfanylidene)-4-methoxy- $N$ '-phenylbenzimidamide) ${ }^{\mathrm{S} 11}$ : white solid (105 mg, 70\%); ${ }^{1} \mathbf{H}$ NMR (600 MHz, $\left.\mathbf{C D C l}_{3}\right) \delta 7.31(\mathrm{~d}, J$ $=8.0 \mathrm{~Hz}, 2 \mathrm{H}), 7.14(\mathrm{dd}, J=6.9,6.0 \mathrm{~Hz}, 2 \mathrm{H}), 6.90(\mathrm{t}, J=6.0 \mathrm{~Hz}, 1 \mathrm{H}), 6.70(\mathrm{~d}$, $J=6.9 \mathrm{~Hz}, 2 \mathrm{H}), 6.68(\mathrm{~d}, J=8.0 \mathrm{~Hz}, 2 \mathrm{H}), 3.74(\mathrm{~s}, 3 \mathrm{H}), 3.41(\mathrm{~s}, 6 \mathrm{H}) ;{ }^{13} \mathrm{C}$ NMR (151 MHz, CDCl $) \delta 160.3,159.8,150.6,131.5,128.8,127.7,122.2$, 122.1, 113.1, 55.3, 41.8; IR (neat): $v_{\max }=3153,2935,1606,1588,1509,1250,1214,1024,649 \mathrm{~cm}^{-1} ; \mathrm{R}_{f}$ 0.27 (hexane/EtOAc, 1/2). 


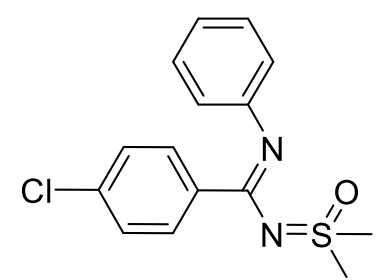

2e (4-chloro- $N$-(dimethyl(oxo)- $\lambda^{6}$-sulfanylidene)- $N$-phenylbenzimidamide) ${ }^{\mathrm{S} 11}$ : pale yellow solid (89 mg, 58\%); ${ }^{1} \mathbf{H}$ NMR (600 $\left.\mathbf{~ M H z , ~} \mathbf{C D C l}_{3}\right) \delta 7.28(\mathrm{~d}, J=8.9$ $\mathrm{Hz}, 2 \mathrm{H}), 7.15(\mathrm{~d}, J=8.9 \mathrm{~Hz}, 2 \mathrm{H}), 7.13(\mathrm{dd}, J=8.0,7.8 \mathrm{~Hz}, 2 \mathrm{H}), 6.91(\mathrm{t}, J=7.8$ $\mathrm{Hz}, 1 \mathrm{H}), 6.66(\mathrm{~d}, J=8.0 \mathrm{~Hz}, 2 \mathrm{H}), 3.43(\mathrm{~s}, 6 \mathrm{H}) ;{ }^{13} \mathbf{C ~ N M R}\left(151 \mathbf{M H z}, \mathbf{C D C l}_{3}\right) \delta$ 159.3, 150.0, 135.2, 134.0, 131.2, 129.0, 128.2, 122.5, 122.2, 41.8; IR (neat): $v_{\max }=3154,1601,1587,1488,1290,1217,1128,650 \mathrm{~cm}^{-1} ; \mathrm{R}_{f} 0.42$ (hexane/EtOAc, 1/2).

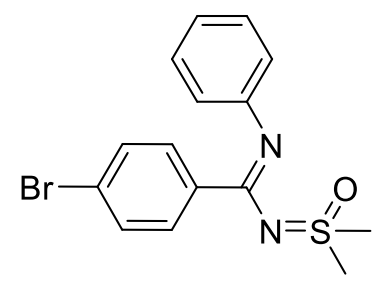

$2 f$ (4-bromo- $N$-(dimethyl(oxo)- $\lambda^{6}$-sulfanylidene)- $N$-phenylbenzimidamide) : pale yellow solid (94 mg, 54\%), m.p. 135-139 ${ }^{\circ} \mathrm{C} ;{ }^{1} \mathbf{H}$ NMR (600 MHz, $\left.\mathbf{C D C l}_{3}\right)$ $\delta 7.31(\mathrm{~d}, J=8.0 \mathrm{~Hz}, 2 \mathrm{H}), 7.20(\mathrm{~d}, J=8.0 \mathrm{~Hz}, 2 \mathrm{H}), 7.14(\mathrm{dd}, J=7.4,7.5 \mathrm{~Hz}$, 2H), $6.91(\mathrm{t}, J=7.4 \mathrm{~Hz}, 1 \mathrm{H}), 6.66(\mathrm{~d}, J=7.5 \mathrm{~Hz}, 2 \mathrm{H}), 3.43(\mathrm{~s}, 6 \mathrm{H}) ;{ }^{13} \mathbf{C}$ NMR (151 MHz, $\left.\mathbf{C D C l}_{3}\right) \delta 159.4,149.9,134.4,131.4,131.1,129.0,123.6,122.6$, 122.2, 41.8; IR (neat): $v_{\max }=3155,2254,1585,1217,1024,903,723 \mathrm{~cm}^{-1}$; HRMS m/z (EI) calc. for $\mathrm{C}_{15} \mathrm{H}_{15} \mathrm{BrN}_{2} \mathrm{OS}\left[\mathrm{M}^{+}\right]$350.0088, found 350.0091; $\mathrm{R}_{f} 0.37$ (hexane/EtOAc, 1/2).

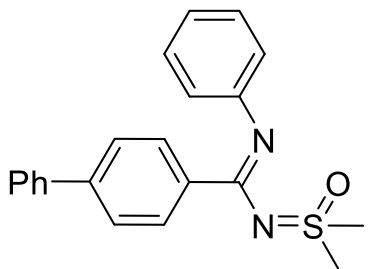

$2 \mathrm{~g}$ ( $N$-(dimethyl(oxo)- $\lambda^{6}$-sulfanylidene)- $N^{\prime}$-phenyl-[1,1'-biphenyl]-4-carboximidamide) : pale yellow solid (104 mg, 60\%), m.p. 169-174 ${ }^{\circ} \mathrm{C} ;{ }^{1} \mathbf{H}$ NMR (600 MHz, $\left.\mathbf{C D C l}_{3}\right) \delta 7.53(\mathrm{~d}, J=7.1 \mathrm{~Hz}, 2 \mathrm{H}), 7.42-7.39(\mathrm{~m}, 6 \mathrm{H}), 7.32(\mathrm{t}, J=7.1$ $\mathrm{Hz}, 1 \mathrm{H}), 7.15(\mathrm{dd}, J=7.2,6.9 \mathrm{~Hz}, 2 \mathrm{H}), 6.92(\mathrm{t}, J=6.9 \mathrm{~Hz}, 1 \mathrm{H}), 6.73(\mathrm{~d}, J=7.2$ $\mathrm{Hz}, 2 \mathrm{H}), 3.46(\mathrm{~s}, 6 \mathrm{H}) ;{ }^{13} \mathbf{C}$ NMR (151 MHz, $\left.\mathbf{C D C l}_{3}\right) \delta 160.2,150.3,141.9$, 140.6, 134.4, 130.2, 128.9, 128.9, 127.7, 127.3, 126.6, 110.2, 110.1, 41.8; IR (neat): $v_{\max }=3029,2253$, 1586, 1293, 1128, 1025, 905, $728 \mathrm{~cm}^{-1}$; HRMS m/z (EI) calc. for $\mathrm{C}_{21} \mathrm{H}_{20} \mathrm{~N}_{2} \mathrm{OS}$ [M+] 348.1296, found 348.1299; $\mathrm{R}_{f} 0.40$ (hexane/EtOAc, 1/2).

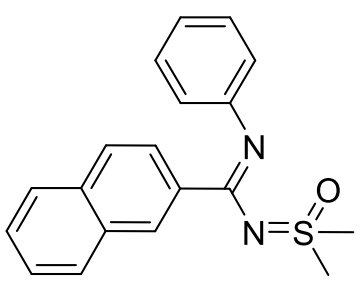

2h ( $N$-(dimethyl(oxo)- $\lambda^{6}$-sulfanylidene)- $N^{\prime}$-phenyl-2-naphthimidamide) : colorless solid (84 mg, 52\%), m.p. 135-140 ${ }^{\circ} \mathrm{C} ;{ }^{1} \mathbf{H}$ NMR (600 MHz, $\left.\mathbf{C D C l}_{3}\right) \delta$ $7.99(\mathrm{~s}, 1 \mathrm{H}), 7.73(\mathrm{~d}, J=7.2 \mathrm{~Hz}, 2 \mathrm{H}), 7.58(\mathrm{~d}, J=8.4 \mathrm{~Hz}, 1 \mathrm{H}), 7.45$ (dd, $J=8.5$, $7.2 \mathrm{~Hz}, 1 \mathrm{H}), 7.42(\mathrm{dd}, J=8.5,7.2 \mathrm{~Hz}, 1 \mathrm{H}), 7.30(\mathrm{~d}, J=8.4 \mathrm{~Hz}, 1 \mathrm{H}), 7.10(\mathrm{dd}, J$ $=7.3,7.1 \mathrm{~Hz}, 2 \mathrm{H}), 6.88(\mathrm{t}, J=7.1 \mathrm{~Hz}, 1 \mathrm{H}), 6.72(\mathrm{~d}, J=7.3 \mathrm{~Hz}, 2 \mathrm{H}), 3.48(\mathrm{~s}$, 6H); ${ }^{13} \mathbf{C}$ NMR (151 MHz, CDCl $) \delta 160.5,150.2,133.6,133.1,132.9,130.1,129.0,128.9,128.8,127.7$, 127.2, 127.0, 126.7, 126.2, 122.4, 41.8; IR (neat): $v_{\max }=2254,1585,1216,1024,903,723 \mathrm{~cm}^{-1}$; HRMS $\mathrm{m} / \mathrm{z}$ (EI) calc. for $\mathrm{C}_{19} \mathrm{H}_{18} \mathrm{~N}_{2} \mathrm{OS}\left[\mathrm{M}^{+}\right]$322.1140, found 322.1143; $\mathrm{R}_{f} 0.42$ (hexane/EtOAc, 1/2). 


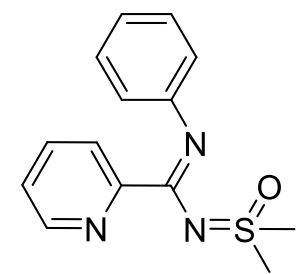

2i ( $N$-(dimethyl(oxo)- $\lambda^{6}$-sulfanylidene)- $N$-phenylpicolinimidamide) $:$ pale yellow solid (42 mg, 51\%); ${ }^{1} \mathbf{H}$ NMR (600 MHz, $\left.\mathbf{C D C l}_{3}\right) \delta 8.56$ (d, $\left.J=7.1 \mathrm{~Hz}, 1 \mathrm{H}\right), 7.80$ $(\mathrm{dd}, J=7.7,7.1 \mathrm{~Hz}, 1 \mathrm{H}), 7.46(\mathrm{dd}, J=7.9,7.7 \mathrm{~Hz}, 1 \mathrm{H}), 7.32(\mathrm{~d}, J=7.9 \mathrm{~Hz}, 1 \mathrm{H})$, $7.08(\mathrm{dd}, J=7.4,7.0 \mathrm{~Hz}, 2 \mathrm{H}), 6.87(\mathrm{t}, J=7.0 \mathrm{~Hz}, 1 \mathrm{H}), 6.68(\mathrm{~d}, J=7.4 \mathrm{~Hz}, 2 \mathrm{H}), 3.48$ (s, 6H); ${ }^{13} \mathbf{C}$ NMR (151 MHz, CDCl 3 ) $\delta$ 161.4, 149.8, 149.5, 147.2, 143.8, 137.3, 130.0, 128.6, 122.5, 122.1, 41.9; IR (neat): $v_{\max }=2982,1568,1273,1125,1024,911,734 \mathrm{~cm}^{-1}$; HRMS $\mathrm{m} / \mathrm{z}$ (EI) calc. for $\mathrm{C}_{14} \mathrm{H}_{15} \mathrm{~N}_{3} \mathrm{OS}\left[\mathrm{M}^{+}\right]$273.0936, found 273.0934; $\mathrm{R}_{f} 0.26$ (DCM/MeOH, 25/1).

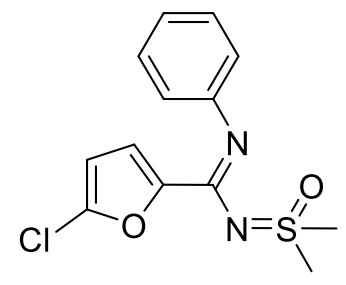

$2 \mathbf{j}$ (5-chloro- $N$-(dimethyl(oxo)- $\lambda^{6}$-sulfanylidene)- $N^{\prime}$-phenylfuran-2-carboximidamide) : white solid (44 mg, 49\%), m.p. 144-148 ${ }^{\circ} \mathrm{C} ;{ }^{1} \mathbf{H}$ NMR (600 MHz, $\left.\mathbf{C D C l}_{3}\right)$ $\delta 7.27(\mathrm{dd}, J=7.7,7.5 \mathrm{~Hz}, 2 \mathrm{H}), 7.04(\mathrm{t}, J=7.5 \mathrm{~Hz}, 1 \mathrm{H}), 6.79$ (d, $J=7.7 \mathrm{~Hz}, 2 \mathrm{H})$, $5.99(\mathrm{~d}, J=3.5 \mathrm{~Hz}, 1 \mathrm{H}), 5.88(\mathrm{~d}, J=3.5 \mathrm{~Hz}, 1 \mathrm{H}), 3.42(\mathrm{~s}, 6 \mathrm{H}) ;{ }^{13} \mathbf{C} \mathbf{N M R}(\mathbf{1 5 1}$ MHz, $\left.\mathbf{C D C l}_{3}\right) \delta 150.6,148.5,146.6,137.8,129.3,123.0,120.6,118.2,108.2$, 42.0; IR (neat): $v_{\max }=3018,2929,1588,1484,1292,1206,1133,762,699 \mathrm{~cm}^{-1}$; HRMS m/z (EI) calc. for $\mathrm{C}_{13} \mathrm{H}_{13} \mathrm{ClN}_{2} \mathrm{O}_{2} \mathrm{~S}\left[\mathrm{M}^{+}\right]$296.0386, found 296.0383; $\mathrm{R}_{f} 0.41$ (hexane/EtOAc, 1/2).

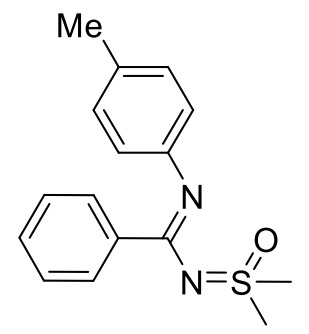

2k ( $N$-(dimethyl(oxo)- $\lambda^{6}$-sulfanylidene)- $N$-( $\left(p\right.$-tolyl)benzimidamide) ${ }^{\mathrm{S} 11}:$ pale yellow solid (43 mg, 32\%); ${ }^{1} \mathbf{H}$ NMR (600 MHz, $\left.\mathbf{C D C l}_{3}\right) \delta 7.33$ (d, J= $\left.7.4 \mathrm{~Hz}, 2 \mathrm{H}\right), 7.23$ (t, $J=7.2 \mathrm{~Hz}, 1 \mathrm{H}), 7.18(\mathrm{dd}, J=7.4,7.2 \mathrm{~Hz}, 2 \mathrm{H}), 6.92(\mathrm{~d}, J=8.0 \mathrm{~Hz}, 2 \mathrm{H}), 6.57(\mathrm{~d}, J=$ $8.0 \mathrm{~Hz}, 2 \mathrm{H}), 3.43$ (s, 6H), 2.23 (s, 3H); ${ }^{13} \mathbf{C ~ N M R ~ ( 1 5 1 ~ M H z , ~ C D C l} 3$ ) $\delta$ 160.5, 147.6, 135.8, 131.5, 129.6, 129.4, 129.1, 127.9, 122.2, 41.8, 21.0; IR (neat): $v_{\max }=3021$, 2925, 1590, 1572, 1446, 1327, 1212, 1127, $649 \mathrm{~cm}^{-1} ; \mathrm{R}_{f} 0.35$ (hexane/EtOAc, 1/2).

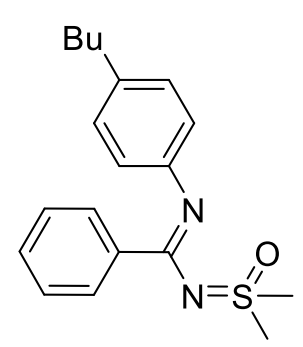

$21 \quad\left(N^{\prime}\right.$-(4-butylphenyl)- $N$-(dimethyl(oxo)- $\lambda^{6}$-sulfanylidene)benzimidamide) colorless liquid (53 mg, 32\%); ${ }^{1} \mathbf{H}$ NMR (600 MHz, $\left.\mathbf{C D C l}_{3}\right) \delta 7.33(\mathrm{~d}, J=7.4 \mathrm{~Hz}$, 2H), $7.22(\mathrm{t}, J=7.2 \mathrm{~Hz}, 1 \mathrm{H}), 7.17(\mathrm{dd}, J=7.4,7.2 \mathrm{~Hz}, 2 \mathrm{H}), 6.92(\mathrm{~d}, J=7.9 \mathrm{~Hz}, 2 \mathrm{H})$, $6.58(\mathrm{~d}, J=7.9 \mathrm{~Hz}, 2 \mathrm{H}), 3.43(\mathrm{~s}, 6 \mathrm{H}), 2.49(\mathrm{t}, J=7.7 \mathrm{~Hz}, 2 \mathrm{H}), 1.52(\mathrm{tt}, J=7.7,7.6$ $\mathrm{Hz}, 2 \mathrm{H}), 1.30(\mathrm{tq}, J=7.6,7.4 \mathrm{~Hz}, 2 \mathrm{H}), 0.89(\mathrm{t}, J=7.4 \mathrm{~Hz}, 3 \mathrm{H}) ;{ }^{13} \mathbf{C}$ NMR (151 MHz, $\left.\mathbf{C D C l}_{3}\right) \delta 160.4,147.8,136.7,135.8,129.7,129.1,128.7,127.8,122.1,41.8$, 35.2, 33.9, 22.4, 14.2; IR (neat): $v_{\max }=2962,2254,1592,1216,903,723 \mathrm{~cm}^{-1}$; HRMS m/z (EI) calc. for $\mathrm{C}_{19} \mathrm{H}_{24} \mathrm{~N}_{2} \mathrm{OS}\left[\mathrm{M}^{+}\right]$328.1609, found 328.1609; $\mathrm{R}_{f} 0.39$ (hexane/EtOAc, 1/2). 


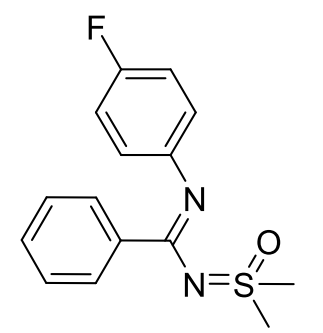

$2 \mathbf{m}$ ( $N$-(dimethyl(oxo)- $\lambda^{6}$-sulfanylidene)- $N^{\prime}$-(4-fluorophenyl)benzimidamide) : pale yellow solid (74 mg, 51\%), m.p. 146-150 ${ }^{\circ} \mathrm{C} ;{ }^{1} \mathbf{H}$ NMR (600 MHz, $\left.\mathbf{C D C l}_{3}\right) \delta 7.29(\mathrm{~d}$, $J=7.1 \mathrm{~Hz}, 2 \mathrm{H}), 7.24(\mathrm{t}, J=7.4 \mathrm{~Hz}, 1 \mathrm{H}), 7.19(\mathrm{dd}, J=7.4,7.1 \mathrm{~Hz}, 2 \mathrm{H}), 6.80(\mathrm{dd}, J=$ $\left.8.7 \mathrm{~Hz},{ }^{3} J_{H-F}=8.7 \mathrm{~Hz}, 2 \mathrm{H}\right), 6.60\left(\mathrm{dd}, J=8.7 \mathrm{~Hz},{ }^{4} J_{H-F}=4.9 \mathrm{~Hz}, 2 \mathrm{H}\right), 3.44(\mathrm{~s}, 6 \mathrm{H})$; ${ }^{13}$ C NMR (151 MHz, CDCl $) \delta 161.4,158.8\left(\mathrm{~d},{ }^{1} J_{C-F}=240.1 \mathrm{~Hz}\right), 146.4\left(\mathrm{~d},{ }^{4} J_{C-F}=\right.$ $2.4 \mathrm{~Hz}), 135.6,129.5,129.3,128.0,123.4\left(\mathrm{~d},{ }^{3} J_{C-F}=7.5 \mathrm{~Hz}\right), 115.4\left(\mathrm{~d},{ }^{2} J_{C-F}=21.1 \mathrm{~Hz}\right), 42.0$; IR (neat): $v_{\max }=2987,1592,1573,1500,1382,1210,1128,1024,650 \mathrm{~cm}^{-1} ;$ HRMS m/z (EI) calc. for $\mathrm{C}_{15} \mathrm{H}_{15} \mathrm{FN}_{2} \mathrm{OS}$ $\left[\mathrm{M}^{+}\right]$290.0889, found 290.0890; $\mathrm{R}_{f} 0.30$ (hexane/EtOAc, 1/2).

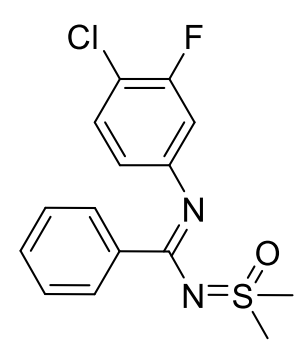

2n ( $N$-(4-chloro-3-fluorophenyl)- $N$-(dimethyl(oxo)- $\lambda^{6}$-sulfanylidene)benzimidamide) : colorless liquid (81 mg, 50\%); ${ }^{1} \mathbf{H}$ NMR (600 MHz, $\left.\mathbf{C D C l}_{3}\right) \delta 7.31-7.22(\mathrm{~m}$, $5 \mathrm{H}), 7.08(\mathrm{t}, J=7.6 \mathrm{~Hz}, 1 \mathrm{H}), 6.49\left(\mathrm{~d},{ }^{3} J_{H-F}=9.0 \mathrm{~Hz}, 1 \mathrm{H}\right), 6.37(\mathrm{~d}, J=6.0 \mathrm{~Hz}, 1 \mathrm{H})$, $3.43(\mathrm{~s}, 6 \mathrm{H}) ;{ }^{13} \mathbf{C}$ NMR (151 MHz, $\left.\mathbf{C D C l}_{3}\right) \delta 162.0,158.2\left(\mathrm{~d},{ }^{1} J_{C-F}=247.8 \mathrm{~Hz}\right)$, $150.7\left(\mathrm{~d},{ }^{3} J_{C-F}=8.4 \mathrm{~Hz}\right), 135.1\left(\mathrm{~d},{ }^{3} J_{C-F}=8.8 \mathrm{~Hz}\right), 130.2,129.7,129.4,128.2,119.1$ $\left(\mathrm{d},{ }^{4} J_{C-F}=3.0 \mathrm{~Hz}\right), 113.9\left(\mathrm{~d},{ }^{2} J_{C-F}=28.9 \mathrm{~Hz}\right), 110.8\left(\mathrm{~d},{ }^{2} J_{C-F}=21.7 \mathrm{~Hz}\right), 42.0 ; \mathbf{I R}$ (neat): $v_{\max }=2928,2350,1568,1478,1331,1213,1117,1024,909,730 \mathrm{~cm}^{-1} ; \mathrm{HRMS} \mathrm{m} / \mathrm{z}$ (EI) calc. for $\mathrm{C}_{15} \mathrm{H}_{14} \mathrm{ClFN}_{2} \mathrm{OS}\left[\mathrm{M}^{+}\right]$324.0499, found 324.0499; $\mathrm{R}_{f} 0.37$ (hexane/EtOAc, 1/2).

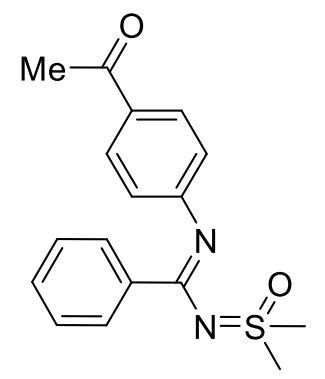

20 ( $N^{\prime}$-(4-acetylphenyl)- $N$-(dimethyl(oxo)- $\lambda^{6}$-sulfanylidene)benzimidamide) : white solid (97 mg, 62\%), m.p. 146-150 ${ }^{\circ} \mathrm{C} ;{ }^{1} \mathbf{H}$ NMR (600 MHz, $\left.\mathbf{C D C l}_{3}\right) \delta 7.76(\mathrm{~d}, J=$ $8.3 \mathrm{~Hz}, 2 \mathrm{H}), 7.43-7.32(\mathrm{~m}, 2 \mathrm{H}), 7.27$ (t, $J=7.3 \mathrm{~Hz}, 1 \mathrm{H}), 7.20$ (dd, $J=7.5,7.3 \mathrm{~Hz}$, 2H), $6.74(\mathrm{~d}, J=7.5 \mathrm{~Hz}, 2 \mathrm{H}), 3.41(\mathrm{~s}, 6 \mathrm{H}), 2.51$ (s, 3H); ${ }^{13} \mathrm{C}$ NMR (151 MHz, $\left.\mathbf{C D C l}_{3}\right) \delta 197.5,155.2,131.5,129.8,129.7,129.6,129.5,128.2,128.1,122.3,42.1$, 26.5; IR (neat): $v_{\max }=3024,2930,1670,1584,1330,1271,1126,649 \mathrm{~cm}^{-1}$; HRMS $\mathrm{m} / \mathrm{z}$ (EI) calc. for $\mathrm{C}_{17} \mathrm{H}_{18} \mathrm{~N}_{2} \mathrm{O}_{2} \mathrm{~S}\left[\mathrm{M}^{+}\right]$314.1089, found 314.1091; $\mathrm{R}_{f} 0.23$ (hexane/EtOAc, 1/2).

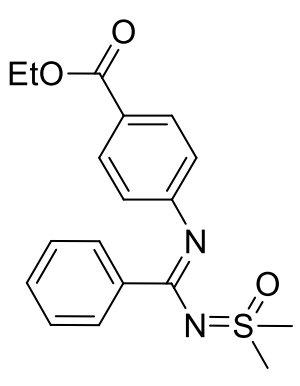

2p (ethyl 4-((((dimethyl(oxo)- $\lambda^{6}$-sulfanylidene)amino)(phenyl)methylene)amino)benzoate) : light yellow liquid (52 mg, 50\%); ${ }^{1} \mathbf{H}$ NMR (300 $\left.\mathbf{M H z}, \mathbf{C D C l}_{3}\right) \delta 7.83$ $(\mathrm{d}, J=8.4 \mathrm{~Hz}, 2 \mathrm{H}), 7.42-7.25(\mathrm{~m}, 3 \mathrm{H}), 7.21(\mathrm{dd}, J=7.6,7.3 \mathrm{~Hz}, 2 \mathrm{H}), 6.72(\mathrm{~d}, J=$ $7.6 \mathrm{~Hz}, 2 \mathrm{H}), 4.31(\mathrm{q}, J=7.1 \mathrm{~Hz}, 2 \mathrm{H}), 3.42(\mathrm{~s}, 6 \mathrm{H}), 1.36(\mathrm{t}, J=7.1 \mathrm{~Hz}, 3 \mathrm{H}) ;{ }^{13} \mathrm{C}$ NMR (151 MHz, $\left.\mathbf{C D C l}_{3}\right) \delta 166.9,161.2,154.9,130.6,129.7,129.6,128.2,128.1$, 124.2, 122.1, 60.7, 42.0, 14.6; IR (neat): $v_{\max }=2970,1739,1585,1567,1366,1273$, 
1216, 1125, $702 \mathrm{~cm}^{-1}$; HRMS m/z (EI) calc. for $\mathrm{C}_{18} \mathrm{H}_{20} \mathrm{~N}_{2} \mathrm{O}_{3} \mathrm{~S}\left[\mathrm{M}^{+}\right]$344.1195, found 344.1195; $\mathrm{R}_{f} 0.35$ (hexane/EtOAc, 1/2).

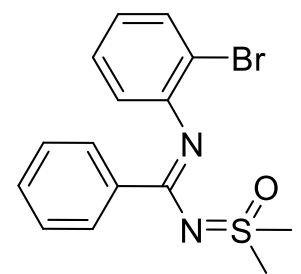

2q ( $N^{\prime}$-(2-bromophenyl)- $N$-(dimethyl(oxo)- $\lambda^{6}$-sulfanylidene)benzimidamide) : pale yellow solid (84 mg, 48\%), m.p. 155-160 ${ }^{\circ} \mathrm{C} ;{ }^{1} \mathbf{H}$ NMR (600 MHz, $\left.\mathbf{C D C l}_{3}\right) \delta 7.49(\mathrm{~d}$, $J=7.7 \mathrm{~Hz}, 1 \mathrm{H}), 7.33(\mathrm{~d}, J=6.1 \mathrm{~Hz}, 2 \mathrm{H}), 7.24(\mathrm{t}, J=6.8 \mathrm{~Hz}, 1 \mathrm{H}), 7.19(\mathrm{dd}, J=6.8$, $6.1 \mathrm{~Hz}, 2 \mathrm{H}), 6.96(\mathrm{dd}, J=7.1,6.6 \mathrm{~Hz}, 1 \mathrm{H}), 6.76(\mathrm{dd}, J=7.7,7.1 \mathrm{~Hz}, 1 \mathrm{H}), 6.46(\mathrm{~d}, J$ $=6.6 \mathrm{~Hz}, 1 \mathrm{H}), 3.52(\mathrm{~s}, 6 \mathrm{H}) ;{ }^{13} \mathbf{C} \mathbf{N M R}\left(\mathbf{1 5 1} \mathbf{~ M H z}, \mathbf{C D C l}_{3}\right) \delta$ 161.6, 149.1, 135.7,

132.5, 132.4, 129.5, 129.1, 128.0, 127.9, 123.5, 117.7, 42.3; IR (neat): $v_{\max }=2965,1593,1574,1329$, 1296, 1134, 1028, 649, $465 \mathrm{~cm}^{-1}$; HRMS m/z (EI) calc. for $\mathrm{C}_{15} \mathrm{H}_{15} \mathrm{BrN}_{2} \mathrm{OS}\left[\mathrm{M}^{+}\right]$350.0088, found $350.0085 ; \mathrm{R}_{f} 0.58$ (hexane/EtOAc, $1 / 2$ ).

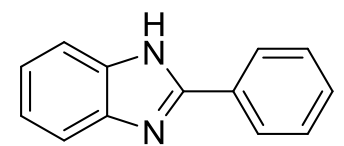

3a (2-phenyl-1H-benzo[ $d]$ imidazole $)^{\mathrm{S} 12}$ : yellow solid $(86 \mathrm{mg}, 89 \%) ;{ }^{1} \mathbf{H}$ NMR (600 MHz, DMSO-d $)_{6} \delta 8.18(\mathrm{~d}, J=7.9 \mathrm{~Hz}, 2 \mathrm{H}), 7.62-7.60(\mathrm{~m}, 2 \mathrm{H}), 7.56(\mathrm{dd}, J$ = 7.9, 7.3 Hz, 2H), $7.50(\mathrm{t}, J=7.3 \mathrm{~Hz}, 1 \mathrm{H}), 7.23-7.20(\mathrm{~m}, 2 \mathrm{H}) ;{ }^{13} \mathbf{C}$ NMR $(\mathbf{1 5 1}$ MHz, DMSO- $\left.\boldsymbol{d}_{\boldsymbol{6}}\right) \delta 151.3,139.3,130.1,130.0,129.1,126.6,122.4,115.1$; IR (neat): $v_{\max }=3439,1663$, $1444,1025,622 \mathrm{~cm}^{-1} ; \mathrm{R}_{f} 0.71$ (hexane/EtOAc, 1/2).

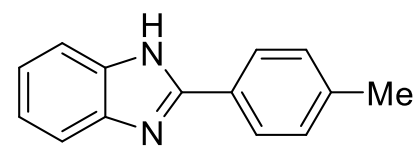

3b (2-(p-tolyl)-1H-benzo[d]imidazole) ${ }^{\mathrm{S} 13}$ : white solid $(78 \mathrm{mg}, 75 \%) ;{ }^{1} \mathbf{H}$ NMR (600 MHz, DMSO-d $\left.{ }_{6}\right) \delta 8.07(\mathrm{~d}, J=8.0 \mathrm{~Hz}, 2 \mathrm{H}), 7.60-7.54(\mathrm{~m}, 2 \mathrm{H})$, $7.35(\mathrm{~d}, J=8.0 \mathrm{~Hz}, 2 \mathrm{H}), 7.20-7.17(\mathrm{~m}, 2 \mathrm{H}), 2.38(\mathrm{~s}, 3 \mathrm{H}) ;{ }^{13} \mathbf{C}$ NMR (151 MHz, DMSO- $\boldsymbol{d}_{\boldsymbol{\sigma}}$ ) $\delta 151.4,139.6,129.6,129.6,127.5,127.5,126.4,122.0,21.0$; IR (neat): $v_{\max }=3447$, $3071,1636,1296,1025,623 \mathrm{~cm}^{-1} ; \mathrm{R}_{f} 0.73$ (hexane/EtOAc, 1/2).

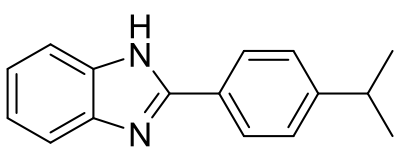

3c (2-(4-isopropylphenyl)- $1 H$-benzo $[d]$ imidazole $)^{\mathrm{S} 12}$ : white solid $(85 \mathrm{mg}$, $72 \%) ;{ }^{1} \mathbf{H}$ NMR (600 MHz, DMSO-d $\left.)\right) \delta 12.83(\mathrm{bs}, 1 \mathrm{H}), 8.10(\mathrm{~d}, J=8.2 \mathrm{~Hz}$, $2 \mathrm{H}), 7.70-7.47(\mathrm{~m}, 2 \mathrm{H}), 7.42(\mathrm{~d}, J=8.2 \mathrm{~Hz}, 2 \mathrm{H}), 7.20-7.17(\mathrm{~m}, 2 \mathrm{H}), 2.96$ (sept, $J=6.9 \mathrm{~Hz}, 1 \mathrm{H}), 1.25(\mathrm{~d}, J=6.9 \mathrm{~Hz}, 6 \mathrm{H}) ;{ }^{13} \mathrm{C}$ NMR (151 MHz, DMSO-d 6 ) $\delta 151.4,150.4,127.9$, $126.9,126.5,121.8,118.6,111.3,33.4,23.7$; IR (neat): $v_{\max }=3448,2249,2124,1622,1025,820,758$ $\mathrm{cm}^{-1} ; \mathrm{R}_{f} 0.81$ (hexane/EtOAc, $1 / 2$ ). 


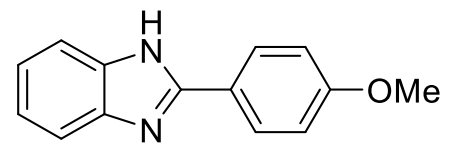

3d (2-(4-methoxyphenyl)- $1 H$-benzo[ $d]$ imidazole) ${ }^{\mathrm{S} 12}$ : pale yellow solid (76 $\mathrm{mg}, 68 \%) ;{ }^{1} \mathbf{H}$ NMR (600 MHz, DMSO-d 6 ) $\delta 8.13(\mathrm{~d}, J=8.8 \mathrm{~Hz}, 2 \mathrm{H})$, $7.60-7.52(\mathrm{~m}, 2 \mathrm{H}), 7.18-7.15(\mathrm{~m}, 2 \mathrm{H}), 7.11(\mathrm{~d}, J=8.8 \mathrm{~Hz}, 2 \mathrm{H}), 3.83(\mathrm{~s}$, 3H); ${ }^{13}$ C NMR (151 MHz, DMSO-d $\left.{ }_{6}\right) \delta$ 160.7, 151.4, 128.1, 122.8, 121.8, 121.8, 114.4, 112.0, 55.4.; IR (neat): $v_{\max }=3449,1613,1433,1251,1025,623 \mathrm{~cm}^{-1} ; \mathrm{R}_{f} 0.50$ (hexane/EtOAc, $1 / 2$ ).

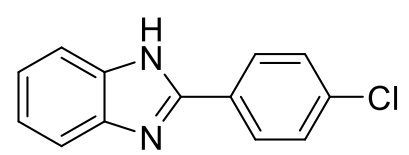

3e (2-(4-chlorophenyl)- $1 H$-benzo $[d]$ imidazole $)^{\mathrm{S} 13}$ : colorless solid (62 mg, 54\%); ${ }^{1} \mathrm{H}$ NMR (600 MHz, DMSO-d $) \delta 8.18(\mathrm{~d}, J=8.7 \mathrm{~Hz}, 2 \mathrm{H}), 7.63(\mathrm{~d}, J$ $=8.7 \mathrm{~Hz}, 2 \mathrm{H}), 7.60-7.58(\mathrm{~m}, 2 \mathrm{H}), 7.23-7.20(\mathrm{~m}, 2 \mathrm{H}) ;{ }^{13} \mathbf{C} \mathbf{N M R}(\mathbf{1 5 1}$ MHz, DMSO-d $\left.\boldsymbol{d}_{6}\right) \delta 150.2,139.5,134.6,129.1,129.1,128.2,122.4,122.4$; IR (neat): $v_{\max }=3449,1621$, $1430,1025,758,622 \mathrm{~cm}^{-1} ; \mathrm{R}_{f} 0.73$ (hexane/EtOAc, 1/2).

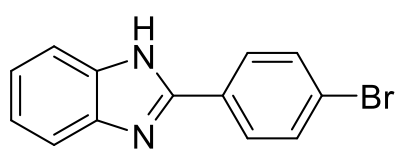

3f (2-(4-bromophenyl)-1H-benzo[ $[d]$ imidazole) $)^{\mathrm{S} 12}$ : white solid (79 $\mathrm{mg}, 58 \%$; ${ }^{1}$ H NMR (600 MHz, DMSO-d $\left.)\right) \delta 8.12(\mathrm{~d}, J=8.5 \mathrm{~Hz}, 2 \mathrm{H}), 7.76(\mathrm{~d}, J=8.5$ $\mathrm{Hz}, 2 \mathrm{H}), 7.62-7.58(\mathrm{~m}, 2 \mathrm{H}), 7.23-7.20(\mathrm{~m}, 2 \mathrm{H}) ;{ }^{13} \mathrm{C}$ NMR (151 MHz, DMSO-d $\boldsymbol{d}_{\boldsymbol{6}} \delta 150.3,132.0,129.5,128.4,128.4,123.3,122.4,122.3$; IR (neat): $v_{\max }=3445,2250,2124$, $1620,1025,820,758 \mathrm{~cm}^{-1} ; \mathrm{R}_{f} 0.74$ (hexane/EtOAc, 1/2).

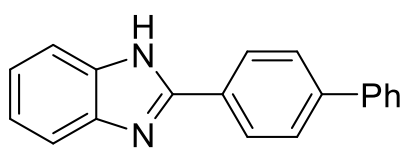

$3 g$ (2-([1,1'-biphenyl]-4-yl)-1H-benzo[d]imidazole) $)^{\mathrm{S} 14}$ : white solid (88 mg, 65\%); ${ }^{1}$ H NMR (600 MHz, DMSO-d $\left.d_{6}\right) 12.96$ (bs, $\left.1 \mathrm{H}\right), 8.27$ (d, $J=8.5 \mathrm{~Hz}$, 2H), $7.88(\mathrm{~d}, J=8.5 \mathrm{~Hz}, 2 \mathrm{H}), 7.78(\mathrm{~d}, J=8.0 \mathrm{~Hz}, 2 \mathrm{H}), 7.68(\mathrm{~d}, J=7.0 \mathrm{~Hz}$, $1 \mathrm{H}), 7.55(\mathrm{~d}, J=7.2 \mathrm{~Hz}, 1 \mathrm{H}), 7.51(\mathrm{dd}, J=8.0,7.5 \mathrm{~Hz}, 2 \mathrm{H}), 7.41$ (t, $J=7.5 \mathrm{~Hz}, 1 \mathrm{H}), 7.23(\mathrm{dd}, J=8.2$, $7.0 \mathrm{~Hz}, 1 \mathrm{H}), 7.20(\mathrm{dd}, J=8.2,7.2 \mathrm{~Hz}, 1 \mathrm{H}) ;{ }^{13} \mathbf{C}$ NMR (151 MHz, DMSO-d 6 ) $\delta$ 150.9, 141.3, 139.3, 129.2, 129.1, 127.9, 127.2, 127.0, 126.7, 121.7, 118.9, 111.3; IR (neat): $v_{\max }=3445,2250,1622,1025,820,758$ $\mathrm{cm}^{-1} ; \mathrm{R}_{f} 0.72$ (hexane/EtOAc, 1/2).

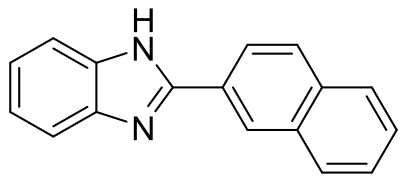

3h (2-(naphthalen-2-yl)-1H-benzo[d]imidazole) $)^{\mathrm{S} 13}$ : white solid $(56 \mathrm{mg}$, 46\%); ${ }^{1} \mathrm{H}$ NMR (600 MHz, DMSO-d $\left.{ }_{6}\right) \delta 13.07$ (bs, 1H), $8.74(\mathrm{~s}, 1 \mathrm{H}), 8.32$ $(\mathrm{d}, J=8.6 \mathrm{~Hz}, 1 \mathrm{H}), 8.08(\mathrm{~d}, J=8.6 \mathrm{~Hz}, 1 \mathrm{H}), 8.05(\mathrm{~d}, J=8.5 \mathrm{~Hz}, 1 \mathrm{H}), 7.99(\mathrm{~d}$, $J=8.5 \mathrm{~Hz}, 1 \mathrm{H}), 7.70(\mathrm{~d}, J=7.1 \mathrm{~Hz}, 1 \mathrm{H}), 7.62-7.58(\mathrm{~m}, 2 \mathrm{H}), 7.57(\mathrm{~d}, J=7.4 \mathrm{~Hz}, 1 \mathrm{H}), 7.25(\mathrm{dd}, J=7.9$, $7.1 \mathrm{~Hz}, 1 \mathrm{H}), 7.21(\mathrm{dd}, J=7.9,7.4 \mathrm{~Hz}, 1 \mathrm{H}) ;{ }^{13} \mathbf{C}$ NMR (151 MHz, DMSO-d 6 ) $\delta$ 151.3, 144.0, 133.5, 132.8, 128.6, 128.5, 127.8, 127.6, 127.1, 126.9, 125.8, 124.0, 118.9, 111.4; IR (neat): $v_{\max }=3448,2250,1620$, $1025,820,758 \mathrm{~cm}^{-1} ; \mathrm{R}_{f} 0.70$ (hexane/EtOAc, 1/2). 


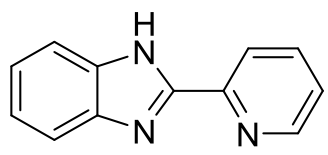

3i (2-(pyridin-2-yl)-1H-benzo[ $[d]$ imidazole $)^{\mathrm{S} 14}$ : white solid $(31 \mathrm{mg}, 53 \%) ;{ }^{1} \mathbf{H}$ NMR (600 MHz, DMSO-d 6 ) $\delta 13.09$ (bs, 1H), 8.73 (d, $J=4.7 \mathrm{~Hz}, 1 \mathrm{H}), 8.33$ (d, $J$ $=7.8 \mathrm{~Hz}, 1 \mathrm{H}), 7.99(\mathrm{dd}, J=7.8,7.5 \mathrm{~Hz}, 1 \mathrm{H}), 7.73-7.65(\mathrm{~m}, 1 \mathrm{H}), 7.61-7.53(\mathrm{~m}$, 1H), $7.51(\mathrm{dd}, J=7.5,4.7 \mathrm{~Hz}, 1 \mathrm{H}), 7.27-7.19(\mathrm{~m}, 2 \mathrm{H}) ;{ }^{13}$ C NMR (151 MHz, DMSO-d $)$ ) $\delta$ 150.8, 149.4, 148.6, 143.9, 137.6, 124.8, 123.2, 121.5, 112.1; IR (neat): $v_{\max }=3467,2984,1735,1373,1236,1043$, $608 \mathrm{~cm}^{-1} ; \mathrm{R}_{f} 0.30$ (hexane/EtOAc, 1/2).

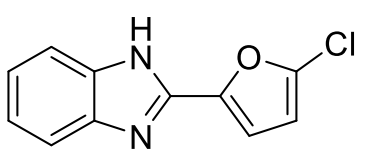

3j (2-(5-chlorofuran-2-yl)-1H-benzo[d]imidazole) ${ }^{\mathrm{S} 15}$ : pale yellow solid (30 mg, 45\%); ${ }^{1}$ H NMR (600 MHz, DMSO-d $) \delta 7.59-7.53(\mathrm{~m}, 2 \mathrm{H}), 7.26(\mathrm{~d}, J=3.5$ $\mathrm{Hz}, 1 \mathrm{H}), 7.22-7.20(\mathrm{~m}, 2 \mathrm{H}), 6.76(\mathrm{~d}, J=3.5 \mathrm{~Hz}, 1 \mathrm{H}) ;{ }^{13} \mathrm{C}$ NMR (151 MHz, DMSO- $\left.\boldsymbol{d}_{\boldsymbol{6}}\right) \delta 145.4,143.9,142.5,136.9,136.5,122.5,112.7,109.6$; IR (neat): $v_{\max }=3445,1621,1225$, $1025,680,622 \mathrm{~cm}^{-1} ; \mathrm{R}_{f} 0.58$ (hexane/EtOAc, 1/2).

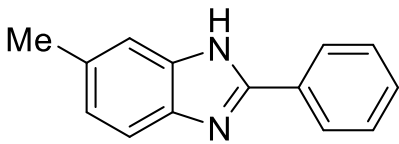

3k (6-methyl-2-phenyl-1H-benzo[d]imidazole) $)^{\mathrm{S} 13}$ : pale yellow solid (102 mg, 98\%); ${ }^{1} \mathbf{H}$ NMR (600 MHz, DMSO-d $\left.\boldsymbol{d}_{\boldsymbol{6}}\right) \delta .15$ (d, $\left.J=7.8 \mathrm{~Hz}, 2 \mathrm{H}\right), 7.53$ $(\mathrm{dd}, J=7.8,7.3 \mathrm{~Hz}, 2 \mathrm{H}), 7.50-7.48(\mathrm{~m}, 1 \mathrm{H}), 7.47(\mathrm{t}, J=7.3 \mathrm{~Hz}, 1 \mathrm{H}), 7.41$ - $7.36(\mathrm{~m}, 1 \mathrm{H}), 7.02(\mathrm{~d}, J=8.1 \mathrm{~Hz}, 1 \mathrm{H}), 2.41(\mathrm{~s}, 3 \mathrm{H}) ;{ }^{13} \mathbf{C}$ NMR (151 MHz, DMSO-d $\left.)\right) \delta$ 151.2, 131.7, $130.4,130.0,129.2,127.6,127.6,127.6,127.4,126.6,124.0,21.6$; IR (neat): $v_{\max }=3436,1660,1451$, $1024,622 \mathrm{~cm}^{-1} ; \mathrm{R}_{f} 0.69$ (hexane/EtOAc, 1/2).

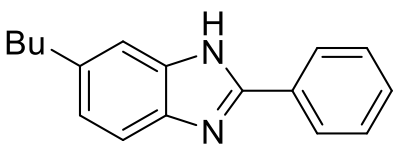

31 (6-butyl-2-phenyl-1 $H$-benzo $[d]$ imidazole $)^{\mathrm{S} 16}$ : white solid $(120 \mathrm{mg}, 96 \%)$; ${ }^{1}$ H NMR (600 MHz, DMSO-d $\left.\boldsymbol{d}_{6}\right) \delta 8.17(\mathrm{~d}, J=7.6 \mathrm{~Hz}, 2 \mathrm{H}), 7.53(\mathrm{dd}, J=7.6$, $7.3 \mathrm{~Hz}, 2 \mathrm{H}), 7.51-7.45(\mathrm{~m}, 1 \mathrm{H}), 7.47(\mathrm{t}, J=7.3 \mathrm{~Hz}, 1 \mathrm{H}), 7.41-7.34(\mathrm{~m}$, $1 \mathrm{H}), 7.03(\mathrm{~d}, J=8.1 \mathrm{~Hz}, 1 \mathrm{H}), 2.68(\mathrm{t}, J=7.5 \mathrm{~Hz}, 2 \mathrm{H}), 1.60(\mathrm{tt}, J=7.5,7.4 \mathrm{~Hz}, 2 \mathrm{H}), 1.32(\mathrm{tq}, J=7.4,7.3$ $\mathrm{Hz}, 2 \mathrm{H}), 0.90$ (t, $J=7.3 \mathrm{~Hz}, 3 \mathrm{H}) ;{ }^{13} \mathbf{C}$ NMR (151 MHz, DMSO-d $) \delta 151.0,136.6,131.5,130.4,129.7$, $128.9,128.9,126.4,123.0,118.0,110.6,35.2,33.9,21.8,13.9$; IR (neat): $v_{\max }=3450,2250,2124,1624$, $1025,820,758 \mathrm{~cm}^{-1} ; \mathrm{R}_{f} 0.81$ (hexane/EtOAc, 1/2).

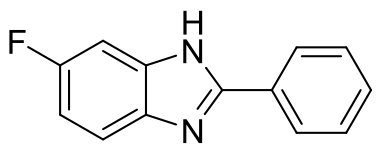

3m (6-fluoro-2-phenyl-1H-benzo $[d]$ imidazole $)^{\mathrm{S} 13}$ : white solid (72 $\left.\mathrm{mg}, 68 \%\right)$; ${ }^{1}$ H NMR (600 MHz, DMSO-d $\left.{ }_{6}\right) \delta 8.16(\mathrm{~d}, J=7.2 \mathrm{~Hz}, 2 \mathrm{H}), 7.59(\mathrm{dd}, J=8.7$ $\left.\mathrm{Hz},{ }^{4} J_{H-F}=4.9 \mathrm{~Hz}, 1 \mathrm{H}\right), 7.55(\mathrm{dd}, J=7.5,7.2 \mathrm{~Hz}, 2 \mathrm{H}), 7.50(\mathrm{t}, J=7.5 \mathrm{~Hz}, 1 \mathrm{H})$, $7.39\left(\mathrm{~d},{ }^{3} J_{H-F}=9.3 \mathrm{~Hz}, 1 \mathrm{H}\right), 7.06\left(\mathrm{dd},{ }^{3} J_{H-F}=9.8 \mathrm{~Hz}, J=8.7 \mathrm{~Hz}, 1 \mathrm{H}\right) ;{ }^{13} \mathbf{C}$ NMR (151 MHz, DMSO-d 6 ) $\delta$ $158.7\left({ }^{1} J_{C-F}=235.6 \mathrm{~Hz}\right), 157.9,152.7,136.8,130.1,129.9,129.0,127.6,126.5,114.9\left({ }^{2} J_{C-F}=31.7 \mathrm{~Hz}\right)$, 
$110.2\left({ }^{2} J_{C-F}=25.7 \mathrm{~Hz}\right) ;$ IR (neat): $v_{\max }=3481,1617,1457,1296,1025,622 \mathrm{~cm}^{-1} ; \mathrm{R}_{f} 0.74$ (hexane/EtOAc, 1/2).

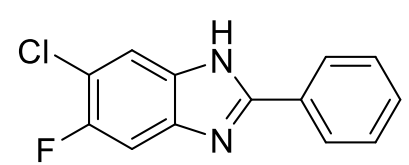

3n (6-chloro-5-fluoro-2-phenyl-1H-benzo $[d]$ imidazole $)^{\mathrm{S} 17}:$ colorless liquid (62 mg, 50\%); ${ }^{1} \mathbf{H}$ NMR (600 MHz, DMSO-d $) \delta 8.18(\mathrm{~d}, J=7.0 \mathrm{~Hz}, 2 \mathrm{H})$, $7.81\left(\mathrm{~d},{ }^{4} J_{H-F}=6.8 \mathrm{~Hz}, 1 \mathrm{H}\right), 7.66\left(\mathrm{~d},{ }^{3} J_{H-F}=9.5 \mathrm{~Hz}, 1 \mathrm{H}\right), 7.58-7.55(\mathrm{~m}, 3 \mathrm{H})$; ${ }^{13}$ C NMR (151 MHz, DMSO-d $\left.\boldsymbol{d}_{6}\right) \delta 153.7\left({ }^{1} J_{C-F}=240.1 \mathrm{~Hz}\right), 153.3,130.7,130.6,129.1,129.1,128.7$, $126.9,126.8,123.4,114.6\left({ }^{2} J_{C-F}=21.1 \mathrm{~Hz}\right)$; IR (neat): $v_{\max }=3417,2970,1738,1658,1366,1023,1001$, $760,613,527 \mathrm{~cm}^{-1} ; \mathrm{R}_{f} 0.80$ (hexane/EtOAc, 1/2).<smiles>CC(=O)c1ccc2nc(-c3ccccc3)[nH]c2c1</smiles>

30 (1-(2-phenyl-1H-benzo[ $d]$ imidazol-6-yl)ethan-1-one) ${ }^{\mathrm{S} 18}$ : white solid $(66 \mathrm{mg}, 56 \%) ;{ }^{1} \mathbf{H}$ NMR (600 MHz, $\left.\mathbf{C D C l}_{3}\right) \delta 8.26(\mathrm{~d}, J=1.4 \mathrm{~Hz}, 1 \mathrm{H})$, $8.17(\mathrm{dd}, J=7.8,1.3 \mathrm{~Hz}, 2 \mathrm{H}), 7.90(\mathrm{dd}, J=8.5,1.4 \mathrm{~Hz}, 1 \mathrm{H}), 7.64(\mathrm{~d}, J=$ $8.5 \mathrm{~Hz}, 1 \mathrm{H}), 7.45$ - 7.39 (m, 3H), 2.61 (s, 3H); ${ }^{13} \mathbf{C}$ NMR (151 MHz, $\left.\mathbf{C D C l}_{3}\right) \delta$ 198.3, 154.4, 142.1, 138.2, 132.9, 131.5, 129.5, 128.5, 127.4, 124.2, 116.3, 115.2, 27.0; IR (neat): $v_{\max }=2970,1738,1674,1366$, $1216,695 \mathrm{~cm}^{-1} ; \mathrm{R}_{f} 0.49$ (hexane/EtOAc, 1/2).<smiles>CCOC(=O)c1ccc2nc(-c3ccccc3)[nH]c2c1</smiles>

3p (ethyl 2-phenyl-1H-benzo[ $\left[\right.$ ]imidazole-6-carboxylate) ${ }^{\mathrm{S} 16}$ : white solid (40 mg, 50\%); ${ }^{1} \mathbf{H}$ NMR (600 MHz, $\left.\mathbf{C D C l}_{3}\right) 8.42-8.21(\mathrm{~m}, 1 \mathrm{H}), 8.15-$ $8.06(\mathrm{~m}, 2 \mathrm{H}), 7.99(\mathrm{dd}, J=8.5,1.3 \mathrm{~Hz}, 1 \mathrm{H}), 7.92-7.65(\mathrm{~m}, 1 \mathrm{H}), 7.50-$ $7.43(\mathrm{~m}, 3 \mathrm{H}), 4.40(\mathrm{q}, J=7.0 \mathrm{~Hz}, 2 \mathrm{H}), 1.41(\mathrm{t}, J=7.0 \mathrm{~Hz}, 3 \mathrm{H}) ;{ }^{13} \mathbf{C}$ NMR (151 MHz, CDCl$) \delta 167.5$, $156.5,130.9,130.9,129.5,129.4,129.3,127.1,125.4,110.2,110.1,97.4,61.3,14.6$; IR (neat): $v_{\max }=$ 2987, 1705, 1475, 1381, 1301, 1096, $650 \mathrm{~cm}^{-1} ; \mathrm{R}_{f} 0.72$ (hexane/EtOAc, 1/2).

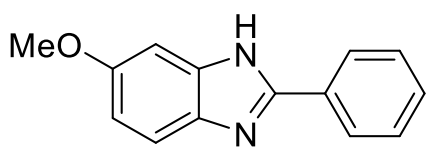

3r (6-methoxy-2-phenyl-1H-benzo[ $d]$ imidazole $)^{\mathrm{S} 13}$ : pale yellow solid (108 mg, 96\%); ${ }^{1}$ H NMR (600 MHz, DMSO-d $) \delta 8.14(\mathrm{~d}, J=7.3 \mathrm{~Hz}, 2 \mathrm{H})$, $7.53(\mathrm{dd}, J=7.6,7.3 \mathrm{~Hz}, 2 \mathrm{H}), 7.49(\mathrm{~d}, J=8.4 \mathrm{~Hz}, 1 \mathrm{H}), 7.46(\mathrm{t}, J=7.6 \mathrm{~Hz}$, 1H), $7.15-7.03(\mathrm{~m}, 1 \mathrm{H}), 6.84(\mathrm{dd}, J=8.4,2.4 \mathrm{~Hz}, 1 \mathrm{H}), 3.80(\mathrm{~s}, 3 \mathrm{H}) ;{ }^{13} \mathbf{C}$ NMR (151 MHz, DMSO-d 6 ) $\delta$ $155.9,150.9,136.3,130.4,129.5,129.4,129.1,128.9,126.2,119.1,111.7,55.5$; IR (neat): $v_{\max }=3441$, $1664,1463,1025,623 \mathrm{~cm}^{-1} ; \mathrm{R}_{f} 0.59$ (hexane/EtOAc, 1/2). 


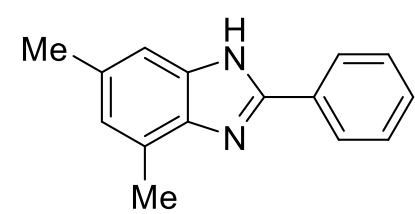

3s (4,6-dimethyl-2-phenyl-1H-benzo[ $[d \text { imidazole })^{\mathrm{S} 13}$ : white solid (76 mg, 68\%); ${ }^{1}$ H NMR (600 MHz, DMSO-d $) \delta 12.69$ (bs, 1H), 8.23 - 8.14 (m, 2H), $7.53(\mathrm{dd}, J=7.4,7.3 \mathrm{~Hz}, 2 \mathrm{H}), 7.46(\mathrm{t}, J=7.3 \mathrm{~Hz}, 1 \mathrm{H}), 7.15(\mathrm{~s}, 1 \mathrm{H})$, $6.82(\mathrm{~s}, 1 \mathrm{H}), 2.53$ (s, 3H), 2.38 (s, 3H); ${ }^{13} \mathrm{C}$ NMR (151 MHz, DMSO-d 6 ) $\delta$ 153.0, 134.9, 132.9, 130.6, 129.6, 128.9, 126.4, 126.1, 124.7, 123.8, 108.6, 21.4, 16.9; IR (neat): $v_{\max }=$ $3441,1624,1454,1025,820,758 \mathrm{~cm}^{-1} ; \mathrm{R}_{f} 0.81$ (hexane/EtOAc, 1/2).

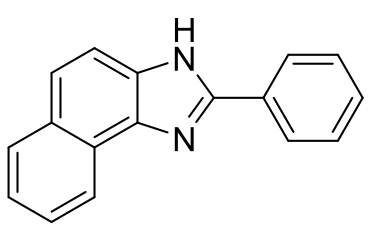

3t (2-phenyl-3H-naphtho[1,2- $d$ ]imidazole) ${ }^{\mathrm{S} 19}$ : yellow solid (110 mg, 90\%); ${ }^{\mathbf{1}} \mathbf{H}$ NMR (600 MHz, DMSO-d $\left.{ }_{6}\right) \delta 8.53(\mathrm{~d}, J=8.0 \mathrm{~Hz}, 1 \mathrm{H}), 8.26(\mathrm{~d}, J=7.6 \mathrm{~Hz}$, 2H), $8.00(\mathrm{~d}, J=7.8 \mathrm{~Hz}, 1 \mathrm{H}), 7.79-7.74(\mathrm{~m}, 1 \mathrm{H}), 7.71(\mathrm{~d}, J=8.5 \mathrm{~Hz}, 1 \mathrm{H})$, $7.63(\mathrm{t}, J=7.4 \mathrm{~Hz}, 1 \mathrm{H}), 7.58(\mathrm{dd}, J=8.0,7.8 \mathrm{~Hz}, 2 \mathrm{H}), 7.49(\mathrm{dd}, J=7.6,7.4 \mathrm{~Hz}$, 2H); ${ }^{13}$ C NMR (151 MHz, DMSO-d $) \delta$ 149.6, 139.0, 130.4, 130.0, 129.4, 129.1, 129.0, 128.8, 128.6, 126.2, 126.1, 124.3, 123.1, 121.5, 119.6; IR (neat): $v_{\max }=3441,1664,1463,1296,1153,1025,623 \mathrm{~cm}^{-1}$; $\mathrm{R}_{f} 0.77$ (hexane/EtOAc, 1/2)

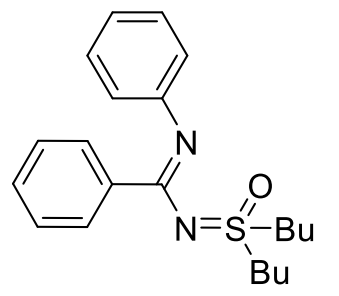

4a ( $N$-(dibutyl(oxo)- $\lambda^{6}$-sulfanylidene)- $N$-phenylbenzimidamide) : colorless liquid (41 mg, 38\%); ${ }^{1} \mathbf{H}$ NMR (600 MHz, $\left.\mathbf{C D C l}_{3}\right) \delta 7.33(\mathrm{~d}, J=7.3 \mathrm{~Hz}, 2 \mathrm{H}), 7.21(\mathrm{t}, J=$ $7.2 \mathrm{~Hz}, 1 \mathrm{H}), 7.17(\mathrm{dd}, J=7.3,7.2 \mathrm{~Hz}, 2 \mathrm{H}), 7.11(\mathrm{dd}, J=7.1,7.0 \mathrm{~Hz}, 2 \mathrm{H}), 6.87$ (t, $J=7.0 \mathrm{~Hz}, 1 \mathrm{H}), 6.64(\mathrm{~d}, J=7.1 \mathrm{~Hz}, 2 \mathrm{H}), 3.73-3.66(\mathrm{~m}, 2 \mathrm{H}), 3.50-3.43(\mathrm{~m}, 2 \mathrm{H})$, $1.93(\mathrm{tt}, J=7.6,7.4 \mathrm{~Hz}, 4 \mathrm{H}), 1.54(\mathrm{tq}, J=7.4,7.2 \mathrm{~Hz}, 4 \mathrm{H}), 1.00(\mathrm{t}, J=7.2 \mathrm{~Hz}, 6 \mathrm{H})$; ${ }^{13}$ C NMR (151 MHz, CDCl $) \delta$ 160.6, 152.8, 138.7, 129.7, 129.2, 128.8, 127.9, 122.4, 122.1, 51.0, 23.9, 22.0, 13.9; IR (neat): $v_{\max }=3060,2960,1587,1572,1463,1320,1272,1129,1027,698 \mathrm{~cm}^{-1}$; HRMS $\mathrm{m} / \mathrm{z}$ (EI) calc. for $\mathrm{C}_{21} \mathrm{H}_{28} \mathrm{~N}_{2} \mathrm{OS}\left[\mathrm{M}^{+}\right]$356.1922, found 356.1924; $\mathrm{R}_{f} 0.64$ (hexane/EtOAc, 3/1).

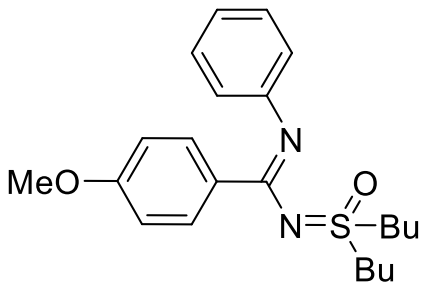

4d ( $N$-(dibutyl(oxo)- $\lambda^{6}$-sulfanylidene)-4-methoxy- $N$ '-phenylbenzimidamide) : colorless liquid (43 mg, 37\%); ${ }^{1} \mathbf{H}$ NMR (600 MHz, $\left.\mathbf{C D C l}_{3}\right) \delta 7.31(\mathrm{~d}$, $J=8.0 \mathrm{~Hz}, 2 \mathrm{H}), 7.13(\mathrm{dd}, J=7.4,7.1 \mathrm{~Hz}, 2 \mathrm{H}), 6.88(\mathrm{t}, J=7.1 \mathrm{~Hz}, 1 \mathrm{H})$, $6.67(\mathrm{~d}, J=8.0 \mathrm{~Hz}, 2 \mathrm{H}), 6.65(\mathrm{~d}, J=7.4 \mathrm{~Hz}, 2 \mathrm{H}), 3.73(\mathrm{~s}, 3 \mathrm{H}), 3.70-3.64$ $(\mathrm{m}, 2 \mathrm{H}), 3.48-3.40(\mathrm{~m}, 2 \mathrm{H}), 1.91(\mathrm{tt}, J=7.3,7.2 \mathrm{~Hz}, 4 \mathrm{H}), 1.53(\mathrm{qt}, J=7.6$, $7.3 \mathrm{~Hz}, 4 \mathrm{H}), 0.99$ (t, $J=7.6 \mathrm{~Hz}, 6 \mathrm{H}) ;{ }^{13} \mathbf{C}$ NMR (151 MHz, $\left.\mathbf{C D C l}_{3}\right) \delta 160.2,159.7,151.0,131.6,128.8$, 122.2, 121.8, 113.8, 113.1, 55.4, 50.9, 23.9, 21.9, 13.9; IR (neat): $v_{\max }=2960,1605,1511,1315,1251$, 1118, $695 \mathrm{~cm}^{-1}$; HRMS m/z (EI) calc. for $\mathrm{C}_{22} \mathrm{H}_{30} \mathrm{~N}_{2} \mathrm{O}_{2} \mathrm{~S}\left[\mathrm{M}^{+}\right]$386.2028, found 386.2030; $\mathrm{R}_{f} 0.45$ (hexane/EtOAc, 3/1). 


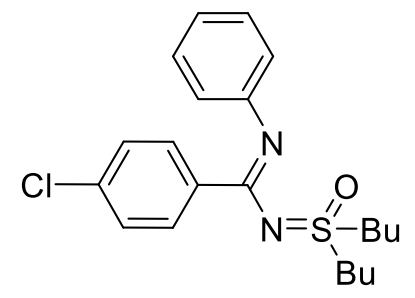

4e (4-chloro- $N$-(dibutyl(oxo)- $\lambda^{6}$-sulfanylidene)- $N$ '-phenylbenzimidamide) : colorless liquid (40 mg, 34\%); ${ }^{1} \mathbf{H}$ NMR (600 MHz, $\left.\mathbf{C D C l}_{3}\right) \delta 7.27$ (d, $J=7.3$ $\mathrm{Hz}, 2 \mathrm{H}), 7.15-7.11(\mathrm{~m}, 4 \mathrm{H}), 6.89(\mathrm{t}, J=7.4 \mathrm{~Hz}, 1 \mathrm{H}), 6.62(\mathrm{~d}, J=7.4 \mathrm{~Hz}$, $2 \mathrm{H}), 3.69-3.64(\mathrm{~m}, 2 \mathrm{H}), 3.48-3.43(\mathrm{~m}, 2 \mathrm{H}), 1.91(\mathrm{tt}, J=7.9,7.4 \mathrm{~Hz}, 4 \mathrm{H})$, $1.53(\mathrm{tq}, J=7.4,7.2 \mathrm{~Hz}, 4 \mathrm{H}), 0.99(\mathrm{~d}, J=7.2 \mathrm{~Hz}, 6 \mathrm{H}) ;{ }^{13} \mathbf{C}$ NMR (151 MHz, $\left.\mathbf{C D C l}_{3}\right) \delta 159.3,150.2,135.1,134.2,131.2,128.9,128.1,122.3,122.3,51.0,23.9,21.9,13.9$; IR (neat): $v_{\max }=2959,1737,1465,1373,1236,1032,635 \mathrm{~cm}^{-1} ; \mathrm{HRMS} \mathrm{m} / \mathrm{z}(\mathrm{EI})$ calc. for $\mathrm{C}_{21} \mathrm{H}_{27} \mathrm{ClN}_{2} \mathrm{OS}\left[\mathrm{M}^{+}\right]$ 390.1533, found 390.1530; $\mathrm{R}_{f} 0.68$ (hexane/EtOAc, 3/1).

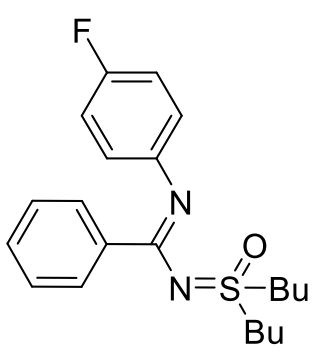

$4 \mathbf{m} \quad\left(N\right.$-(dibutyl(oxo)- $\lambda^{6}$-sulfanylidene)- $N$-(4-fluorophenyl)benzimidamide) $\quad$ : colorless liquid (45 mg, 40\%); ${ }^{1} \mathbf{H}$ NMR (600 MHz, $\left.\mathbf{C D C l}_{3}\right) \delta 7.29(\mathrm{~d}, J=7.4 \mathrm{~Hz}$, $2 \mathrm{H}), 7.23(\mathrm{t}, J=7.4 \mathrm{~Hz}, 1 \mathrm{H}), 7.18(\mathrm{dd}, J=7.4,7.4 \mathrm{~Hz}, 2 \mathrm{H}), 6.79(\mathrm{dd}, J=8.6 \mathrm{~Hz}$, $\left.{ }^{3} J_{H-F}=8.6 \mathrm{~Hz}, 2 \mathrm{H}\right), 6.55\left(\mathrm{dd}, J=8.6 \mathrm{~Hz},{ }^{3} J_{H-F}=5.0 \mathrm{~Hz}, 2 \mathrm{H}\right), 3.67-3.61(\mathrm{~m}, 2 \mathrm{H})$, $3.51-3.45(\mathrm{~m}, 2 \mathrm{H}), 1.92(\mathrm{tt}, J=7.9,7.7 \mathrm{~Hz}, 4 \mathrm{H}), 1.54(\mathrm{tq}, J=7.7,7.4 \mathrm{~Hz}, 4 \mathrm{H})$, $1.00(\mathrm{t}, J=7.4 \mathrm{~Hz}, 6 \mathrm{H}) ;{ }^{13} \mathbf{C}$ NMR (151 MHz, $\left.\mathbf{C D C l}_{3}\right) \delta 161.3,158.6\left(\mathrm{~d},{ }^{1} J_{C-F}=\right.$ $240.3 \mathrm{~Hz}), 146.7,135.9,129.5,129.1,127.9,123.4\left(\mathrm{~d},{ }^{3} J_{C-F}=7.8 \mathrm{~Hz}\right), 115.3\left(\mathrm{~d},{ }^{2} J_{C-F}=22.2 \mathrm{~Hz}\right), 51.0$, 24.1, 22.0, 13.9; IR (neat): $v_{\max }=2961,1737,1589,1499,1322,1240,1129,1030,701 \mathrm{~cm}^{-1} ; \mathrm{HRMS} \mathrm{m} / \mathrm{z}$ (EI) calc. for $\mathrm{C}_{21} \mathrm{H}_{27} \mathrm{FN}_{2} \mathrm{OS}\left[\mathrm{M}^{+}\right]$374.1828, found 374.1827; $\mathrm{R}_{f} 0.59$ (hexane/EtOAc, 3/1).

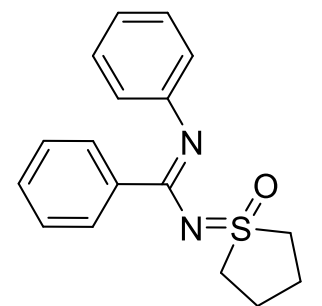

5a $\left(N\right.$-(1-oxidotetrahydro- $1 \lambda^{6}$-thiophen-1-ylidene)- $N$ '-phenylbenzimidamide) : white solid (43 mg, 48\%), m.p. 89-94 ${ }^{\circ} \mathrm{C}$; ${ }^{1} \mathbf{H}$ NMR (600 MHz, $\left.\mathbf{C D C l}_{3}\right) \delta 7.34(\mathrm{~d}, J=7.3$ $\mathrm{Hz}, 2 \mathrm{H}), 7.23(\mathrm{t}, J=7.0 \mathrm{~Hz}, 1 \mathrm{H}), 7.18(\mathrm{dd}, J=7.3,7.0 \mathrm{~Hz}, 2 \mathrm{H}), 7.11(\mathrm{dd}, J=7.4$, $7.2 \mathrm{~Hz}, 2 \mathrm{H}), 6.89(\mathrm{t}, J=7.2 \mathrm{~Hz}, 1 \mathrm{H}), 6.69$ (d, $J=7.4 \mathrm{~Hz}, 2 \mathrm{H}), 3.86(\mathrm{ddd}, J=13.0$, 6.4, $6.4 \mathrm{~Hz}, 2 \mathrm{H}), 3.31(\mathrm{ddd}, J=13.0,6.3,6.3 \mathrm{~Hz}, 2 \mathrm{H}), 2.39-2.26(\mathrm{~m}, 4 \mathrm{H}) ;{ }^{13} \mathrm{C}$ NMR (151 MHz, CDCl $) \delta 161.1,150.3,135.4,129.6,129.2,128.7,127.9,122.5,122.2,52.6,24.1$; IR (neat): $v_{\max }=3056,2947,1572,1320,1268,1204,1120,698 \mathrm{~cm}^{-1} ; \mathrm{HRMS} \mathrm{m} / \mathrm{z}$ (EI) calc. for $\mathrm{C}_{17} \mathrm{H}_{18} \mathrm{~N}_{2} \mathrm{OS}$ $\left[\mathrm{M}^{+}\right]$298.1140, found 298.1141; $\mathrm{R}_{f} 0.48$ (hexane/EtOAc, 1/2).

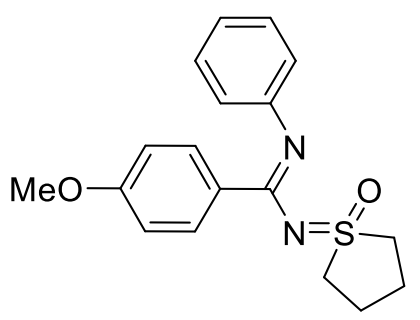

5d (4-methoxy- $N$-(1-oxidotetrahydro-1 $\lambda^{6}$-thiophen-1-ylidene)- $N$-phenylbenzimidamide) : white solid (49 $\mathrm{mg}, 50 \%) ;{ }^{1} \mathbf{H}$ NMR (600 $\left.\mathbf{M H z}, \mathbf{C D C l}_{3}\right) \delta$ $7.32(\mathrm{~d}, J=8.6 \mathrm{~Hz}, 2 \mathrm{H}), 7.14(\mathrm{dd}, J=7.9,7.1 \mathrm{~Hz}, 2 \mathrm{H}), 6.90(\mathrm{t}, J=7.1 \mathrm{~Hz}$, $1 \mathrm{H}), 6.71(\mathrm{~d}, J=8.6 \mathrm{~Hz}, 2 \mathrm{H}), 6.69(\mathrm{~d}, J=7.9 \mathrm{~Hz}, 2 \mathrm{H}), 3.85$ (ddd, $J=12.5$, 
5.9, $5.9 \mathrm{~Hz}, 2 \mathrm{H}), 3.75(\mathrm{~s}, 3 \mathrm{H}), 3.28(\mathrm{ddd}, J=12.5,6.0,6.0 \mathrm{~Hz}, 2 \mathrm{H}), 2.39-2.26(\mathrm{~m}, 4 \mathrm{H}) ;{ }^{13} \mathrm{C}$ NMR (151 MHz, $\left.\mathbf{C D C l}_{3}\right) \delta 160.4,160.3,150.6,131.6,128.8,122.4,122.1,113.2,110.2,55.4,52.5,24.1$; IR (neat): $v_{\max }=2977,2254,1588,1382,903,723 \mathrm{~cm}^{-1}$; HRMS m/z (EI) calc. for $\mathrm{C}_{18} \mathrm{H}_{20} \mathrm{~N}_{2} \mathrm{O}_{2} \mathrm{~S}\left[\mathrm{M}^{+}\right] 328.1245$, found 328.1248; $\mathrm{R}_{f} 0.38$ (hexane/EtOAc, 1/2).

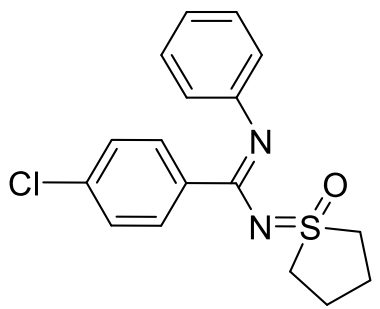

5e (4-chloro- $N$-(1-oxidotetrahydro-1 $\lambda^{6}$-thiophen-1-ylidene)- $N$ '-phenylbenzimidamide) : white solid (41 mg, 41\%), m.p. 100-106 ${ }^{\circ} \mathrm{C} ;{ }^{1} \mathbf{H}$ NMR (600 MHz, $\left.\mathbf{C D C l}_{3}\right) \delta 7.28(\mathrm{~d}, J=8.3 \mathrm{~Hz}, 2 \mathrm{H}), 7.15(\mathrm{~d}, J=8.3 \mathrm{~Hz}, 2 \mathrm{H}), 7.13(\mathrm{dd}, J=7.5$, $7.1 \mathrm{~Hz}, 2 \mathrm{H}), 6.91(\mathrm{t}, J=7.1 \mathrm{~Hz}, 1 \mathrm{H}), 6.67(\mathrm{~d}, J=7.5 \mathrm{~Hz}, 2 \mathrm{H}), 3.84(\mathrm{ddd}, J=$ $12.5,6.3,6.3 \mathrm{~Hz}, 2 \mathrm{H}), 3.30(\mathrm{ddd}, J=12.3,6.0,6.0 \mathrm{~Hz}, 2 \mathrm{H}), 2.39-2.25(\mathrm{~m}$, 4H); ${ }^{13} \mathbf{C}$ NMR (151 MHz, $\left.\mathbf{C D C l}_{3}\right) \delta 159.8,150.0,135.2,133.7,131.2,128.9,128.2,122.5,122.3,52.6$, 24.1; IR (neat): $v_{\max }=2949,2253,1585,1205,904,727 \mathrm{~cm}^{-1}$; HRMS m/z (EI) calc. for $\mathrm{C}_{17} \mathrm{H}_{17} \mathrm{ClN}_{2} \mathrm{OS}$ $\left[\mathrm{M}^{+}\right]$332.0750, found 332.0750; $\mathrm{R}_{f} 0.51$ (hexane/EtOAc, 1/2).

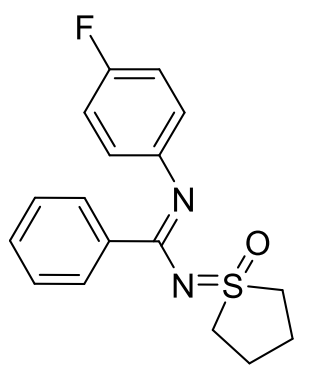

$5 \mathbf{m}$ ( $N$-(4-fluorophenyl)- $N$-(1-oxidotetrahydro-1 $\lambda^{6}$-thiophen-1-ylidene)benzimidamide) : pale yellow solid (41 mg, 43\%), m.p. 113-119 ${ }^{\circ} \mathrm{C} ;{ }^{1} \mathbf{H}$ NMR (600 MHz, $\left.\mathbf{C D C l}_{3}\right) \delta 7.30(\mathrm{~d}, J=7.1 \mathrm{~Hz}, 2 \mathrm{H}), 7.24(\mathrm{t}, J=7.4 \mathrm{~Hz}, 1 \mathrm{H}), 7.20(\mathrm{dd}, J=7.4,7.1 \mathrm{~Hz}$, $2 \mathrm{H}), 6.80\left(\mathrm{dd}, J=8.7 \mathrm{~Hz},{ }^{3} J_{H-F}=8.6 \mathrm{~Hz}, 2 \mathrm{H}\right), 6.61\left(\mathrm{dd}, J=8.7 \mathrm{~Hz},{ }^{4} J_{H-F}=5.0 \mathrm{~Hz}\right.$, 2H), 3.84 (ddd, $J=13.1,6.9,6.9 \mathrm{~Hz}, 2 \mathrm{H}), 3.33$ (ddd, $J=13.1,6.7,6.7 \mathrm{~Hz}, 2 \mathrm{H}$ ), $2.38-2.26(\mathrm{~m}, 4 \mathrm{H}) ;{ }^{13} \mathbf{C}$ NMR (151 MHz, $\left.\mathbf{C D C l}_{3}\right) \delta 161.8,158.8\left(\mathrm{~d},{ }^{l} J_{C-F}=241.6\right.$ $\mathrm{Hz}), 146.3,135.3,129.5,129.3,128.0,123.6\left(\mathrm{~d},{ }^{3} J_{C-F}=7.5 \mathrm{~Hz}\right), 115.3\left(\mathrm{~d},{ }^{2} J_{C-F}=22.6 \mathrm{~Hz}\right), 52.8,24.1$; IR (neat): $v_{\max }=2948,2250,1571,1498,1304,1204,1120,907,839,730 \mathrm{~cm}^{-1}$; HRMS m/z (EI) calc. for $\mathrm{C}_{17} \mathrm{H}_{17} \mathrm{FN}_{2} \mathrm{OS}\left[\mathrm{M}^{+}\right]$316.1046, found 316.1048; $\mathrm{R}_{f} 0.44$ (hexane/EtOAc, 1/2).

\section{References}

(S1) Sun, J.; Wu, W.; Zhao, J. Chem. Eur. J. 2012, 18, 8100.

(S2) Frisch, M. J.; Trucks, G. W.; Schlegel, H. B.; Scuseria, G. E.; Robb, M. A.; Cheeseman, J. R.; Scalmani, G.; Barone, V.; Mennucci, B.; Petersson, G. A.; Nakatsuji, H.; Caricato, M.; Li, X.; Hratchian, H. P.; Izmaylov, A. F.; Bloino, J.; Zheng, G.; Sonnenberg, J. L.; Hada, M.; Ehara, M.; Toyota, K.; Fukuda, R.; Hasegawa, J.; Ishida, M.; Nakajima, T.; Honda, Y.; Kitao, O.; Nakai, H.; Vreven, T.; Montgomery Jr., J. A.; Peralta, J. E.; Ogliaro, F.; Bearpark, 
M.; Heyd, J. J.; Brothers, E.; Kudin, K. N.; Staroverov, V. N.; Kobayashi, R.; Normand, J.; Raghavachari, K.; Rendell, A.; Burant, J. C.; Iyengar, S. S.; Tomasi, J.; Cossi, M.; Rega, N.; Millam, J. M.; Klene, M.; Knox, J. E.; Cross, J. B.; Bakken, V.; Adamo, C.; Jaramillo, J.;

Gomperts, R.; Stratmann, R. E.; Yazyev, O.; Austin, A. J.; Cammi, R.; Pomelli, C.; Ochterski, J. W.; Martin, R. L.; Morokuma, K.; Zakrzewski, V. G.; Voth, G. A.; Salvador, P.; Dannenberg, J. J.; Dapprich, S.; Daniels, A. D.; Farkas, O.; Foresman, J. B.; Ortiz, J. V.; Cioslowski, J.; Fox, D. J. Gaussian 09, Revision D01; Gaussian, Inc., Wallingford, CT, 2009. (S3) Zhao, Y.; Truhlar, D. G. Theor. Chem. Acc. 2008, 120, 215.

(S4) Tomasi, J.; Mennucci, B.; Cammi, R. Chem. Rev. 2005, 105, 2999.

(S5) Slagbrand, T.; Kervefors, G.; Tinnis, F.; Adolfsson, H. Adv. Synth. Catal. 2017, 359, 1990.

(S6) The imines were prepared by stirring an equimolar amount of aldehyde and amine in methanol $(0.2 \mathrm{M})$ at room temperature for $12-24 \mathrm{~h}$. The volatiles were removed under vacuum, and thus obtained imines were used without further purification.

(S7) Soni, V. K.; Hwang, H. S.; Moon, Y. K.; Park, S.-W.; You, Y.; Cho, E. J. J. Am. Chem. Soc. 2019, 141, 10538.

(S8) Alcaide, B.; Mardomingo, C. L.; Plumet, J.; Cativiela, C.; Mayoral, J. A. Can. J. Chem. 1987, 65, 2050.

(S9) Wang, Y.-F.; Zhang, F.-L.; Chiba, S. Org. Lett. 2013, 15, 2842.

(S10) Wang, Y.-F.; Zhu, X.; Chiba, S. J. Am. Chem. Soc. 2012, 134, 3679.

(S11) Yoshida, H.; Sogame, S.; Takishita, Y.; Ogata, T. Bull. Chem. Soc. Jpn. 1983, 56, 2438.

(S12) Park, S.; Jung, J.; Cho, E. J. Eur. J. Org. Chem. 2014, 2014, 4148.

(S13) Li, G.; He, R.; Liu, Q.; Wang, Z.; Liu, Y.; Wang, Q. J. Org. Chem. 2019, 84, 8646.

(S14) Li, Z.; Song, H.; Guo, R.; Zuo, M.; Hou, C.; Sun, S.; He, X.; Sun, Z.; Chu, W. Green Chem. 2019, 21, 3602.

(S15) Alunni Bistocchi, G.; De Meo, G.; Pedini, M.; Ricci, A.; Pitzurra, M.; Cavallo, R.; Sposini, T.; Jacquignon, P. Farmaco, Ed. Sci. 1982, 37, 597.

(S16) Qu, Y.; Pan, L.; Wu, Z.; Zhou, X. Tetrahedron 2013, 69, 1717.

(S17) Menteşe, E.; Kahveci, B.; Menteşe, M. J. Chem. Res. 2018, 42, 329.

(S18) Diao, X.; Wang, Y.; Jiang, Y.; Ma, D. J. Org. Chem. 2009, 74, 7974.

(S19) Gaber, A-A.; Taib, L. J. Chem. Sci. 2016, 128, 745. 

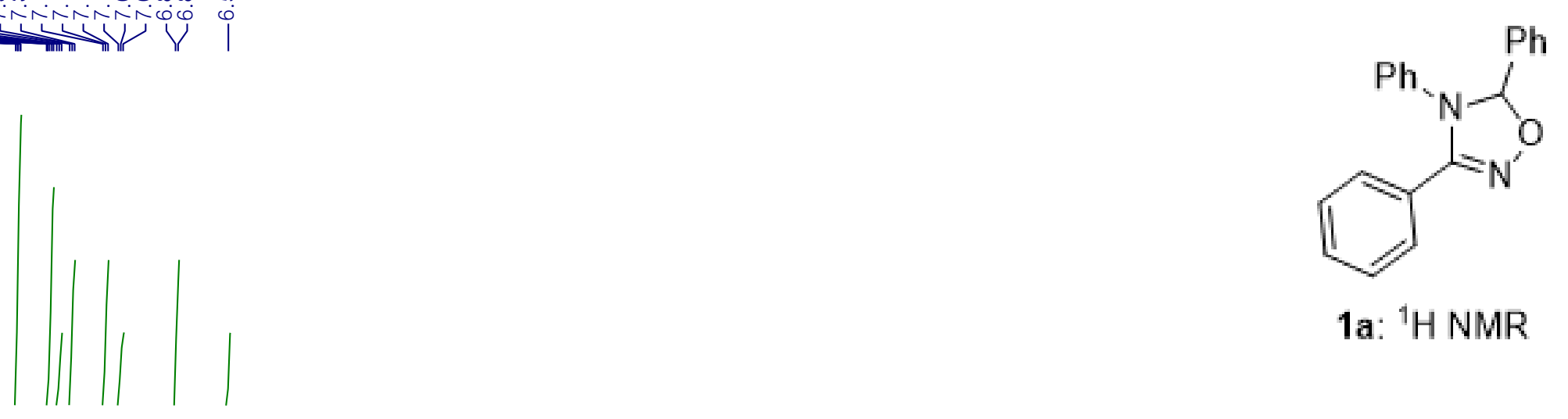

1a: ${ }^{1}$ H NMR

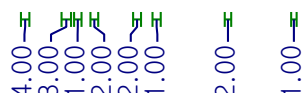
7.2
im 


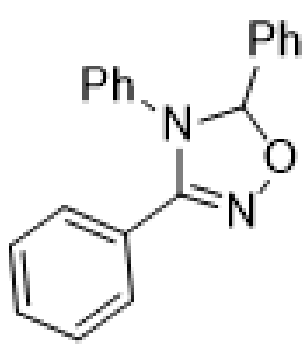

1a: ${ }^{13} \mathrm{C}$ NMR

1a. ${ }^{13}$ C NMR 


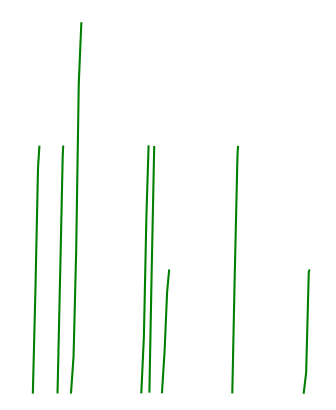

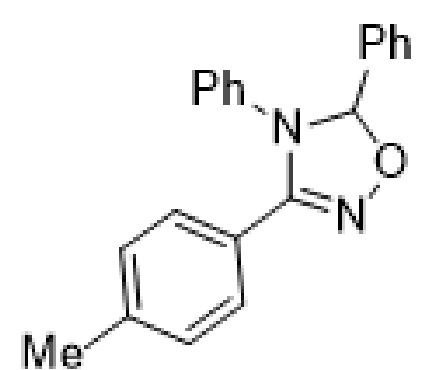

1b: ${ }^{1} \mathrm{H}$ NMR

the

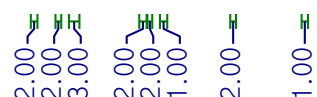

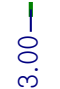

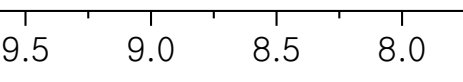

\begin{tabular}{ll|l}
7.5 & 1
\end{tabular}

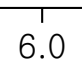




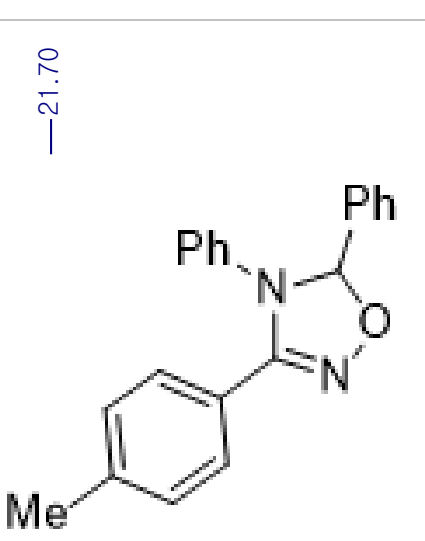

1b: ${ }^{13} \mathrm{C}$ NMR

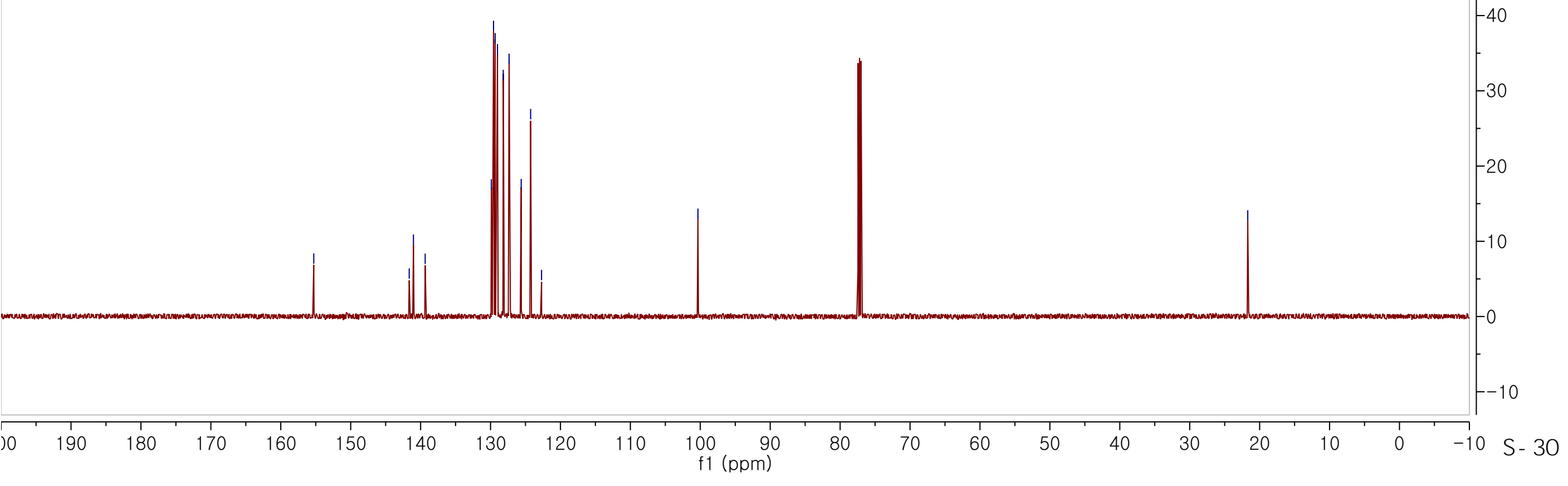




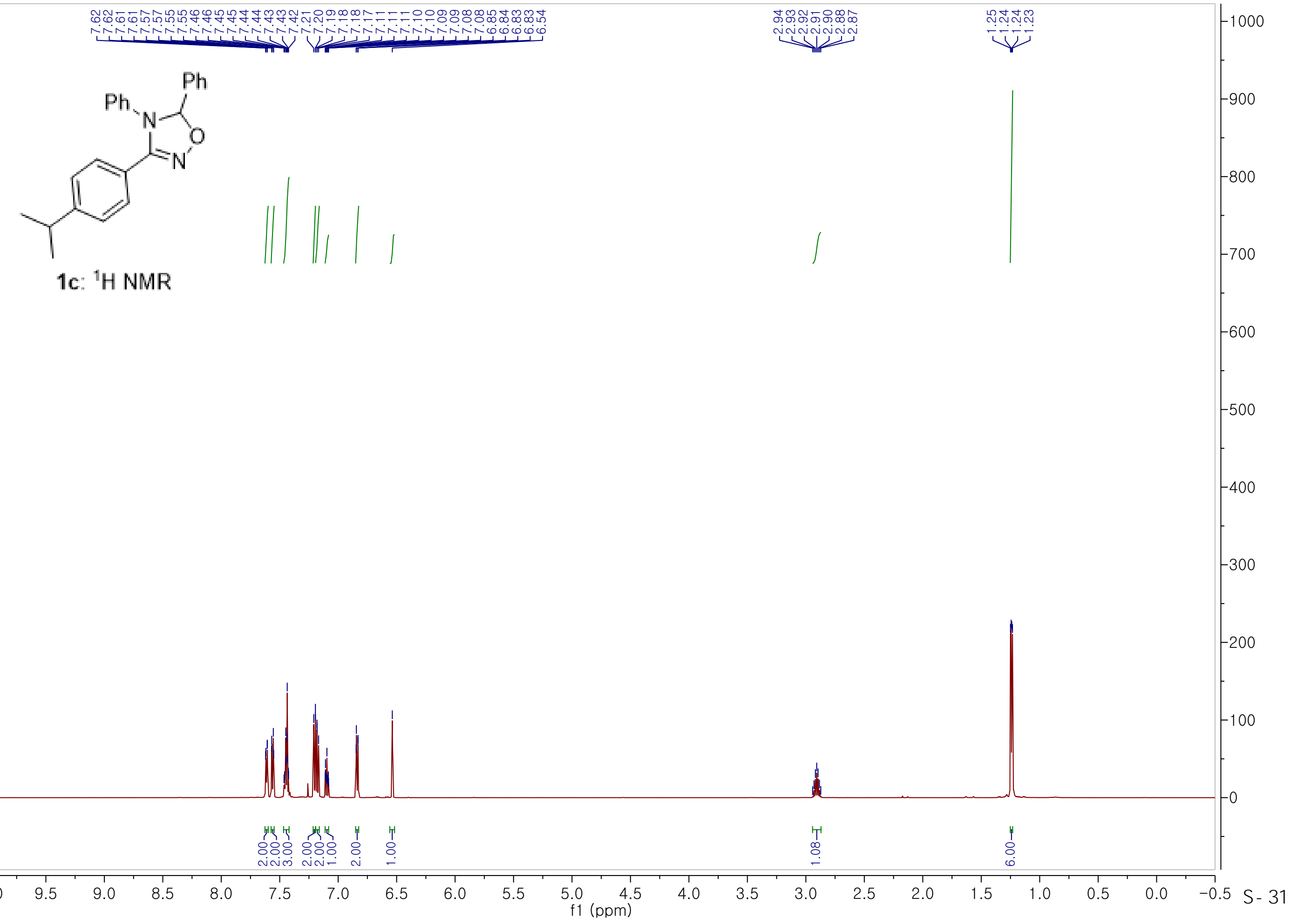




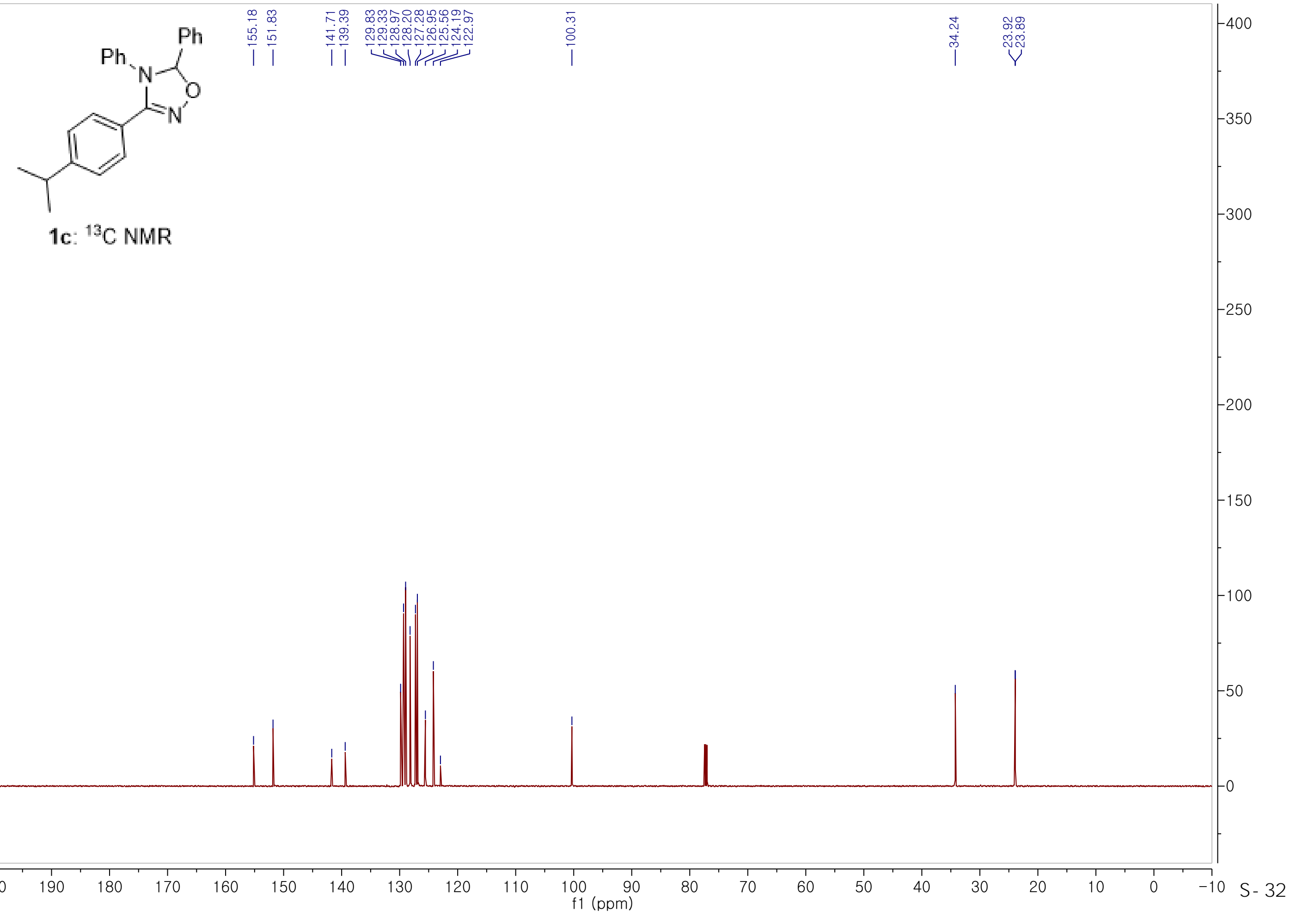




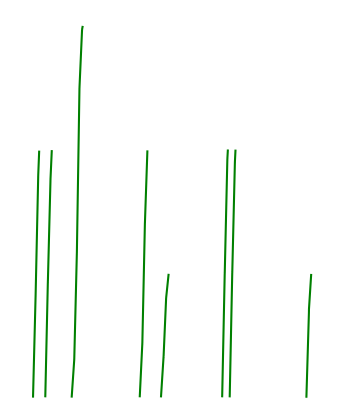

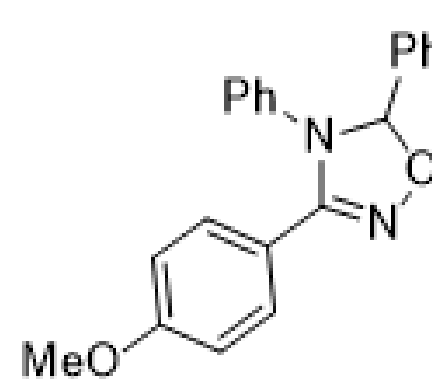

1d: ${ }^{1} \mathrm{H}$ NMR

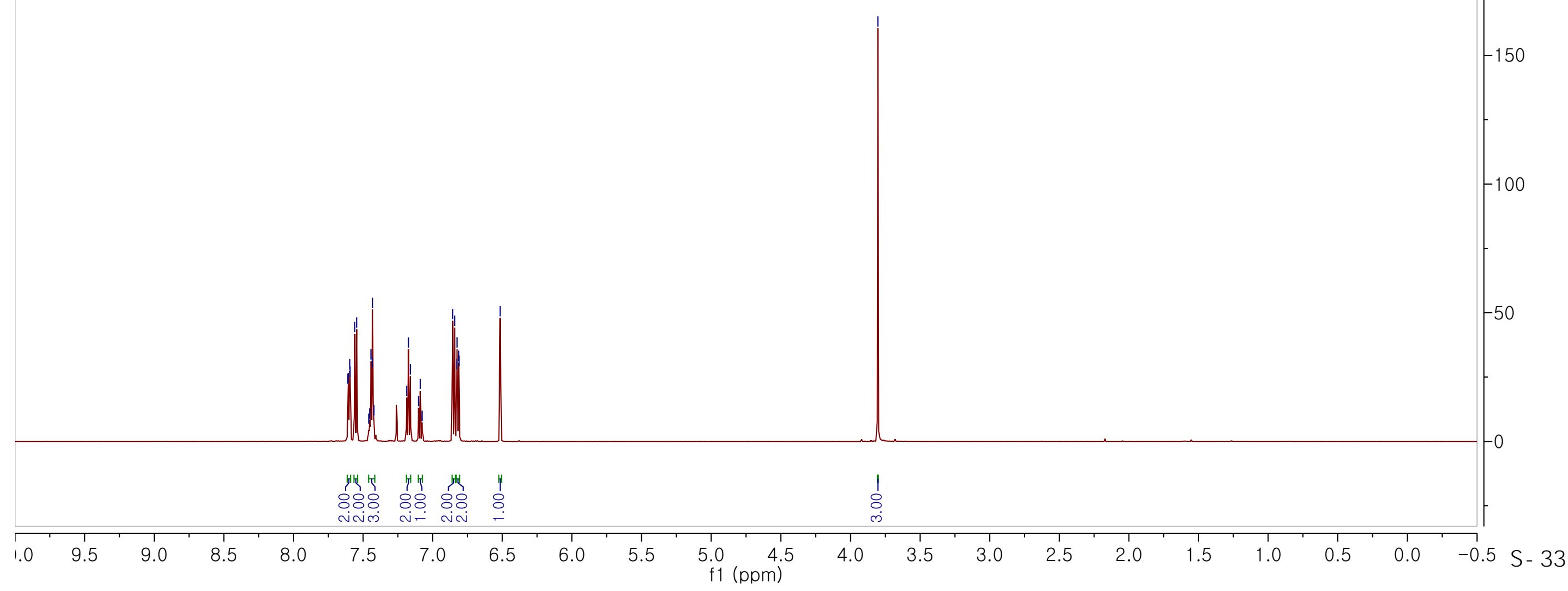




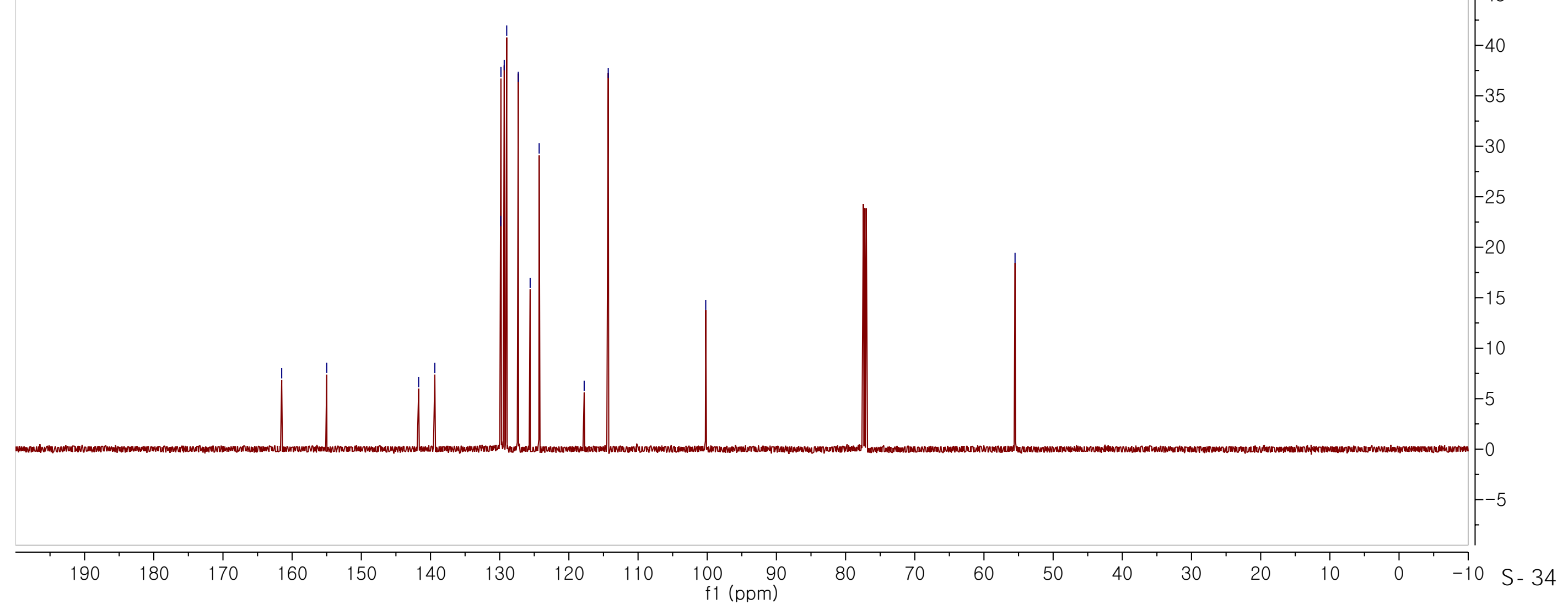




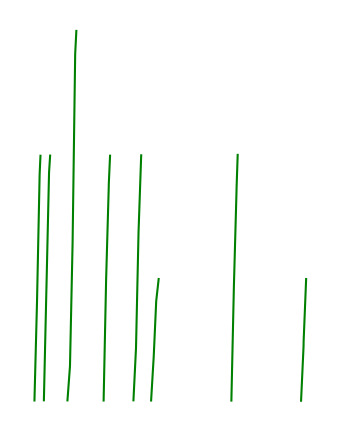

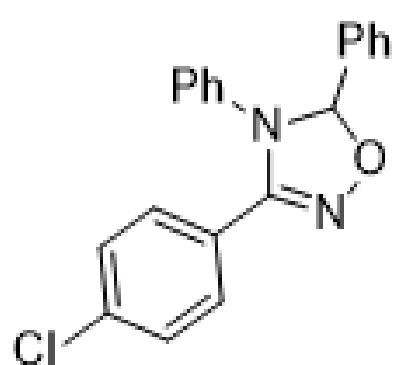

1e: ${ }^{1}$ H NMR

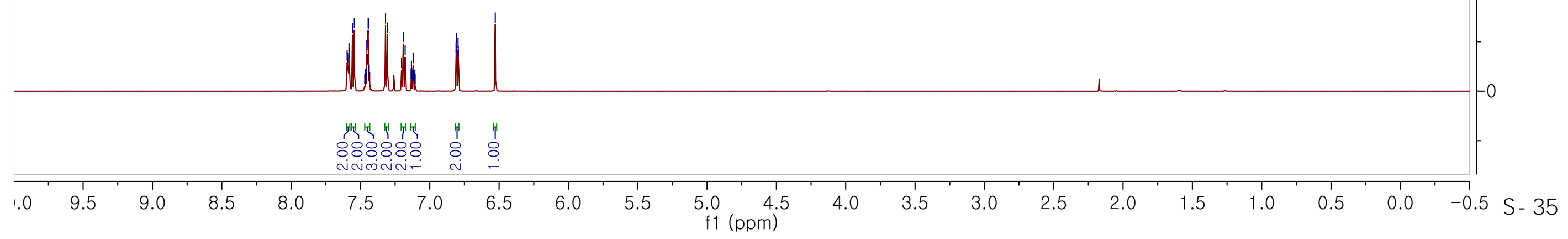




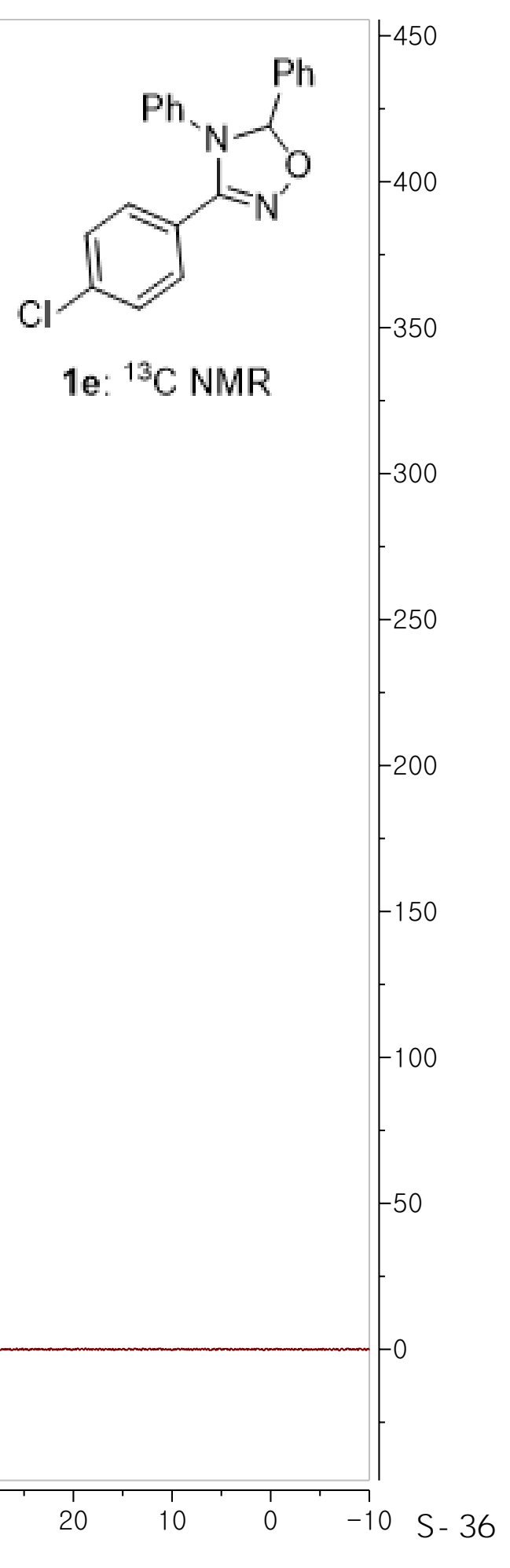




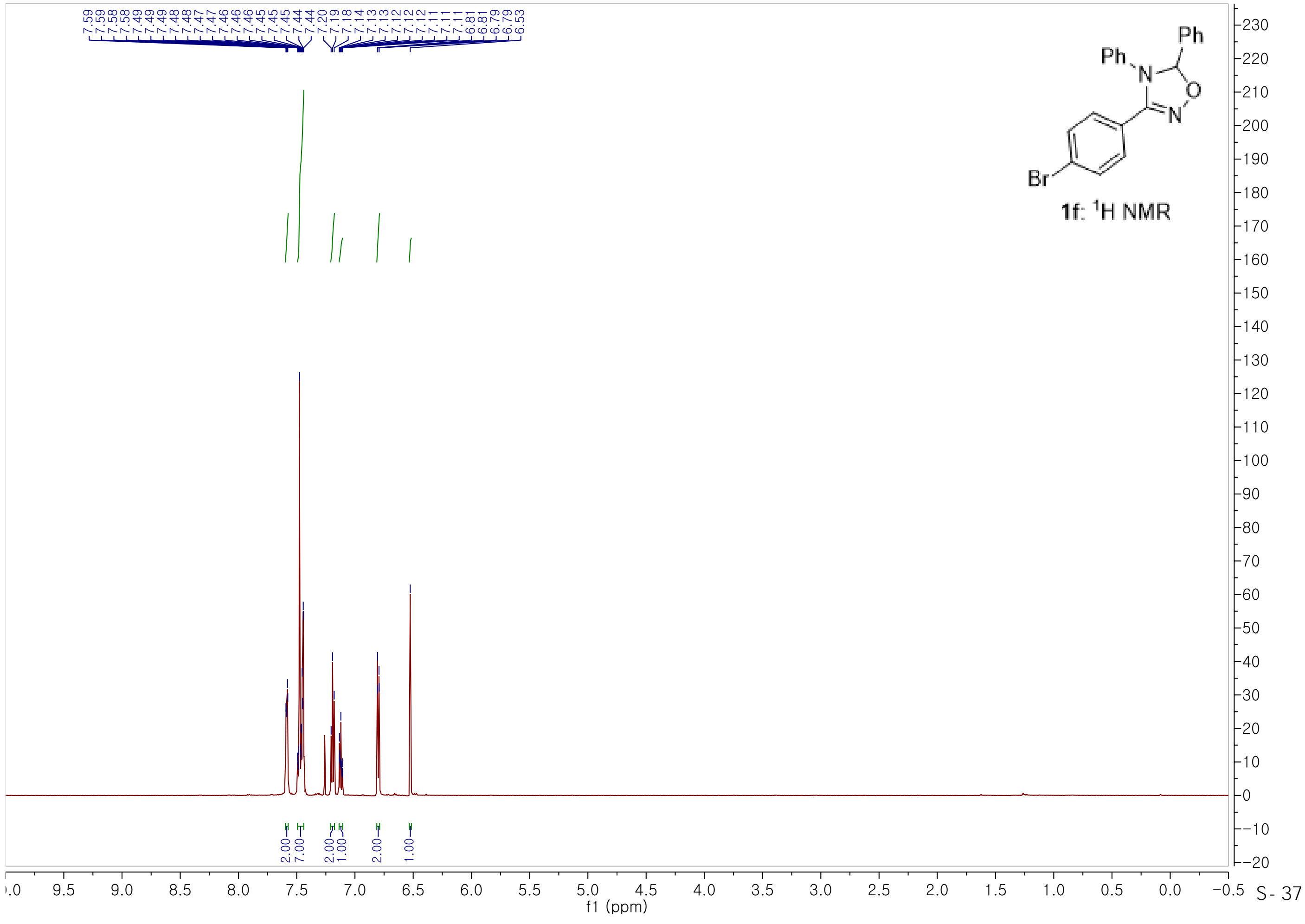




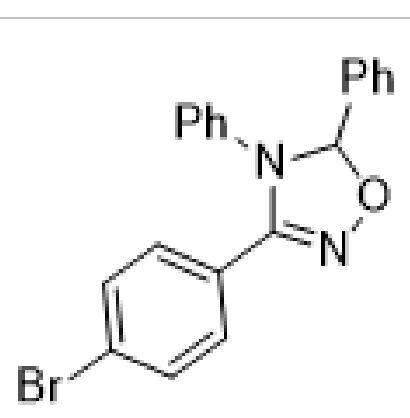

1f: ${ }^{13} \mathrm{C}$ NMR

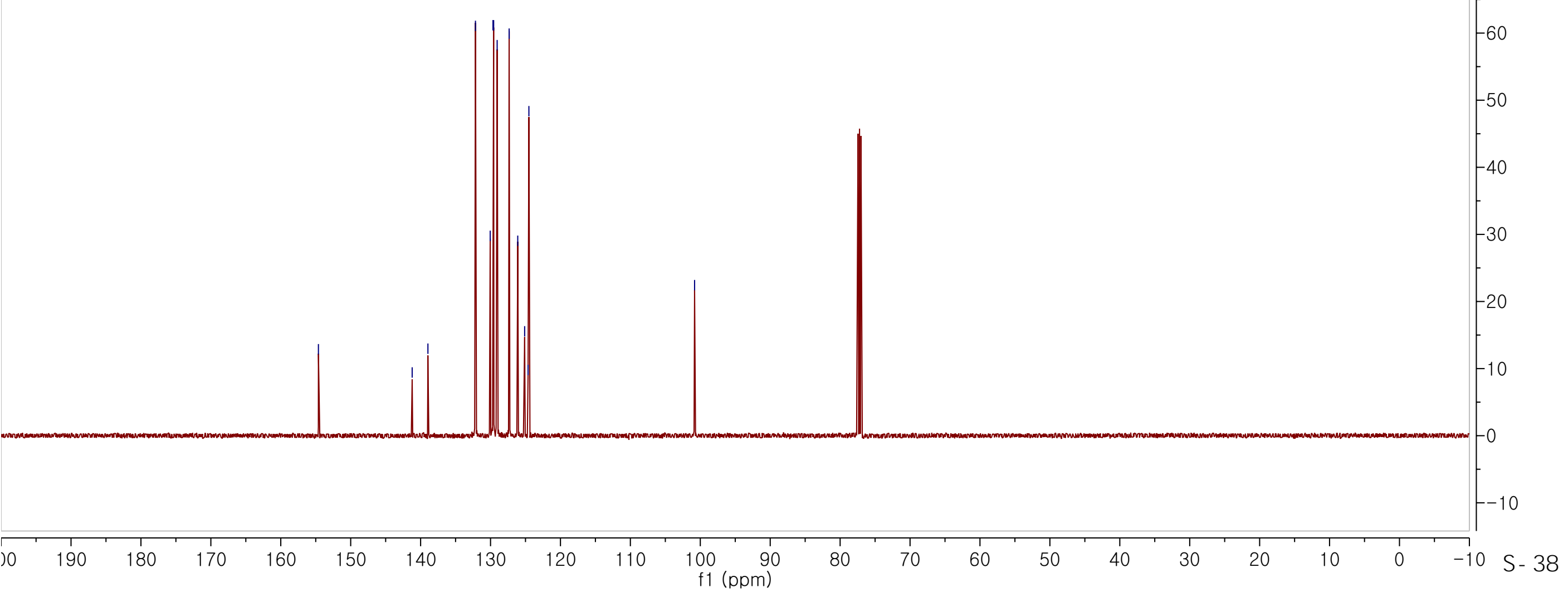



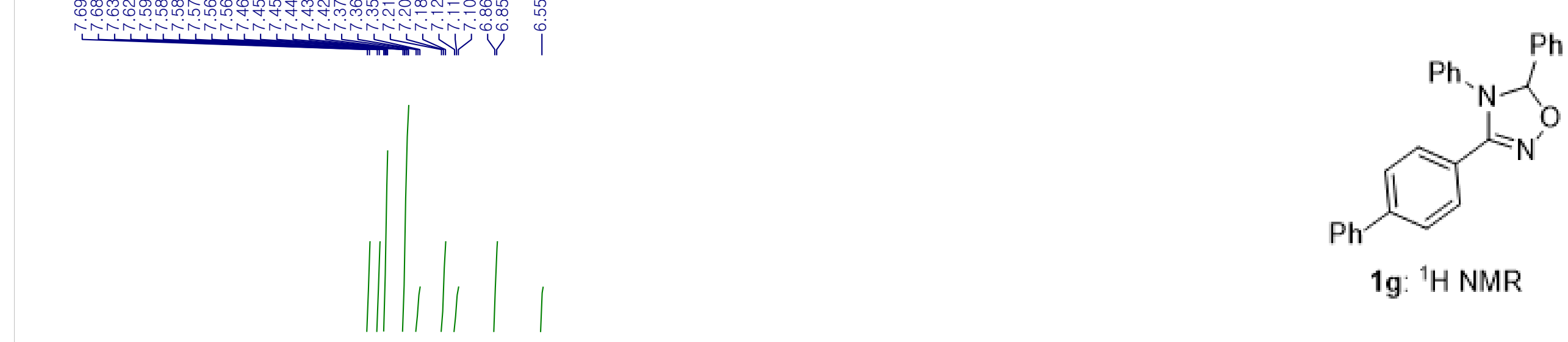

g: ${ }^{1} \mathrm{H}$ NMR 
<smiles>c1ccc(-c2ccc(C3=NOC(c4ccccc4)N3c3ccccc3)cc2)cc1</smiles>

1g: ${ }^{13} \mathrm{C}$ NMR

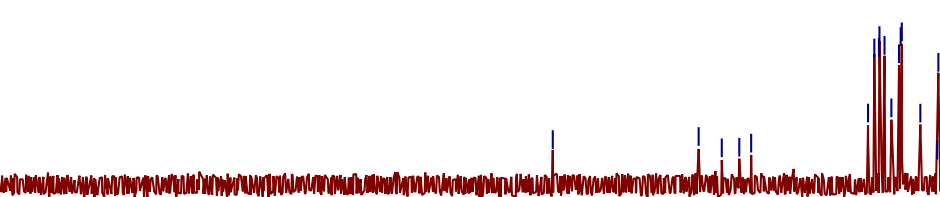



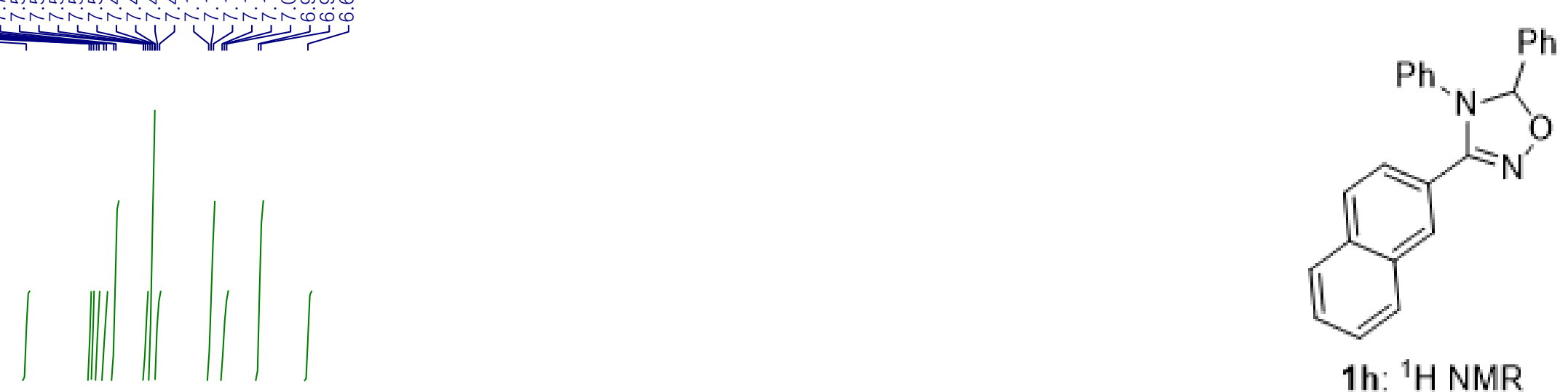

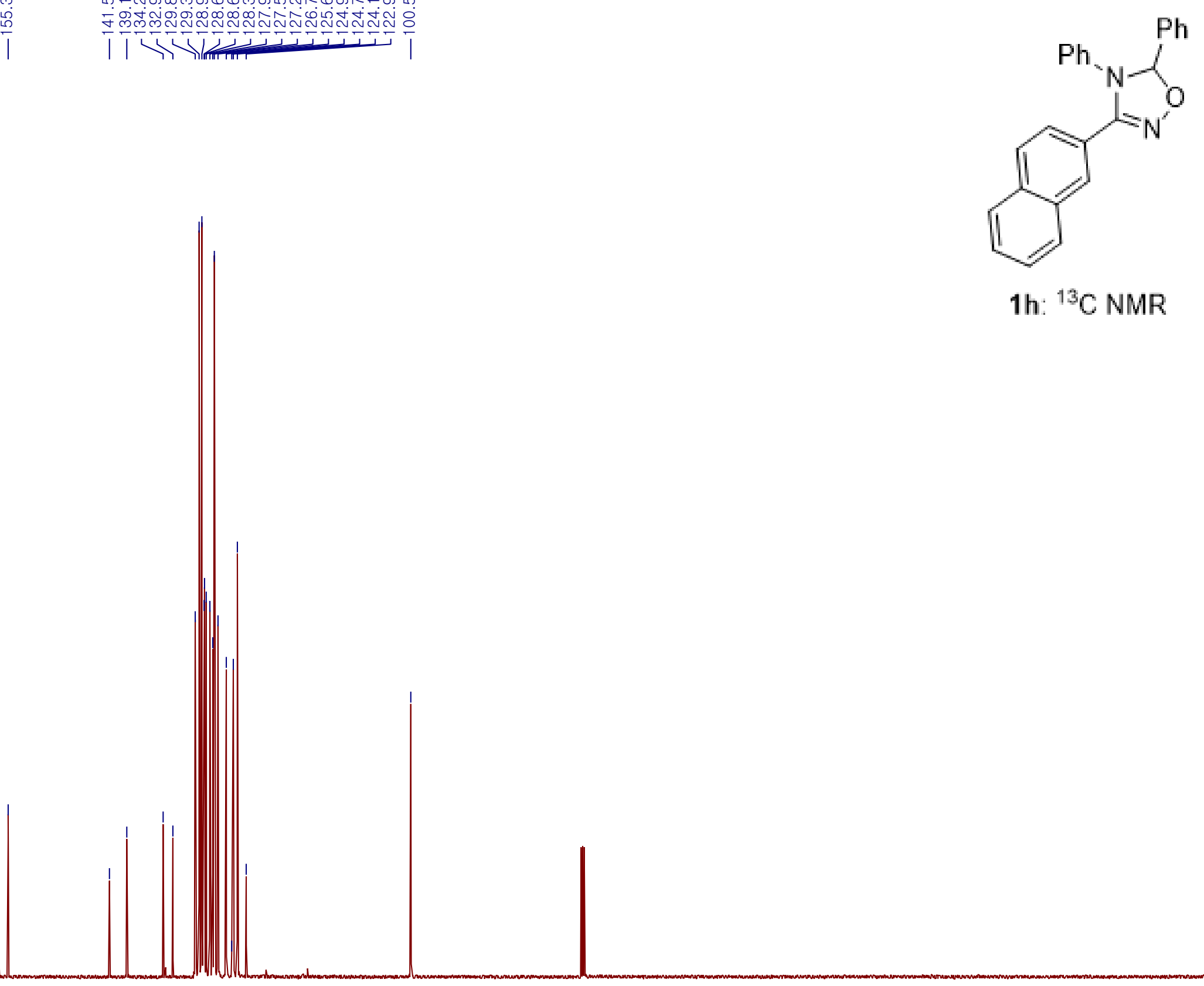

1h: ${ }^{13} \mathrm{C}$ NMR 

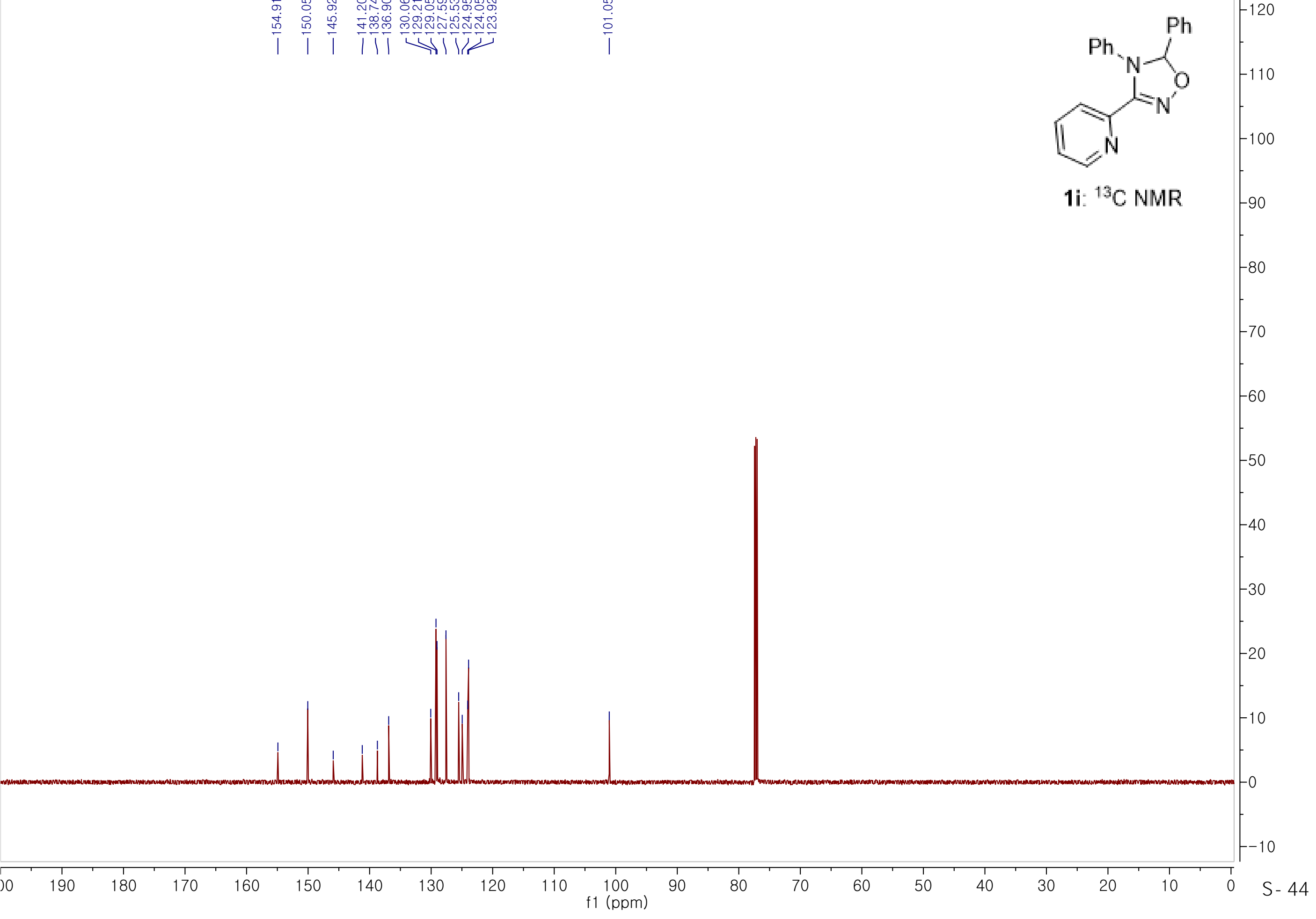


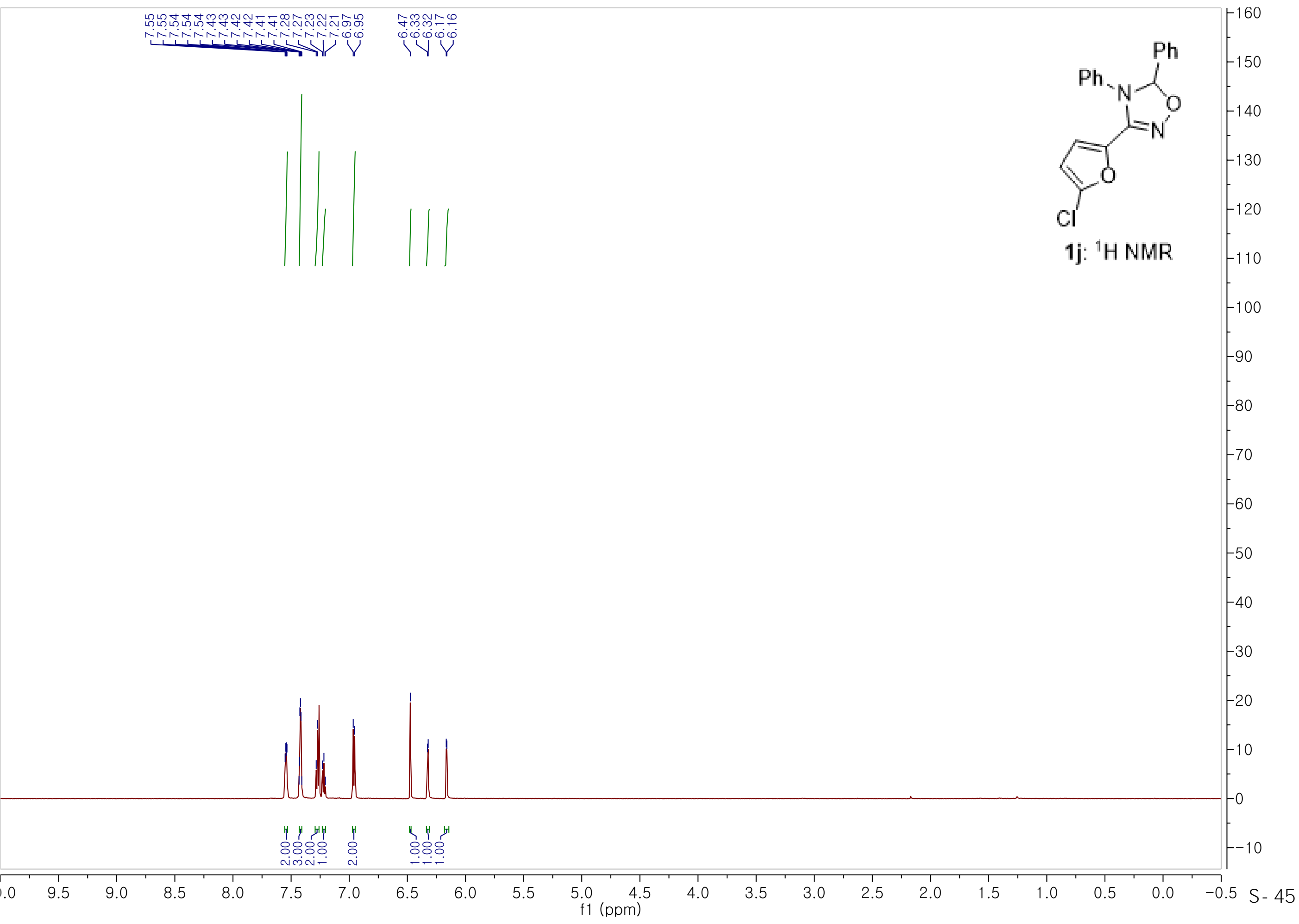




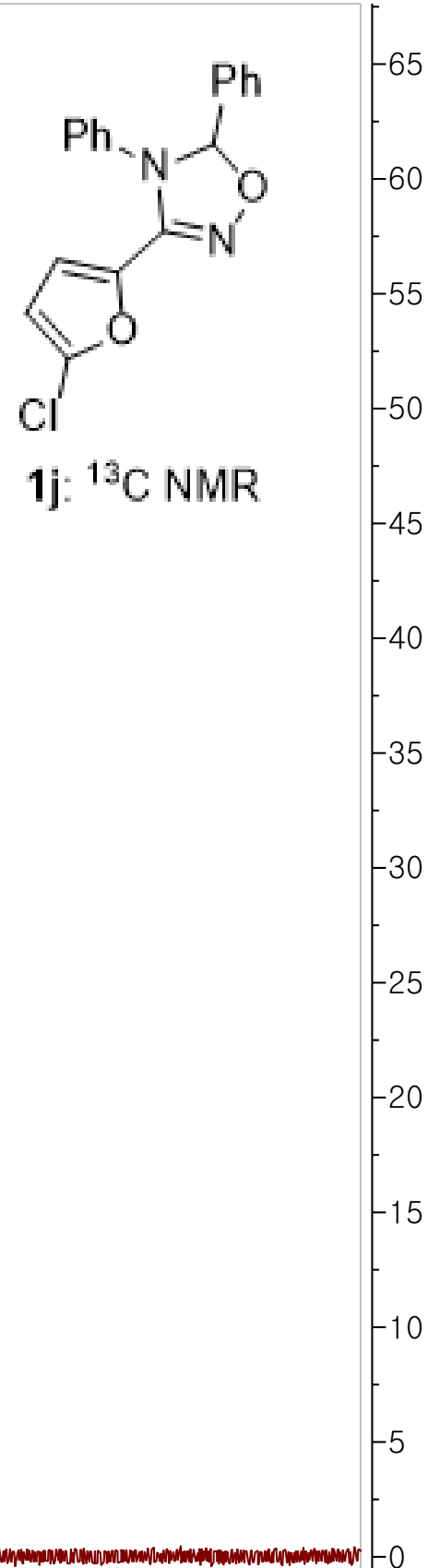




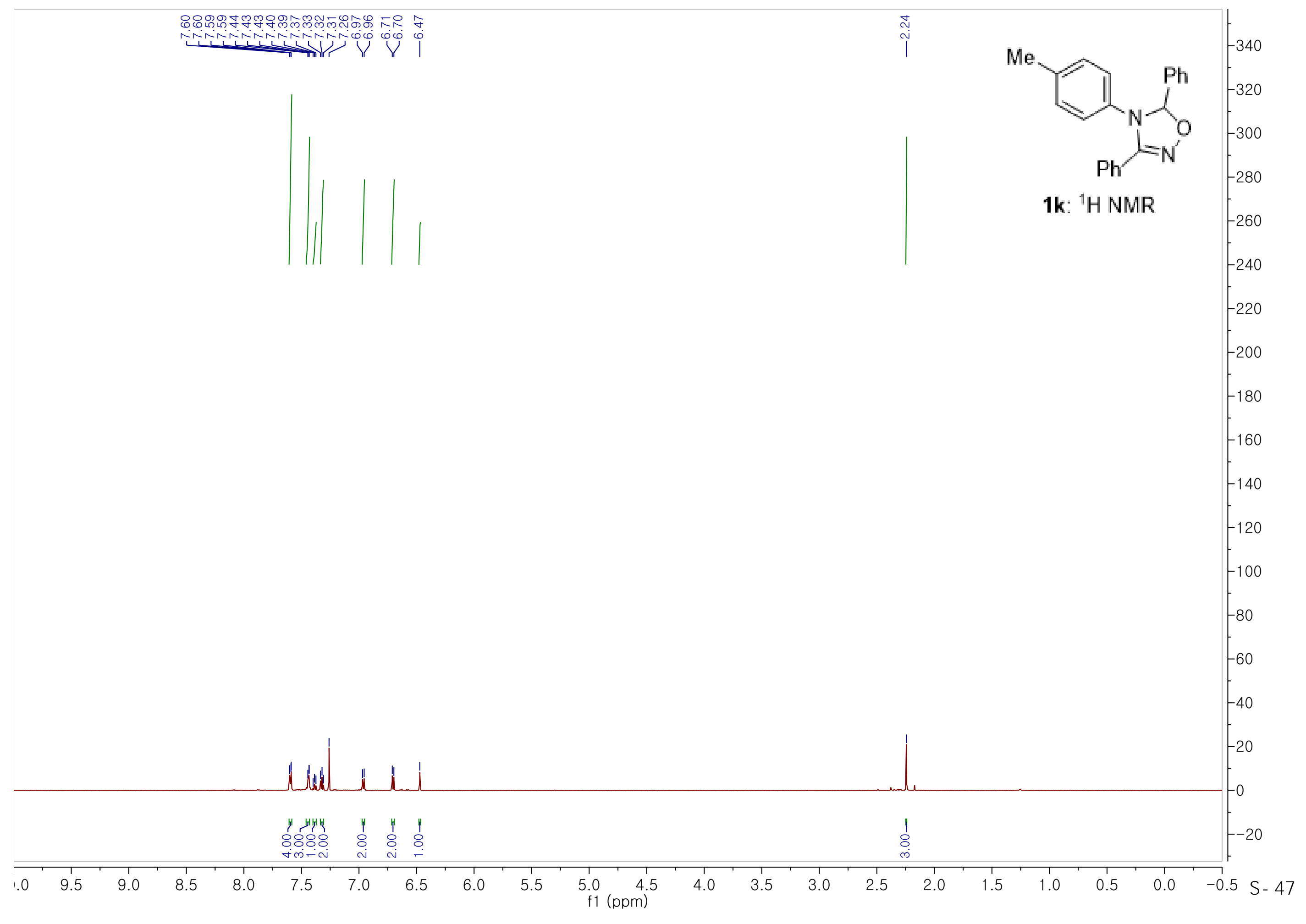


Me<smiles>Cc1ccc(N2C(c3ccccc3)=NOC2c2ccccc2)cc1</smiles>

1k: ${ }^{1} \mathrm{H}$ NMR 


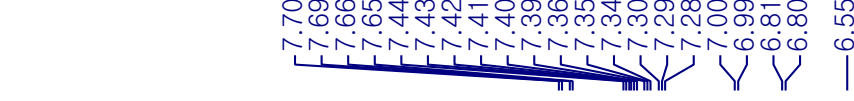

\section{$\mathrm{Bu}$}<smiles>Ic1ccc(N2C(c3ccccc3)=NOC2c2ccccc2)cc1</smiles>

1I: ${ }^{1} \mathrm{H}$ NMR
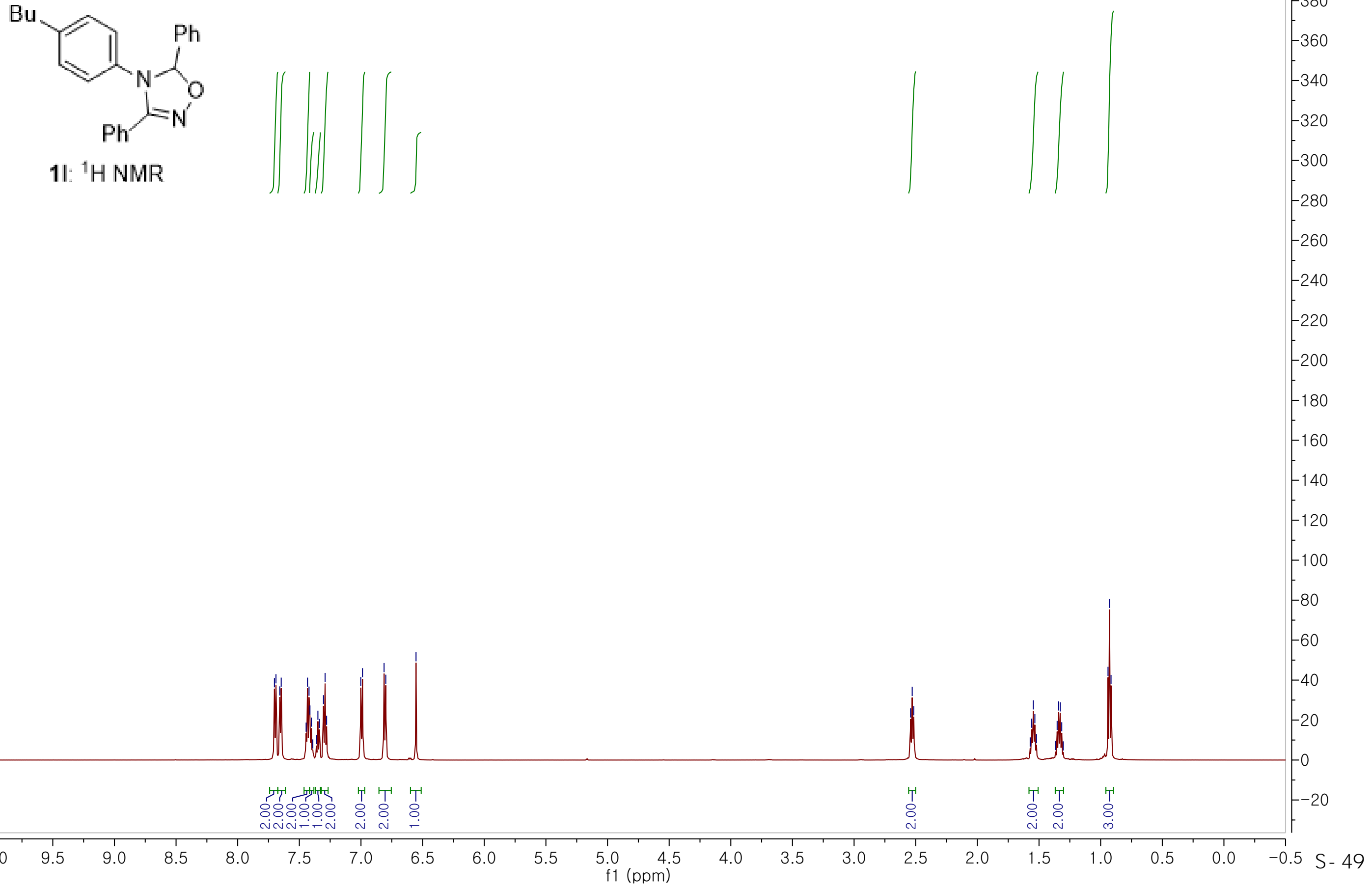


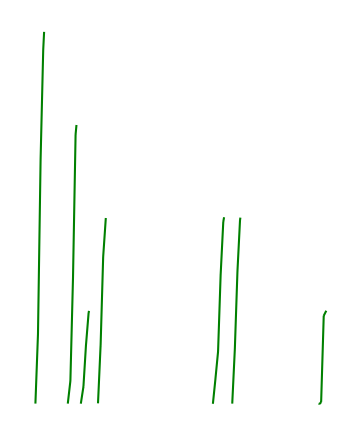<smiles>Fc1ccc(N2C(c3ccccc3)=NOC2c2ccccc2)cc1</smiles>

1m: ${ }^{1} \mathrm{H}$ NMR 
<smiles>Fc1ccc(N2C(c3ccccc3)=NOC2c2ccccc2)cc1</smiles>

$1 \mathrm{~m}:{ }^{13} \mathrm{C}$ NMR 


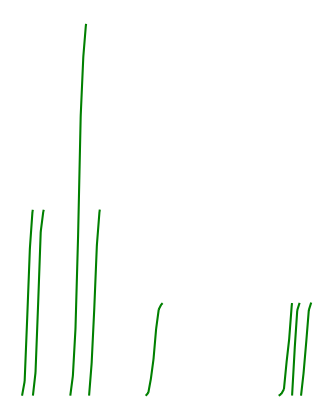<smiles>Fc1cc(N2C(c3ccccc3)=NOC2c2ccccc2)ccc1Cl</smiles>

1n: ${ }^{1}$ H NMR

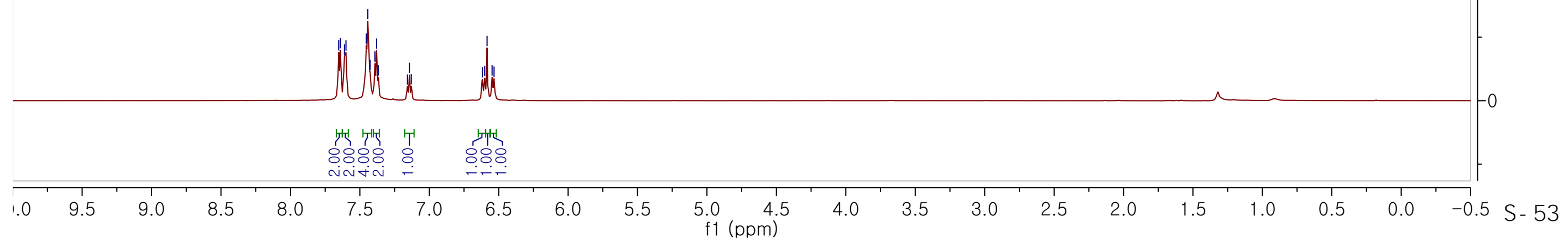



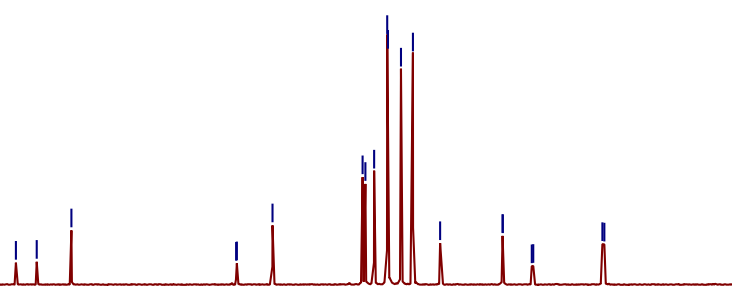

\section{1n: ${ }^{13} \mathrm{C}$ NMR}<smiles>[R]c1ccnc(/C=N\c2ccccc2)n1</smiles> 

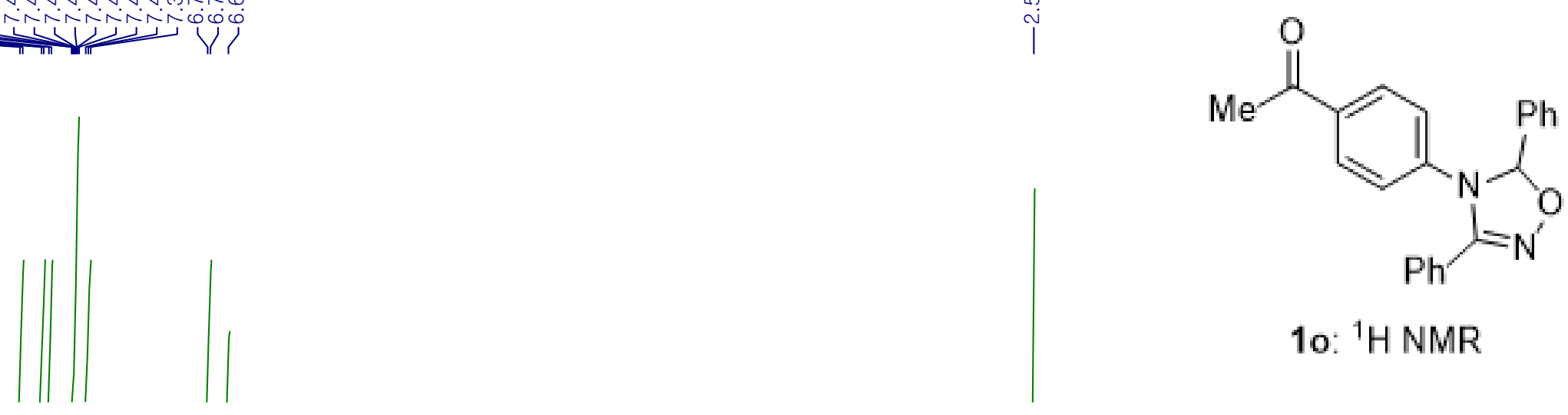

10: ${ }^{1} \mathrm{H}$ NMR

ind

Ii

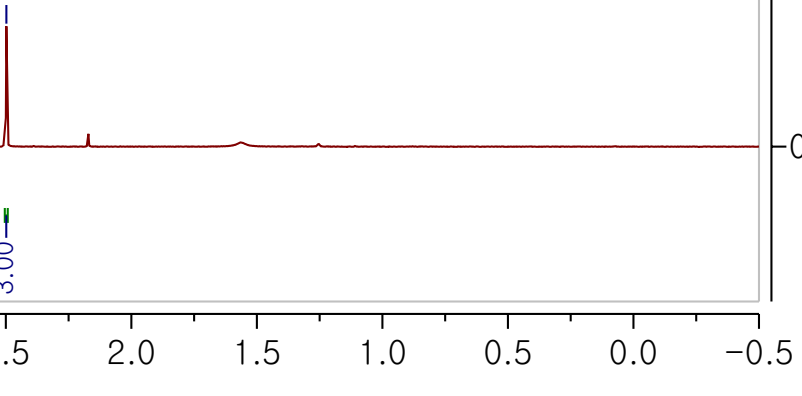

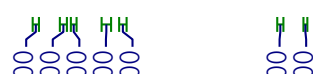

1
7.5




(1)

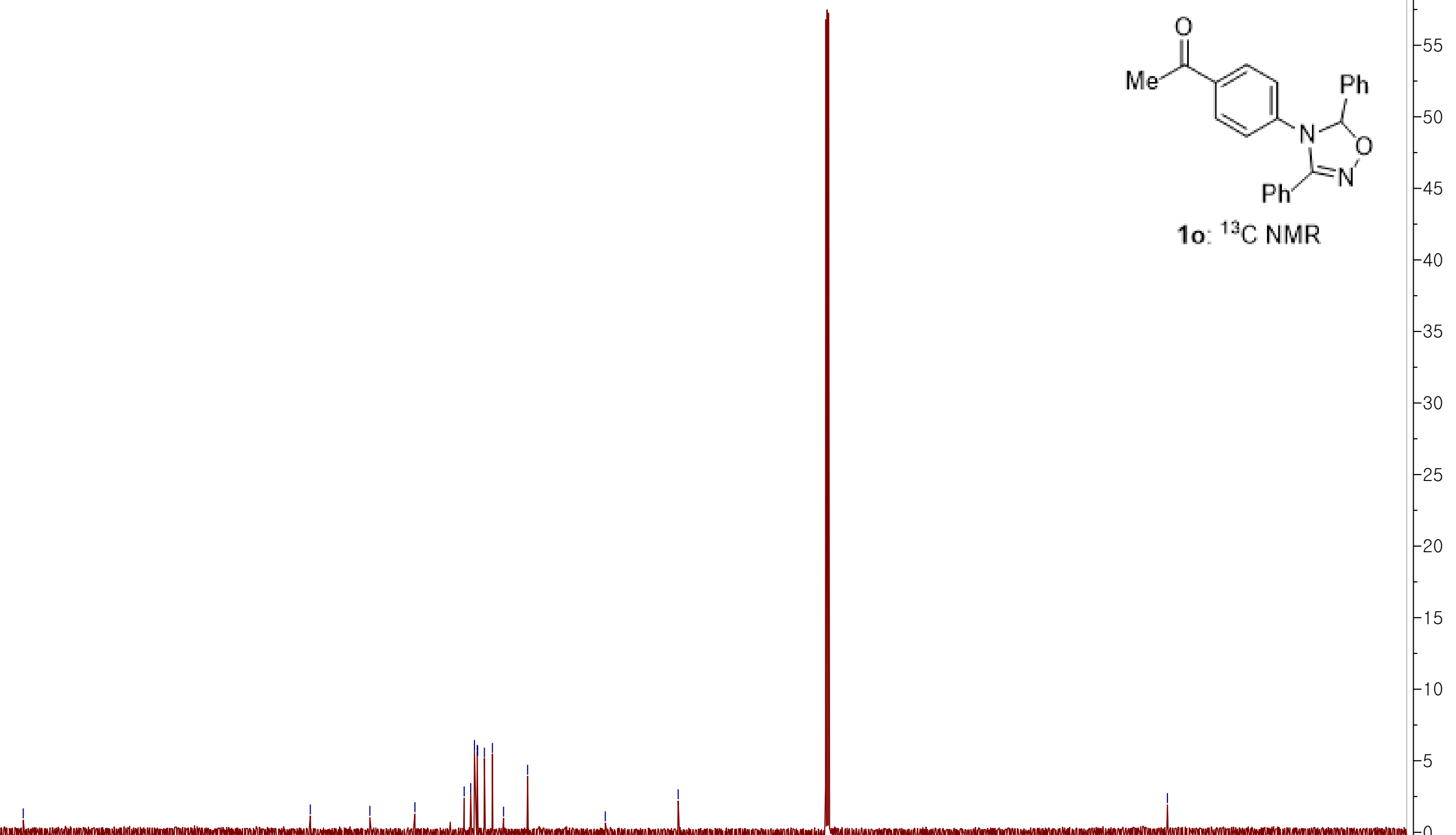

Mnom 


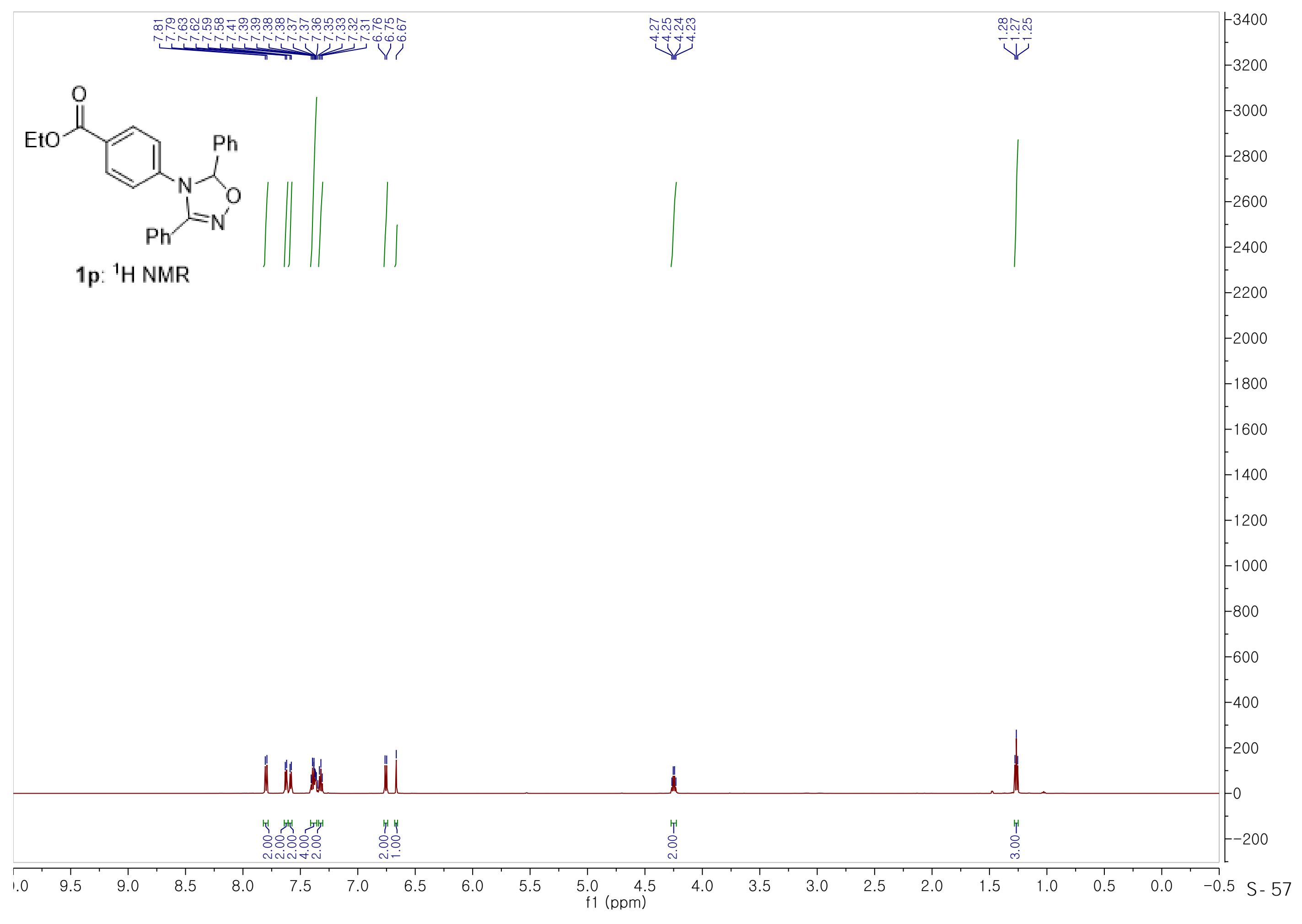




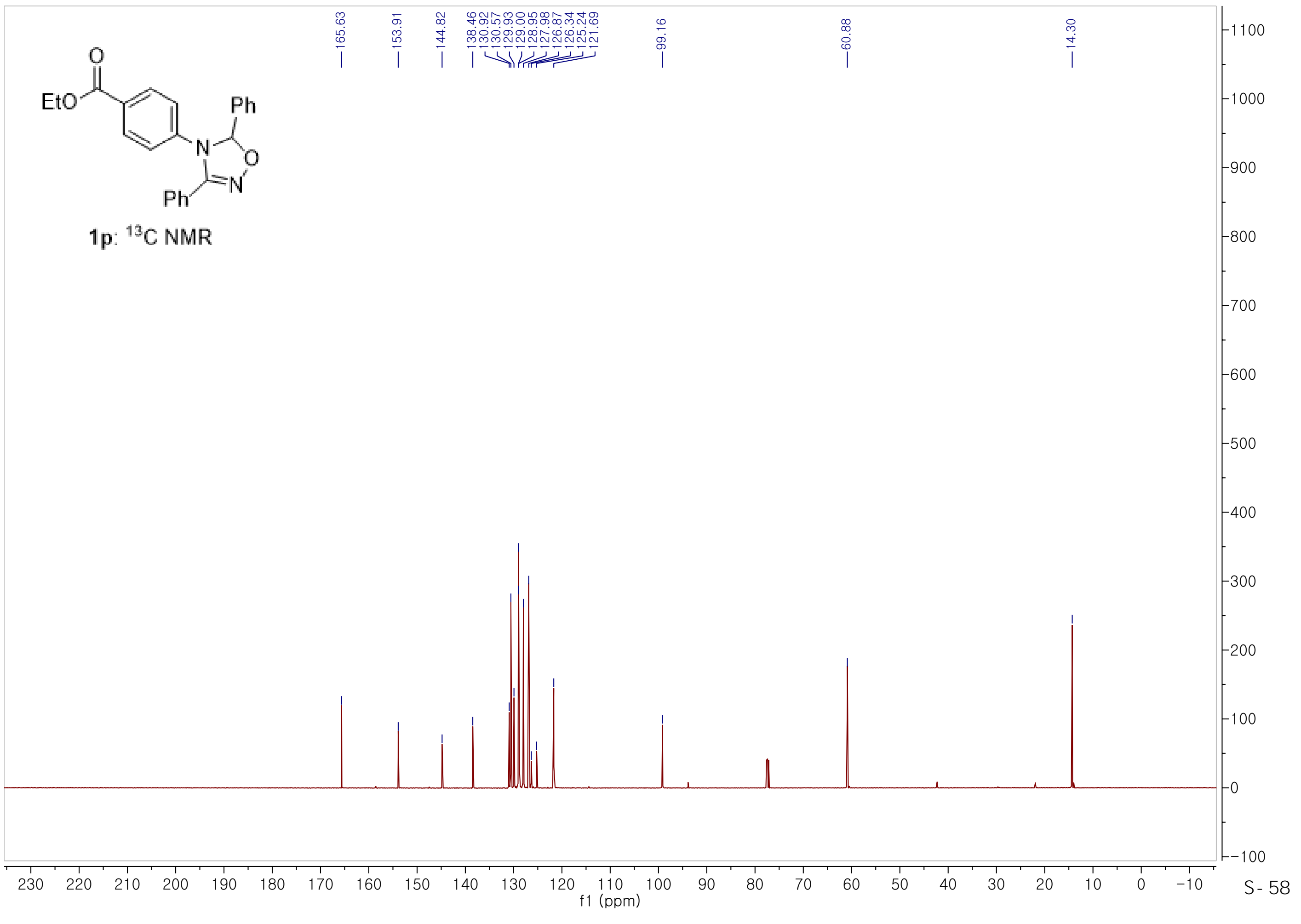




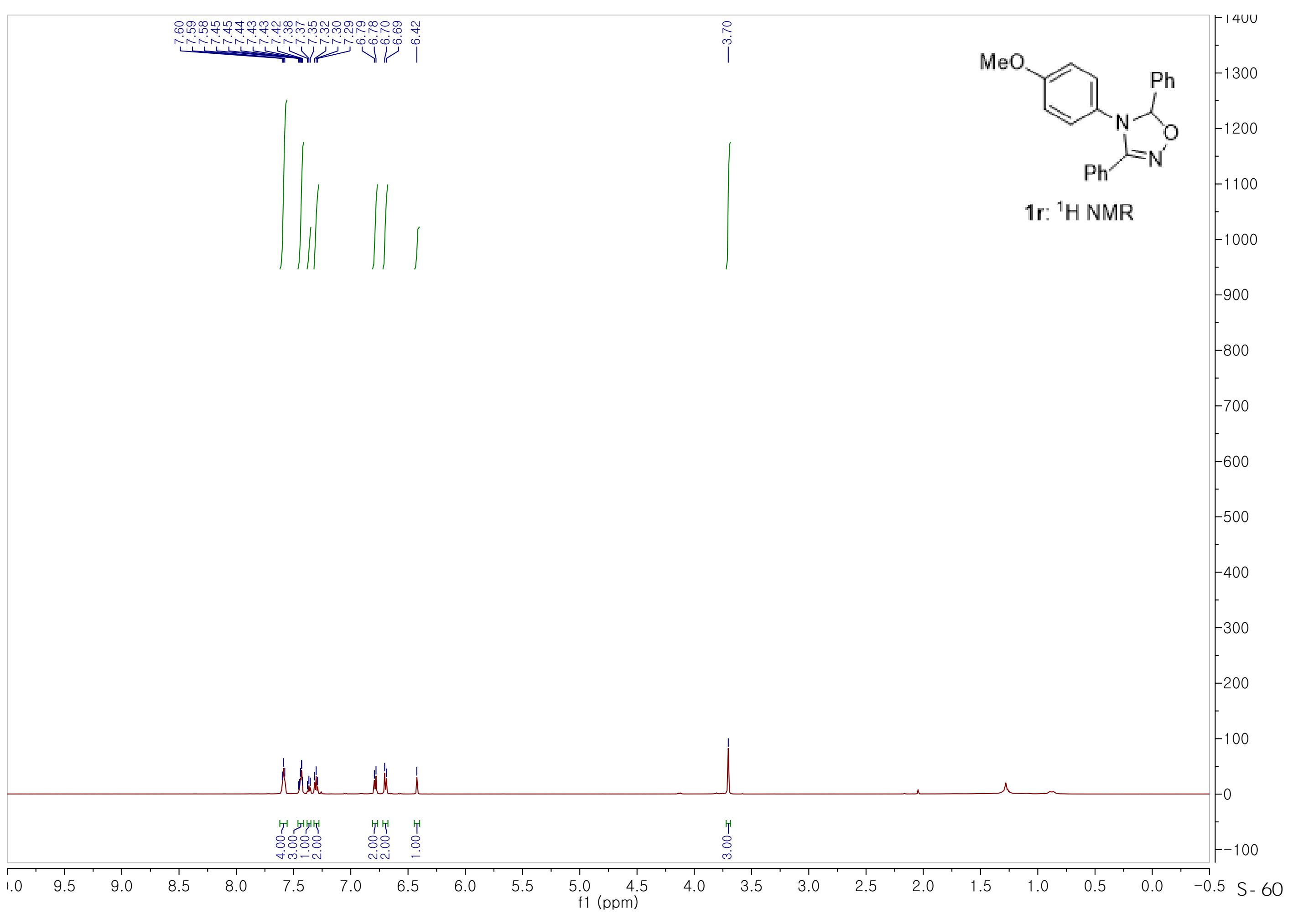


$\mathrm{MeO}$ 


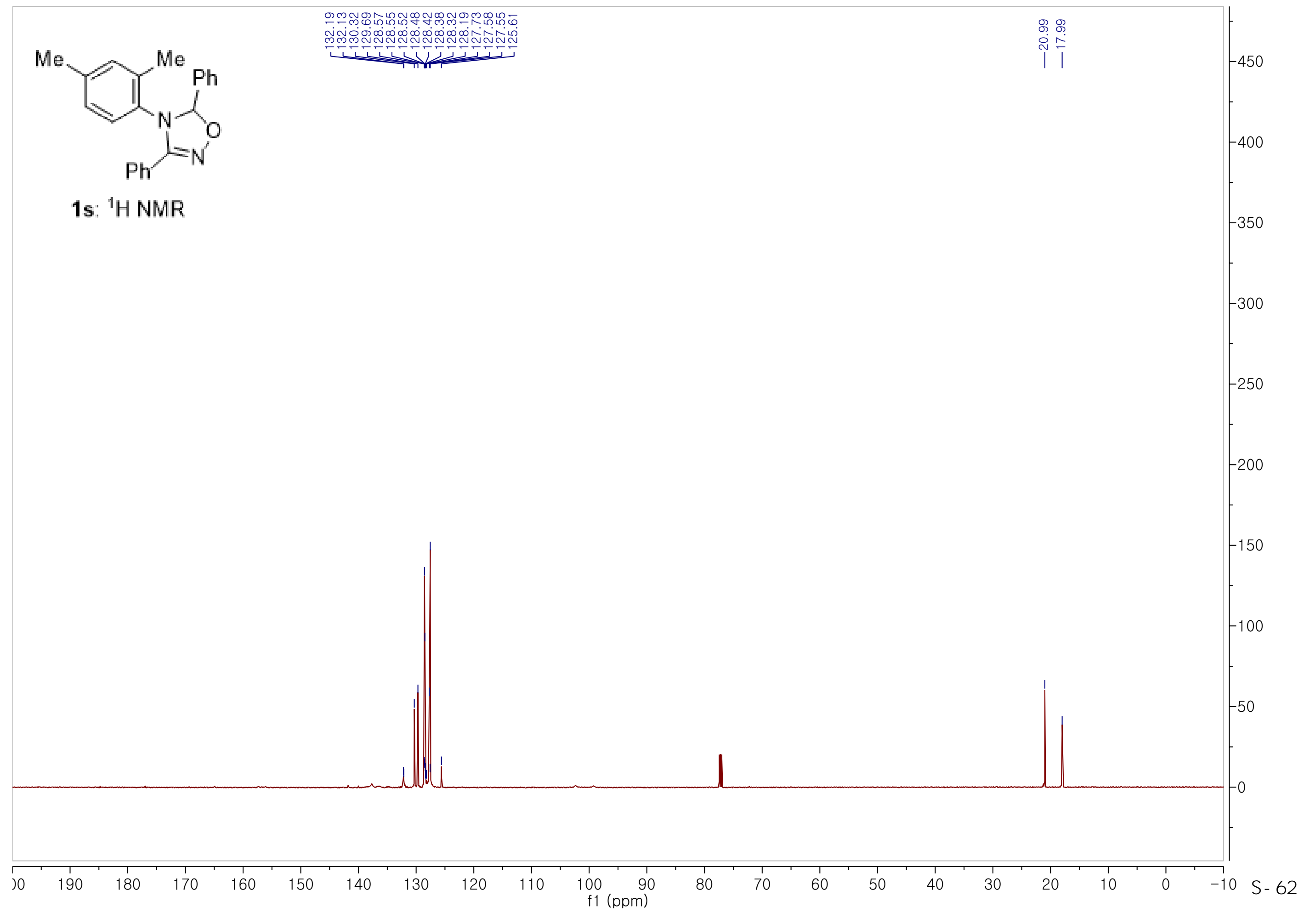




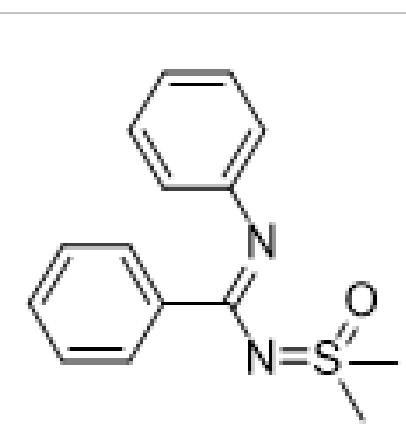

2a: ${ }^{1} \mathrm{H}$ NMR
$-260$

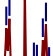
州

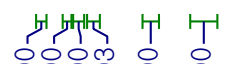
1,10

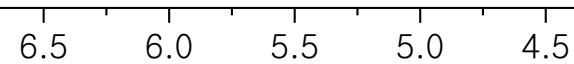
f1 (ppm) 


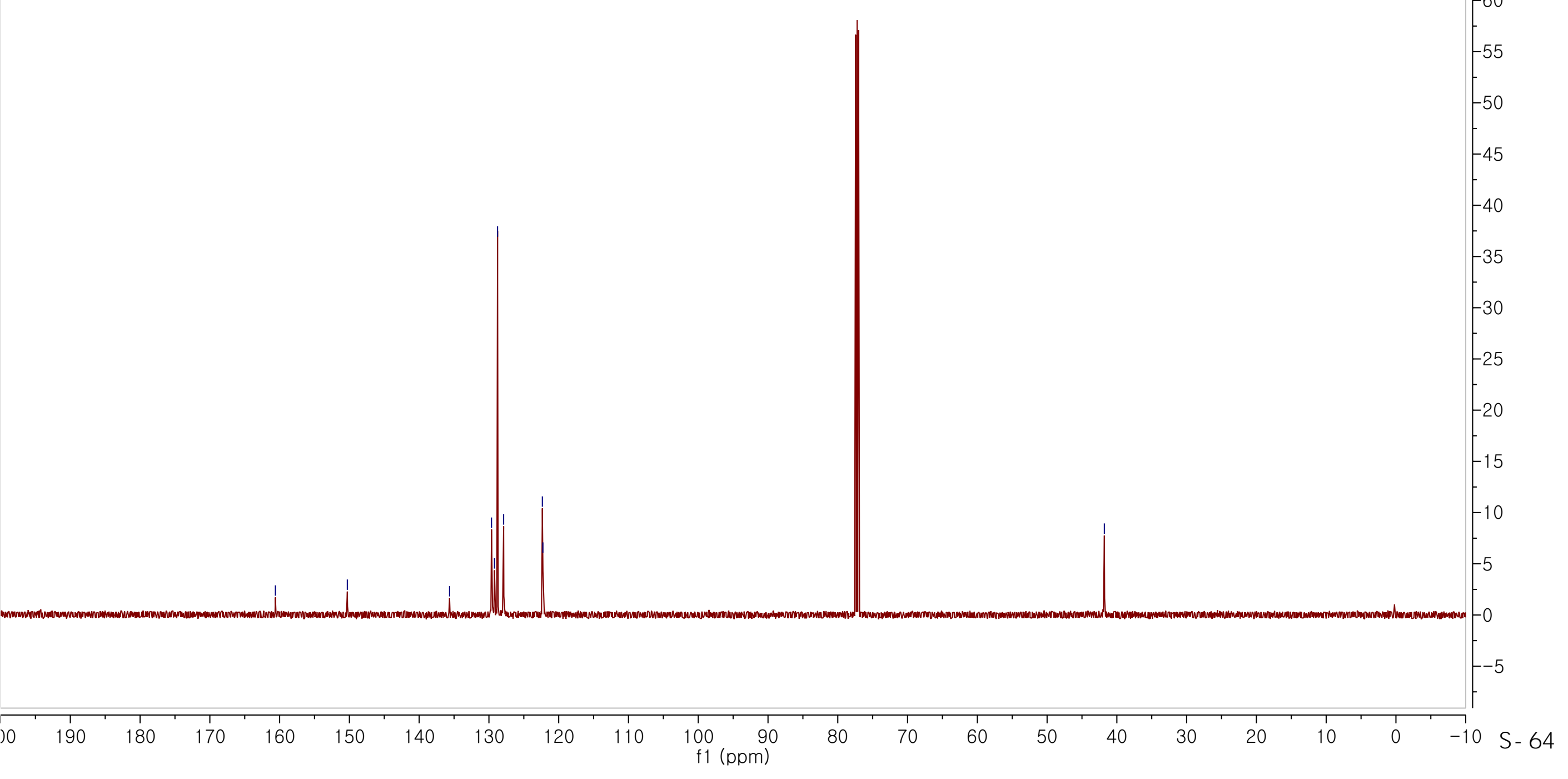




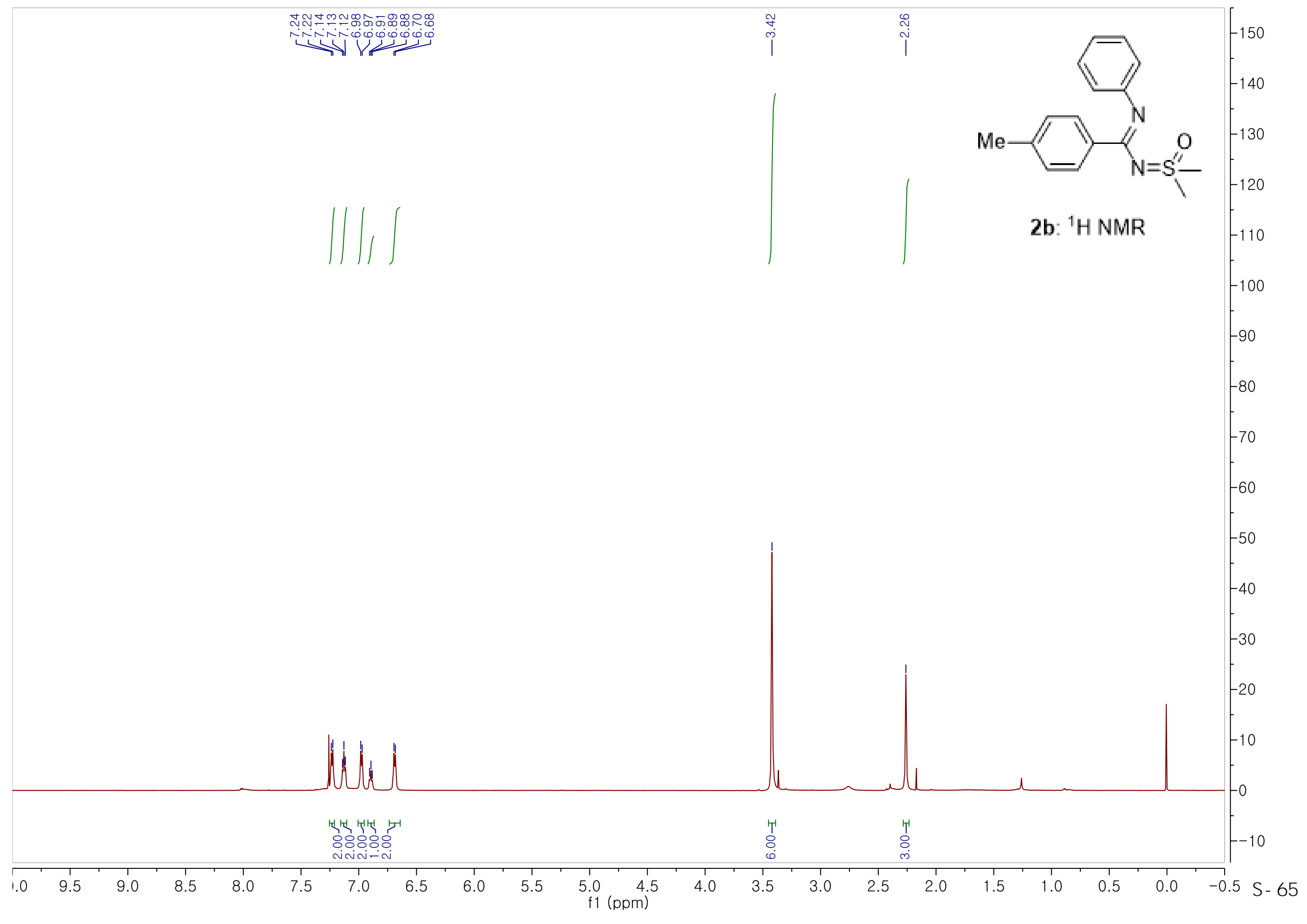




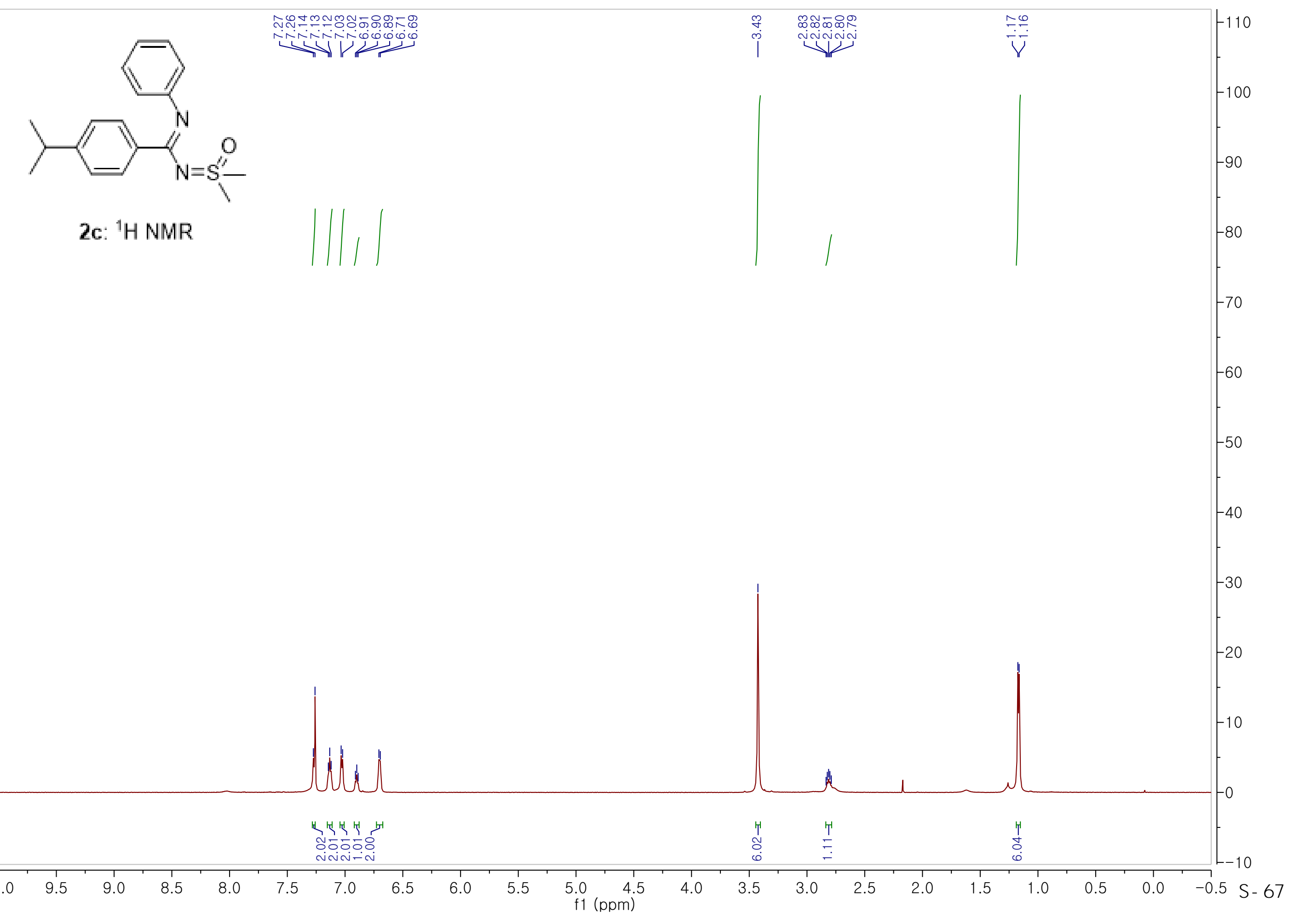



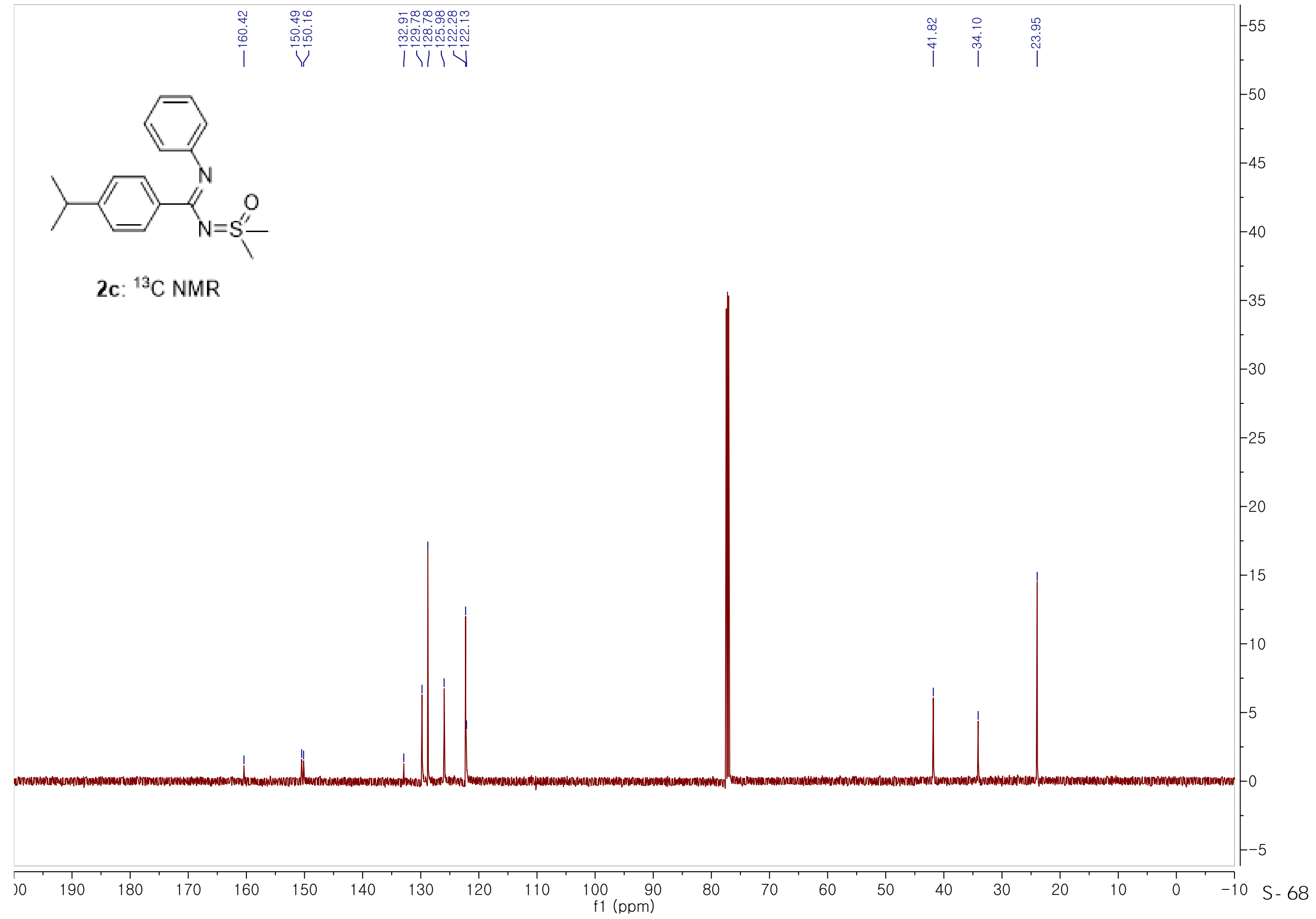


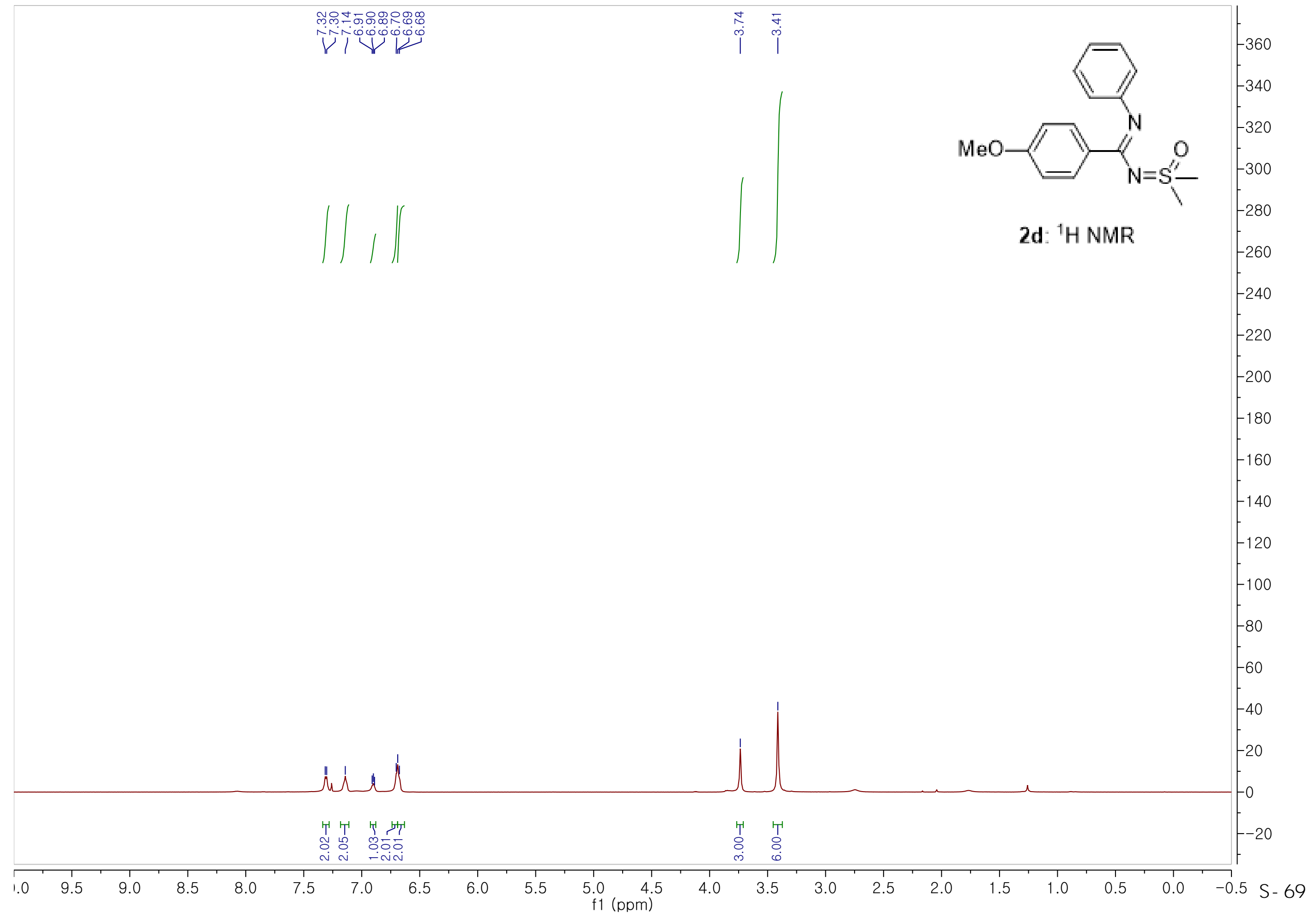




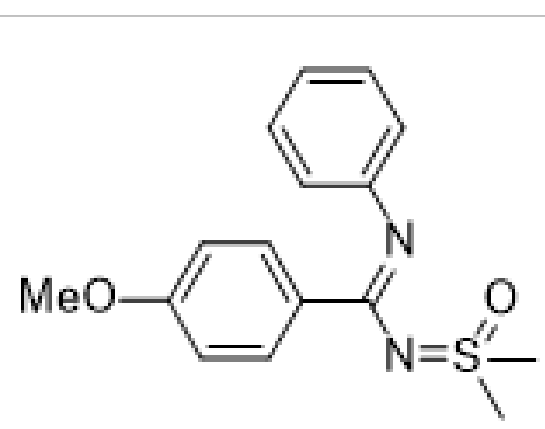

2d: ${ }^{13} \mathrm{C}$ NMR

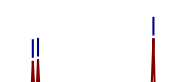




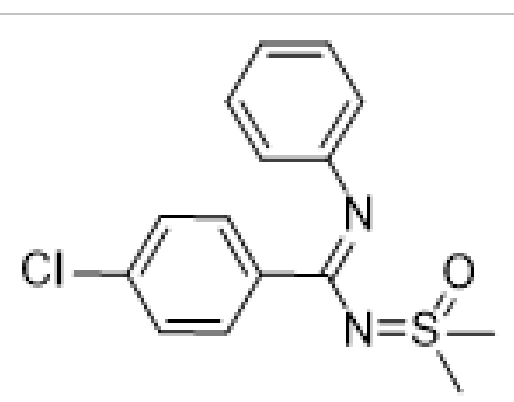

$-85$

2e: ${ }^{1}$ H NMR 


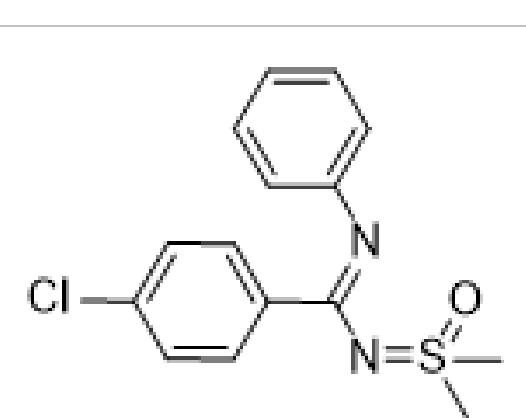




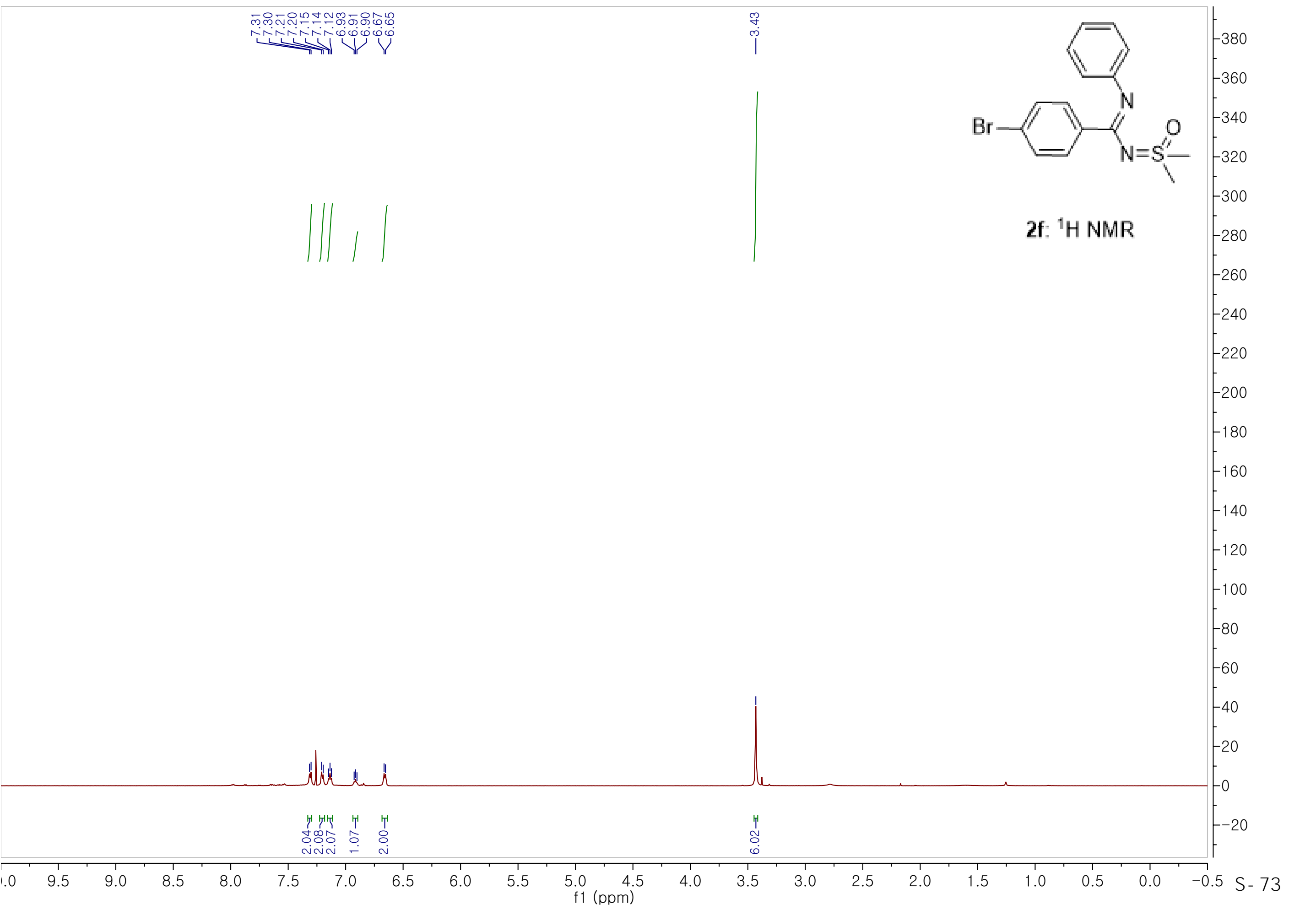




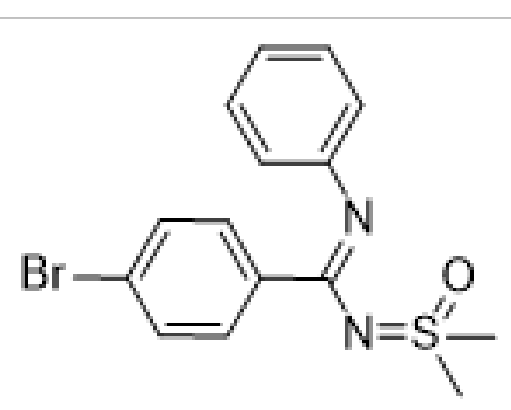

2f: ${ }^{13} \mathrm{C}$ NMR 

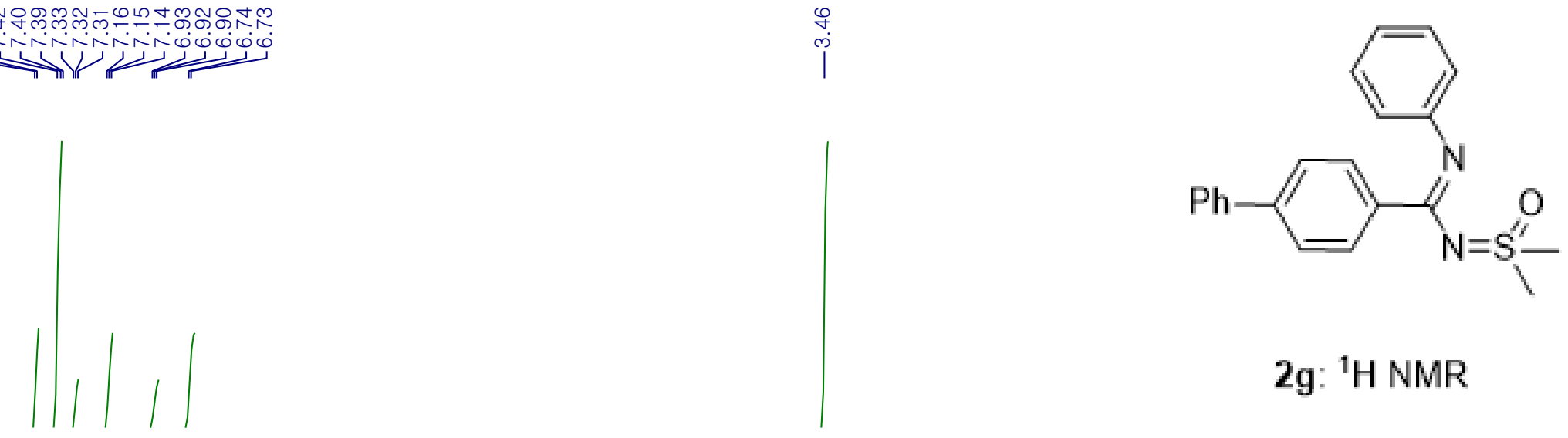

2g: ${ }^{1} \mathrm{H}$ NMR

Whet

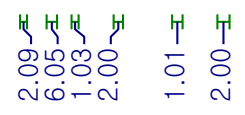
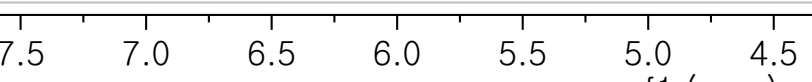


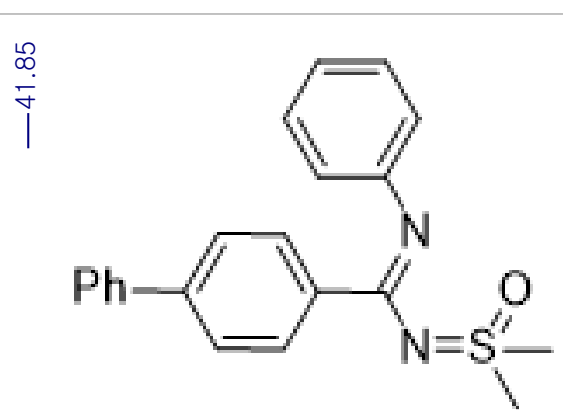

2g: ${ }^{13} \mathrm{C}$ NMR 


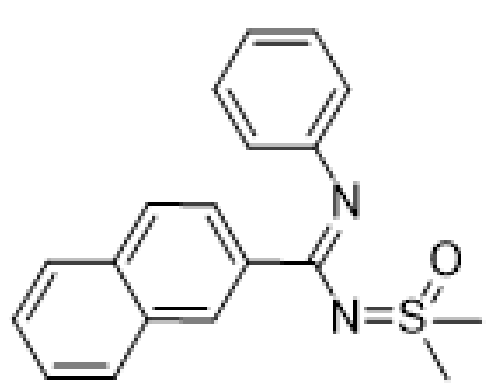

2h: ${ }^{1}$ H NMR

$\ln |d|$

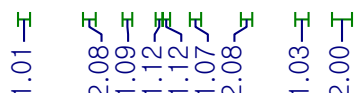

$\stackrel{1}{8}$ 


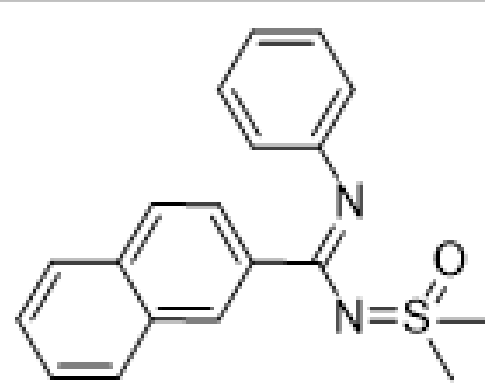

2h: ${ }^{13} \mathrm{C}$ NMR 


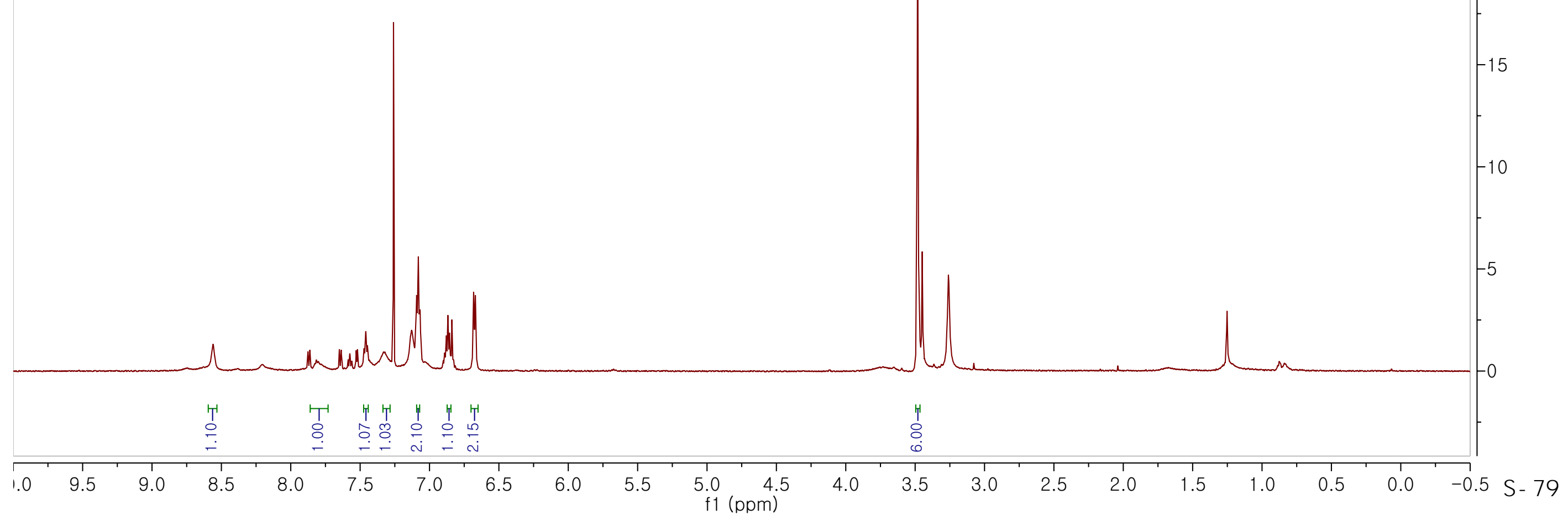



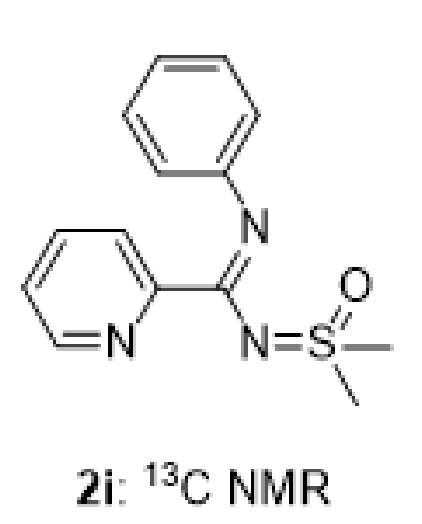


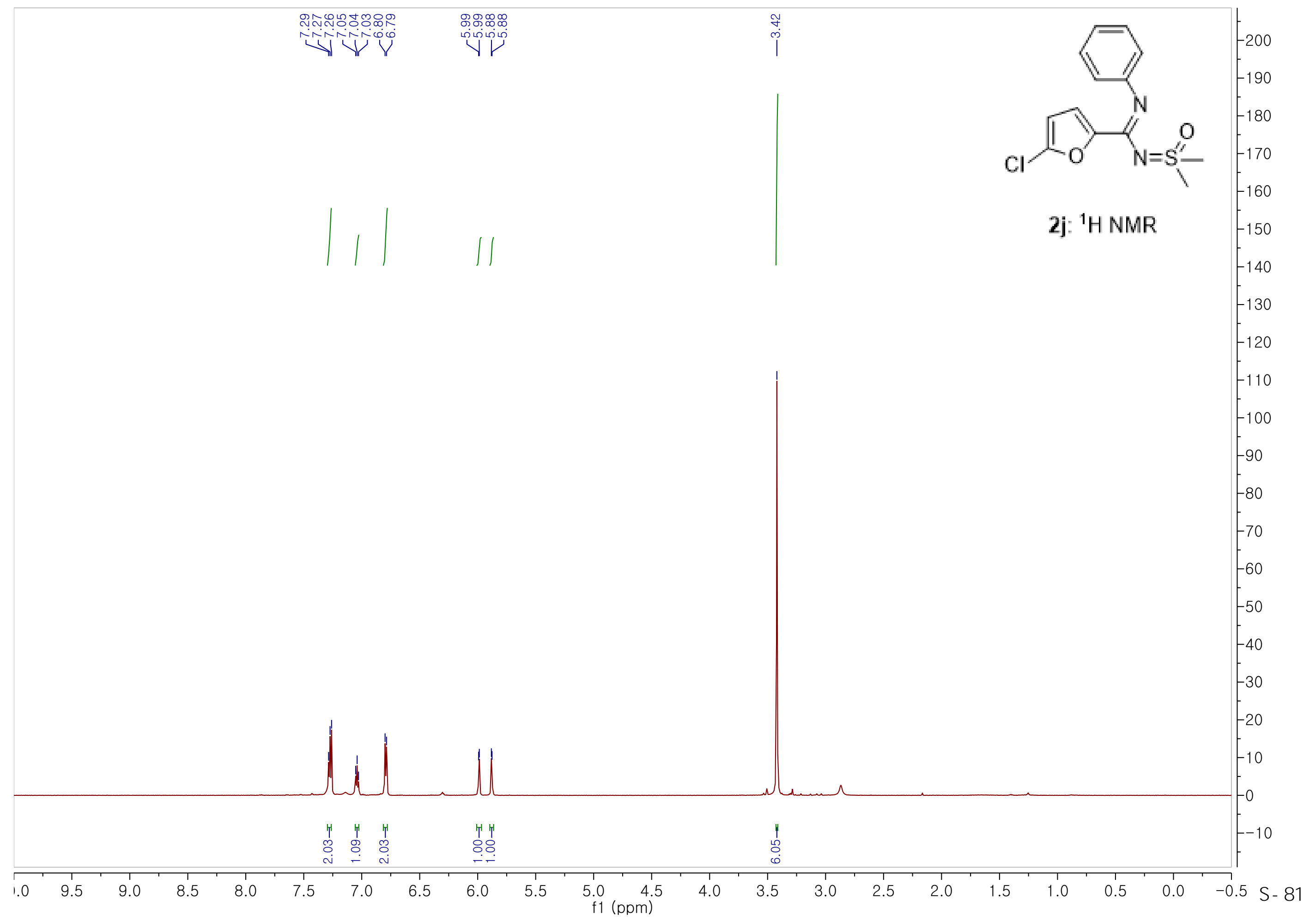




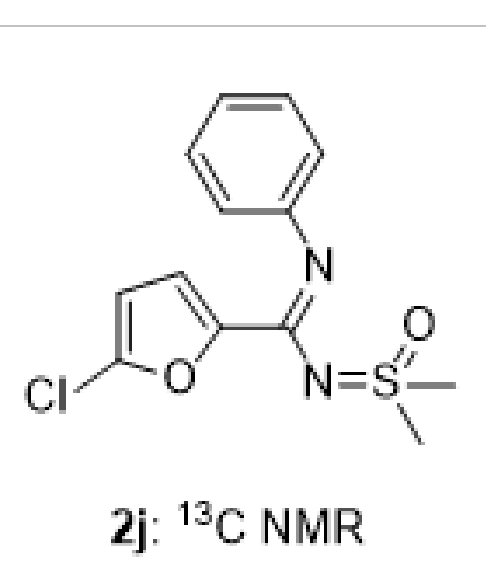




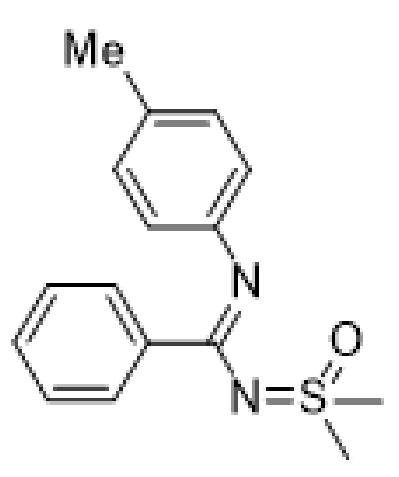

2k: ${ }^{1} \mathrm{H}$ NMR

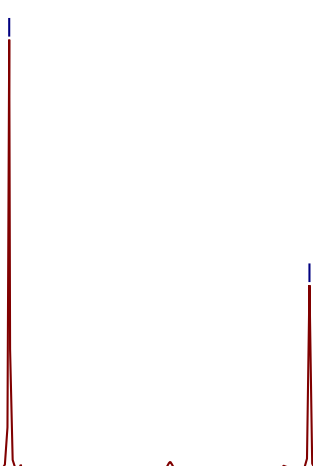




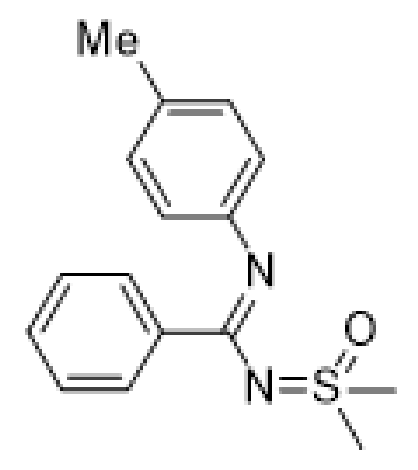

2k. ${ }^{13} \mathrm{C}$ NMR 


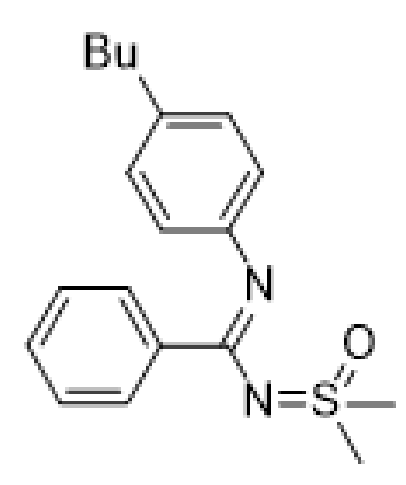

2l: ${ }^{1} \mathrm{H}$ NMR

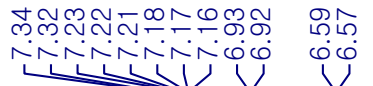

$\underbrace{1}$ 


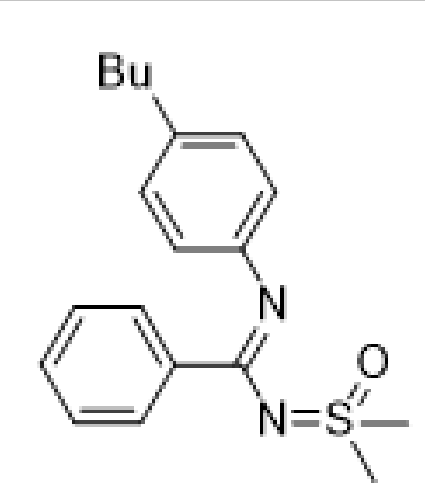

กำ

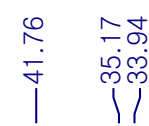

2l: ${ }^{13} \mathrm{C}$ NMR 


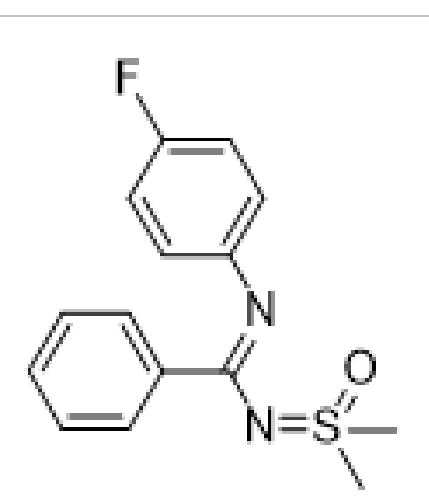

$-160$

2m: ${ }^{1} H$ NMR 


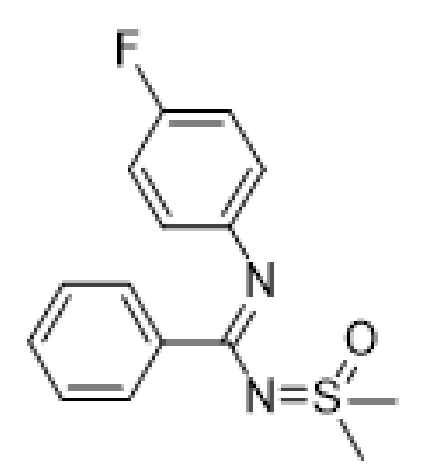

$2 \mathrm{~m}:{ }^{13} \mathrm{C}$ NMR 

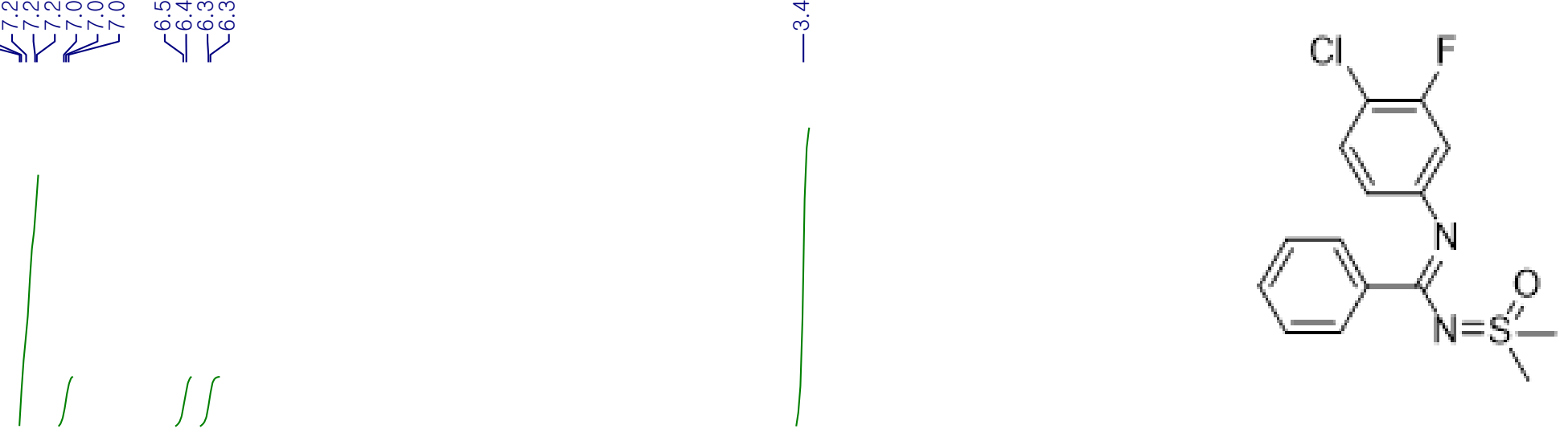

2n: ${ }^{1}$ H NMR

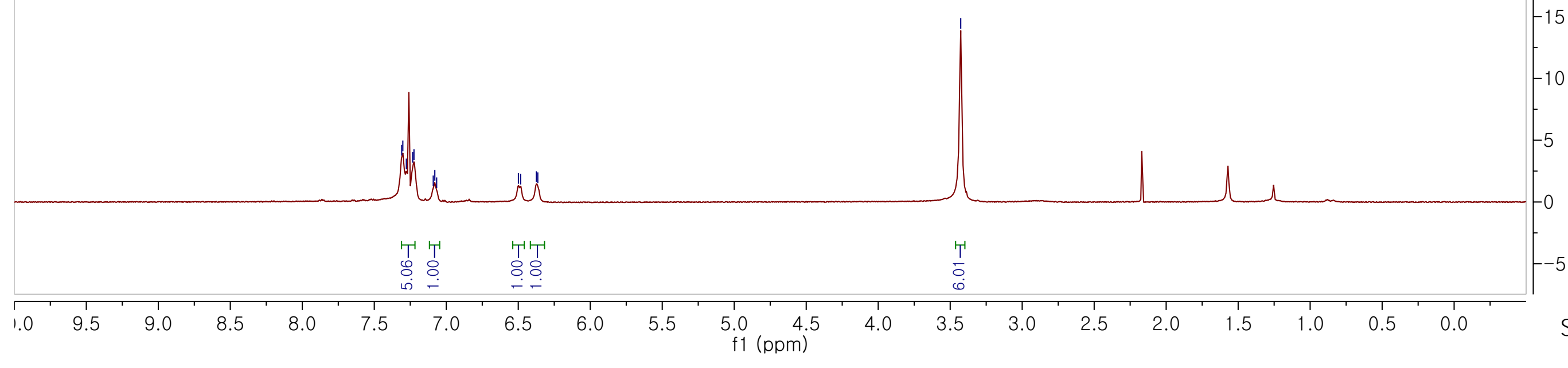




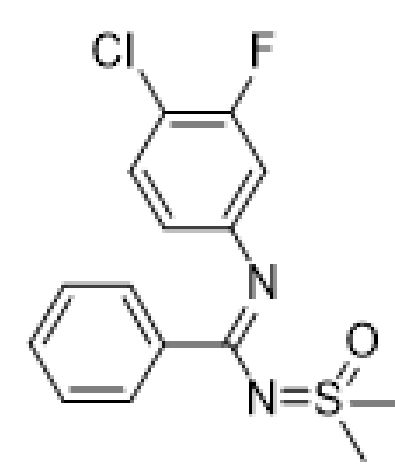

2n: ${ }^{13} \mathrm{C}$ NMR 


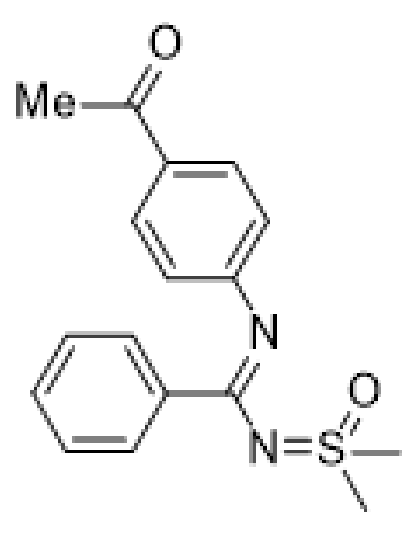

2o: ${ }^{1} \mathrm{H}$ NMR

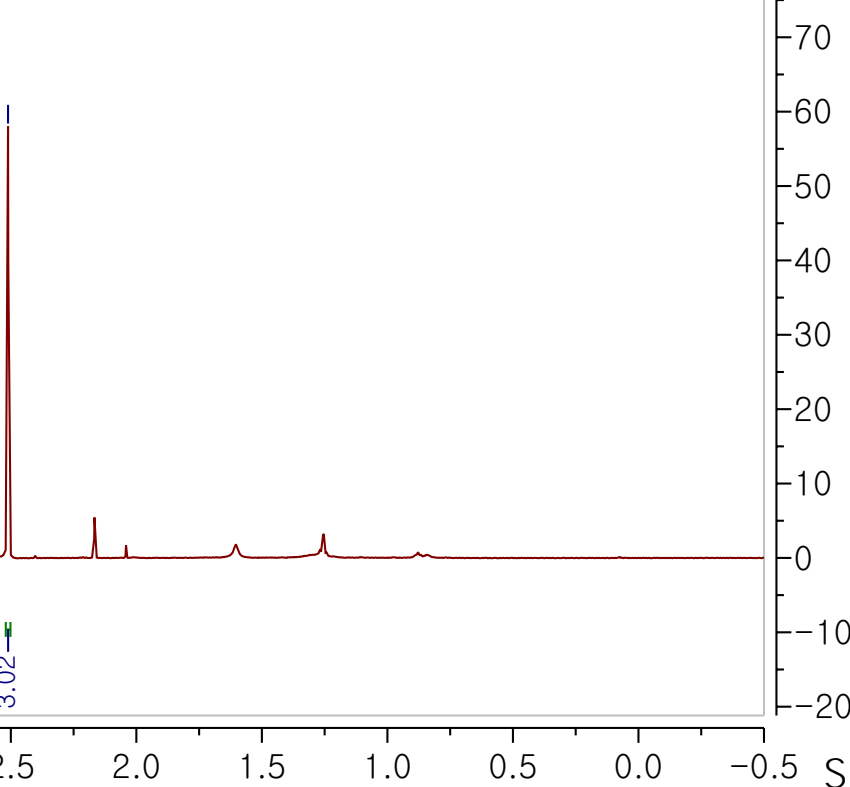


2o: ${ }^{13} \mathrm{C}$ NMR

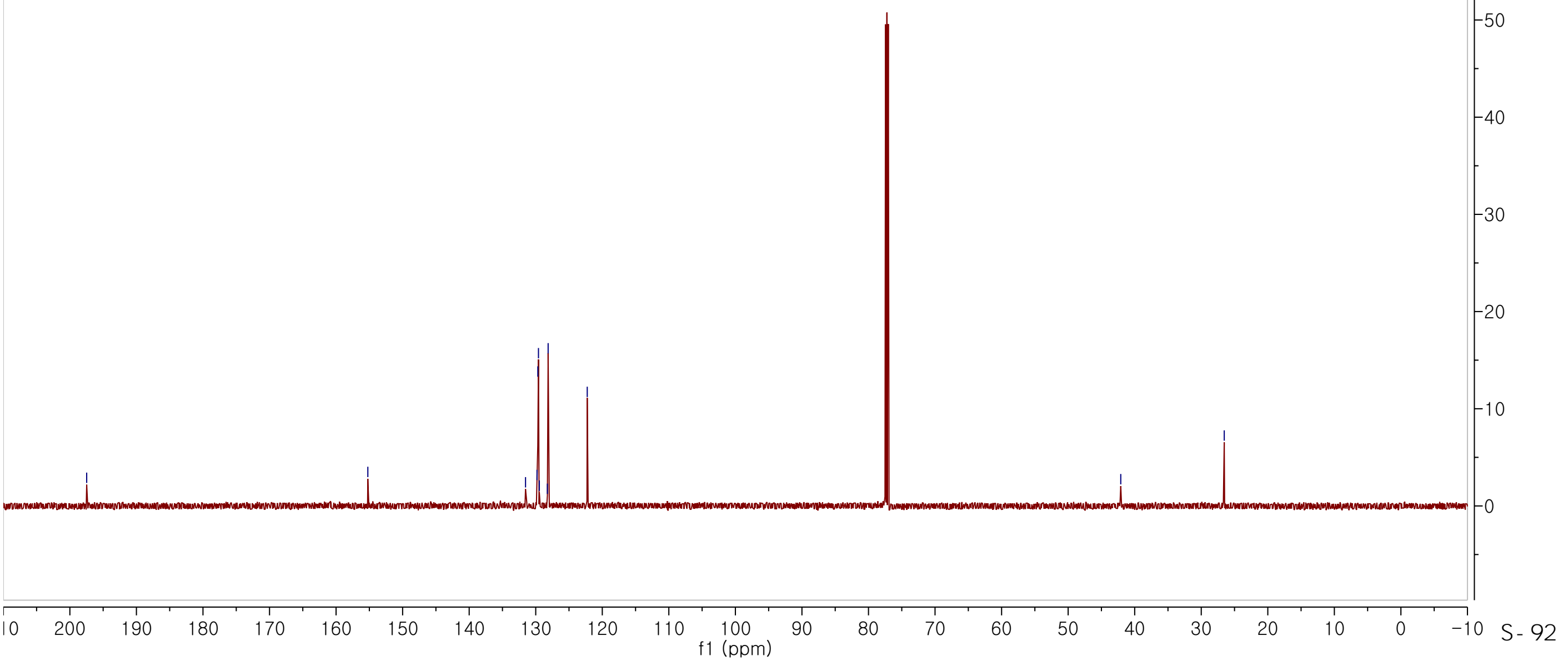




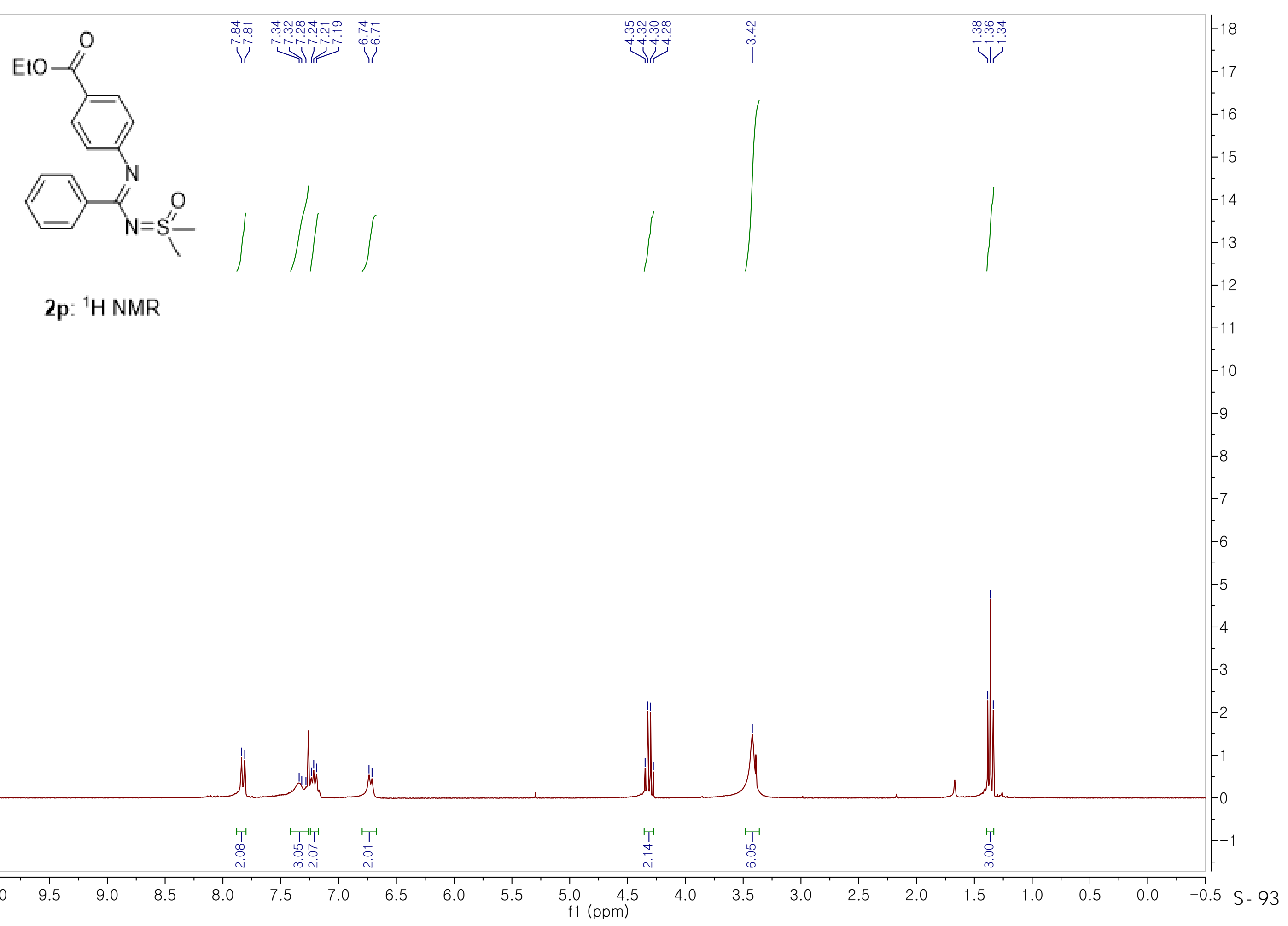




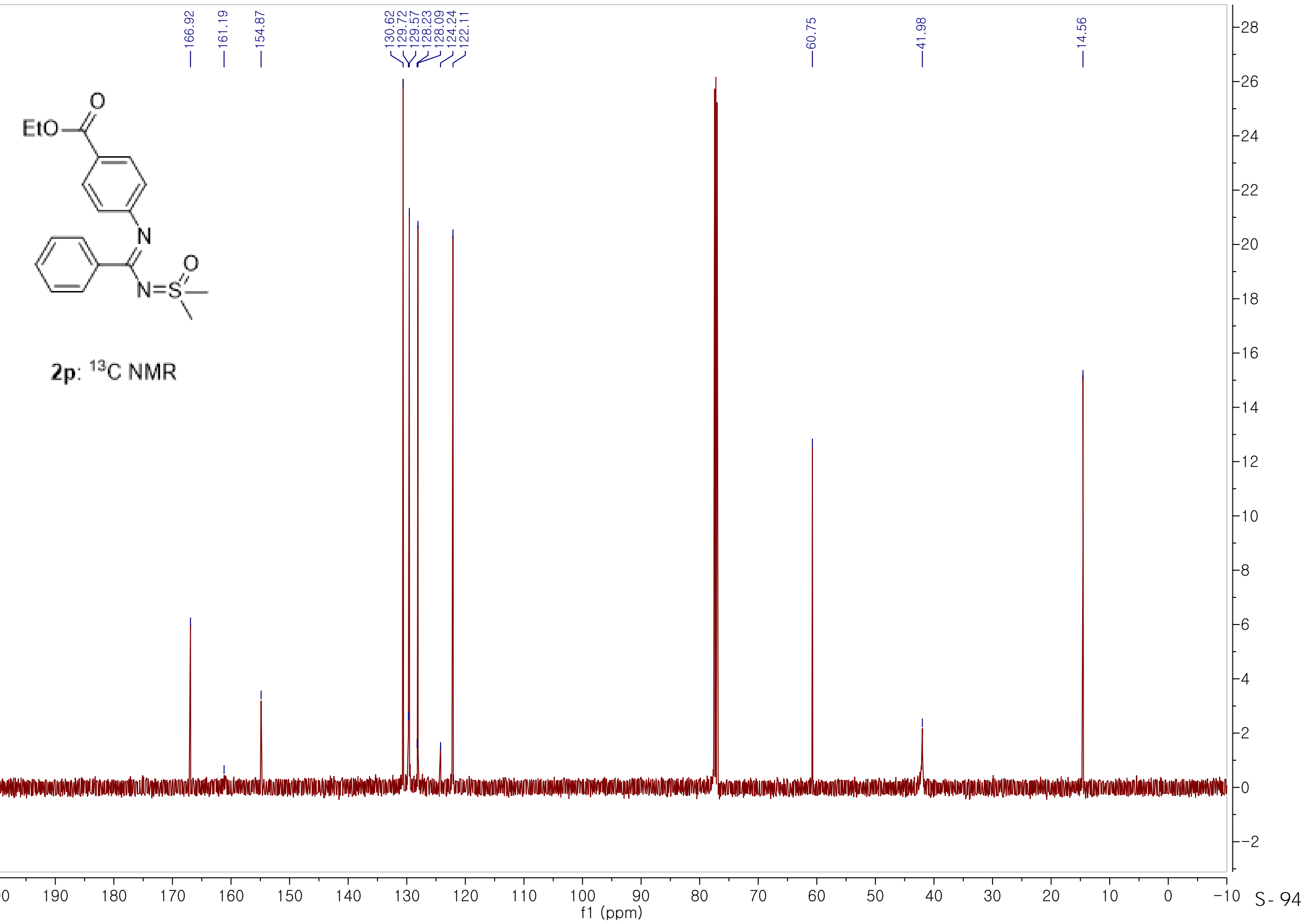




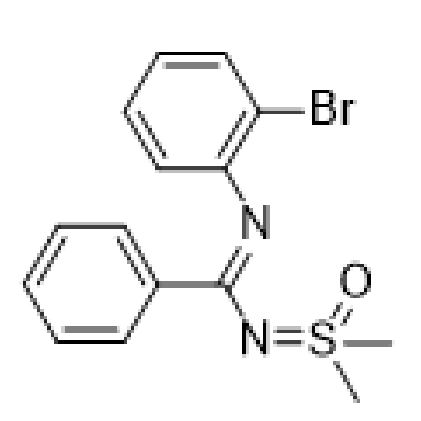

$-65$

2q: ${ }^{1} \mathrm{H}$ NMR 


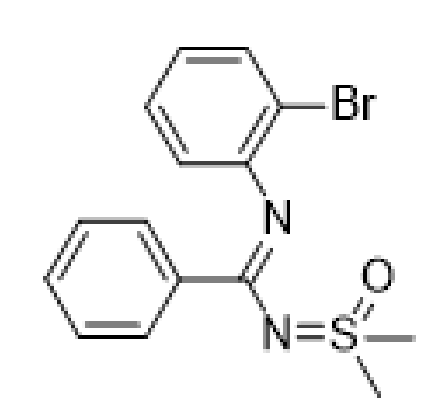

2q: ${ }^{13}$ C NMR 


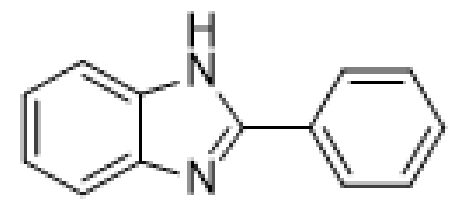

$-650$

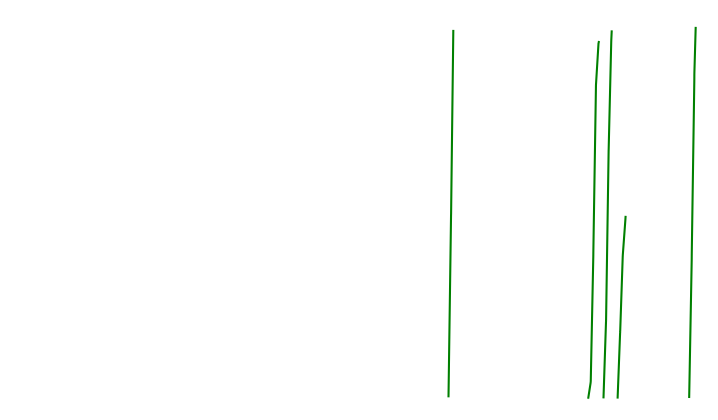

3a: ${ }^{1} \mathrm{H}$ NMR

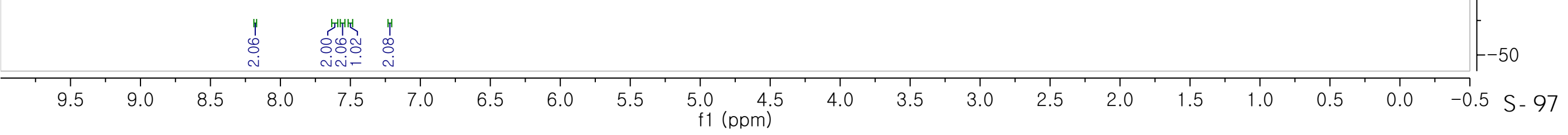




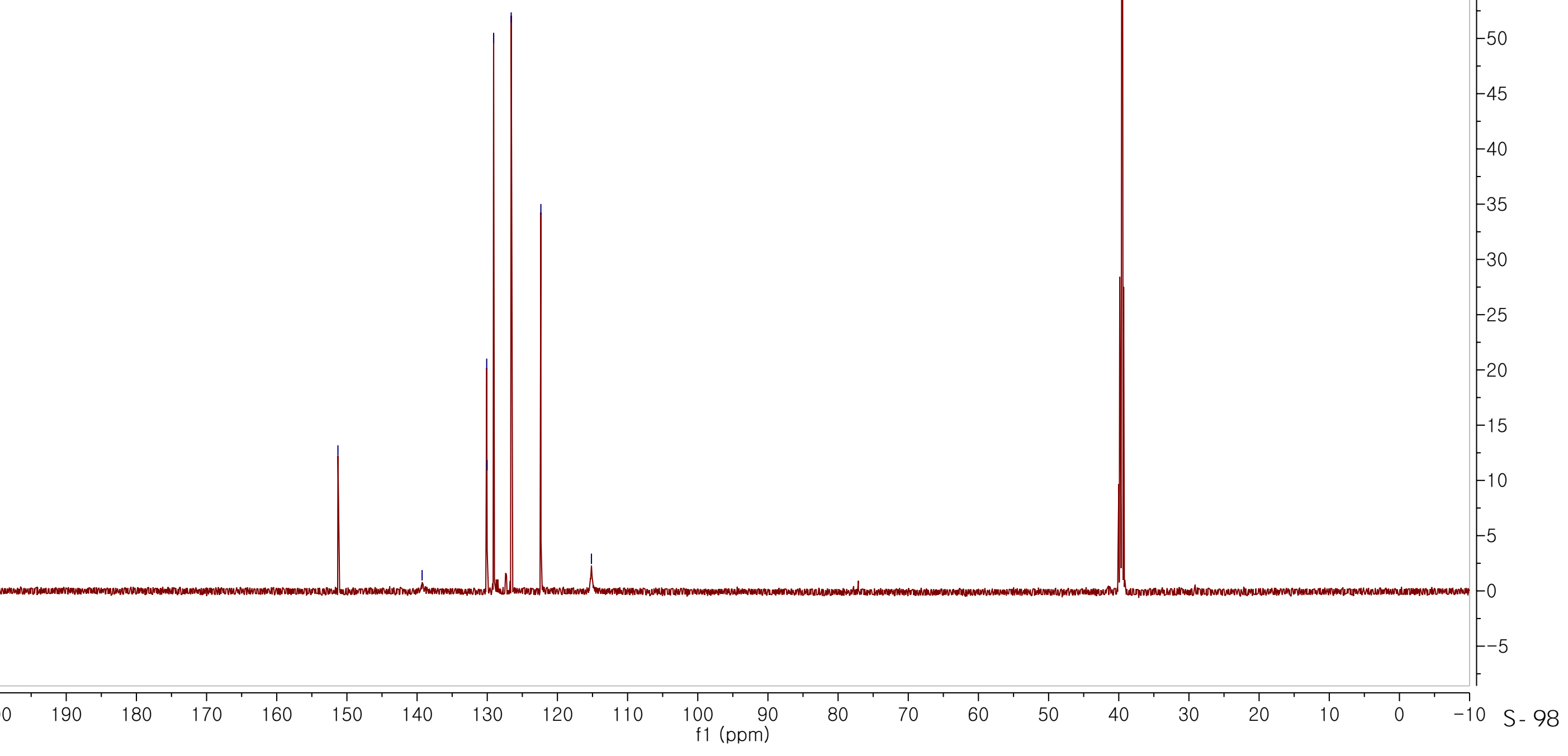




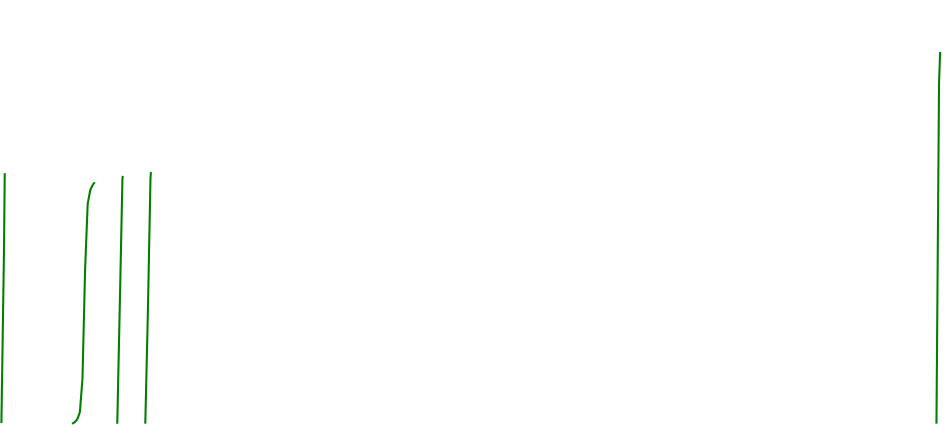<smiles>Cc1ccc(-c2nc3ccccc3[nH]2)cc1</smiles>

3b: ${ }^{1}$ H NMR 


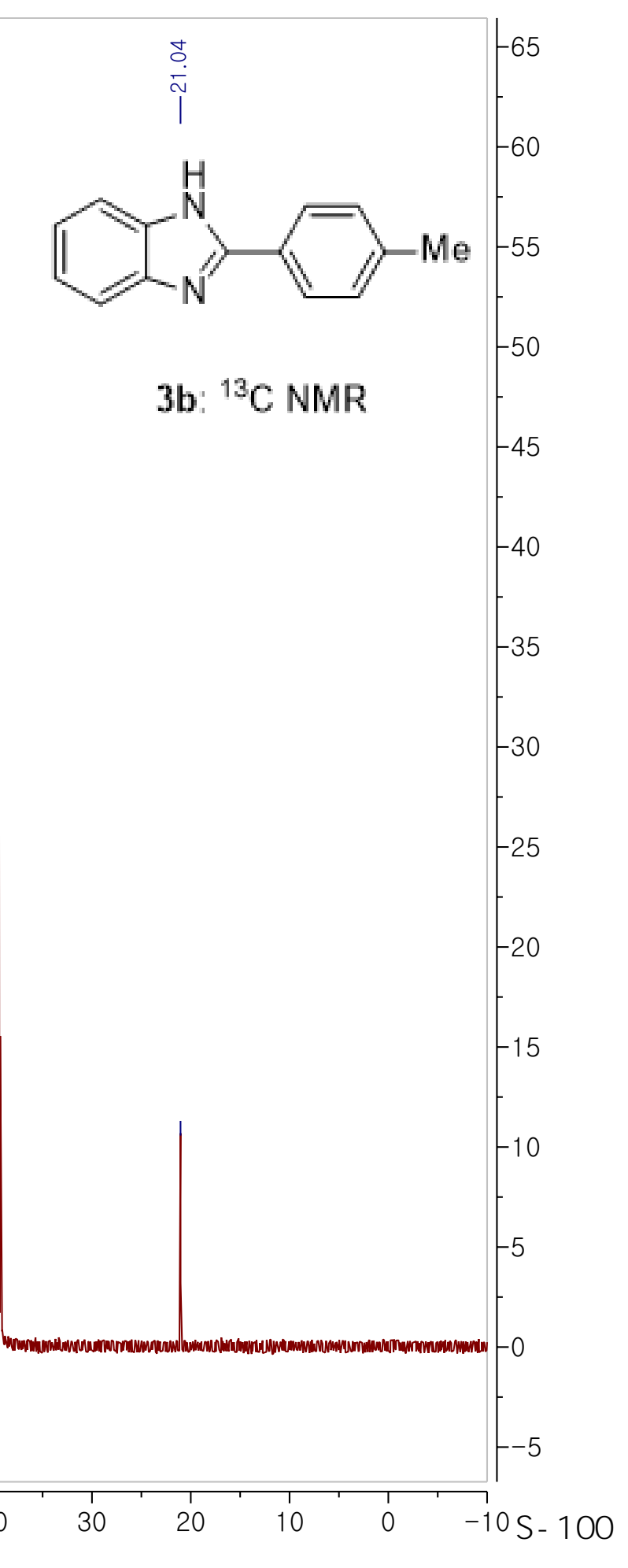




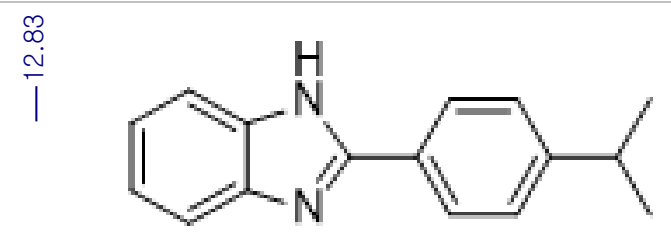

\section{3c: ${ }^{1} \mathrm{H}$ NMR}
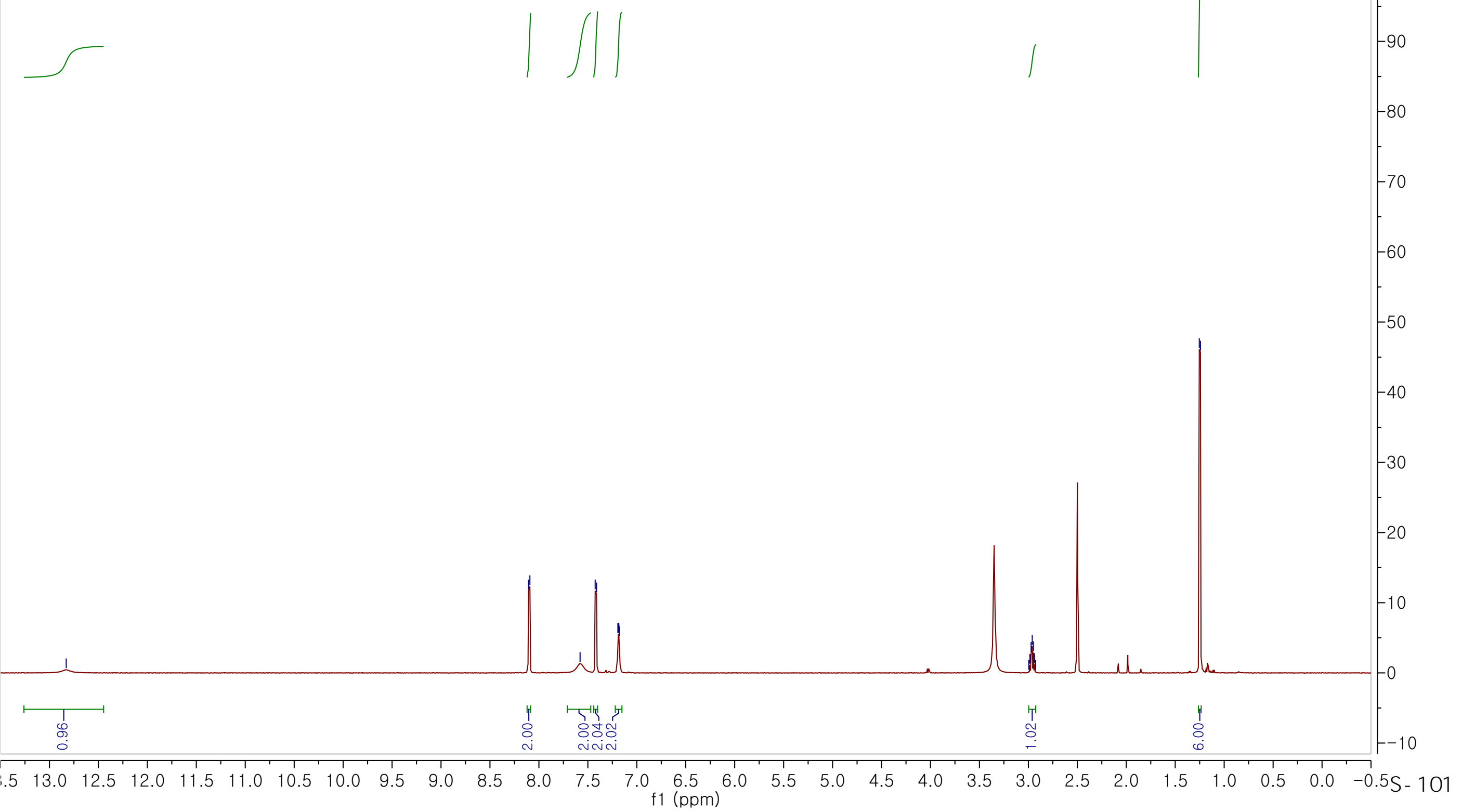


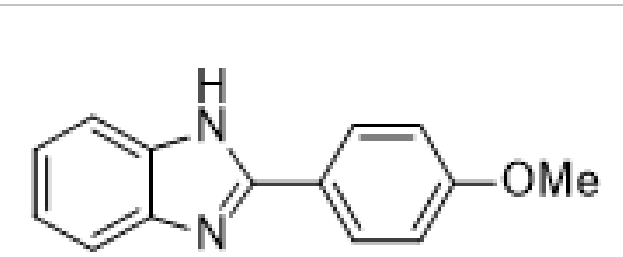

3d: ${ }^{1} \mathrm{H}$ NMR 


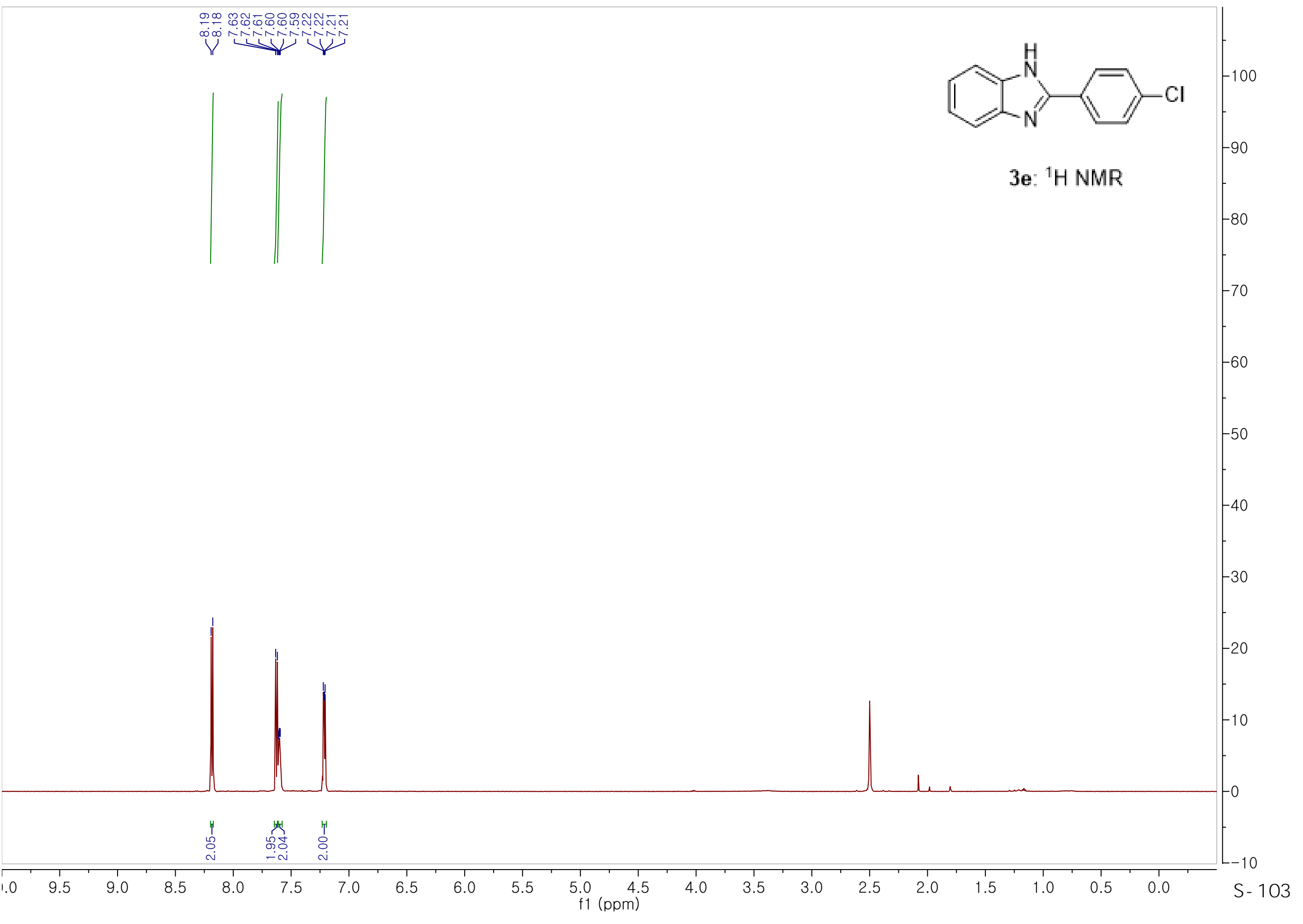




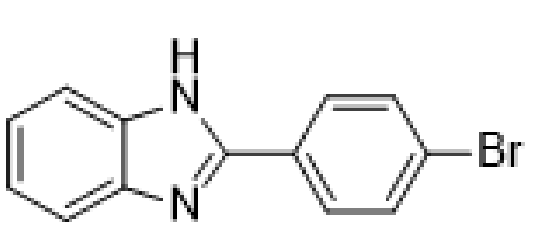

3f: ${ }^{1} \mathrm{H}$ NMR

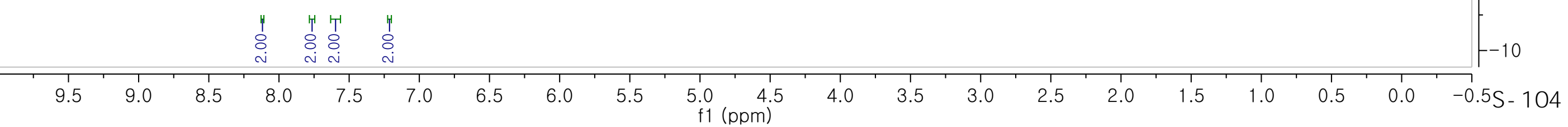




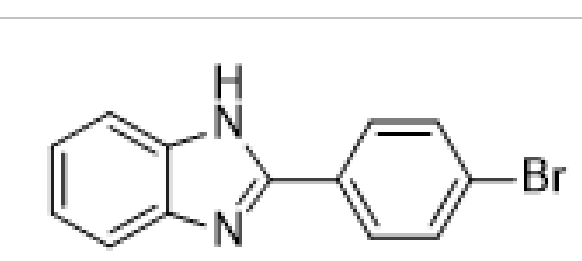

3f: ${ }^{13} \mathrm{C}$ NMR

$1-45$ 


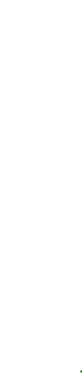

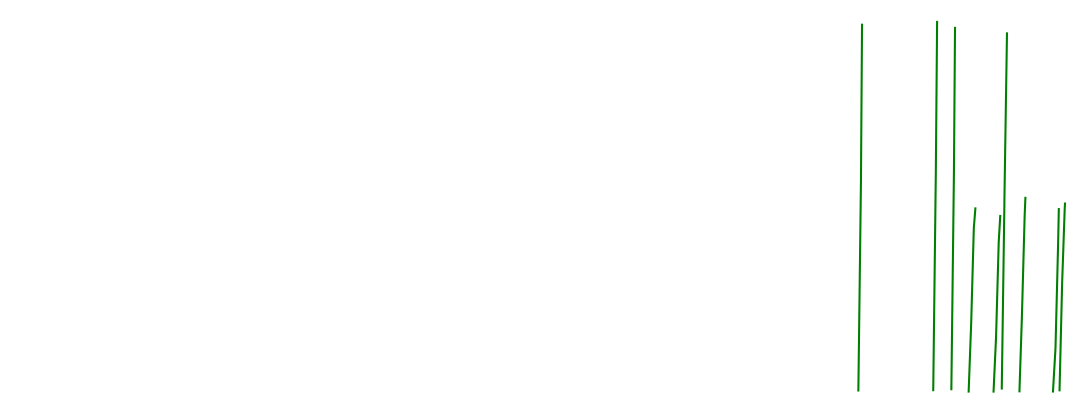

3g: ${ }^{1}$ H NMR

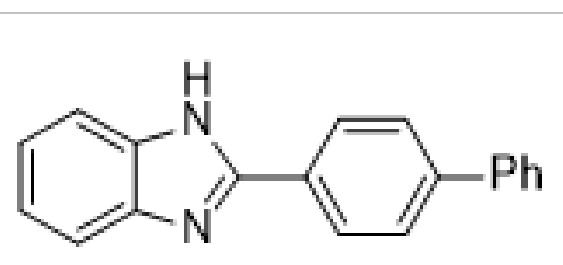




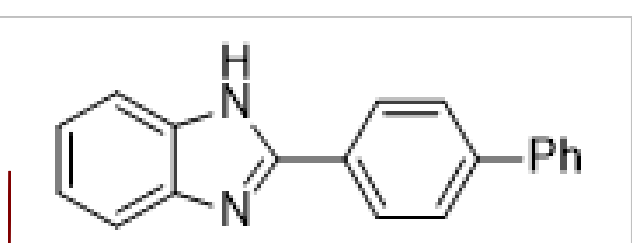

3g: ${ }^{13} \mathrm{C}$ NMR 


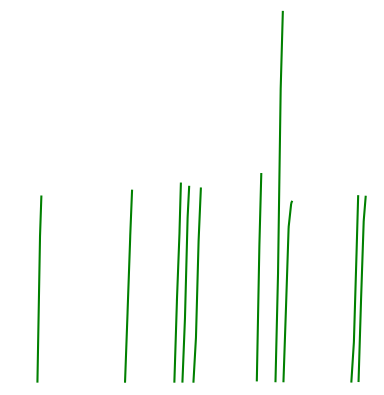

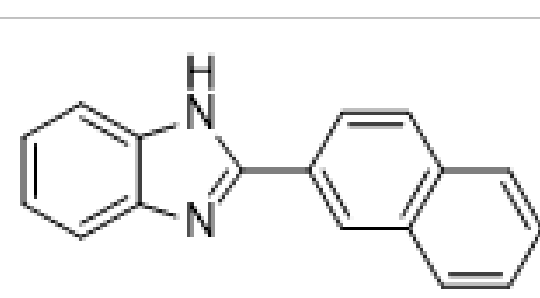

3h: ${ }^{1}$ H NMR 


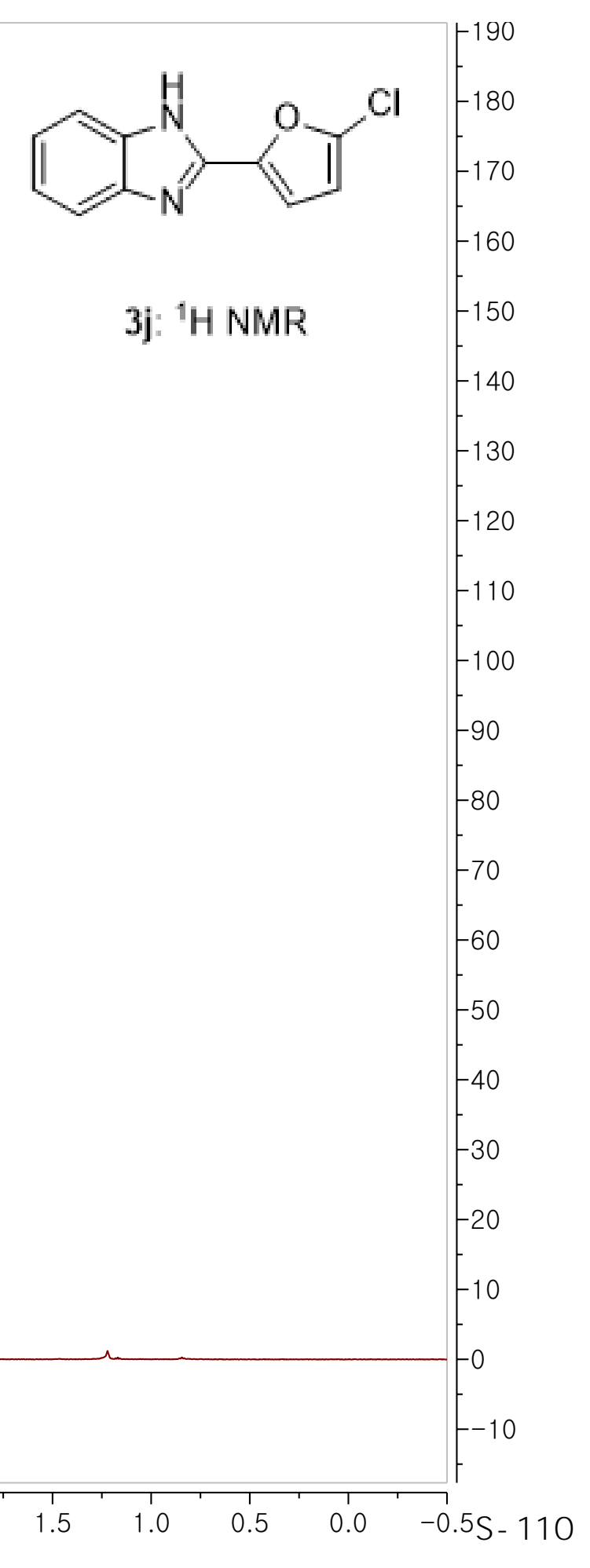




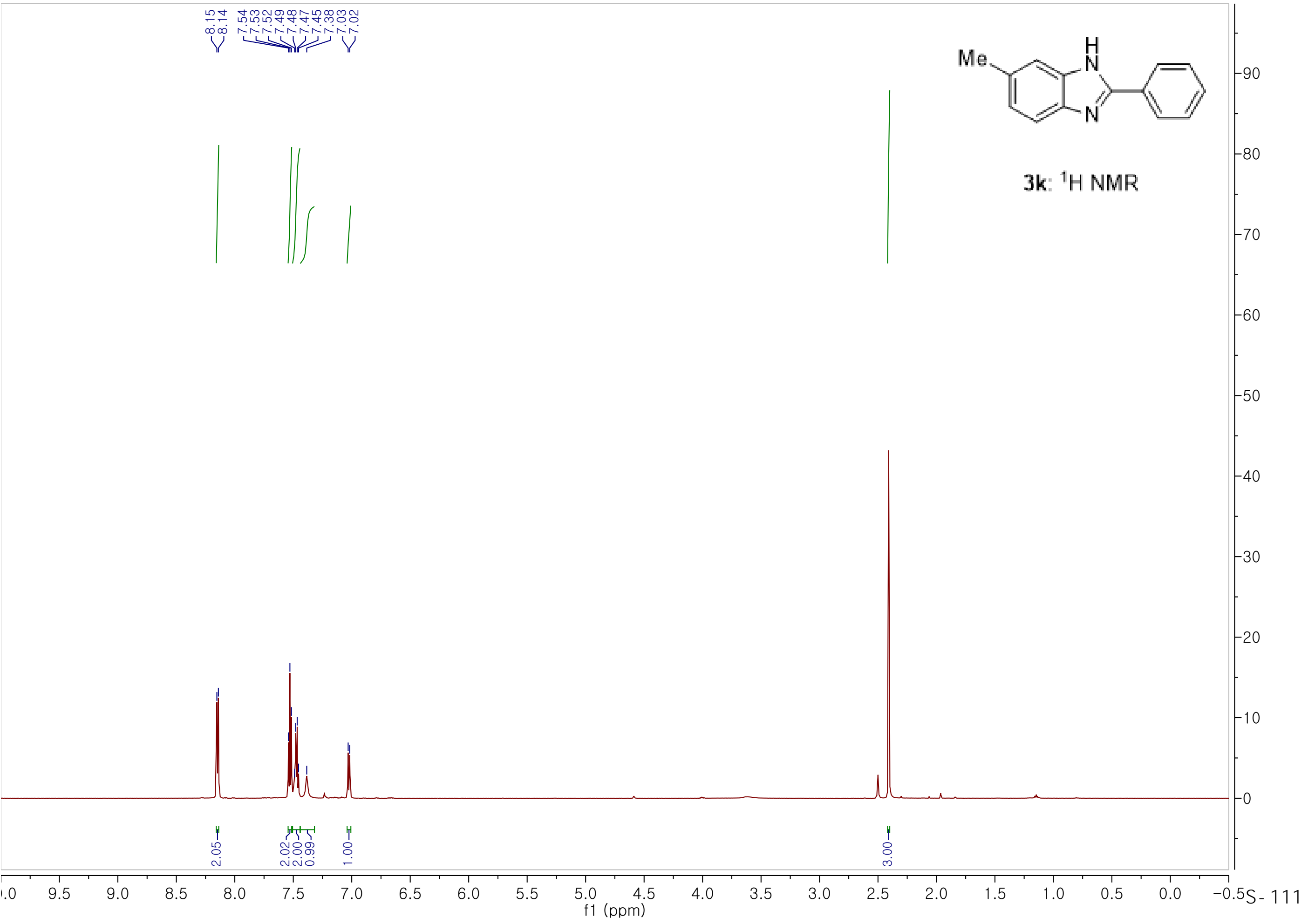


<smiles>Cc1ccc2nc(-c3ccccc3)[nH]c2c1</smiles>
3 k: ${ }^{13} \mathrm{C}$ NMR 

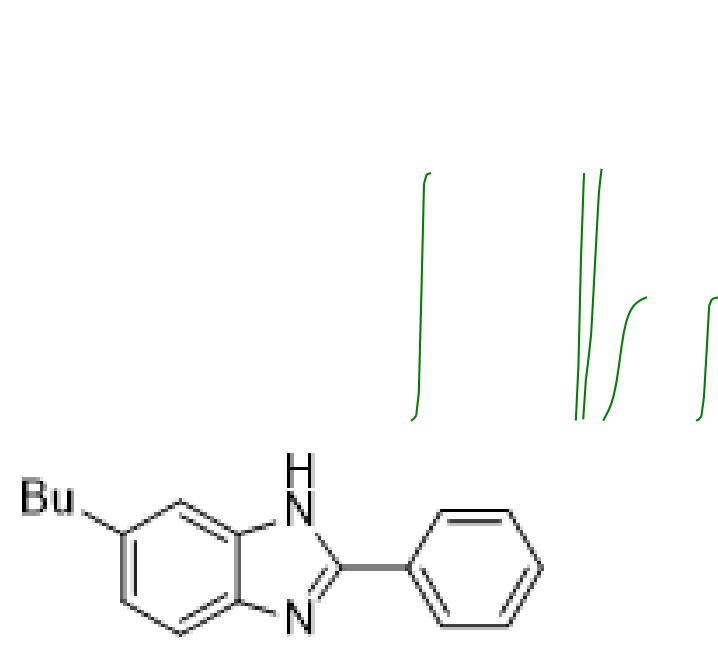

3I: ${ }^{1} \mathrm{H}$ NMR

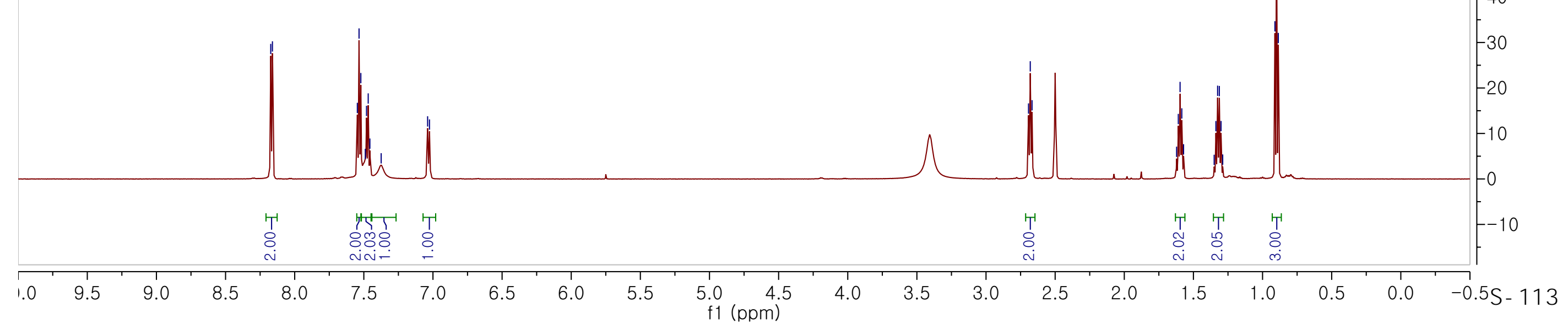



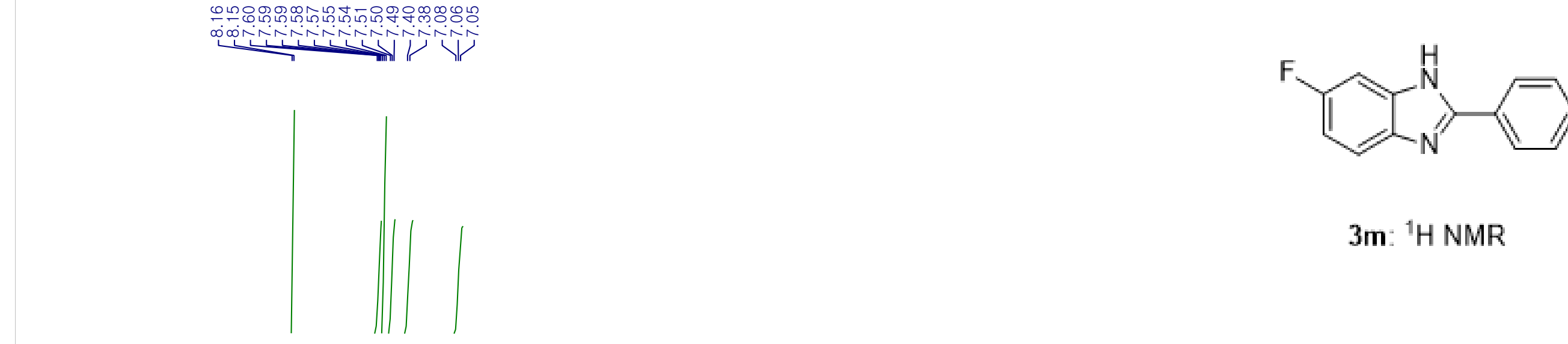

3m: ${ }^{1} \mathrm{H}$ NMR

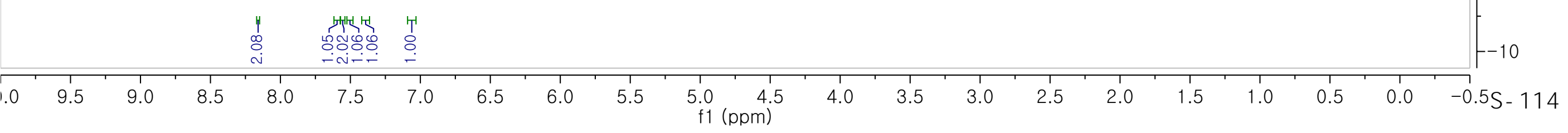




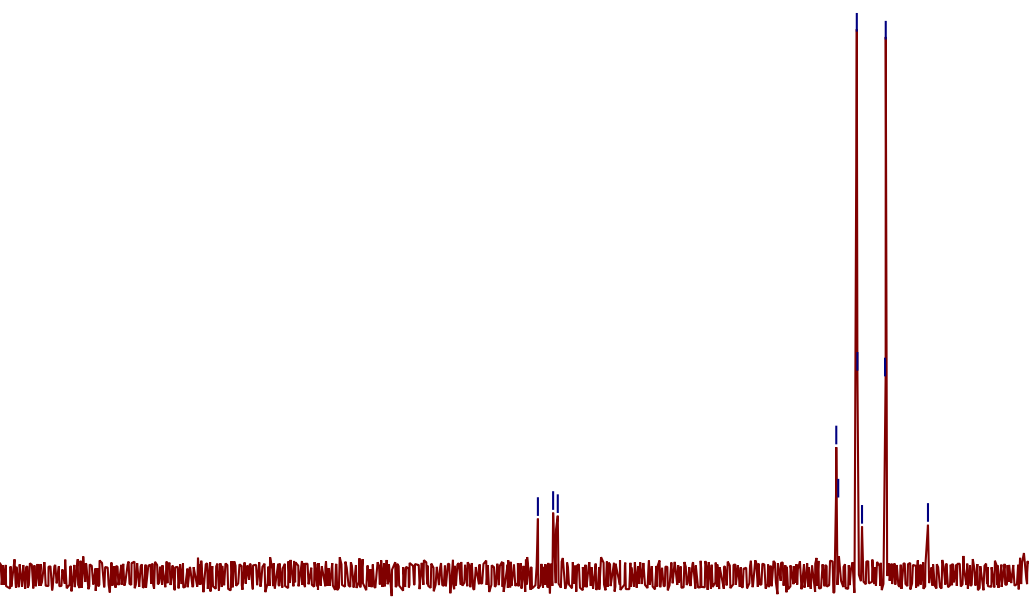

$3 n:{ }^{13} \mathrm{C}$ NMR<smiles>Fc1cc2nc(-c3ccccc3)[nH]c2cc1Cl</smiles> 

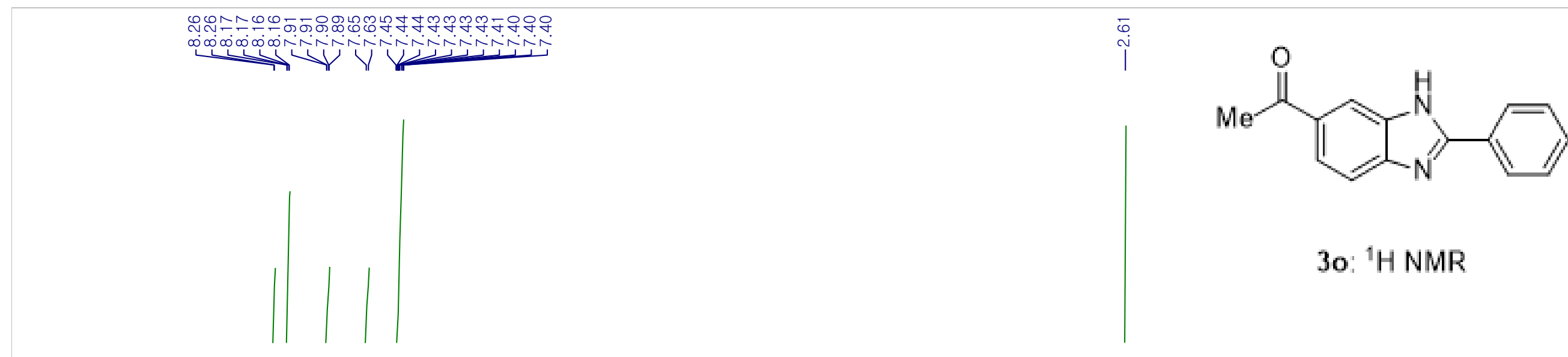

$-450$

$$
\mid
$$

3o: ${ }^{1}$ H NMR 

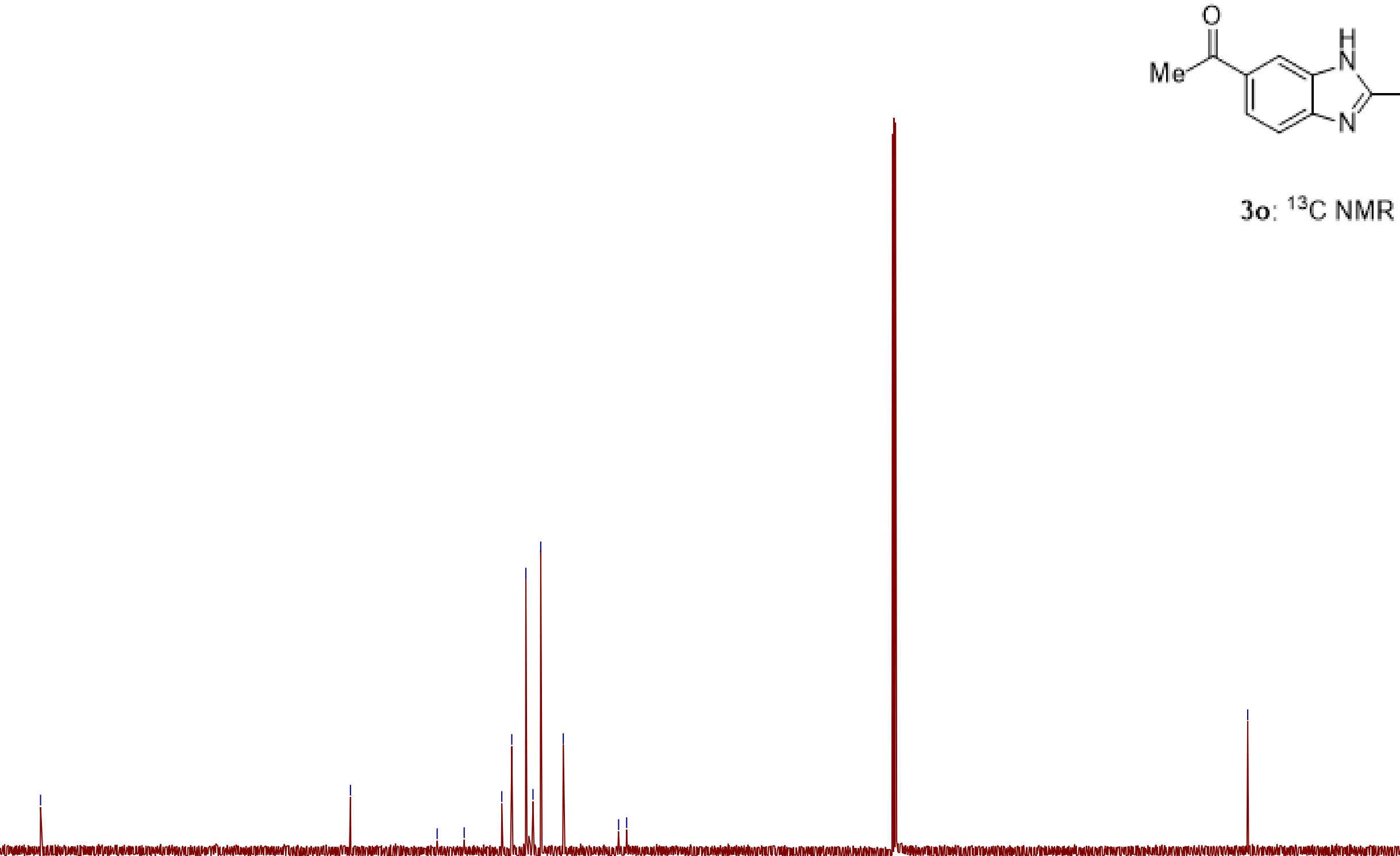<smiles>CC(=O)c1ccc2nc(-c3ccccc3)[nH]c2c1</smiles>

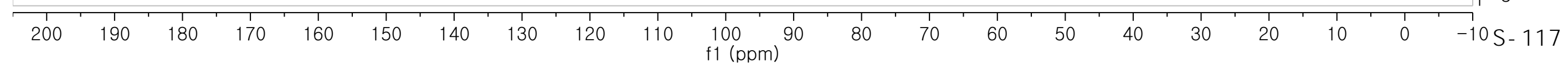




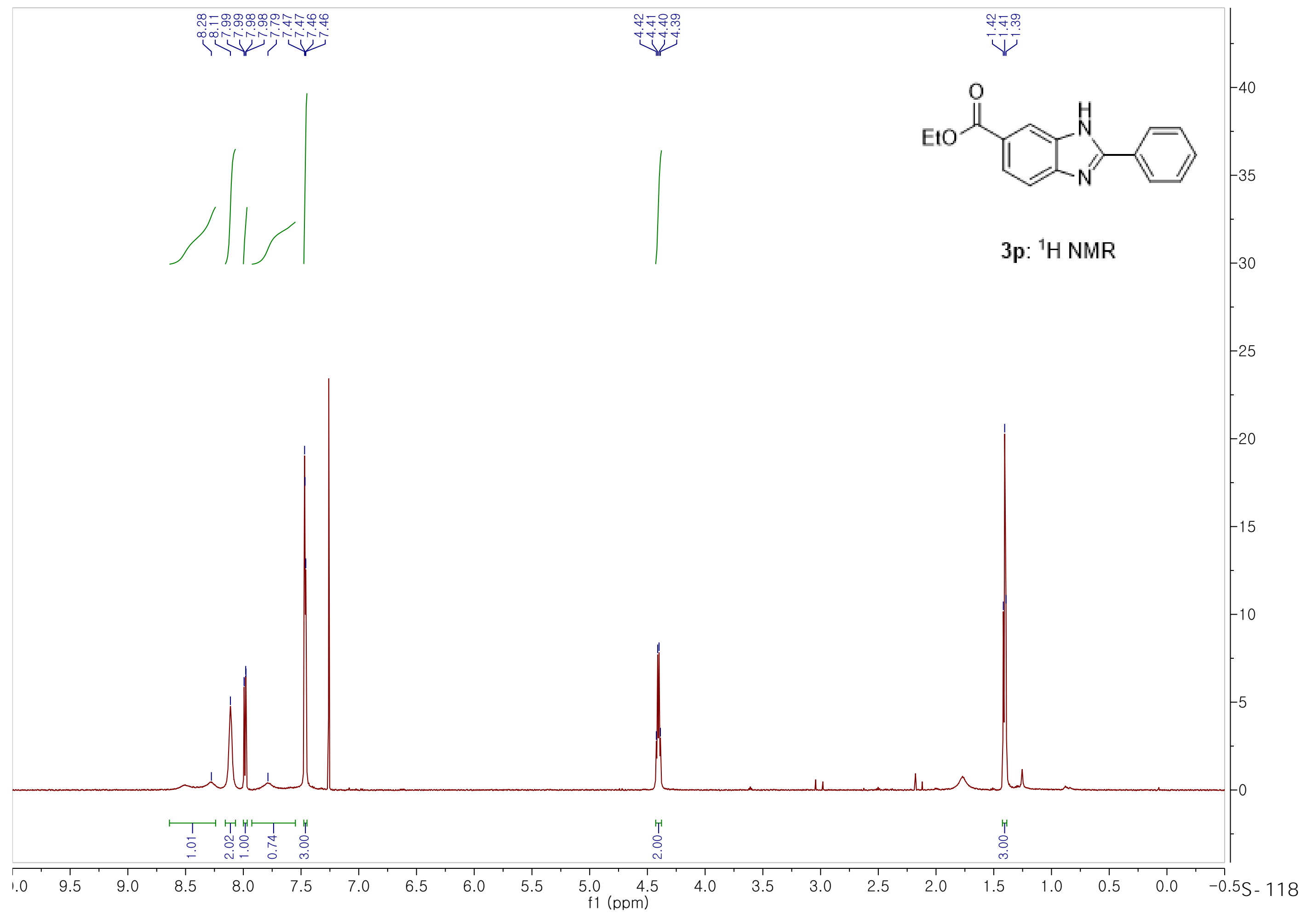




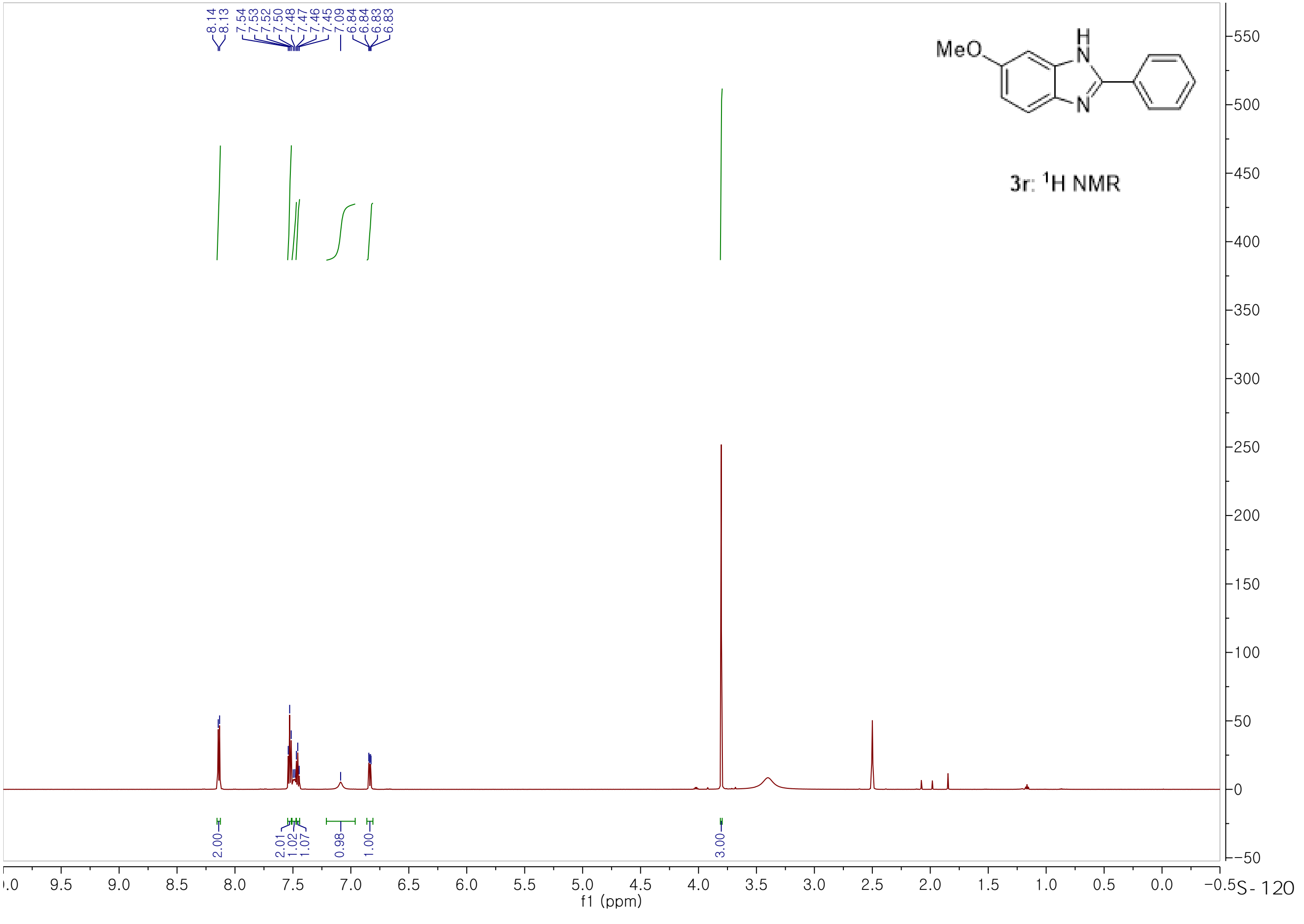


일

inivisio

$\stackrel{\substack{\infty \\ \sim \infty}}{\substack{\infty \\ i j}}$

$-100$
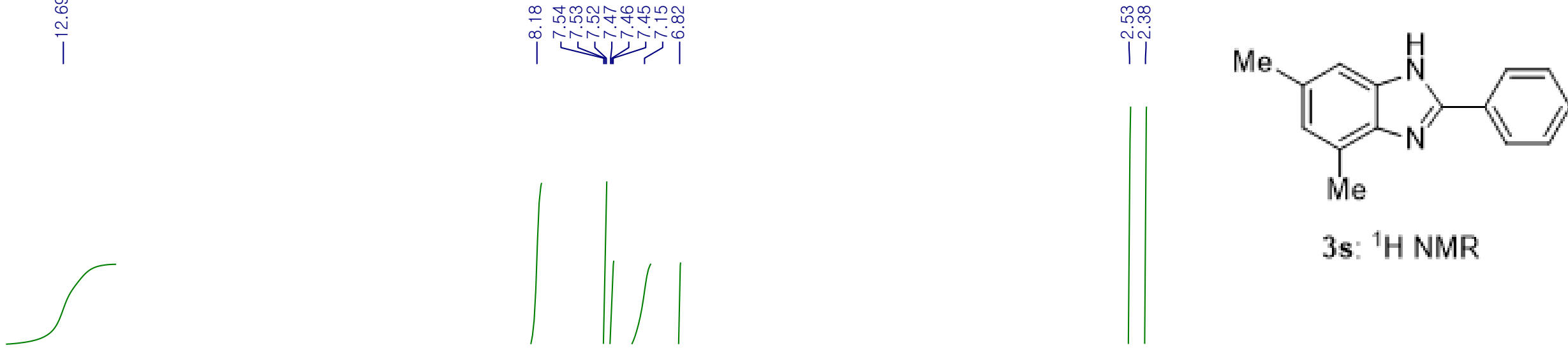

3s: ${ }^{1}$ H NMR 
<smiles>c1ccc(-c2nc3c(ccc4ccccc43)[nH]2)cc1</smiles>

3t: ${ }^{1} \mathrm{H}$ NMR 


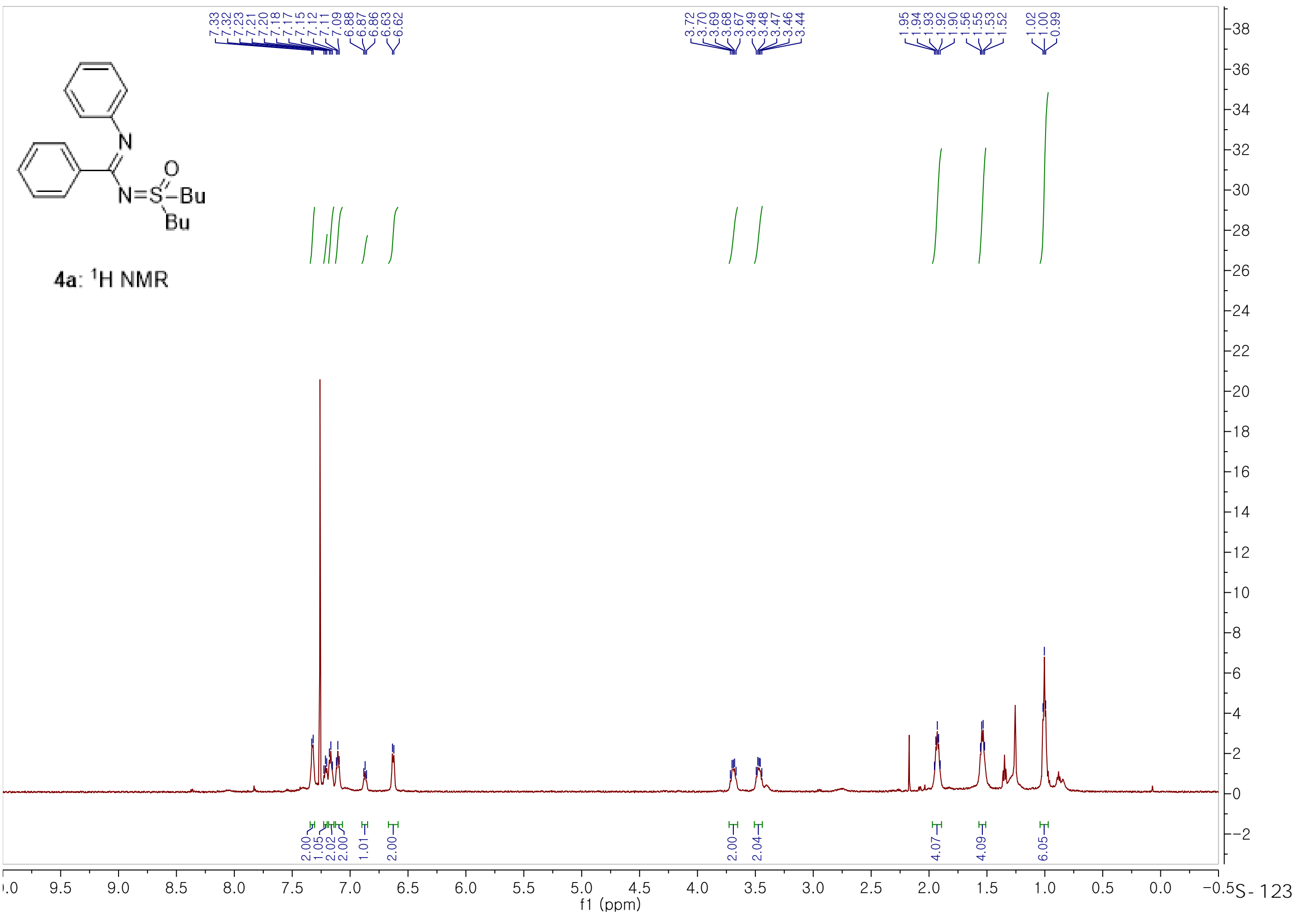




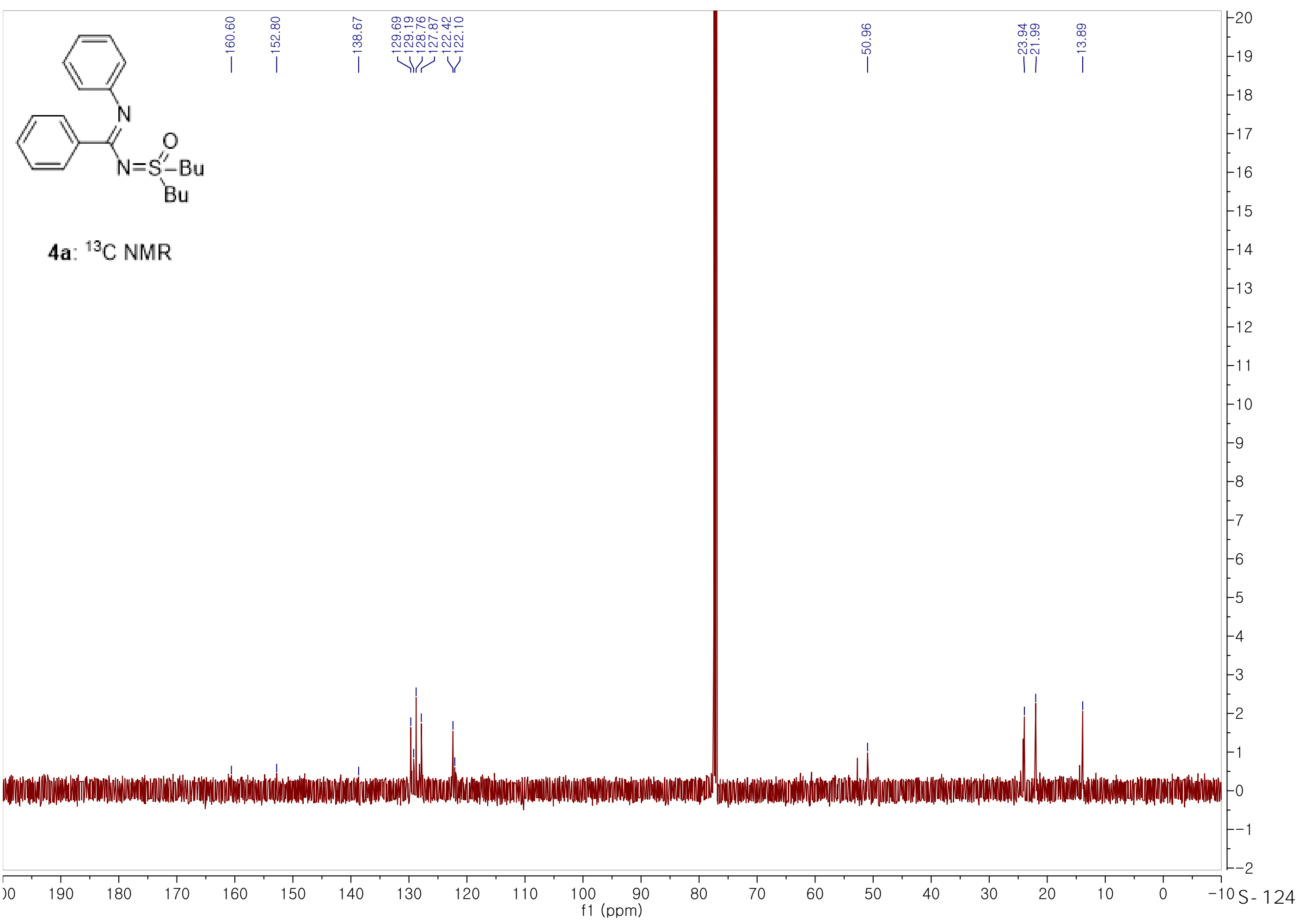




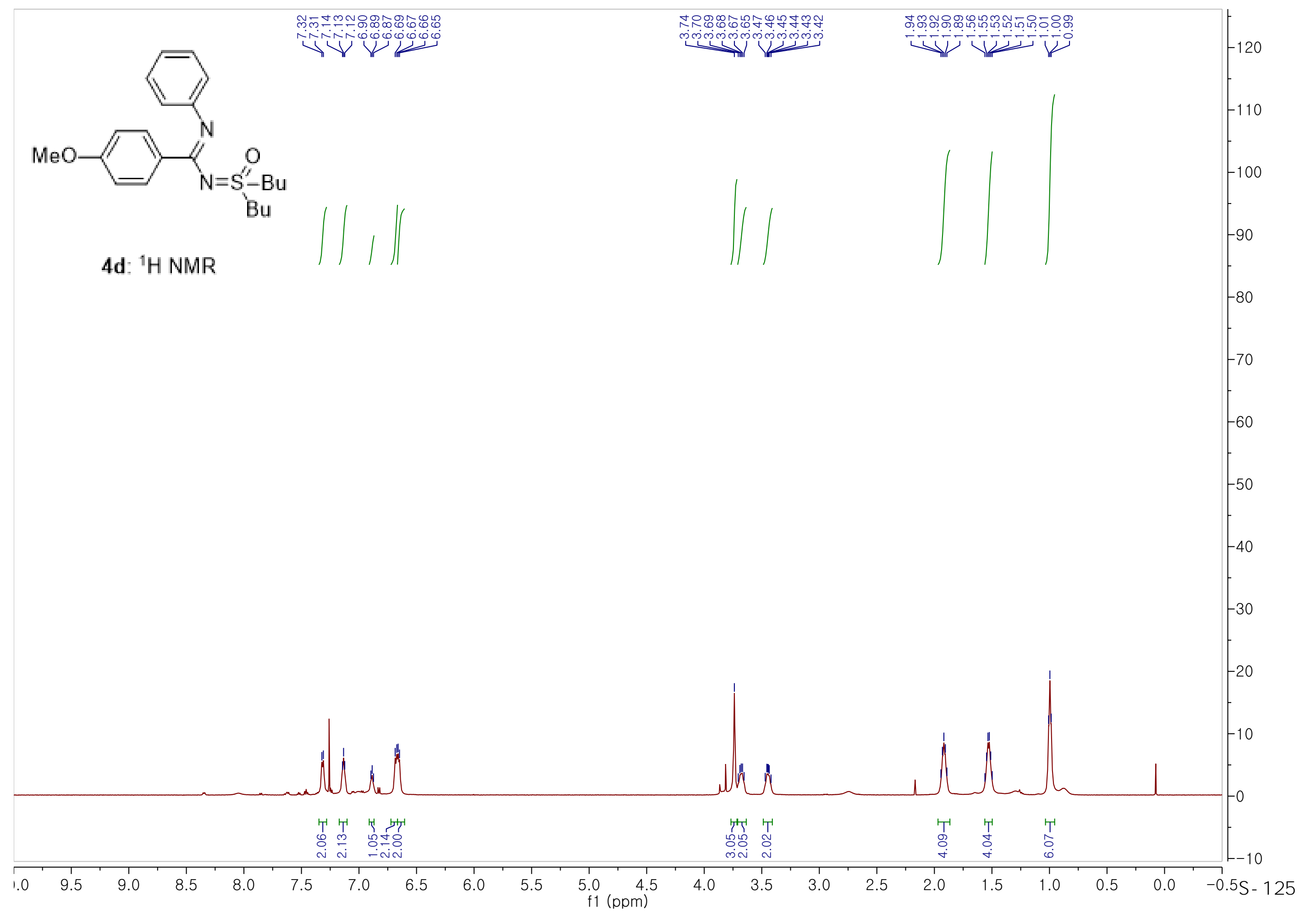




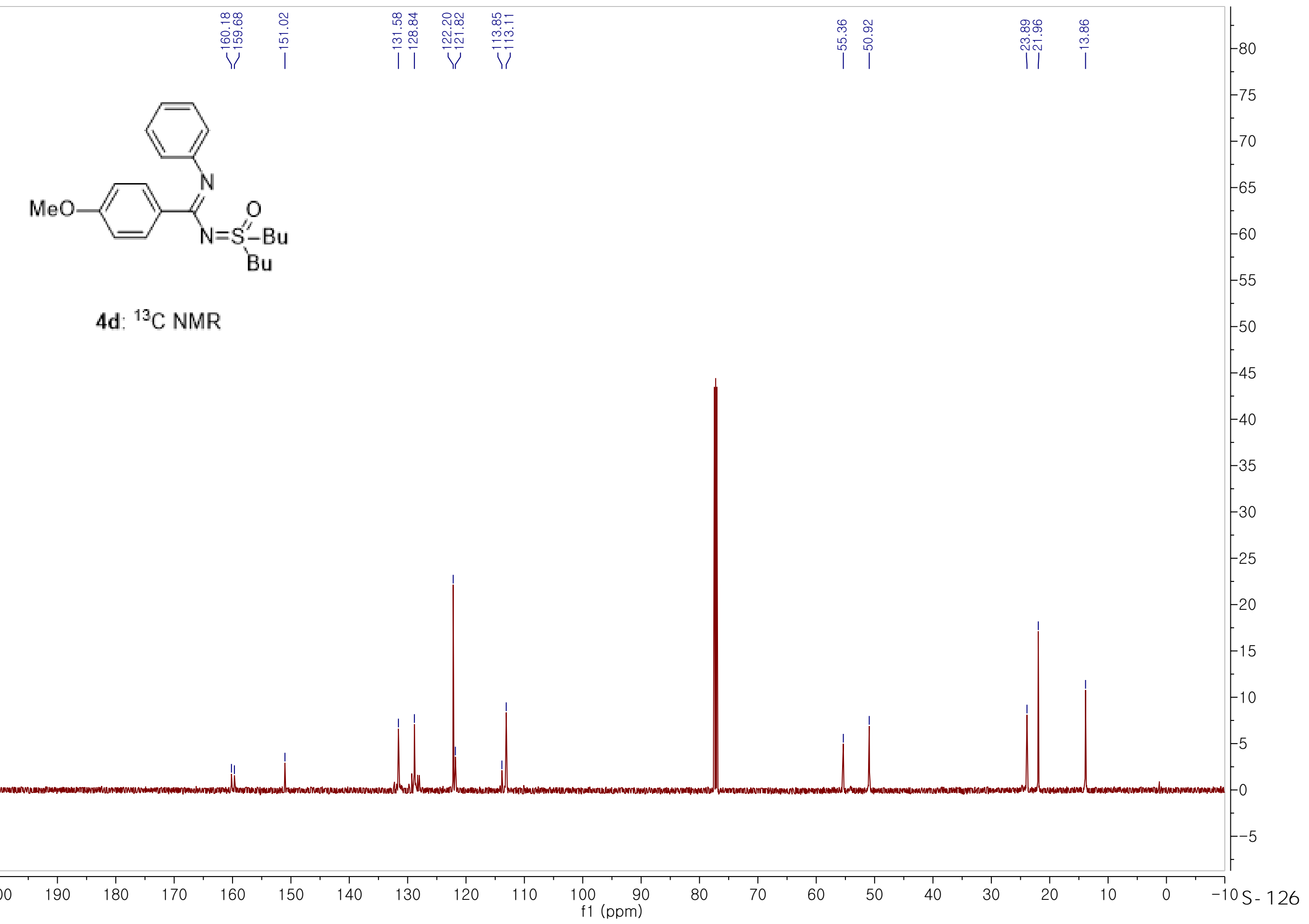




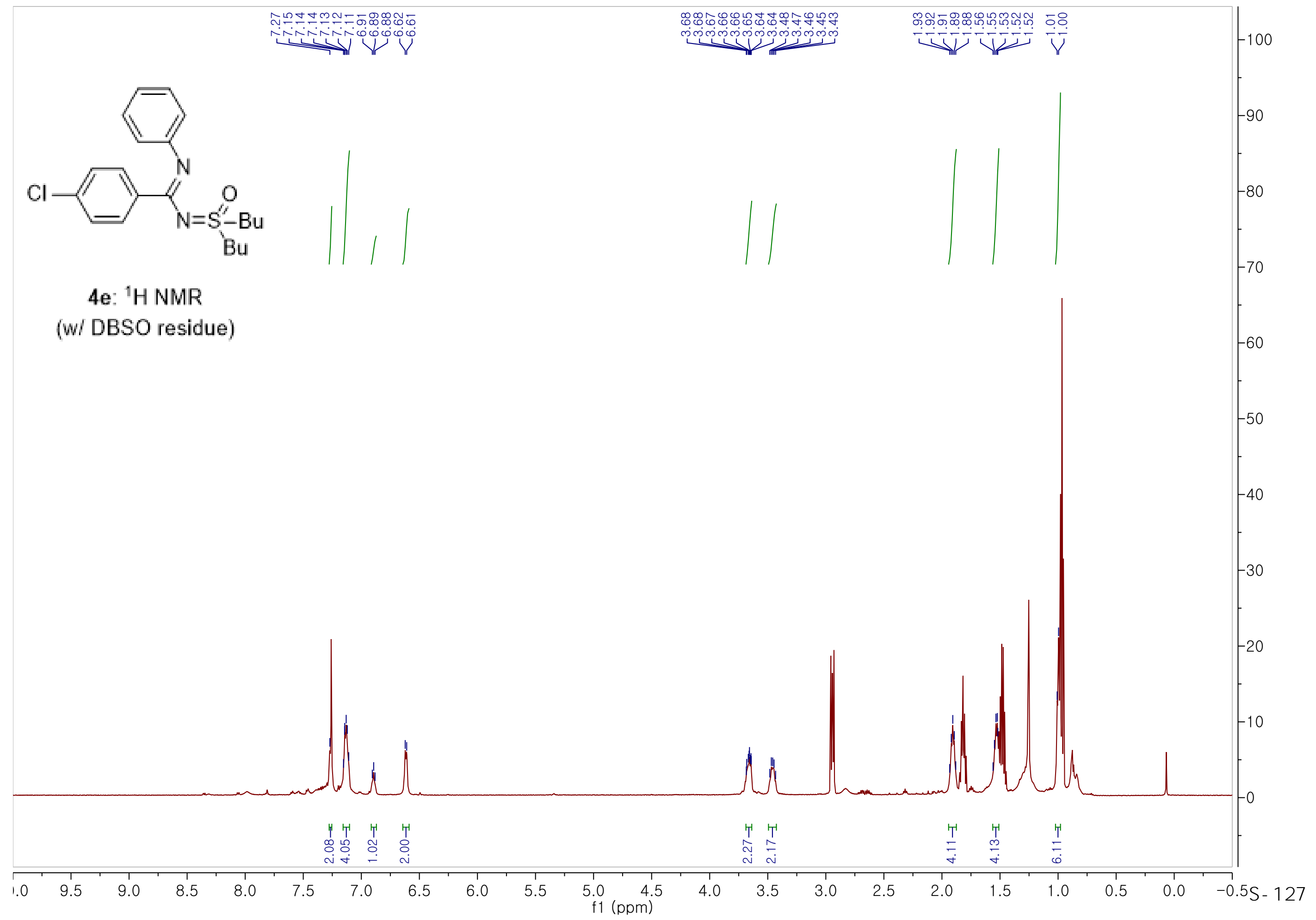




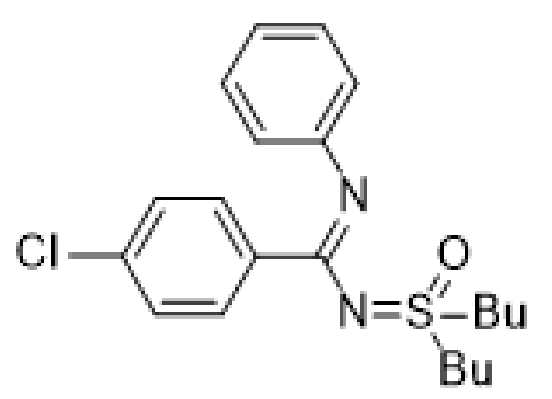

4e: ${ }^{13}$ C NMR

(w/ DBSO residue)

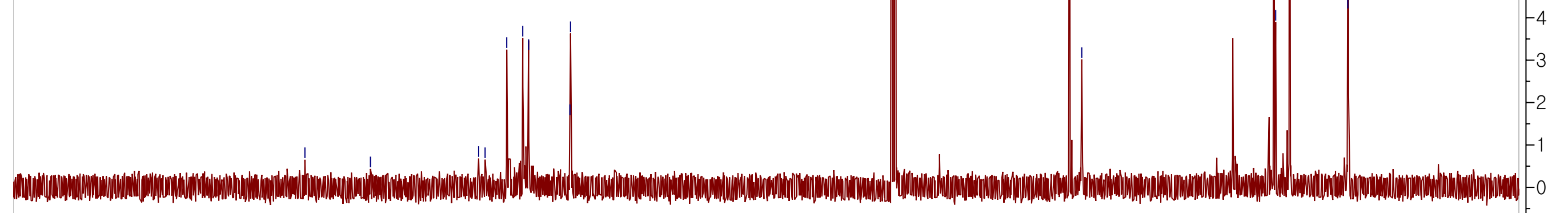




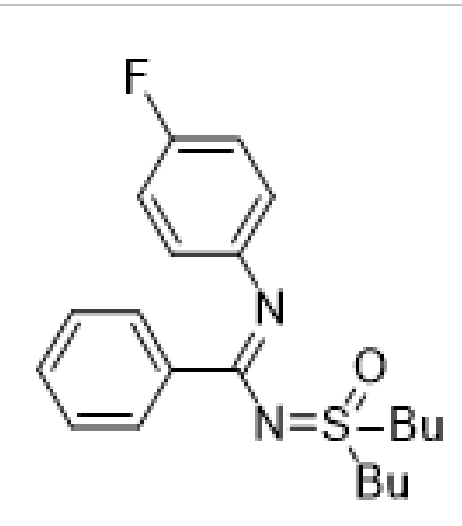

4m: ${ }^{1} \mathrm{H}$ NMR

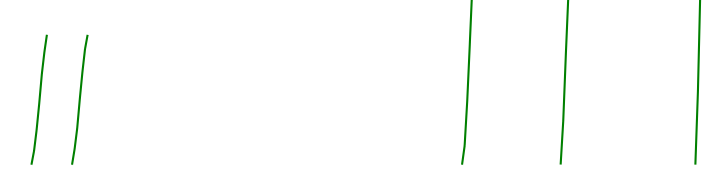




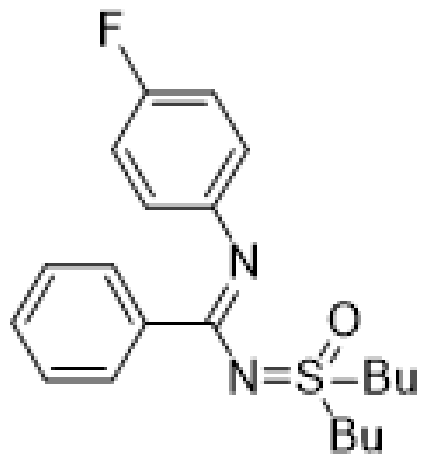

$4 \mathrm{~m}:{ }^{13} \mathrm{C}$ NMR 


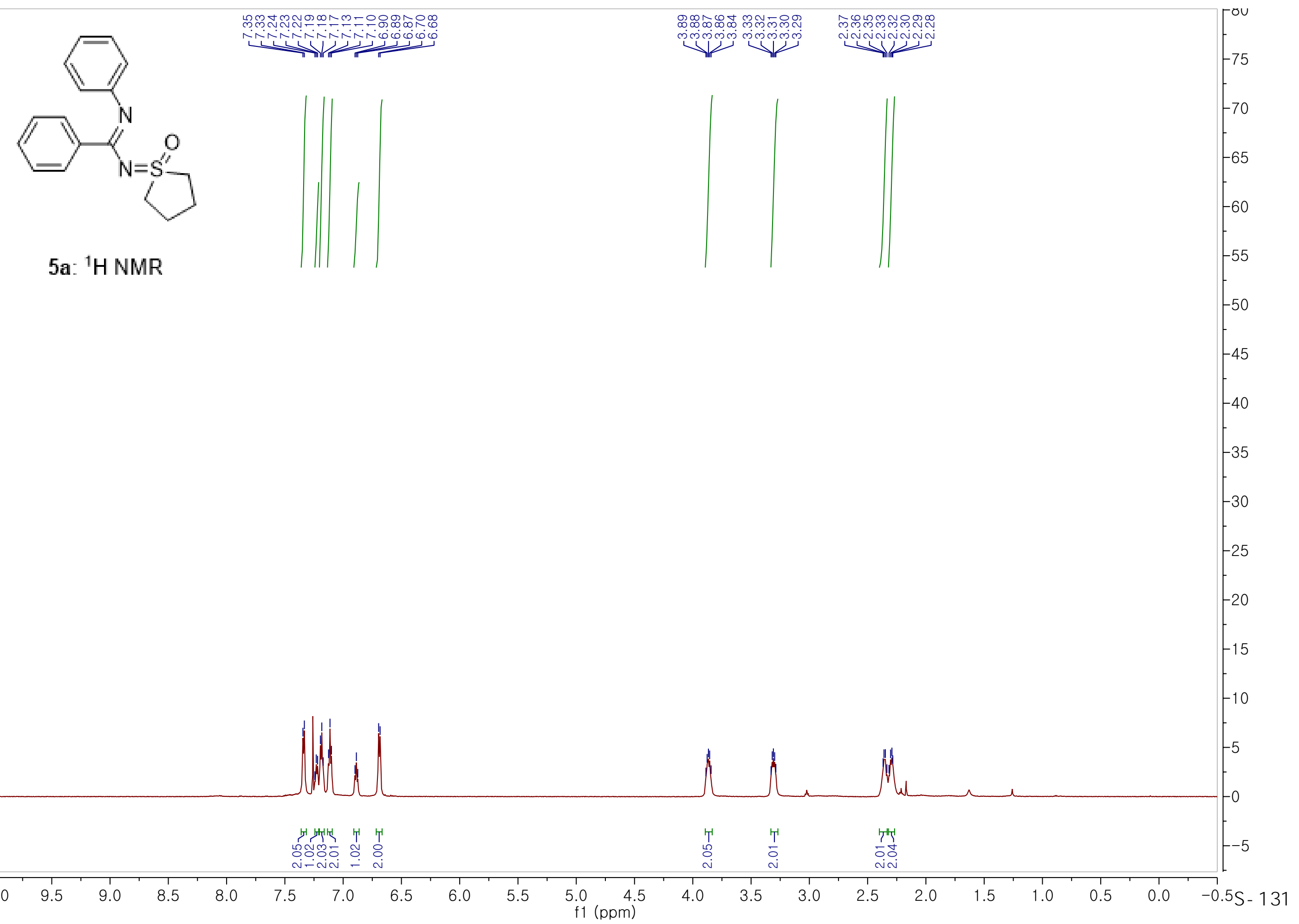



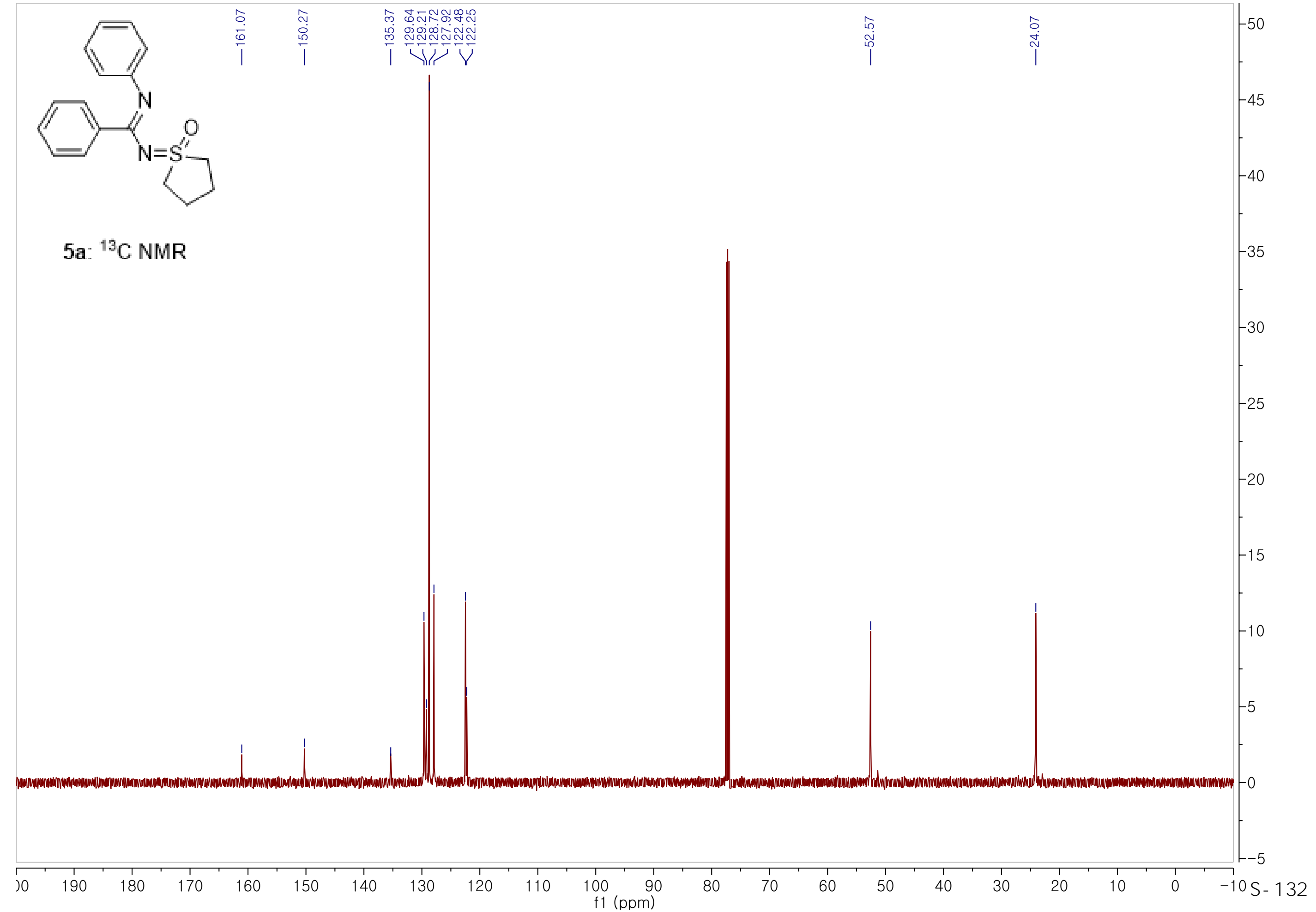


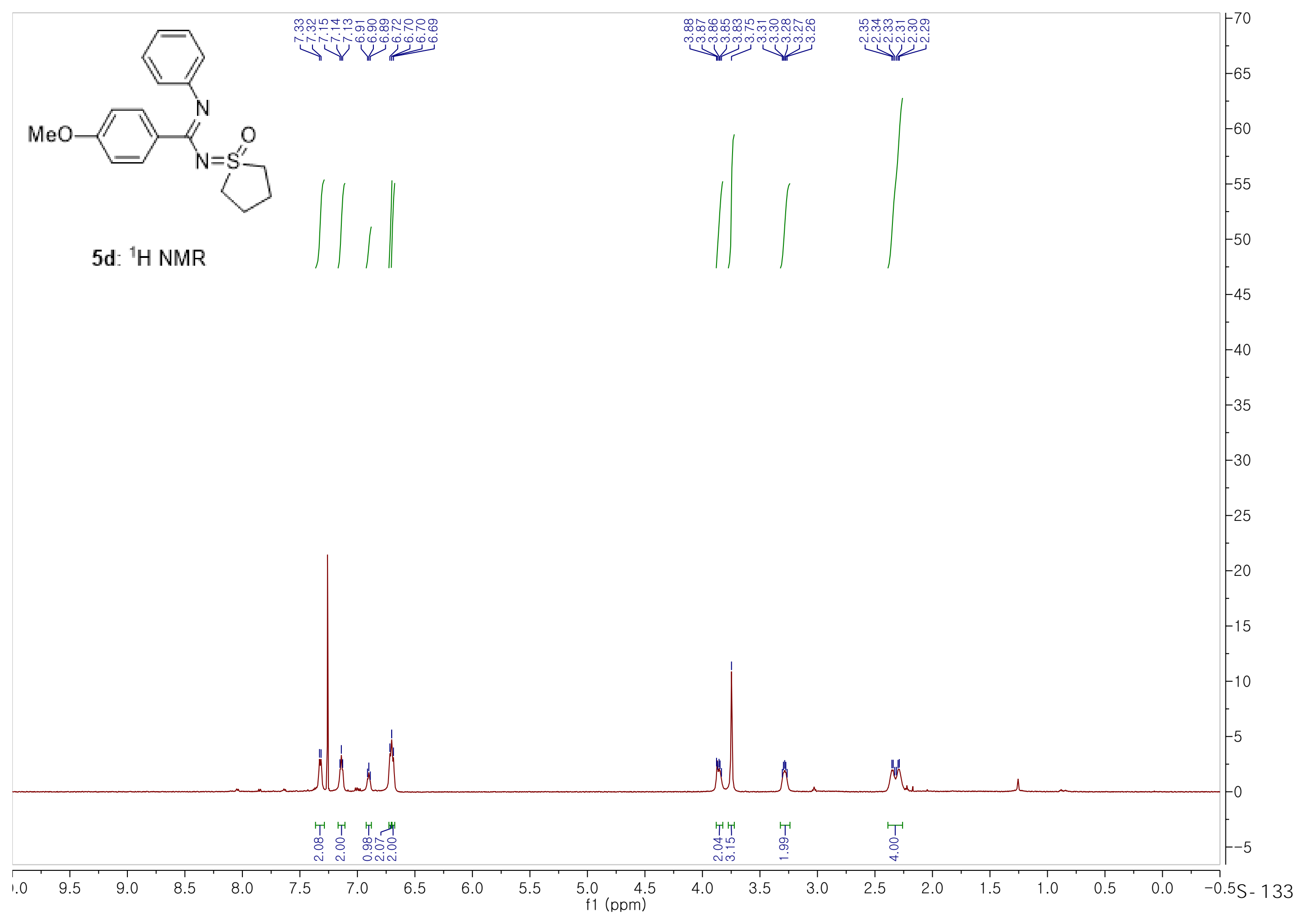



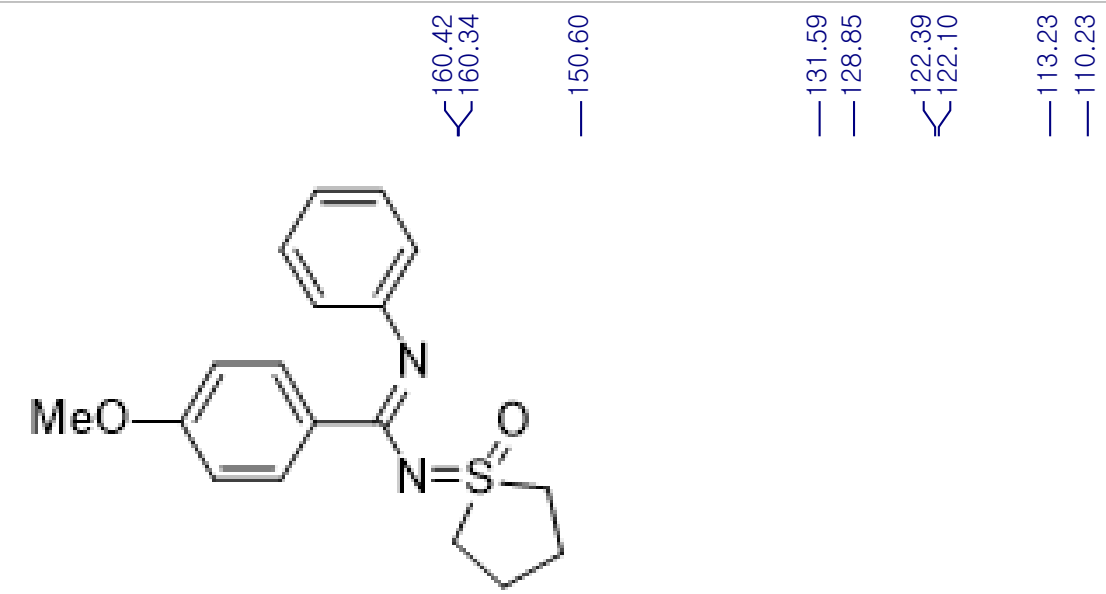

5d: ${ }^{13} \mathrm{C}$ NMR

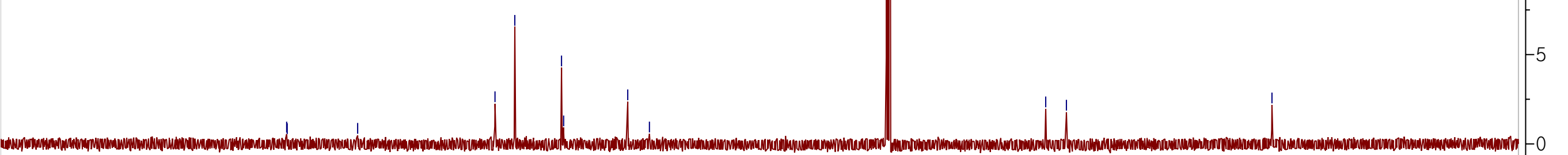

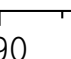

180

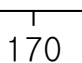

$10 \quad 160$

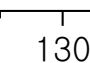

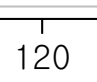

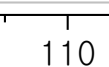




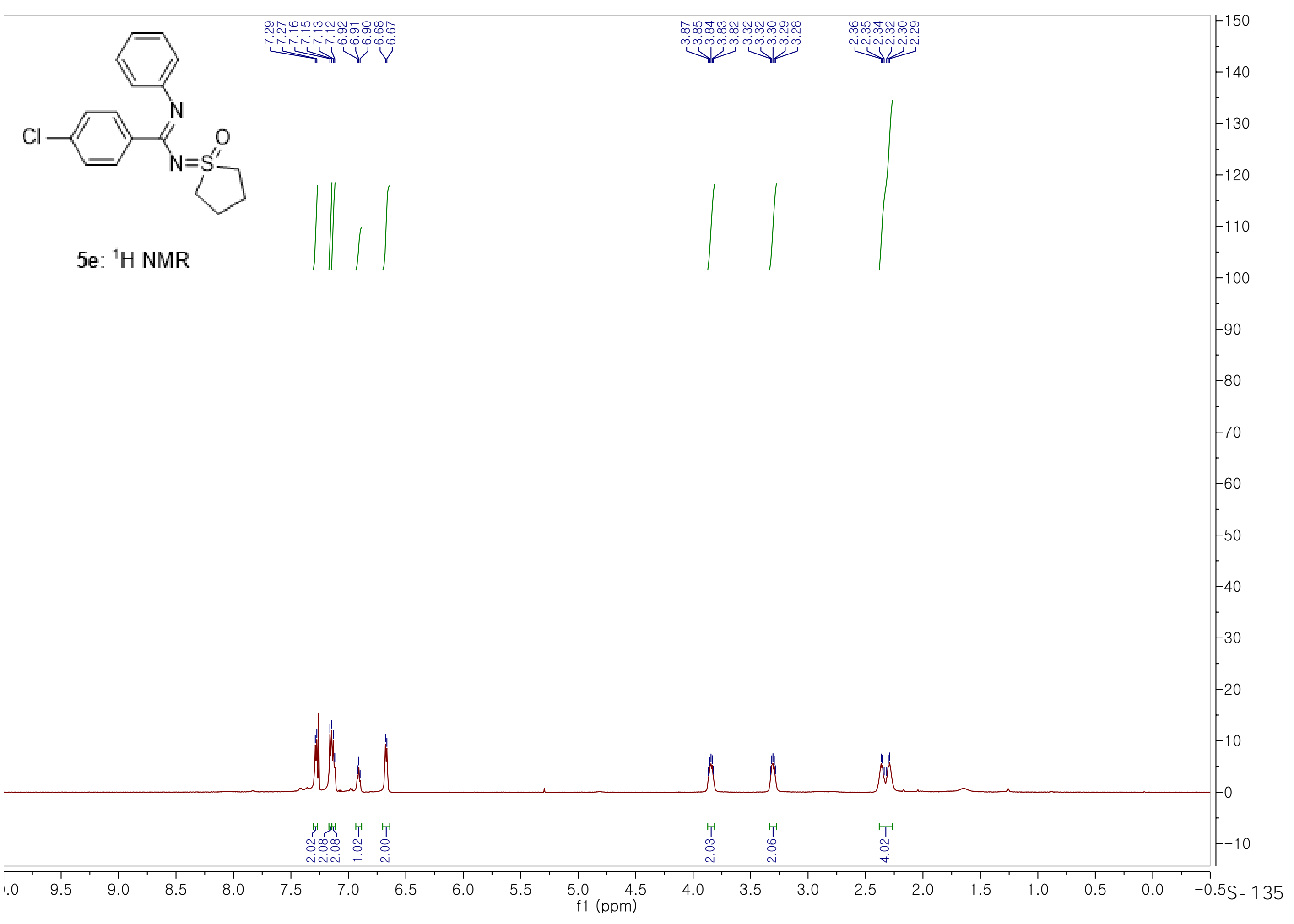




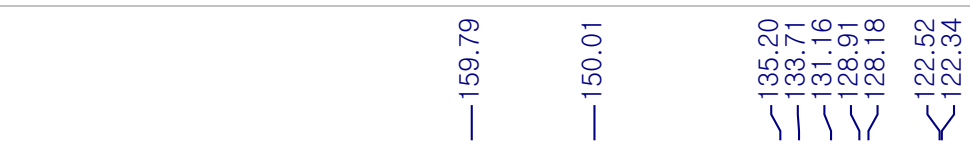

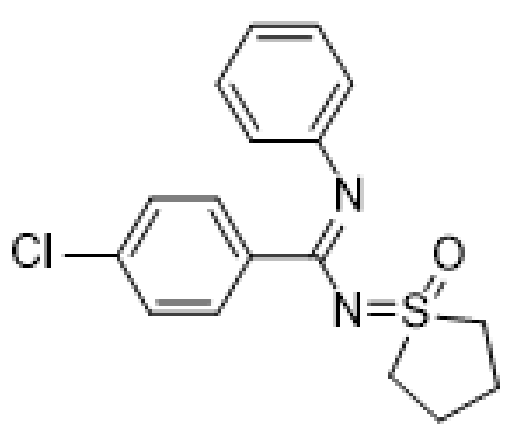

5e: ${ }^{13} \mathrm{C}$ NMR

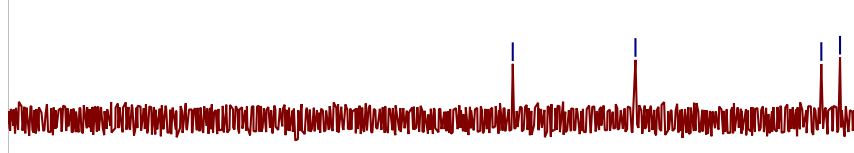

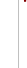




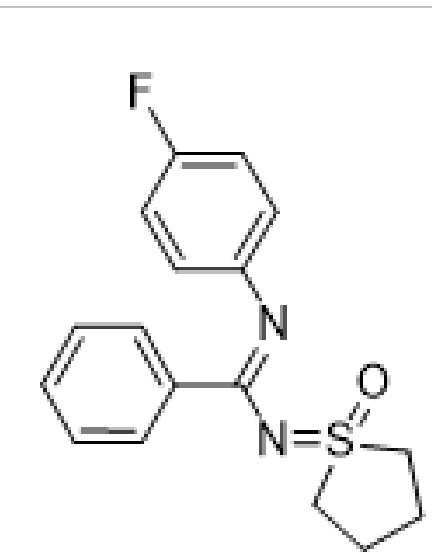

5m: ${ }^{1} \mathrm{H}$ NMR
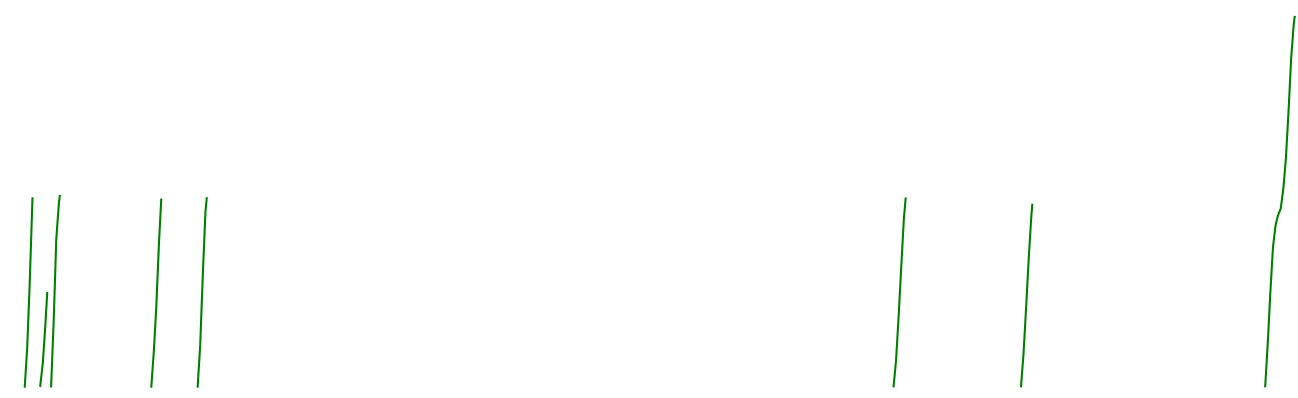


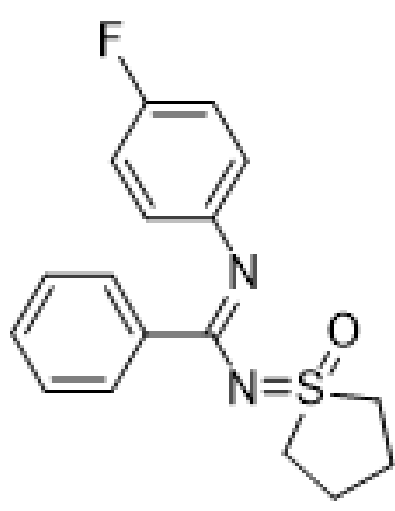

$5 m:{ }^{13}$ C NMR

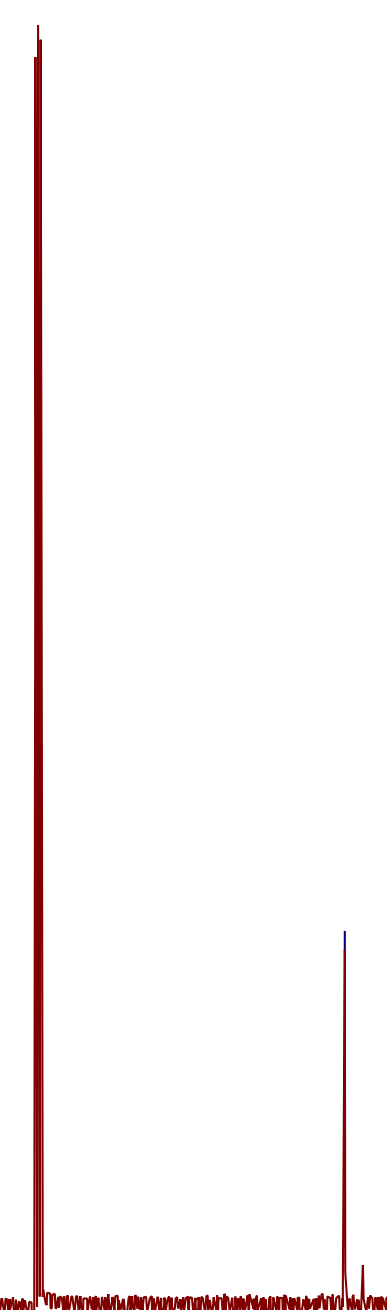

